\title{
Solvent-Dependent Mechanism and Stereochemistry of Mitsunobu Glycosylation with Unprotected Pyranoses
}

Hironori Takeuchi ${ }^{1}$, Yusuke Fujimori ${ }^{1}$, Yoshihiro Ueda ${ }^{1}$, Hiromitsu Shibayama ${ }^{1}$, Masaru Nagaishi ${ }^{1}$,

Tomoyuki Yoshimura $^{2}$, Takahiro Sasamori ${ }^{3}$, Norihiro Tokitoh ${ }^{1}$, Takumi Furuta ${ }^{4}$, Takeo Kawabata ${ }^{1^{*}}$

${ }^{1}$ Institute for Chemical Research, Kyoto University, Gokasho, Uji, Kyoto 611-0011, Japan. ${ }^{2}$ Division of Pharmaceutical Sciences, Graduate School of Medical Sciences, Kanazawa University, Kakuma-machi, Kanazawa 920-1192, Japan. ${ }^{3}$ Graduate School of Natural Sciences, Nagoya City University, Yamanohata 1, Mizuho-cho, Mizuho-ku, Nagoya, Aichi 467-8501, Japan. ${ }^{4}$ Department of Pharmaceutical Chemistry, Kyoto Pharmaceutical University, Shichonocho 1, Misasagi, Yamashina, Kyoto 607-8412, Japan.

\section{Table of Contents}

I. General Experimental Considerations $\quad$ S-2

II. Reagents $\quad$ S-2

III. Experimental Procedures and Characterization Data $\quad$ S-3

General Procedures (Scheme S1) $\quad$ S-3

Preparation and properties of powdered D-glucose (Figure S1) S-4

Polarization microscope image of commercial D-glucose (Figure S2) S-4

Anomeric ratio of several commercial D-glucose (Table S1) S-5

Optimization of the reaction conditions for $\beta$-selective glycosylation using $\alpha$-D-glucose (Table S2) $\quad$ S-5

Mechanistic insights using epimerized D-glucose (Scheme S2) S-6

$\begin{array}{ll}\text { Specific procedures and characterization data of the glycosides } & \text { S-7 }\end{array}$

$\begin{array}{ll}\text { IV. } & \text { KIE Measurements (Table S3) }\end{array}$

$\begin{array}{ll}\text { V. NMR Experiments (Figure S3) } & \text { S-26 }\end{array}$

VI. X-ray Cystal Structural Analysis of 2f $\quad$ S-30

$\begin{array}{lll}\text { VII. } & \text { References } & \text { S-38 }\end{array}$

VIII. $\quad{ }^{1}$ H-NMR and ${ }^{13}$ C-NMR Spectra Reprints $\quad$ S-39 


\section{GENERAL EXPERIMENTAL CONSIDERATIONS}

All reactions were carried out in an argon atmosphere under anhydrous conditions, and were stirred with Teflon-coated magnetic stir bars. Anhydrous acetonitrile $\left(\mathrm{CH}_{3} \mathrm{CN}\right)$, 1,4-dioxane, tetrahydrofuran (THF), $N, N$-dimethylformamide (DMF), methanol $(\mathrm{MeOH})$, chloroform $\left(\mathrm{CHCl}_{3}\right)$, dichloromethane $\left(\mathrm{CH}_{2} \mathrm{Cl}_{2}\right)$, isopropanol $(i-\mathrm{PrOH})$ were purchased from commercial suppliers and stored over activated molecular sieves. All other solvents were used as received unless otherwise noted. Yields were referred to isolated yield of analytically pure material unless otherwise noted. Reactions were magnetically stirred and monitored by thin-layer chromatography (TLC) using Silica gel 60 F254 precoated plates ( $0.25 \mathrm{~mm}$, Merck). Visualization was accomplished with UV light and $p$-anisaldehyde stain followed by heating. Purification of the reaction products was carried out by flash column chromatography using Ultra Pure Silica Gel (230-400 mesh) purchased from SILYCYCLE, unless otherwise noted. Infrared (IR) spectra were recorded using a JASCO FT-IR 4200 spectrometer and are reported in reciprocal centimeters $\left(\mathrm{cm}^{-1}\right) .{ }^{1} \mathrm{H}$ NMR spectra were recorded on JEOL ECX-400 (400 MHz), JEOL ECA-600 (600 MHz), Bruker Avance 800 (800 MHz), and are reported in ppm using solvent resonance as the internal standard (acetone- $d_{6}$ at $2.05 \mathrm{ppm}, \mathrm{CDCl}_{3}$ at $7.26 \mathrm{ppm}$, $\mathrm{CD}_{3} \mathrm{CN}$ at $1.94 \mathrm{ppm}, \mathrm{DMSO}-d_{6}$ at $2.49 \mathrm{ppm}, \mathrm{MeOH}-d_{4}$ at $\left.3.31 \mathrm{ppm}\right) .{ }^{1} \mathrm{H}$ NMR data are reported as follows: chemical shift; multiplicity; coupling constants $(\mathrm{Hz})$; number of hydrogen. Multiplicity is abbreviated as follows: $\mathrm{s}=$ singlet, $\mathrm{d}=$ doublet, $\mathrm{t}=$ triplet, $\mathrm{q}=$ quartet, quint $=$ quintet, $\mathrm{sext}=$ sextet, $\mathrm{dd}=$ double doublet, $\mathrm{ddt}$ $=$ double double triplet, $\mathrm{m}=$ multiplet, $\mathrm{br}=$ broad. Proton-decoupled ${ }^{13} \mathrm{C}$ NMR spectra were recorded on ECX-400 (100 MHz), ECA-600 (150 MHz), Bruker Avance 800 (200 MHz), and are reported in ppm using solvent resonance as the internal standard (acetone- $d_{6}$ at $29.84 \mathrm{ppm}, \mathrm{CDCl}_{3}$ at $77.16 \mathrm{ppm}, \mathrm{CD}_{3} \mathrm{CN}$ at 118.26 ppm, DMSO- $d_{6}$ at $39.52 \mathrm{ppm}, \mathrm{MeOH}-d_{4}$ at $49.00 \mathrm{ppm}$ ). High-resolution mass spectra (HRMS) were obtained using JEOL-DX 700 mass spectrometer for FAB (TOF) and Impact HD (Bruker Daltonics) for ESI (TOF). Specific rotations were measured with JASCO P-2200 digital polarimeter using the sodium D line and are reported as follows: $[\alpha]_{D}{ }^{t}(c=10 \mathrm{mg} / \mathrm{mL}$, solvent). Melting points were measured with Micro Melting Point Apparatus PM-500 (Yanagimoto) and are reported in degree Celsius $\left({ }^{\circ} \mathrm{C}\right)$.

\section{REAGENTS}

$\alpha$-D-Glucose was purchased from Becton Dickinson Inc. or Nacalai tesque Inc. and used after grinding (see: Preparation and properties of powdered D-glucose in Figure S1). $\beta$-D-Glucose, D-xylose, and D-mannose were purchased from TCI and used after grinding. D-Galactose was purchased from Kanto Chemical Co. Inc. and used after grinding. 3,4,5-Tris(benzyloxy)benzoic acid, ${ }^{1}$ 2-allyl-4-nitrophenol, ${ }^{2}$ 3-bromo-4-hydroxybenzaldehyde, ${ }^{3}$ and $\mathrm{NsNHBoc}^{4}$ were prepared according to literature procedures. All other reagents were purchased from commercial sources and used without further purification. 


\section{EXPERIMENTAL PROCEDURES AND CHARACTERIZATION DATA}

\section{General procedures for $\beta$-selective glycosylation (Scheme S1)}

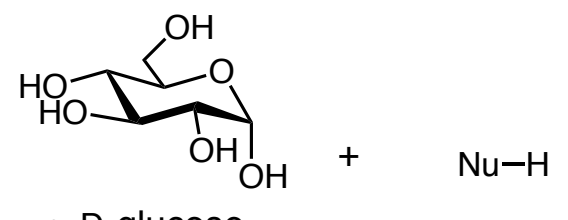

$\alpha$-D-glucose

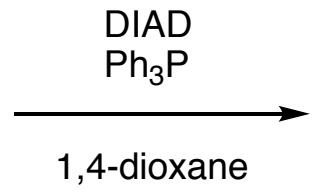

\section{General procedure A}

A round-bottom flask was charged with powdered $\alpha$-D-glucose (3.0 equiv.) and dehydrated 1,4-dioxane ( $0.03 \mathrm{M}$ of $\alpha$-D-glucose) under an Ar atmosphere (Note 1). After ultrasound irradiation of the suspension of $\alpha$-D-glucose in 1,4-dioxane for $15 \mathrm{~min}$, glycosyl acceptor $(\mathrm{NuH})$ (1.0 equiv.) and $\mathrm{Ph}_{3} \mathrm{P}$ (2.0 equiv.) were added. DIAD (2.0 equiv.) was added dropwise by syringe and the resulting mixture was stirred vigorously at rt for $30 \mathrm{~min}$ (Note 2). The reaction mixture was quenched with $\mathrm{MeOH}$, stirred for $5 \mathrm{~min}$, and concentrated in vacuo at $40{ }^{\circ} \mathrm{C}$ (water bath) to give a residue. The residue was directly purified by flash column chromatography $\left(\mathrm{SiO}_{2}, \mathrm{CHCl}_{3} / \mathrm{MeOH} 50: 1\right.$ to 5:1 v/v) to give the corresponding $\beta$-glycoside (Note 3).

\section{Notes}

1. Reagent-grade D-glucose from Becton Dickinson Inc. was used after grinding procedures (See S-4).

2. DIAD should be added dropwise by syringe (ca. 1 drop / $3 \mathrm{sec}$ ) after the orange color of each DIAD drop disappeared in the reaction mixture to avoid possible side reactions.

3. The product is a highly polar compound so that the extraction procedures should be avoided to prevent product loss. 


\section{Preparation and properties of powdered D-glucose (Figure S1)}

(a) Particle sizes of commercial D-glucose from Becton Dickinson Inc. (Difco ${ }^{\mathrm{TM}}$ Dextrose) were around 1 $\mathrm{mm}$. (b) About $5 \mathrm{~g}$ of commercial D-glucose was grinded with an $8 \mathrm{~cm}$-diameter mortar in open-air for 5 min. (c) A suspension of the powdered glucose in 1,4-dioxane was irradiated with ultrasound by an ordinary laboratory cleaner for 10 min prior to the reaction. (d) Powders of grinded glucose was not uniform, whose particle sizes were $<0.1 \mathrm{~mm}$.

(a)

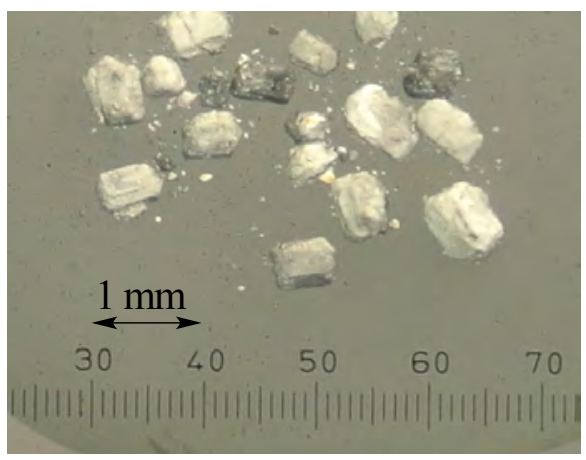

(c)

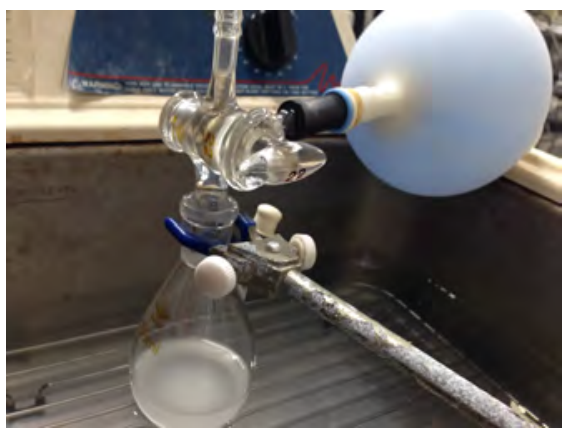

(b)

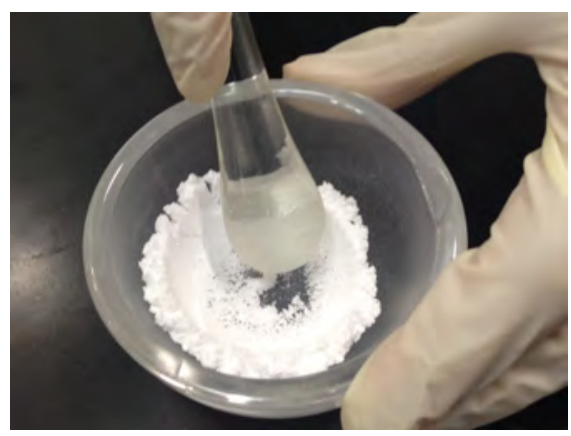

(d)

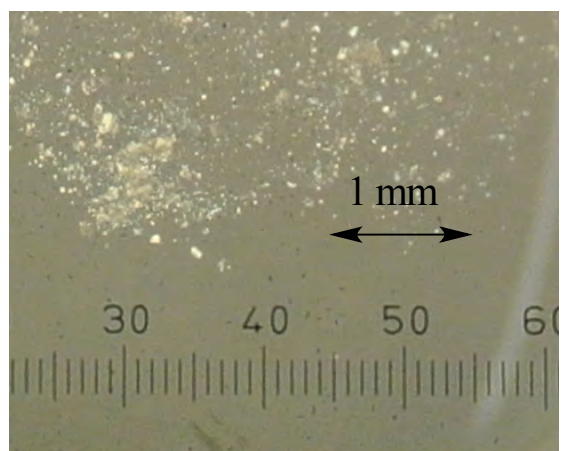

\section{Polarization microscope image of commercial D-glucose (Figure S2)}

We noticed that D-glucose we used was identified as single crystals by polarization microscope (OLYMPUS SZ60 microscope with the polarizer SZ-PO) as shown below, while we measured the particle size of commercial D-glucose. Interestingly, this single crystal consisted of $\alpha$-anomer of D-glucose. (See S-5) (a) Microscope image of D-glucose. (b) Polarization microscope image of D-glucose.

(a)

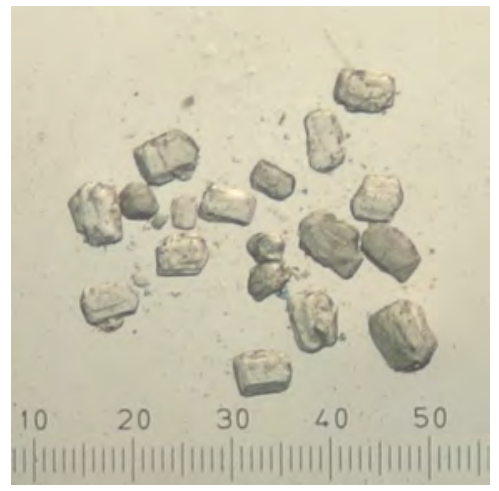

(b)

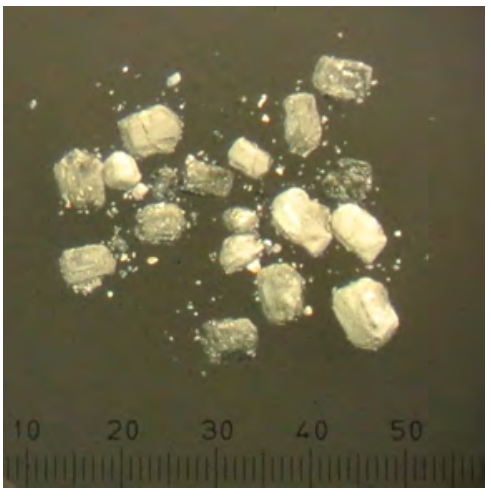




\section{Anomeric ratios of several commercially available D-glucose (Table S1)}

The anomeric ratio of D-glucose was determined by ${ }^{1} \mathrm{H}-\mathrm{NMR}$ in DMSO- $d_{6}(\mathrm{ca} .0 .6 \mathrm{~mL})+2$ drops of $\mathrm{D}_{2} \mathrm{O}$ at $293 \mathrm{~K}$ immediately after D-glucose was dissolved. While D-glucose epimerizes easily in protic polar solvent, the epimerization of anomeric hydroxyl group of D-glucose in aprotic solvent is known to be slow. ${ }^{5}$

\begin{tabular}{|l|l|l|l|}
\hline Reagent & Supplier & Cat. No. & $\alpha / \beta$ ratio \\
\hline Difco ${ }^{\mathrm{TM}}$ Dextrose & Becton Dickinson & 215530 & $\alpha / \beta=100 / 0$ \\
\hline D-(+)-Glucose, Anhydrous & Nacalai tesque & $168-06$ & $\alpha / \beta=100 / 0$ \\
\hline D-(+)-Glucose & KANTO CHEMICAL & $10017-30$ & $\alpha / \beta=99 / 1$ \\
\hline D-(+)-Glucose, ACS reagent & SIGMA-ALDRICH & G5767-25G & $\alpha / \beta=96 / 4$ \\
\hline
\end{tabular}

\section{Optimization of the reaction conditions for $\beta$-selective glycosylation using $\alpha$-D-glucose (Table S2)}

Procedure: A round-bottom flask was charged with $\alpha$-D-glucose and solvent ( $0.03 \mathrm{M}$ of $\alpha$-D-glucose). Benzoic acid (1.0 equiv.) and $\mathrm{Ph}_{3} \mathrm{P}$ (2.0 equiv.) were added. DIAD (2.0 equiv.) was slowly added by syringe causing the orange color to immediately disappear in every drops and the resulting mixture was stirred vigorously. The reaction mixture was quenched with $\mathrm{MeOH}$. Major byproducts in this glycosylation reaction were 6-O-acyl- $\beta$-D-glucoside and benzoic anhydride.

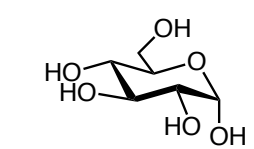

$\alpha$-D-glucose

\begin{tabular}{|c|c|c|c|c|c|c|c|}
\hline entry & solvent & $\mathbf{E}_{\mathbf{T}} \mathbf{N} a$ & time (min) & Glc (eq.) & $\begin{array}{c}\beta \text {-glycoside } \\
(\%)^{b}\end{array}$ & $\alpha / \beta^{b}$ & $\begin{array}{c}\text { 6-a-acyl- } \beta \text {-glycoside } \\
(\%)^{b}\end{array}$ \\
\hline 1 & DMF & 0.386 & 30 & 1.0 & 54 & $48 / 51$ & - \\
\hline 2 & THF & 0.207 & 30 & 1.0 (grinded) & 11 & $2 / 98$ & 24 ( $\beta$ only) \\
\hline 3 & toluene & 0.099 & 30 & 1.0 (grinded) & - & - & 3 ( $\beta$ only) \\
\hline 4 & 1,4-dioxane & 0.164 & 10 & 1.0 (grinded) & 51 & $1 / 99$ & 7 ( $\beta$ only) \\
\hline 5 & 1,4-dioxane & 0.164 & 10 & 3.0 (grinded) & $66^{c}$ & $2 / 98$ & - \\
\hline
\end{tabular}

${ }^{a}$ normalized polatiry parameter. ${ }^{b}$ NMR yield using 1,3-dinitrobenzene as an internal standard. ${ }^{c}$ ssolated yield 


\section{Mechanistic insights using epimerized D-glucose (Scheme S2)}

A solution of $\alpha$-D-glucose in $\mathrm{MeOH}$ (with catalytic amount of TFA) was stirred at $\mathrm{rt}$ for 20-24 $\mathrm{h}$ and concentrated in vacuo to give a white powder. The powder was dried well to give a partially epimerized D-glucose $\left(\alpha / \beta=78 / 22,51 / 49\right.$; see below ${ }^{1} \mathrm{H}$ NMR spectra) as a white powder. Model reactions were examined using these epimerized D-glucose as a glycosyl donor. A decrease in the $\alpha$-anomer content in the substrate resulted in a decrease in the $\beta$-anomer content of the glycoside. These results indicated that the $\beta$-glycoside was generated selectively from $\alpha$-D-glucose.

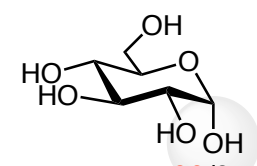

$\alpha / \beta=100 / 0$

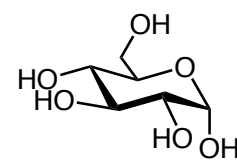

$\alpha / \beta=100 / 0$

$\mathrm{MeOH}(0.04 \mathrm{M})$

rt, $20 \mathrm{~h}$
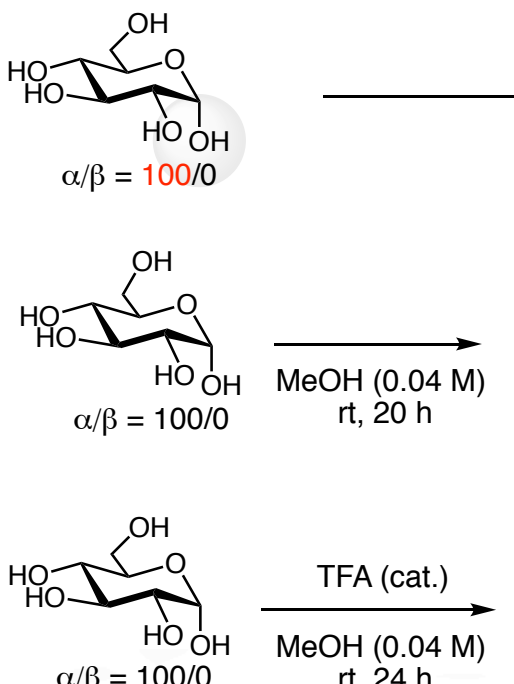

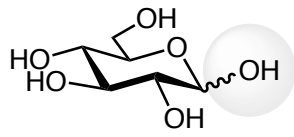

$\alpha / \beta=78 / 22$

3.0 equiv

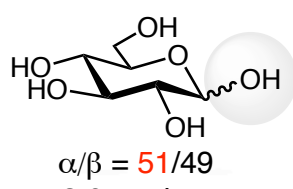

3.0 equiv

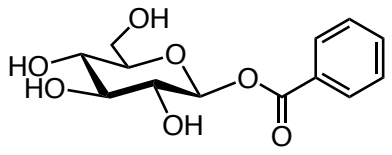

$66 \%, \alpha / \beta=2 / 98$
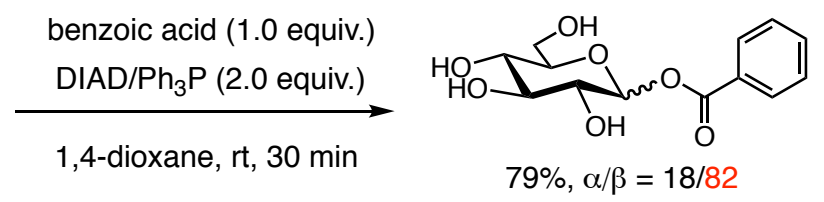

$79 \%, \alpha / \beta=18 / 82$

benzoic acid (1.0 equiv.) $\mathrm{DIAD} / \mathrm{Ph}_{3} \mathrm{P}$ (2.0 equiv.)

1,4-dioxane, rt, $30 \mathrm{~min}$

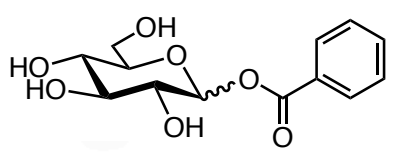

$76 \%, \alpha / \beta=38 / 62$

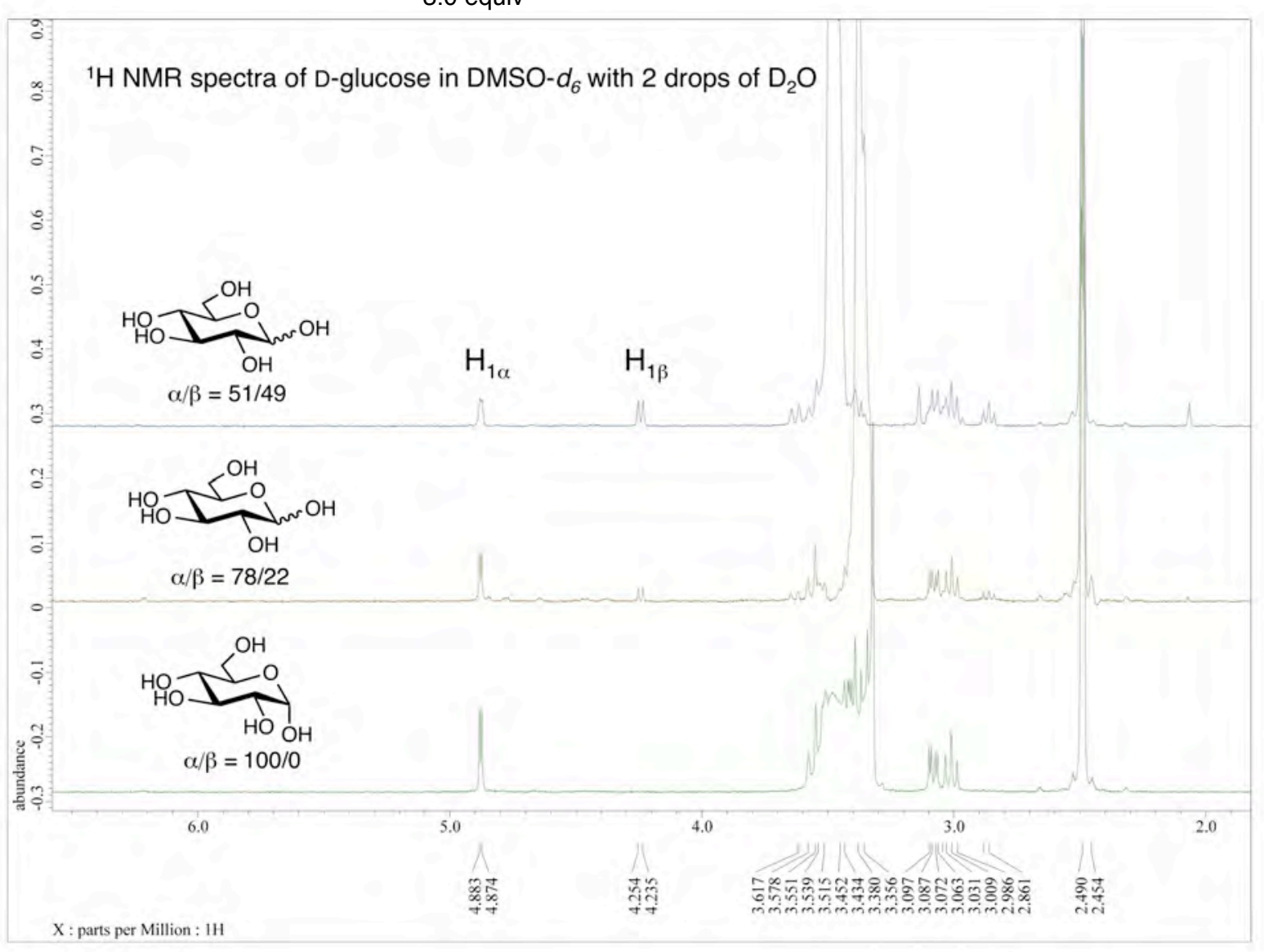




\section{Specific procedures and characterization data of the glycosides}

\section{1-O-Benzoyl- $\beta$-D-glucopyranoside. (representative example)}

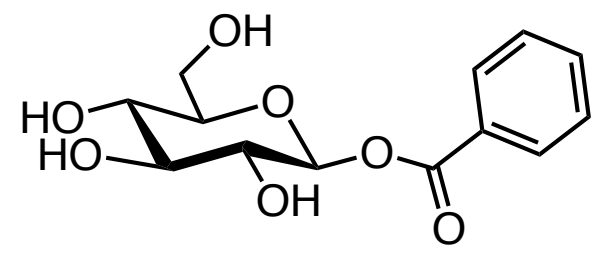

A round-bottom flask was charged with powdered $\alpha$-D-glucose $(2.00 \mathrm{~g}, 11 \mathrm{mmol}, 3.0$ equiv. $)$ and dehydrated 1,4-dioxane $(222 \mathrm{~mL})$ under an Ar atmosphere. After ultrasound irradiation of the resulting suspension for $15 \mathrm{~min}$, benzoic acid (452 mg, $3.7 \mathrm{mmol}, 1.0$ equiv.) and $\mathrm{Ph}_{3} \mathrm{P}$ (5.82 g, $22 \mathrm{mmol}, 6.0$ equiv.) were added. To the mixture was added DIAD $(4.40 \mathrm{~mL}, 22 \mathrm{mmol}, 6.0$ equiv.) dropwise by syringe and the resulting mixture was stirred vigorously at $\mathrm{rt}$ for $30 \mathrm{~min}$. The reaction mixture was quenched with $\mathrm{MeOH}$, stirred for $5 \mathrm{~min}$, and concentrated in vacuo at $40^{\circ} \mathrm{C}$ (water bath) to give a residue. The residue was directly purified by flash column chromatography $\left(\mathrm{SiO}_{2}, \mathrm{CHCl}_{3} / \mathrm{MeOH} 50: 1\right.$ to $\left.5: 1 \mathrm{v} / \mathrm{v}\right)$ to give the $\beta$-glycoside (694 $\mathrm{mg}, 66 \%, \alpha / \beta=2 / 98)$ as a white powder.

Analytical data: m.p. $188-191{ }^{\circ} \mathrm{C} ;[\boldsymbol{\alpha}]_{\mathbf{D}}{ }^{21}=-5.6\left(c\right.$ 0.71, acetone); $\mathbf{T L C}\left(\mathrm{CHCl}_{3} / \mathrm{MeOH} 5: 1 \mathrm{v} / \mathrm{v}\right): \mathrm{R}_{f}=0.17$; ${ }^{1}$ H NMR (400 MHz, acetone- $\left.d_{6}+\mathrm{D}_{2} \mathrm{O}\right) \delta: 8.10-8.07(\mathrm{~m}, 2 \mathrm{H}), 7.69-7.66(\mathrm{~m}, 1 \mathrm{H}), 7.56-7.52(\mathrm{~m}, 2 \mathrm{H}), 5.73(\mathrm{~d}$, $J=8.4 \mathrm{~Hz}, 1 \mathrm{H}), 3.82$ (dd, $J=11.6,1.6 \mathrm{~Hz}, 1 \mathrm{H}), 3.68$ (dd, $J=12.0,5.2 \mathrm{~Hz}, 1 \mathrm{H}), 3.55-3.45(\mathrm{~m}, 4 \mathrm{H}) ;{ }^{13} \mathrm{C}$ NMR $\left(100 \mathrm{MHz}, \mathrm{DMSO}-d_{6}\right) \delta: 164.7,133.9,129.6,129.2,128.8,95.0,78.0,76.4,72.5,69.5,60.6$; IR $(\mathrm{KBr}$, $\mathrm{cm}^{-1}$ ): 3550, 3307, 2925, 1714, 1594, 1451, 1395, 1316, 1281, 1077, 1021, 711; HRMS-ESI $(\mathrm{m} / \mathrm{z})$ : Calcd. for $\mathrm{C}_{13} \mathrm{H}_{16} \mathrm{O}_{7} \mathrm{Cl}[\mathrm{M}+\mathrm{Cl}]^{-}$319.0579; found, 319.0575 .

\section{1-O-(2,6-Dimethylbenzoyl)- $\beta$-D-glucopyranoside (2a).}<smiles>Cc1cccc(C)c1C(=O)OC1OC(CO)C(O)[C@H](O)[C@H]1O</smiles>

General procedure A for the synthesis of glycoside was followed, starting with $\alpha$-D-glucose (200 mg, 1.1 mmol, 3.0 equiv.), 2,6-dimethylbenzoic acid (55.6 mg, $0.37 \mathrm{mmol}, 1.0$ equiv.), DIAD (146 $\mu \mathrm{L}, 0.74 \mathrm{mmol}$, 2.0 equiv.), and $\mathrm{Ph}_{3} \mathrm{P}$ (194 mg, $0.74 \mathrm{mmol}, 2.0$ equiv.) in 1,4-dioxane (37 mL) for $30 \mathrm{~min}$, affording glucoside 2a (106 mg, 92\%, $\alpha / \beta=1 / 99)$ as a white amorphous powder after purification by flash chromatography, eluting with $\mathrm{CHCl}_{3} / \mathrm{MeOH}(50: 1$ to $5: 1 \mathrm{v} / \mathrm{v})$. Analytical data: $[\alpha]_{\mathbf{D}}{ }^{21}=+4.1$ (c 1.0, acetone); TLC $\left(\mathrm{CHCl}_{3} / \mathrm{MeOH} 5: 1 \mathrm{v} / \mathrm{v}\right): \mathrm{R}_{f}=0.30 ;{ }^{1} \mathbf{H}$ NMR $\left(400 \mathrm{MHz}\right.$, acetone- $\left.d_{6}+\mathrm{D}_{2} \mathrm{O}\right) \delta: 7.23(\mathrm{t}, J=8.0 \mathrm{~Hz}, 1 \mathrm{H})$, $7.08(\mathrm{~d}, J=7.6 \mathrm{~Hz}, 2 \mathrm{H}), 5.76(\mathrm{~d}, J=8.4 \mathrm{~Hz}, 1 \mathrm{H}), 3.85(\mathrm{dd}, J=12.0,2.8 \mathrm{~Hz}, 1 \mathrm{H}), 3.71(\mathrm{dd}, J=12.0,4.4 \mathrm{~Hz}$, $1 \mathrm{H}), 3.54(\mathrm{t}, J=8.4 \mathrm{~Hz} 1 \mathrm{H}), 3.52-3.48(\mathrm{~m}, 1 \mathrm{H}), 3.45-3.37(\mathrm{~m}, 2 \mathrm{H}), 2.32(\mathrm{~s}, 6 \mathrm{H}) ;{ }^{13} \mathbf{C}$ NMR $(150 \mathrm{MHz}$, acetone- $\left.d_{6}\right) \delta: 168.9,135.9,134.6,130.3,128.3,95.8,78.5,78.1,73.8,71.3,62.6,19.7 ; \mathbf{I R}\left(\mathrm{KBr}, \mathrm{cm}^{-1}\right): 3570$, 
3355, 2937, 2861, 1750, 1706, 1468, 1427, 1260, 1244, 1101, 1077, 1057, 1-34, 894, 782.; HRMS-ESI ${ }^{-}$ $(\mathrm{m} / \mathrm{z})$ : Calcd. for $\mathrm{C}_{15} \mathrm{H}_{20} \mathrm{O}_{7} \mathrm{Cl}[\mathrm{M}+\mathrm{Cl}]^{-}$347.0896; found, 347.0903.

\section{1-O-(2,6-Dimethylbenzoyl)- $\alpha$-D-glucopyranoside (2b).}<smiles>Cc1cccc(C)c1C(=O)OC(O)[C@H](O)[C@H](O)C(O)CO</smiles>

General procedure A for the synthesis of glycoside was followed, starting with $\beta$-D-glucose (purchased from TCI, $\alpha / \beta=4: 96,105 \mathrm{mg}, 0.58 \mathrm{mmol}, 3.0$ equiv.), 2,6-dimethylbenzoic acid (29.0 mg, $0.19 \mathrm{mmol}, 1.0$ equiv.), DIAD (70.0 $\mu \mathrm{L}, 0.39 \mathrm{mmol}, 2.0$ equiv.), and $\mathrm{Ph}_{3} \mathrm{P}$ (101 mg, $0.39 \mathrm{mmol}, 2.0$ equiv.) in 1,4-dioxane (19 $\left.\mathrm{mL}\right)$ for $30 \mathrm{~min}$, affording glycoside $\mathbf{2 b}(57.0 \mathrm{mg}, 94 \%, \alpha / \beta=89: 11)$ as a white amorphous powder after purification by flash chromatography, eluting with $\mathrm{CHCl}_{3} / \mathrm{MeOH}(10: 1$ to $5: 1 \mathrm{v} / \mathrm{v})$. Analytical data: $[\alpha]_{\mathbf{D}}{ }^{{ }^{20}}=$ +104 ( $c$ 0.5, acetone); TLC $\left(\mathrm{CHCl}_{3} / \mathrm{MeOH} 5 / 1 \mathrm{v} / \mathrm{v}\right): \mathrm{R}_{f}=0.22 ;{ }^{1} \mathbf{H}$ NMR (400 MHz, acetone- $\left.d_{\sigma}+\mathrm{D}_{2} \mathrm{O}\right) \delta$ : $7.21(\mathrm{t}, J=7.8 \mathrm{~Hz}, 1 \mathrm{H}), 7.05(\mathrm{~d}, J=7.8 \mathrm{~Hz}, 2 \mathrm{H}), 6.38(\mathrm{~d}, J=3.7 \mathrm{~Hz}, 1 \mathrm{H}), 3.80-3.60(\mathrm{~m}, 5 \mathrm{H}), 3.57-3.38(\mathrm{~m}$, $1 \mathrm{H}), 2.31(\mathrm{~s}, 6 \mathrm{H}) ;{ }^{13} \mathbf{C}$ NMR $\left(100 \mathrm{MHz}\right.$, acetone- $\left.d_{\sigma}+\mathrm{D}_{2} \mathrm{O}\right) \delta: 169.5,135.3,134.9,130.1,128.1,93.6,76.1$, 74.4, 71.6, 70.4, 61.9, 19.6; IR (KBr, cm $\left.{ }^{-1}\right): 3346,2911,2334,1744,1464,1265,1080,776$; HRMS-ESI ${ }^{+}$ $(\mathrm{m} / \mathrm{z})$ : Calcd. for $\mathrm{C}_{15} \mathrm{H}_{20} \mathrm{O}_{7} \mathrm{Na}[\mathrm{M}+\mathrm{Na}]^{+}$335.1101; found, 335.1115 .

\section{1-O-(2,6-Dimethylbenzoyl)- $\beta$-D-galactopyranoside. (2c).}

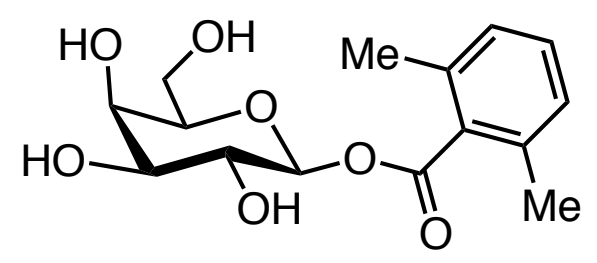

Modified general procedure A for the synthesis of glycoside was followed, starting with $\alpha$-D-galactose (purchased from TCI, $\alpha / \beta=96: 4,101 \mathrm{mg}, 0.56 \mathrm{mmol}, 3.0$ equiv.), 2,6-dimethylbenzoic acid (28.1 mg, 0.19 mmol, 1.0 equiv.), DIAD (70.0 $\mu \mathrm{L}, 0.37 \mathrm{mmol}, 2.0$ equiv.), and $\mathrm{Ph}_{3} \mathrm{P}$ (98.1 mg, $0.37 \mathrm{mmol}, 2.0$ equiv.) in 1,4-dioxane $(56 \mathrm{~mL})$ for $30 \mathrm{~min}$. The reaction mixture was quenched with $3 \mathrm{~mL}$ of $\mathrm{MeOH}$, stirred for $5 \mathrm{~min}$, and concentrated in vacuo at $40{ }^{\circ} \mathrm{C}$ (water bath) to give a residue. The residue was directly purified by flash column chromatography $\left(\mathrm{SiO}_{2}, \mathrm{CHCl}_{3} / \mathrm{MeOH} 10: 1\right.$ to $\left.5: 1 \mathrm{v} / \mathrm{v}\right)$ to give $2 \mathrm{c}(33.6 \mathrm{mg}, 58 \%, \alpha / \beta=2: 98)$ as a white amorphous powder. Analytical data: $[\alpha]_{\mathbf{D}}{ }^{19}=+17(c 0.5, \mathrm{MeOH}) ;$ TLC $\left(\mathrm{CHCl}_{3} / \mathrm{MeOH} 5 / 1 \mathrm{v} / \mathrm{v}\right)$ : $\mathrm{R}_{f}=0.17 ;{ }^{1} \mathbf{H}$ NMR (400 MHz, acetone- $\left.d_{6}+\mathrm{D}_{2} \mathrm{O}\right) \delta: 7.21(\mathrm{t}, J=7.8 \mathrm{~Hz}, 1 \mathrm{H}), 7.05(\mathrm{~d}, J=7.8 \mathrm{~Hz}, 2 \mathrm{H}), 5.69(\mathrm{~d}$, $J=7.8 \mathrm{~Hz}, 1 \mathrm{H}), 3.98(\mathrm{~d}, J=3.2 \mathrm{~Hz}, 1 \mathrm{H}), 3.78-3.64(\mathrm{~m}, 5 \mathrm{H}), 2.29(\mathrm{~s}, 6 \mathrm{H}) ;{ }^{13} \mathrm{C}$ NMR (100 MHz, acetone- $\left.d_{6}+\mathrm{D}_{2} \mathrm{O}\right) \delta$ : 169.4, 135.6, 134.3, 130.2, 128.1, 96.1, 76.9, 74.4, 70.6, 69.0, 61.3, 19.5; IR (KBr, $\left.\mathrm{cm}^{-1}\right): 3409,2947,1748,1260,1076,769$; HRMS-ESI $^{+}(\mathrm{m} / \mathrm{z})$ : Calcd. for $\mathrm{C}_{15} \mathrm{H}_{20} \mathrm{O}_{7} \mathrm{Na}[\mathrm{M}+\mathrm{Na}]^{+} 335.1101$; found, 335.1117. 
1-O-(2,6-Dimethylbenzoyl)- $\beta$-D-xylopyranoside. (2d).

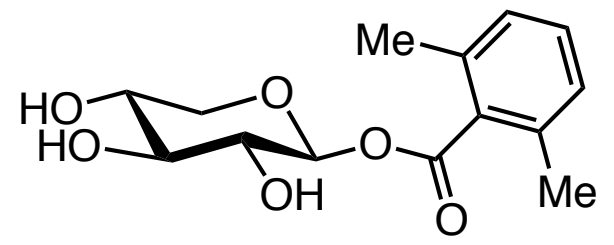

General procedure A for the synthesis of glycoside was followed, starting with $\alpha$-D-xylose (purchased from TCI, $\alpha / \beta=100 / 0,300 \mathrm{mg}, 2.0 \mathrm{mmol}, 3.0$ equiv.), 2,6-dimethylbenzoic acid (100 mg, $0.67 \mathrm{mmol}, 1.0$ equiv.), DIAD (262 $\mu \mathrm{L}, 1.3 \mathrm{mmol}, 2.0$ equiv.), $\mathrm{Ph}_{3} \mathrm{P}$ (349 mg, $1.3 \mathrm{mmol}, 2.0$ equiv.) in 1,4-dioxane (67 mL) for 30 min, affording $2 \mathbf{d}(99.6 \mathrm{mg}, 53 \%, \alpha / \beta=4 / 96)$ as a white amorphous powder after purification by flash chromatography, eluting with $\mathrm{CHCl}_{3} / \mathrm{MeOH}(20: 1$ to $10: 1 \mathrm{v} / \mathrm{v})$. Analytical data: $[\boldsymbol{\alpha}]_{\mathbf{D}}{ }^{21}=-5.9$ (c 0.62 , $\mathrm{MeOH}) ;$ TLC $\left(\mathrm{CHCl}_{3} / \mathrm{MeOH} 5: 1 \mathrm{v} / \mathrm{v}\right): \mathrm{R}_{f}=0.32 ;{ }^{1} \mathbf{H}$ NMR $\left(400 \mathrm{MHz}\right.$, acetone- $\left.d_{6}+\mathrm{D}_{2} \mathrm{O}\right) \delta: 7.23(\mathrm{t}, J=7.2$ $\mathrm{Hz}, 1 \mathrm{H}), 7.08$ (d, $J=7.6 \mathrm{~Hz}, 2 \mathrm{H}), 5.70(\mathrm{~d}, J=7.2 \mathrm{~Hz}, 1 \mathrm{H}), 3.94$ (dd, $J=11.6,4.8 \mathrm{~Hz}, 1 \mathrm{H}), 3.60-3.54$ (m, 1H), $3.51(\mathrm{t}, J=8.0 \mathrm{~Hz}, 1 \mathrm{H}), 3.43-3.38(\mathrm{~m}, 2 \mathrm{H}), 2.30$ (s, 6H); ${ }^{13} \mathbf{C}$ NMR (150 MHz, acetone- $\left.d_{6}\right) \delta: 168.9$, 135.7, 134.7, 130.3, 128.3, 96.5, 77.5, 73.3, 70.6, 67.3, 19.6; IR (KBr, cm $\left.{ }^{-1}\right): 3303,2921,2893,1746,1591$, 1471, 1427, 1363, 1264, 1240, 1089, 1049, 770; HRMS-FAB ${ }^{-}(m / z)$ : Calcd. for $\mathrm{C}_{14} \mathrm{H}_{17} \mathrm{O}_{6}[\mathrm{M}-\mathrm{H}]^{-}$ 281.1025; found, 281.1018 .

1-O-(2,6-Dimethylbenzoyl)- $\alpha$-D-arabinopyranoside (2e).<smiles>Cc1cccc(C)c1C(=O)OC1OCC(O)C[C@H]1O</smiles>

General procedure A for the synthesis of glycoside was followed, starting with $\alpha$-D-xylose (purchased from TCI, $\alpha / \beta=4 / 96,90 \mathrm{mg}, 0.60 \mathrm{mmol}, 3.0$ equiv.), 2,6-dimethylbenzoic acid (30 mg, $0.20 \mathrm{mmol}, 1.0$ equiv.), DIAD (79.0 $\mu \mathrm{L}, 0.40 \mathrm{mmol}, 2.0$ equiv.), $\mathrm{Ph}_{3} \mathrm{P}$ (105 mg, $0.40 \mathrm{mmol}, 2.0$ equiv.) in 1,4-dioxane (20 mL) for $30 \mathrm{~min}$, affording $2 \mathrm{e}(49.1 \mathrm{mg}, 87 \%, \alpha / \beta=95 / 5)$ as a white amorphous powder after purification by flash chromatography, eluting with $\mathrm{CHCl}_{3} / \mathrm{MeOH}(20: 1$ to $10: 1 \mathrm{v} / \mathrm{v})$. Analytical data: $[\boldsymbol{\alpha}]_{\mathbf{D}}{ }^{\mathbf{1 9}}=-20($ c $1.0, \mathrm{MeOH})$; TLC $\left(\mathrm{CHCl}_{3} / \mathrm{MeOH} 5: 1 \mathrm{v} / \mathrm{v}\right): \mathrm{R}_{f}=0.35 ;{ }^{1} \mathbf{H}$ NMR $\left(400 \mathrm{MHz}\right.$, acetone- $\left.d_{6}+\mathrm{D}_{2} \mathrm{O}\right) \delta: 7.21(\mathrm{t}, J=7.6 \mathrm{~Hz}, 1 \mathrm{H})$, $7.06(\mathrm{~d}, J=7.6 \mathrm{~Hz}, 2 \mathrm{H}), 5.68(\mathrm{~d}, J=6.8 \mathrm{~Hz}, 1 \mathrm{H}), 3.96-3.88(\mathrm{~m}, 2 \mathrm{H}), 3.79-3.67(\mathrm{~m}, 3 \mathrm{H}), 2.30(\mathrm{~s}, 6 \mathrm{H}) ;{ }^{13} \mathbf{C}$ NMR (100 MHz, acetone- $d_{6}$ ) $\delta: 168.7,135.1,134.3,129.8,127.8,95.8,73.1,70.4,67.9,66.5,19.2$; IR (neat, $\left.\mathrm{cm}^{-1}\right)$ : 3389, 1744, 1260, 1243, 1055; HRMS-ESI ${ }^{+}(\mathrm{m} / \mathrm{z})$ : Calcd. for $\mathrm{C}_{14} \mathrm{H}_{18} \mathrm{O}_{6} \mathrm{Na}[\mathrm{M}+\mathrm{Na}]^{+}$305.0996; found, 305.0985 . 
1-O-(2,6-Dimethylbenzoyl)- $\beta$-D-mannopyranoside (2f).

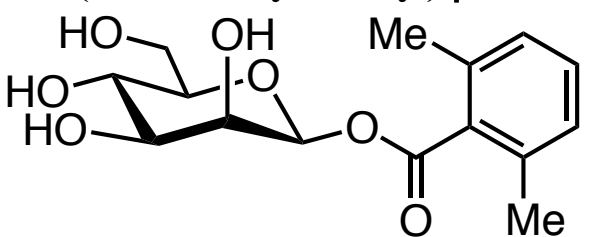

Modified general procedure A for the synthesis of glycoside was followed, starting with $\alpha$-D-mannose (purchased from TCI, $\alpha / \beta=100 / 0,100 \mathrm{mg}, 0.56 \mathrm{mmol}, 3.0$ equiv.), 2,6-dimethylbenzoic acid (27.8 $\mathrm{mg}, 0.19$ mmol, 1.0 equiv.), DIAD (109 $\mu \mathrm{L}, 0.56 \mathrm{mmol}, 3.0$ equiv.), $\mathrm{Ph}_{3} \mathrm{P}$ (146 mg, $0.56 \mathrm{mmol}, 3.0$ equiv.) in 1,4-dioxane $(10 \mathrm{~mL})$ for $30 \mathrm{~min}$. The reaction mixture was quenched with $2 \mathrm{~mL}$ of $\mathrm{MeOH}$, stirred for 10 min, and concentrated in vacuo at $40{ }^{\circ} \mathrm{C}$ (water bath) to give a residue. The residue was directly purified by flash column chromatography $\left(\mathrm{SiO}_{2}, \mathrm{CHCl}_{3} / \mathrm{MeOH} 50: 1\right.$ to $5: 1 \mathrm{v} / \mathrm{v}$ ) to give the mannopyranoside (38.2 $\mathrm{mg}$, $66 \%, \alpha / \beta=13 / 87$ ) as a pale yellow amorphous powder. Pure $2 \mathrm{f}$ was isolated by recrystallization from $\mathrm{CH}_{3} \mathrm{CN}$ as a colorless prism crystal $(\alpha / \beta=0 / 100)$. Analytical data: m.p. $183-185{ }^{\circ} \mathrm{C} ;[\boldsymbol{\alpha}]_{\mathbf{D}}{ }^{21}=+12(c 0.25$, $\mathrm{MeOH}) ;$ TLC $\left(\mathrm{CHCl}_{3} / \mathrm{MeOH} 5: 1 \mathrm{v} / \mathrm{v}\right): \mathrm{R}_{f}=0.39 ;{ }^{1} \mathbf{H}$ NMR $\left(400 \mathrm{MHz}\right.$, methanol- $\left.d_{4}\right) \delta: 7.22(\mathrm{t}, J=7.6 \mathrm{~Hz}$, 1H), 7.06 (d, $J=7.6 \mathrm{~Hz}, 2 \mathrm{H}), 5.96$ (br s, $1 \mathrm{H}), 4.02$ (d, $J=2.4 \mathrm{~Hz}, 1 \mathrm{H}), 3.90$ (dd, $J=12.4,2.4 \mathrm{~Hz}, 1 \mathrm{H}), 3.78$ (dd, $J=12.0,5.2 \mathrm{~Hz}, 1 \mathrm{H}), 3.70-3.60(\mathrm{~m}, 2 \mathrm{H}), 3.44-3.40(\mathrm{~m}, 1 \mathrm{H}), 2.35(\mathrm{~s}, 6 \mathrm{H}) ;{ }^{13} \mathrm{C}$ NMR (100 MHz, methanol- $\left.d_{4}\right) \delta$ : 169.6, 136.5, 134.3, 130.8, 128.6, $94.9\left(J_{\mathrm{C}-\mathrm{H}}=162 \mathrm{~Hz}\right), 79.4,75.0,71.7,67.9,62.6,19.9$; IR $\left(\mathrm{KBr}, \mathrm{cm}^{-1}\right): 3463,3244,2885,1746,1591,1427,1268,1065,1010,890,782$; HRMS-ESI $^{+}(\mathrm{m} / \mathrm{z})$ : Calcd. for $\mathrm{C}_{15} \mathrm{H}_{20} \mathrm{O}_{7} \mathrm{Na}[\mathrm{M}+\mathrm{Na}]^{+}$335.1101; found, 335.1114 .

\section{1-O-Octanoyl- $\beta$-D-glucopyranoside (4a).}<smiles>CCCCCCC(=O)OC(O)[C@H](O)C(CO)CO</smiles>

General procedure A for the synthesis of glycoside was followed, starting with $\alpha$-D-glucose (270 mg, 1.5 mmol, 3.0 equiv.), octanoic acid (79.2 $\mu \mathrm{L}, 0.50 \mathrm{mmol}, 1.0$ equiv.), DIAD (197 $\mu \mathrm{L}, 1.0 \mathrm{mmol}, 2.0$ equiv.), and $\mathrm{Ph}_{3} \mathrm{P}$ (262 mg, $1.0 \mathrm{mmol}, 2.0$ equiv.) in 1,4-dioxane $(50 \mathrm{~mL}$ ) for $30 \mathrm{~min}$, affording the glucoside (101 $\mathrm{mg}, 66 \%, \alpha / \beta=2 / 98)$ as a white amorphous powder after purification by flash chromatography, eluting with $\mathrm{CHCl}_{3} / \mathrm{MeOH}\left(50: 1\right.$ to 5:1 v/v). Analytical data: $[\alpha]_{\mathbf{D}}{ }^{21}=-0.7$ (c 1.0, $\left.\mathrm{MeOH}\right) ; \mathbf{T L C}\left(\mathrm{CHCl}_{3} / \mathrm{MeOH} 5: 1 \mathrm{v} / \mathrm{v}\right)$ : $\mathrm{R}_{f}=0.36 ;{ }^{1} \mathbf{H}$ NMR (400 MHz, acetone- $\left.d_{6}\right) \delta: 5.50(\mathrm{~d}, J=8.2 \mathrm{~Hz}, 1 \mathrm{H}), 4.48(\mathrm{~d}, J=4.6 \mathrm{~Hz}, 1 \mathrm{H}), 4.37(\mathrm{~d}, J$ $=4.1 \mathrm{~Hz}, 1 \mathrm{H}), 4.26(\mathrm{~d}, J=4.1 \mathrm{~Hz}, 1 \mathrm{H}), 3.83-3.78(\mathrm{~m}, 1 \mathrm{H}), 3.70-3.64(\mathrm{~m}, 1 \mathrm{H}), 3.59(\mathrm{t}, J=6.2 \mathrm{~Hz}, 1 \mathrm{H}) 3.50-$ $3.29(\mathrm{~m}, 4 \mathrm{H}), 2.35$ (dt, $J=7.4,2.3 \mathrm{~Hz}, 2 \mathrm{H}), 1.61$ (quint, $J=7.3 \mathrm{~Hz}, 2 \mathrm{H}), 1.38-1.26(\mathrm{~m}, 8 \mathrm{H}), 0.88(\mathrm{t}, J=6.9$ $\mathrm{Hz}, 3 \mathrm{H}) ;{ }^{13} \mathbf{C}$ NMR (100 MHz, methanol- $\left.d_{4}\right) \delta: 174.1,95.5,78.7,77.9,73.9,71.0,62.2,34.8,32.8,30.07$, 30.05, 25.6, 23.6, 14.4; IR (KBr, cm $\left.{ }^{-1}\right): 3359,2932,2857,1734,1464,1359,1179$, 885, 495; HRMS-ESI ${ }^{+}$ $(\mathrm{m} / \mathrm{z})$ : Calcd. for $\mathrm{C}_{14} \mathrm{H}_{2} \mathrm{O}_{7} \mathrm{Na}[\mathrm{M}+\mathrm{Na}]^{+} 329.1571$; found, 329.1576 . 
1-O-Leulinoyl- $\beta$-D-glucopyranoside (4b).

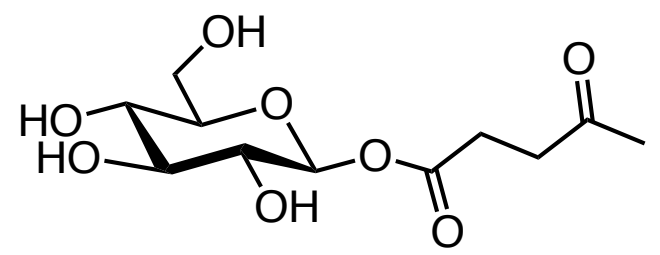

General procedure A for the synthesis of glycoside was followed, starting with $\alpha$-D-glucose (650 mg, 3.6 mmol, 3.0 equiv.), 4-oxopentanoic acid (140 mg, 1.2 mmol, 1.0 equiv.), DIAD (474 $\mu \mathrm{L}, 2.4$ mmol, 2.0 equiv.), and $\mathrm{Ph}_{3} \mathrm{P}$ (630 mg, $2.4 \mathrm{mmol}, 2.0$ equiv.) in 1,4-dioxane (120 mL) for $30 \mathrm{~min}$, affording the glucoside (248 $\mathrm{mg}, 74 \%, \alpha / \beta=2 / 98)$ as a white amorphous powder after purification by flash chromatography, eluting with $\mathrm{CHCl}_{3} / \mathrm{MeOH}(50: 1$ to $5: 1 \mathrm{v} / \mathrm{v})$. Analytical data: $[\alpha]_{\mathbf{D}}{ }^{21}=-2.1($ c $1.0, \mathrm{MeOH})$; TLC $\left(\mathrm{CHCl}_{3} / \mathrm{MeOH} 5: 1 \mathrm{v} / \mathrm{v}\right): \mathrm{R}_{f}=0.27 ;{ }^{1} \mathbf{H}$ NMR $\left(400 \mathrm{MHz}\right.$, acetone- $\left.d_{6}+\mathrm{D}_{2} \mathrm{O}\right) \delta: 5.45(\mathrm{~d}, J=8.3 \mathrm{~Hz}, 1 \mathrm{H})$, 3.81-3.76 (m, 1H), 3.67-3.62 (m, 1H), 3.48-3.29 (m, 4H), $2.78(\mathrm{t}, J=6.4 \mathrm{~Hz}, 2 \mathrm{H}), 2.66-2.52(\mathrm{~m}, 2 \mathrm{H}), 2.14$ (s, 3H); ${ }^{13}$ C NMR (100 MHz, methanol- $\left.d_{4}\right) \delta: 209.5,173.3,95.7,78.7,77.8,73.8,70.9,62.3,38.3,29.7$, 28.8; IR (KBr, cm $\left.{ }^{-1}\right)$ : 3358, 2932, 1750, 1710, 1513, 1421, 1367, 1171, 1065, 889; HRMS-ESI ${ }^{+}(\mathrm{m} / z)$ : Calcd. for $\mathrm{C}_{11} \mathrm{H}_{18} \mathrm{O}_{8} \mathrm{Na}[\mathrm{M}+\mathrm{Na}]^{+}$301.0894; found, 301.0900 .

1-O-(3-Phenylpropanoyl)- $\beta$-D-glucopyranoside (4c).<smiles>O=C(CCc1ccccc1)O[C@H](O)[C@H](O)[C@H](O)CO</smiles>

General procedure A for the synthesis of glycoside was followed, starting with $\alpha$-D-glucose $(270 \mathrm{mg}, 1.5$ mmol, 3.0 equiv.), 3-phenylpropanoic acid (75.1 mg, 0.50 mmol, 1.0 equiv.), DIAD (197 $\mu \mathrm{L}, 1.0 \mathrm{mmol}, 2.0$ equiv.), and $\mathrm{Ph}_{3} \mathrm{P}$ (262 mg, $1.0 \mathrm{mmol}, 2.0$ equiv.) in 1,4-dioxane (50 mL) for $30 \mathrm{~min}$, affording the glucoside (133 $\mathrm{mg}, 82 \%, \alpha / \beta=3 / 97$ ) as a white amorphous powder after purification by flash chromatography, eluting with $\mathrm{CHCl}_{3} / \mathrm{MeOH}\left(50: 1\right.$ to 5:1 v/v). Analytical data: $[\alpha]_{\mathbf{D}}{ }^{21}=+4.1(c 1.0, \mathrm{MeOH}) ; \mathbf{T L C}\left(\mathrm{CHCl}_{3} / \mathrm{MeOH} 5: 1\right.$ $\mathrm{v} / \mathrm{v}): \mathrm{R}_{f}=0.38 ;{ }^{1} \mathbf{H}$ NMR (400 MHz, acetone- $\left.d_{6}\right) \delta: 7.29-7.19(\mathrm{~m}, 5 \mathrm{H}), 5.52(\mathrm{~d}, J=8.2 \mathrm{~Hz}, 1 \mathrm{H}), 4.48(\mathrm{~d}, J=$ $4.6 \mathrm{~Hz}, 1 \mathrm{H}), 4.38$ (d, $J=4.2 \mathrm{~Hz}, 1 \mathrm{H}), 4.27(\mathrm{~d}, J=3.7 \mathrm{~Hz}, 1 \mathrm{H}), 3.84-3.79(\mathrm{~m}, 1 \mathrm{H}), 3.71-3.64(\mathrm{~m}, 1 \mathrm{H}), 3.58(\mathrm{t}$, $J=6.0 \mathrm{~Hz}, 1 \mathrm{H}), 3.51-3.31(\mathrm{~m}, 4 \mathrm{H}), 2.93(\mathrm{t}, J=7.8 \mathrm{~Hz}, 2 \mathrm{H}), 2.68(\mathrm{dt}, J=7.6,3.2 \mathrm{~Hz}, 2 \mathrm{H}) ;{ }^{13} \mathbf{C} \mathbf{~ N M R}(100$ MHz, acetone- $d_{6}$ ) $\delta: 171.9,141.6,129.2,127.0,95.3,78.3,77.8,73.8,71.0,62.4,36.3,31.1$ (One sp ${ }^{2}$ carbon signal missing, possibly due to signal overlap.); IR (KBr, cm $\left.{ }^{-1}\right): 3347,2931,1754,1455,1373,1203,1076$, 896, 752, 699; HRMS-ESI ${ }^{+}$(m/z): Calcd. for $\mathrm{C}_{15} \mathrm{H}_{20} \mathrm{O}_{7} \mathrm{Na}$ [M+Na ${ }^{+}$335.1101; found, 335.1105 . 
1-O-\{(2S)-2-(9H-Fluoren-9-ylmethoxycarbonylamino)-3-methylbutanoyl\}- $\beta$-D-glucopyranoside (4d).<smiles>CC(C)[C@H](N)C(=O)OC1OC(CO)[C@@H](O)C(O)[C@H]1O</smiles>

General procedure A for the synthesis of glycoside was followed, starting with $\alpha$-D-glucose (108 mg, 0.60 mmol, 3.0 equiv.), Fmoc-Val-OH (67.9 mg, 0.20 mmol, 1.0 equiv.), DIAD (78.8 $\mu \mathrm{L}, 0.40$ mmol, 2.0 equiv.), and $\mathrm{Ph}_{3} \mathrm{P}$ (105 mg, $0.40 \mathrm{mmol}, 2.0$ equiv.) in 1,4-dioxane $(20 \mathrm{~mL})$ for $30 \mathrm{~min}$, affording the glucoside (88.5 $\mathrm{mg}, 88 \%, \alpha / \beta=2 / 98)$ as a white amorphous powder after purification by flash chromatography, eluting with $\mathrm{CHCl}_{3} / \mathrm{MeOH}(50: 1$ to $5: 1 \mathrm{v} / \mathrm{v})$. Analytical data: $[\alpha]_{\mathbf{D}}{ }^{21}=-44(c 0.5, \mathrm{MeOH}) ; \mathbf{T L C}\left(\mathrm{CHCl}_{3} / \mathrm{MeOH} 5: 1 \mathrm{v} / \mathrm{v}\right)$ : $\mathrm{R}_{f}=0.41 ;{ }^{1} \mathbf{H}$ NMR $\left(400 \mathrm{MHz}\right.$, methanol- $\left.d_{4}\right) \delta: 7.80(\mathrm{~d}, J=7.8 \mathrm{~Hz}, 2 \mathrm{H}), 7.68(\mathrm{t}, J=7.8 \mathrm{~Hz}, 2 \mathrm{H}), 7.39(\mathrm{t}, J=$ $7.8 \mathrm{~Hz}, 2 \mathrm{H}), 7.32$ (t, $J=7.8 \mathrm{~Hz}, 2 \mathrm{H}), 5.50$ (d, $J=7.8 \mathrm{~Hz}, 1 \mathrm{H}), 4.45-4.33(\mathrm{~m}, 2 \mathrm{H}), 4.28-4.17$ (m, 2H), 3.81 (dd, $J=11.9,1.8 \mathrm{~Hz}, 1 \mathrm{H}), 3.65(\mathrm{dd}, J=11.9,4.6 \mathrm{~Hz}, 1 \mathrm{H}), 3.45-3.33(\mathrm{~m}, 4 \mathrm{H}), 2.23$ (sext, $J=5.5 \mathrm{~Hz}, 1 \mathrm{H})$, $0.99(\mathrm{~d}, J=6.9 \mathrm{~Hz}, 3 \mathrm{H}), 0.95(\mathrm{~d}, J=6.9 \mathrm{~Hz}, 3 \mathrm{H}) ;{ }^{13} \mathbf{C}$ NMR (100 MHz, acetone- $\left.d_{6}\right) \delta: 171.8,157.6,144.9$, 144.7, 141.9, 128.5, 128.0, 127.9, 126.15, 126.08, 120.7, 95.9, 78.4, 77.2, 73.3, 70.7, 67.4, 62.1, 60.1, 47.8, 31.2, 19.4, 17.9; IR (KBr, cm $\left.{ }^{-1}\right): 3358,2965,1750,1702,1538,1513,1453,1250,1076,1027,738$; HRMS-ESI ${ }^{+}(\mathrm{m} / z)$ : Calcd. for $\mathrm{C}_{26} \mathrm{H}_{31} \mathrm{NO}_{9} \mathrm{Na}[\mathrm{M}+\mathrm{Na}]^{+}$524.1891; found, 524.1883.

\section{1-O-\{(3-Fluorophenyl)acetyl\}- $\beta$-D-glucopyranoside (4e).}

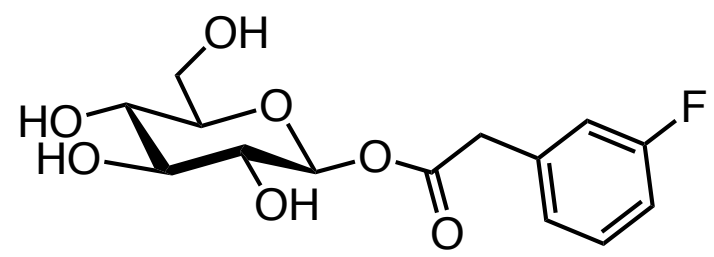

General procedure A for the synthesis of glycoside was followed, starting with $\alpha$-D-glucose (300 mg, 1.7 mmol, 3.0 equiv.), 3-fluorophenyl acetic acid ( $85.9 \mathrm{mg}, 0.58 \mathrm{mmol}, 1.0$ equiv.), DIAD (164 $\mu \mathrm{L}, 0.84 \mathrm{mmol}$, 2.0 equiv.), and $\mathrm{Ph}_{3} \mathrm{P}$ (219 mg, $0.84 \mathrm{mmol}, 2.0$ equiv.) in 1,4-dioxane (56 mL) for $30 \mathrm{~min}$, affording the glucoside (99.0 $\mathrm{mg}, 56 \%, \alpha / \beta=1 / 99)$ as a white amorphous powder after purification by flash chromatography, eluting with $\mathrm{CHCl}_{3} / \mathrm{MeOH}(50: 1$ to $5: 1 \mathrm{v} / \mathrm{v})$. Analytical data: $[\boldsymbol{\alpha}]_{\mathbf{D}}{ }^{21}=+8.3$ (c 0.81 , acetone); TLC $\left(\mathrm{CHCl}_{3} / \mathrm{MeOH} 5: 1 \mathrm{v} / \mathrm{v}\right): \mathrm{R}_{f}=0.24{ }^{1} \mathbf{H}$ NMR $\left(400 \mathrm{MHz}\right.$, acetone- $\left.d_{6}+\mathrm{D}_{2} \mathrm{O}\right) \delta: 7.39-7.34(\mathrm{~m}$, 1H), 7.17-7.13 (m, 2H), 7.07-7.02 (m, 1H), $5.53(\mathrm{~d}, J=8.0 \mathrm{~Hz}, 1 \mathrm{H}), 3.81-3.78(\mathrm{~m}, 3 \mathrm{H}), 3.66$ (dd, $J=12.8$, $4.8 \mathrm{~Hz}, 1 \mathrm{H}), 3.48-3.39(\mathrm{~m}, 3 \mathrm{H}), 3.35(\mathrm{t}, J=8.8 \mathrm{~Hz}, 1 \mathrm{H}){ }^{\mathbf{1 3}} \mathbf{C}$ NMR (150 MHz, acetone- $\left.d_{6}\right) \delta: 170.4,163.6$ $\left(\mathrm{d}, J_{\mathrm{CF}}=241.4 \mathrm{~Hz}\right), 137.7\left(\mathrm{~d}, J_{\mathrm{CF}}=8.7 \mathrm{~Hz}\right), 130.9\left(\mathrm{~d}, J_{\mathrm{CF}}=8.7 \mathrm{~Hz}\right), 126.4\left(\mathrm{~d}, J_{\mathrm{CF}}=2.9 \mathrm{~Hz}\right), 117.2\left(\mathrm{~d}, J_{\mathrm{CF}}=\right.$ $21.5 \mathrm{~Hz}), 114.5\left(\mathrm{~d}, J_{\mathrm{CF}}=21.6 \mathrm{~Hz}\right), 95.8,78.5,77.9,73.8,71.1,62.5,40.7$; IR $\left(\mathrm{KBr}, \mathrm{cm}^{-1}\right): 3606,3542,2937$, 1742, 1619, 1587, 1491, 1447, 1344, 1252, 1141, 1073, 1049, 1010, 894, 791, 679; HRMS-ESI ${ }^{-}(\mathrm{m} / \mathrm{z})$ : Calcd. for $\mathrm{C}_{14} \mathrm{H}_{17} \mathrm{O}_{7} \mathrm{FCl}[\mathrm{M}+\mathrm{Cl}]^{-}$351.0624; found, 351.0641 . 
1-O-(3-Bromobenzoyl)- $\beta$-D-glucopyranoside (4f).<smiles>O=C(OC1OC(CO)C(O)[C@H](O)[C@H]1O)c1cccc(Br)c1</smiles>

General procedure A for the synthesis of glycoside was followed, starting with $\alpha$-D-glucose $(270 \mathrm{mg}, 1.5$ mmol, 3.0 equiv.), 3-bromobenzoic acid (101 mg, 0.50 mmol, 1.0 equiv.), DIAD (197 $\mu \mathrm{L}, 1.0 \mathrm{mmol}, 2.0$ equiv.), and $\mathrm{Ph}_{3} \mathrm{P}$ (262 mg, $1.0 \mathrm{mmol}, 2.0$ equiv.) in 1,4-dioxane (50 mL) for $30 \mathrm{~min}$, affording the glucoside (128 mg, 70\%, $\alpha / \beta=1 / 99$ ) as a white amorphous powder after purification by flash chromatography, eluting with $\mathrm{CHCl}_{3} / \mathrm{MeOH}\left(50: 1\right.$ to 5:1 v/v). Analytical data: $[\alpha]_{\mathbf{D}}{ }^{20}=-19(c 1.0, \mathrm{MeOH}) ; \mathbf{T L C}\left(\mathrm{CHCl}_{3} / \mathrm{MeOH} 5: 1\right.$ $\mathrm{v} / \mathrm{v}): \mathrm{R}_{f}=0.42 ;{ }^{1} \mathbf{H}$ NMR (400 MHz, acetone- $\left.d_{6}+\mathrm{D}_{2} \mathrm{O}\right) \delta: 8.20(\mathrm{t}, J=1.8 \mathrm{~Hz}, 1 \mathrm{H}), 8.10-8.06(\mathrm{~m}, 1 \mathrm{H}), 7.88-$ $7.84(\mathrm{~m}, 1 \mathrm{H}), 7.52(\mathrm{t}, J=8.2 \mathrm{~Hz}, 1 \mathrm{H}), 5.72(\mathrm{~d}, J=8.2 \mathrm{~Hz}, 1 \mathrm{H}), 3.82(\mathrm{dd}, J=12.4,1.8 \mathrm{~Hz}, 1 \mathrm{H}), 3.68(\mathrm{dd}, J=$ 11.9, $5.0 \mathrm{~Hz}, 1 \mathrm{H}), 3.56-3.42(\mathrm{~m}, 4 \mathrm{H}),{ }^{13} \mathbf{C}$ NMR (100 MHz, acetone- $\left.d_{6}+\mathrm{D}_{2} \mathrm{O}\right) \delta: 164.3,137.3,133.2,132.7$, 131.6, 129.5, 122.9, 96.3, 78.6, 77.7, 73.8, 71.0, 62.4; IR (KBr, cm $\left.{ }^{-1}\right): 3343,1717,1429,1391,1288,1254$, 1079, 746, 646; HRMS-ESI ${ }^{-}(\mathrm{m} / \mathrm{z})$ : Calcd. for $\mathrm{C}_{13} \mathrm{H}_{15} \mathrm{BrO}_{7} \mathrm{Cl}[\mathrm{M}+\mathrm{Cl}]^{-}$396.9695; found, 396.9675.

1-O-(4-Fluorobenzoyl)- $\beta$-D-glucopyranoside (4g).<smiles>O=C(O[C@@H]1O[C@H](CO)[C@H](O)[C@H](O)[C@H]1O)c1ccc(F)cc1</smiles>

General procedure A for the synthesis of glycoside was followed, starting with $\alpha$-D-glucose (300 mg, 1.7 mmol, 3.0 equiv.), 4-fluorobenzoic acid (78.0 mg, 0.58 mmol, 1.0 equiv.), DIAD (164 $\mu \mathrm{L}, 0.84$ mmol, 2.0 equiv.), and $\mathrm{Ph}_{3} \mathrm{P}$ (219 mg, $0.84 \mathrm{mmol}, 2.0$ equiv.) in 1,4-dioxane (56 mL) for $30 \mathrm{~min}$, affording the glucoside $(89.3 \mathrm{mg}, 53 \%, \alpha / \beta=1 / 99)$ as a white amorphous powder after purification by flash chromatography, eluting with $\mathrm{CHCl}_{3} / \mathrm{MeOH}(50: 1$ to $5: 1 \mathrm{v} / \mathrm{v})$. Analytical data: $[\alpha]_{\mathbf{D}}{ }^{21}=-5.5$ (c 1.0, acetone); TLC $\left(\mathrm{CHCl}_{3} / \mathrm{MeOH} 5: 1 \mathrm{v} / \mathrm{v}\right): \mathrm{R}_{f}=0.22 ;{ }^{1} \mathbf{H}$ NMR $\left(400 \mathrm{MHz}\right.$, acetone- $\left.d_{6}+\mathrm{D}_{2} \mathrm{O}\right) \delta: 8.18-8.14(\mathrm{~m}, 2 \mathrm{H})$, $7.33-7.29(\mathrm{~m}, 2 \mathrm{H}), 5.72(\mathrm{~d}, J=8.0 \mathrm{~Hz}, 1 \mathrm{H}), 3.82(\mathrm{dd}, J=12.0,2.0 \mathrm{~Hz}, 1 \mathrm{H}), 3.68(\mathrm{dd}, J=12.0,4.4 \mathrm{~Hz}, 1 \mathrm{H})$, 3.58-3.45 (m, 4H); ${ }^{13} \mathbf{C}$ NMR $\left(150 \mathrm{MHz}\right.$, acetone- $\left.d_{6}\right) \delta: 166.9\left(\mathrm{~d}, J_{\mathrm{CF}}=249.9 \mathrm{~Hz}\right), 164.7,133.5\left(\mathrm{~d}, J_{\mathrm{CF}}=\right.$ $10.1 \mathrm{~Hz}), 127.2,116.5\left(\mathrm{~d}, J_{\mathrm{CF}}=23.0 \mathrm{~Hz}\right), 96.1,78.6,77.9,73.9,71.2,62.5 ; \mathbf{I R}\left(\mathrm{KBr}, \mathrm{cm}^{-1}\right): 3463,3331$, 2925, 1718, 1603, 1507, 1412, 1380, 1284, 1236, 1101, 1077, 858, 782; HRMS-ESI ${ }^{-}(\mathrm{m} / \mathrm{z})$ : Calcd. for $\mathrm{C}_{13} \mathrm{H}_{15} \mathrm{O}_{7} \mathrm{FCl}[\mathrm{M}+\mathrm{Cl}]^{-}$337.0469; found, 337.0485 . 
1-O-(2-Chloro-5-methylbenzoyl)- $\beta$-D-glucopyranoside (4h).<smiles>Cc1ccc(Cl)c(C(=O)OC(O)[C@H](O)[C@H](O)CCO)c1</smiles>

General procedure A for the synthesis of glycoside was followed, starting with $\alpha$-D-glucose (200 $\mathrm{mg}, 1.1$ mmol, 3.0 equiv.), 2-chloro-5-methylbenzoic acid (63.1 mg, $0.37 \mathrm{mmol}, 1.0$ equiv.), DIAD (146 $\mu \mathrm{L}, 0.74$ mmol, 2.0 equiv.), and $\mathrm{Ph}_{3} \mathrm{P}$ (194 mg, $0.74 \mathrm{mmol}, 2.0$ equiv.) in 1,4-dioxane (37 mL) for $30 \mathrm{~min}$, affording the glucoside $(73.0 \mathrm{mg}, 59 \%, \alpha / \beta=1 / 99)$ as a white amorphous powder after purification by flash chromatography, eluting with $\mathrm{CHCl}_{3} / \mathrm{MeOH}(50: 1$ to $5: 1 \mathrm{v} / \mathrm{v})$. Analytical data: $[\alpha]_{\mathbf{D}}{ }^{21}=-4.5$ (c 1.1, acetone); TLC $\left(\mathrm{CHCl}_{3} / \mathrm{MeOH} 5: 1 \mathrm{v} / \mathrm{v}\right): \mathrm{R}_{f}=0.26 ;{ }^{1} \mathbf{H}$ NMR $\left(400 \mathrm{MHz}\right.$, acetone- $\left.d_{6}+\mathrm{D}_{2} \mathrm{O}\right) \delta: 7.82(\mathrm{br} \mathrm{s}, 1 \mathrm{H}), 7.44$ $7.38(\mathrm{~m}, 2 \mathrm{H}), 5.71(\mathrm{~d}, J=8.0 \mathrm{~Hz}, 1 \mathrm{H}), 3.83(\mathrm{dd}, J=12.0,2.0 \mathrm{~Hz}, 1 \mathrm{H}), 3.70(\mathrm{dd}, J=12.0,4.4 \mathrm{~Hz}, 1 \mathrm{H}), 3.58-$ $3.43(\mathrm{~m}, 4 \mathrm{H}), 2.37$ (s, 3H); ${ }^{13} \mathbf{C}$ NMR (150 MHz, acetone- $\left.d_{6}\right) \delta: 164.2,138.1,135.0,133.0,131.8,131.4$, 129.9, 96.2, 78.6, 78.0, 73.9, 71.1, 62.5, 20.6; IR (KBr, cm $\left.{ }^{-1}\right): 3590,3331,3192,2877,2307,1754,1714$, 1642, 1479, 1399, 1292, 1244, 1197, 1109, 1025, 894, 810, 778, 639; HRMS-ESI ${ }^{-}$(m/z): Calcd. for $\mathrm{C}_{14} \mathrm{H}_{17} \mathrm{O}_{7} \mathrm{Cl}_{2}[\mathrm{M}+\mathrm{Cl}]^{-}$367.0333; found, 367.0346.

1-O-(2-Methylbenzoyl)- $\beta$-D-glucopyranoside (4i).<smiles>Cc1ccccc1C(=O)OC(O)[C@@H]1OC[C@@H](O)C(O)C1O</smiles>

General procedure A for the synthesis of glycoside was followed, starting with $\alpha$-D-glucose (540 mg, 3.0 mmol, 3.0 equiv.), 2-methylbenzoic acid (136 mg, $1.0 \mathrm{mmol}, 1.0$ equiv.), DIAD (394 $\mu \mathrm{L}, 2.0 \mathrm{mmol}, 2.0$ equiv.), and $\mathrm{Ph}_{3} \mathrm{P}$ (525 mg, $2.0 \mathrm{mmol}, 2.0$ equiv.) in 1,4-dioxane (100 mL) for $30 \mathrm{~min}$, affording the glucoside $(238 \mathrm{mg}, 79 \%, \alpha / \beta=1 / 99)$ as a white amorphous powder after purification by flash chromatography, eluting with $\mathrm{CHCl}_{3} / \mathrm{MeOH}(50: 1$ to $5: 1 \mathrm{v} / \mathrm{v})$. Analytical data: $[\alpha]_{\mathbf{D}}{ }^{21}=-16(c 0.5, \mathrm{MeOH})$; TLC $\left(\mathrm{CHCl}_{3} / \mathrm{MeOH} 5: 1 \mathrm{v} / \mathrm{v}\right): \mathrm{R}_{f}=0.28 ;{ }^{1} \mathbf{H}$ NMR $\left(400 \mathrm{MHz}\right.$, acetone- $\left.d_{6}+\mathrm{D}_{2} \mathrm{O}\right) \delta: 7.99(\mathrm{~d}, J=7.4 \mathrm{~Hz}, 1 \mathrm{H})$, $7.48(\mathrm{t}, J=6.8 \mathrm{~Hz}, 1 \mathrm{H}), 7.34-7.29(\mathrm{~m}, 2 \mathrm{H}), 5.71(\mathrm{~d}, J=8.2 \mathrm{~Hz}, 1 \mathrm{H}), 3.83(\mathrm{dd}, J=11.2,2.3 \mathrm{~Hz}, 1 \mathrm{H}), 3.68(\mathrm{dd}$, $J=12.4,4.6 \mathrm{~Hz}, 1 \mathrm{H}), 3.58-3.42(\mathrm{~m}, 4 \mathrm{H}), 2.55(\mathrm{~s}, 3 \mathrm{H}) ;{ }^{13} \mathbf{C}$ NMR $\left(100 \mathrm{MHz}\right.$, acetone- $\left.d_{6}+\mathrm{D}_{2} \mathrm{O}\right) \delta: 166.5$, 141.3, 133.4, 132.5, 131.6, 129.7, 126.7, 95.6, 78.4, 77.6, 73.6, 70.8, 62.1, 21.7; IR $\left(\mathrm{KBr}, \mathrm{cm}^{-1}\right): 3491,3355$, 2925, 1714, 1459, 1380, 1253, 1069, 738; HRMS-ESI ${ }^{+}(\mathrm{m} / \mathrm{z})$ : Calcd. for $\mathrm{C}_{14} \mathrm{H}_{18} \mathrm{O}_{7} \mathrm{Na}[\mathrm{M}+\mathrm{Na}]^{+} 321.0945$; found, 321.0955 . 
1-O-(Furan-3-carbonyl)- $\beta$-D-glucopyranoside (4j).

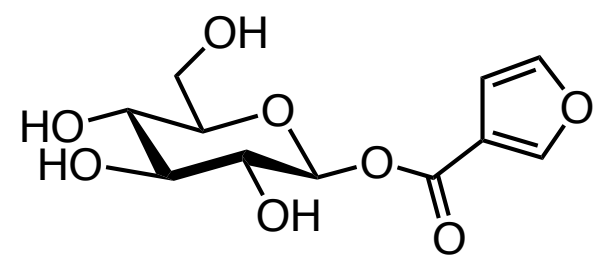

General procedure A for the synthesis of glycoside was followed, starting with $\alpha$-D-glucose (100 $\mathrm{mg}, 0.56$ mmol, 3.0 equiv.), 3-furoic acid (20.7 mg, 0.19 mmol, 1.0 equiv.), DIAD (72.9 $\mu \mathrm{L}, 0.37$ mmol, 2.0 equiv.), and $\mathrm{Ph}_{3} \mathrm{P}$ (97.0 mg, $0.37 \mathrm{mmol}, 2.0$ equiv.) in 1,4-dioxane $(19 \mathrm{~mL})$ for $30 \mathrm{~min}$, affording the glucoside (38.7 $\mathrm{mg}, 76 \%, \alpha / \beta=1 / 99)$ as a white amorphous powder after purification by flash chromatography, eluting with $\mathrm{CHCl}_{3} / \mathrm{MeOH}(50: 1$ to $5: 1 \mathrm{v} / \mathrm{v})$. Analytical data: $[\boldsymbol{\alpha}]_{\mathbf{D}}{ }^{\mathbf{2 0}}=-12(c 0.5, \mathrm{MeOH}) ; \mathbf{T L C}\left(\mathrm{CHCl}_{3} / \mathrm{MeOH} 5: 1 \mathrm{v} / \mathrm{v}\right)$ : $\mathrm{R}_{f}=0.17 ;{ }^{1} \mathrm{H}$ NMR $\left(400 \mathrm{MHz}, \mathrm{CD}_{3} \mathrm{CN}+\mathrm{D}_{2} \mathrm{O}\right) \delta: 8.35(\mathrm{~d}, J=1.2 \mathrm{~Hz} 1 \mathrm{H}), 7.70(\mathrm{t}, J=1.6 \mathrm{~Hz}, 1 \mathrm{H}), 6.92(\mathrm{~d}, J$ $=1.6 \mathrm{~Hz}, 1 \mathrm{H}), 5.69(\mathrm{~d}, J=7.6 \mathrm{~Hz}, 1 \mathrm{H}), 3.83(\mathrm{dd}, J=12.0,2.4 \mathrm{~Hz}, 1 \mathrm{H}), 3.69(\mathrm{dd}, J=12.4,5.6 \mathrm{~Hz}, 1 \mathrm{H})$, 3.57-3.38 (m, 4H); ${ }^{13}$ C NMR (100 MHz, acetone- $\left.d_{6}\right) \delta: 162.0,149.7,145.5,119.9,110.5,95.5,78.5,77.9$, 73.8, 71.1, 62.5; IR $\left(\mathrm{KBr}, \mathrm{cm}^{-1}\right): 3542,3435,2917,1722,1511,1308,1169,1077,1041,763,603$; HRMS-FAB $^{-}(\mathrm{m} / \mathrm{z})$ : Calcd. for $\mathrm{C}_{11} \mathrm{H}_{13} \mathrm{O}_{8}[\mathrm{M}-\mathrm{H}]^{-}$273.0610; found, 273.0607.

1-O-Cinnamoyl- $\beta$-D-glucopyranoside (4k).<smiles>O=C(/C=C/c1ccccc1)O[C@H](O)[C@@H](O)[C@H](O)CO</smiles>

General procedure A for the synthesis of glycoside was followed, starting with $\alpha$-D-glucose (100 mg, 0.56 mmol, 3.0 equiv.), trans-cinnamic acid (27.4 mg, 0.19 mmol, 1.0 equiv.), DIAD (72.9 $\mu \mathrm{L}, 0.37 \mathrm{mmol}, 2.0$ equiv.), and $\mathrm{Ph}_{3} \mathrm{P}$ (97.0 mg, $0.37 \mathrm{mmol}, 2.0$ equiv.) in 1,4-dioxane (19 mL) for $30 \mathrm{~min}$, affording the glucoside $(51.0 \mathrm{mg}, 86 \%, \alpha / \beta=1 / 99)$ as a white amorphous powder after purification by flash chromatography, eluting with $\mathrm{CHCl}_{3} / \mathrm{MeOH}(50: 1$ to $5: 1 \mathrm{v} / \mathrm{v})$. Analytical data: $[\boldsymbol{\alpha}]_{\mathbf{D}}{ }^{21}=+1.2$ (c 0.67 , acetone); TLC $\left(\mathrm{CHCl}_{3} / \mathrm{MeOH} 5: 1 \mathrm{v} / \mathrm{v}\right): \mathrm{R}_{f}=0.32 ;{ }^{1} \mathbf{H}$ NMR $\left(400 \mathrm{MHz}\right.$, acetone- $\left.d_{6}+\mathrm{D}_{2} \mathrm{O}\right) \delta: 7.78(\mathrm{~d}, J=$ $16.0 \mathrm{~Hz}, 1 \mathrm{H}), 7.73-7.70(\mathrm{~m}, 2 \mathrm{H}), 7.46-7.44(\mathrm{~m}, 3 \mathrm{H}), 6.58(\mathrm{~d}, J=16.0 \mathrm{~Hz}, 1 \mathrm{H}), 5.61(\mathrm{~d}, J=8.0 \mathrm{~Hz}, 1 \mathrm{H})$, 3.83-3.80 (m, $1 \mathrm{H}), 3.67(\mathrm{dd}, J=12.0,4.8 \mathrm{~Hz}, 1 \mathrm{H}), 3.53-3.40(\mathrm{~m}, 4 \mathrm{H}) ;{ }^{13} \mathbf{C}$ NMR (150 MHz, acetone- $\left.d_{6}\right) \delta$ : 165.8, 146.6, 135.3, 131.5, 129.9, 129.2, 118.6, 95.5, 78.5, 78.0, 73.9, 71.2, 62.5; IR (KBr, cm $\left.{ }^{-1}\right): 3491,3060$, 2933, 1702, 1634, 1582, 1455, 1376, 1340, 1281, 1133, 1077, 1013, 862, 763, 679; HRMS-FAB ${ }^{+}(\mathrm{m} / \mathrm{z})$ : Calcd. for $\mathrm{C}_{15} \mathrm{H}_{18} \mathrm{O}_{7} \mathrm{Na}[\mathrm{M}+\mathrm{Na}]^{+}$333.0950; found, 333.0955 . 
<smiles>O=C(CCc1nc(-c2ccccc2)c(-c2ccccc2)o1)O[C@H](O)[C@H](O)C(O)CO</smiles>

General procedure A for the synthesis of glycoside was followed, starting with $\alpha$-D-glucose (432 mg, 2.40 mmol, 3.0 equiv.), oxaprozin ( $235 \mathrm{mg}, 0.80 \mathrm{mmol}, 1.0$ equiv.), DIAD (315 $\mu \mathrm{L}, 1.6 \mathrm{mmol}, 2.0$ equiv.), and $\mathrm{Ph}_{3} \mathrm{P}$ (420 mg, $1.6 \mathrm{mmol}, 2.0$ equiv.) in 1,4-dioxane $(80 \mathrm{~mL}$ ) for $30 \mathrm{~min}$, affording the glucoside (257 $\mathrm{mg}$, $71 \%, \alpha / \beta=2 / 98)$ as a white amorphous powder after purification by flash chromatography, eluting with $\mathrm{CHCl}_{3} / \mathrm{MeOH}\left(50: 1\right.$ to 5:1 v/v). Analytical data: $[\alpha]_{\mathbf{D}}{ }^{20}=+4.0(c 1.0, \mathrm{MeOH}) ; \mathbf{T L C}\left(\mathrm{CHCl}_{3} / \mathrm{MeOH} 5: 1 \mathrm{v} / \mathrm{v}\right)$ : $\mathrm{R}_{f}=0.46 ;{ }^{1} \mathbf{H}$ NMR $\left(400 \mathrm{MHz}\right.$, acetone- $\left.d_{6}+\mathrm{D}_{2} \mathrm{O}\right) \delta: 7.65-7.58(\mathrm{~m}, 4 \mathrm{H}), 7.46-7.34(\mathrm{~m}, 6 \mathrm{H}), 5.55(\mathrm{~d}, J=8.4$ $\mathrm{Hz} 1 \mathrm{H}), 3.82-3.78(\mathrm{~m}, 1 \mathrm{H}), 3.69-3.65(\mathrm{~m}, 1 \mathrm{H}), 3.50-3.41$ (m, 2H), 3.36 (t, $J=8.4 \mathrm{~Hz}, 1 \mathrm{H}), 3.21-3.17$ (m, 1H), 3.04-2.95 (m, 4H); ${ }^{13}$ C NMR (100 MHz, acetone- $\left.d_{6}\right) \delta: 171.7,163.1,146.0,135.6,133.2,129.7,129.6$, 129.5, 129.3, 128.9, 128.5, 127.3, 95.5, 78.3, 77.3, 73.4, 70.6, 62.0, 31.2, 23.5; IR (KBr, cm $\left.{ }^{-1}\right): 3367,1753$, 1570, 1443, 1363, 1328, 1173, 1077, 1029, 763, 690; HRMS-FAB ${ }^{+}(\mathrm{m} / \mathrm{z})$ : Calcd. for $\mathrm{C}_{24} \mathrm{H}_{25} \mathrm{NO}_{8} \mathrm{Na}[\mathrm{M}]^{+}$ 478.1478; found, 478.1478 .

1-O-\{(2S)-2-(6-Methoxynaphthalen-2-yl)propanoyl)- $\beta$-D-glucopyranoside (4m).

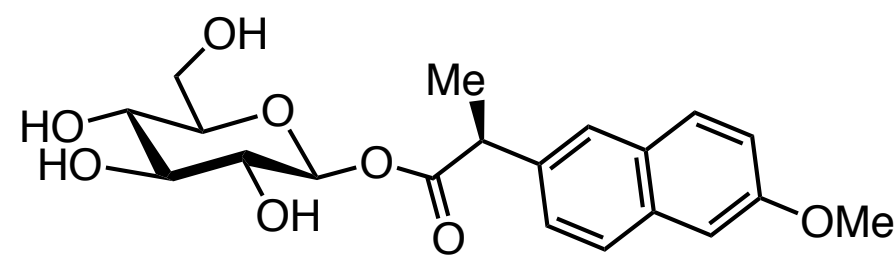

General procedure A for the synthesis of glycoside was followed, starting with $\alpha$-D-glucose (1.00 g, 5.6 mmol, 3.0 equiv.), naproxen (426 mg, $1.9 \mathrm{mmol}, 1.0$ equiv.), DIAD (728 $\mu \mathrm{L}, 3.7 \mathrm{mmol}, 2.0$ equiv.), and $\mathrm{Ph}_{3} \mathrm{P}$ (970 mg, $3.7 \mathrm{mmol}, 2.0$ equiv.) in 1,4-dioxane (185 mL) for $1 \mathrm{~h}$, affording the glucoside (452 mg, 62\%, $\alpha / \beta=1 / 99)$ as a white amorphous powder after purification by flash chromatography, eluting with $\mathrm{CHCl}_{3} / \mathrm{MeOH}\left(50: 1\right.$ to 5:1 v/v). Analytical data: $[\boldsymbol{\alpha}]_{\mathbf{D}}{ }^{\mathbf{2 0}}=+21(c 1.0, \mathrm{MeOH}) ; \mathbf{T L C}\left(\mathrm{CHCl}_{3} / \mathrm{MeOH} 5: 1 \mathrm{v} / \mathrm{v}\right)$ : $\mathrm{R}_{f}=0.38 ;{ }^{1} \mathbf{H}$ NMR $\left(400 \mathrm{MHz}\right.$, acetone- $\left.d_{6}+\mathrm{D}_{2} \mathrm{O}\right) \delta: 7.79-7.76(\mathrm{~m}, 3 \mathrm{H}), 7.45(\mathrm{dd}, J=8.0,1.2 \mathrm{~Hz}, 1 \mathrm{H}), 7.27$ $(\mathrm{d}, J=2.4 \mathrm{~Hz}, 1 \mathrm{H}), 7.13$ (dd, $J=8.8,2.8 \mathrm{~Hz}, 2 \mathrm{H}), 5.52$ (d, $J=7.6 \mathrm{~Hz}, 1 \mathrm{H}), 3.97$ (q, $J=7.2 \mathrm{~Hz}, 1 \mathrm{H}), 3.89$ (s, $3 \mathrm{H}), 3.73(\mathrm{dd}, J=11.6,2.0 \mathrm{~Hz}, 1 \mathrm{H}), 3.58$ (dd, $J=12.0,5.2 \mathrm{~Hz}, 1 \mathrm{H}), 3.49-3.29$ (m, 4H), 1.52 (d, $J=7.2 \mathrm{~Hz}$, $3 \mathrm{H}) ;{ }^{13} \mathrm{C}$ NMR (100 MHz, acetone- $\left.d_{6}\right) \delta: 174.0,158.6,136.5,134.7,130.1,129.8,128.0,127.2,126.9$, 119.7, 106.4, 95.6, 78.4, 77.6, 73.5, 70.8, 62.1, 55.6, 45.8, 19.4; IR (KBr, cm $\left.{ }^{-1}\right): 3390,3191,2976,1742$, 1626, 1606, 1268, 1225, 1189, 1101, 1065, 1025, 858, 822; HRMS-FAB ${ }^{+}(\mathrm{m} / \mathrm{z})$ : Calcd. for $\mathrm{C}_{20} \mathrm{H}_{24} \mathrm{O}_{8}[\mathrm{M}]^{+}$ 392.1471; found, 392.1468 . 
1-O-\{5-(2,5-Dimethylphenoxy)2,2-dimethylpentanoyl)- $\beta$-D-glucopyranoside (4n).<smiles>Cc1ccc(C)c(OCCCC(C)(C)C(=O)OC2OC(CO)[C@@H](O)C(O)[C@H]2O)c1</smiles>

Modified general procedure A for the synthesis of glycoside was followed, starting with $\alpha$-D-glucose (200 mg, $1.1 \mathrm{mmol}, 3.0$ equiv.), gemfibrozil (92.6 mg, $0.37 \mathrm{mmol}, 1.0$ equiv.), DIAD (291 $\mu \mathrm{L}, 1.5 \mathrm{mmol}, 4.0$ equiv.), and $\mathrm{Ph}_{3} \mathrm{P}$ (388 mg, $1.5 \mathrm{mmol}, 4.0$ equiv.) in 1,4-dioxane (37 mL) for $1 \mathrm{~h}$, affording the glucoside (74.0 mg, $48 \%, \alpha / \beta=1 / 99$ ) as a white amorphous powder after purification by flash chromatography, eluting with $\mathrm{CHCl}_{3} / \mathrm{MeOH}(50: 1$ to $5: 1 \mathrm{v} / \mathrm{v})$. Analytical data: $[\alpha]_{\mathbf{D}}{ }^{20}=-0.2(c 1.0, \mathrm{MeOH}) ; \mathbf{T L C}\left(\mathrm{CHCl}_{3} / \mathrm{MeOH} 5: 1\right.$ $\mathrm{v} / \mathrm{v}): \mathrm{R}_{f}=0.54 ;{ }^{1} \mathbf{H}$ NMR (400 MHz, acetone- $\left.d_{6}+\mathrm{D}_{2} \mathrm{O}\right) \delta: 6.96(\mathrm{~d}, J=7.2 \mathrm{~Hz}, 1 \mathrm{H}), 6.70(\mathrm{br} \mathrm{s}, 1 \mathrm{H}), 6.62(\mathrm{~d}, J$ $=7.2 \mathrm{~Hz}, 1 \mathrm{H}), 5.50(\mathrm{~d}, J=8.0 \mathrm{~Hz}, 1 \mathrm{H}), 3.95-3.93(\mathrm{~m}, 2 \mathrm{H}), 3.79(\mathrm{dd}, J=11.6,2.0 \mathrm{~Hz}, 1 \mathrm{H}), 3.68-3.64(\mathrm{~m}$, 1H), 3.47-3.29 (m, 4H), 2.25 (s, 3H), 2.12 (s, 3H), 1.79-1.76 (m, 2H), $1.23(\mathrm{~s}, 3 \mathrm{H}), 1.22(\mathrm{~s}, 3 \mathrm{H}) ;{ }^{13} \mathbf{C}$ NMR $\left(100 \mathrm{MHz}\right.$, acetone- $\left.d_{6}\right) \delta: 177.2,157.5,137.0,130.7,123.5,121.2,112.6,95.0,77.9,77.0,73.0,70.3,68.4$, 61.7, 42.5, 37.3, 25.3, 25.1, 24.9, 21.2, 15.8; IR (KBr, cm $\left.{ }^{-1}\right): 3386,2933,1734,1615,1510,1471,1419$, 1387, 1268, 1120, 1073, 806; HRMS-ESI $^{-}(\mathrm{m} / \mathrm{z})$ : Calcd. for $\mathrm{C}_{21} \mathrm{H}_{32} \mathrm{O}_{8} \mathrm{Cl}[\mathrm{M}+\mathrm{Cl}]^{-}$447.1777; found, 447.1780 .

1-O-[4-\{4-Bis(2-chloroethyl)aminophenyl\}butanoyl]- $\beta$-D-glucopyranoside (40).<smiles>O=C(CCCc1ccc(N(CCCl)CCCl)cc1)O[C@H]1O[C@H](CO)[C@@H](O)[C@H](O)[C@H]1O</smiles>

General procedure A for the synthesis of glycoside was followed, starting with $\alpha$-D-glucose (711 $\mathrm{mg}, 4.0$ mmol, 3.0 equiv.), chlorambucil (400 mg, $1.3 \mathrm{mmol}, 1.0$ equiv.), DIAD (518 $\mu \mathrm{L}, 2.6 \mathrm{mmol}, 2.0$ equiv.), and $\mathrm{Ph}_{3} \mathrm{P}$ (690 mg, $2.6 \mathrm{mmol}, 2.0$ equiv.) in 1,4-dioxane (132 mL) for $30 \mathrm{~min}$, affording the glucoside (405 mg, $66 \%, \alpha / \beta=1 / 99)$ as a white amorphous powder after purification by flash chromatography, eluting with $\mathrm{CHCl}_{3} / \mathrm{MeOH}(50: 1$ to $5: 1 \mathrm{v} / \mathrm{v})$. Analytical data: $[\alpha]_{\mathbf{D}}{ }^{21}=+3.2$ (c 1.14, acetone); TLC $\left(\mathrm{CHCl}_{3} / \mathrm{MeOH} 5: 1\right.$ $\mathrm{v} / \mathrm{v}): \mathrm{R}_{f}=0.48 ;{ }^{1} \mathbf{H}$ NMR (400 MHz, acetone- $\left.d_{6}+\mathrm{D}_{2} \mathrm{O}\right) \delta: 7.09(\mathrm{~d}, J=8.8 \mathrm{~Hz}, 2 \mathrm{H}), 6.73(\mathrm{~d}, J=6.4 \mathrm{~Hz}, 2 \mathrm{H})$, $5.50(\mathrm{~d}, J=8.0 \mathrm{~Hz}, 1 \mathrm{H}), 3.80-3.70(\mathrm{~m}, 9 \mathrm{H}), 3.67-3.63(\mathrm{~m}, 1 \mathrm{H}), 3.49-3.38(\mathrm{~m}, 3 \mathrm{H}), 3.32(\mathrm{t}, J=8.4 \mathrm{~Hz}, 1 \mathrm{H})$, $2.56(\mathrm{t}, J=7.6 \mathrm{~Hz}, 2 \mathrm{H}), 2.39-2.34(\mathrm{~m}, 2 \mathrm{H}), 1.86$ (quint, $J=7.2 \mathrm{~Hz}, 2 \mathrm{H}) ;{ }^{13} \mathbf{C}$ NMR (100 MHz, acetone- $d_{6}$ ) $\delta: 172.8,145.6,131.0,130.4,113.0,95.1,78.3,77.6,73.5,70.8,62.2,53.8,41.6,34.4,33.9,27.4$; IR $(\mathrm{KBr}$, $\left.\mathrm{cm}^{-1}\right): 3550,3307,2925,1714,1594,1451,1395,1316,1281,1077,1021,711$; HRMS-ESI ${ }^{-}(\mathrm{m} / z):$ Calcd. for $\mathrm{C}_{20} \mathrm{H}_{29} \mathrm{NO}_{7} \mathrm{Cl}_{2}[\mathrm{M}+\mathrm{Cl}]^{-}$500.1005; found, 500.1015 . 


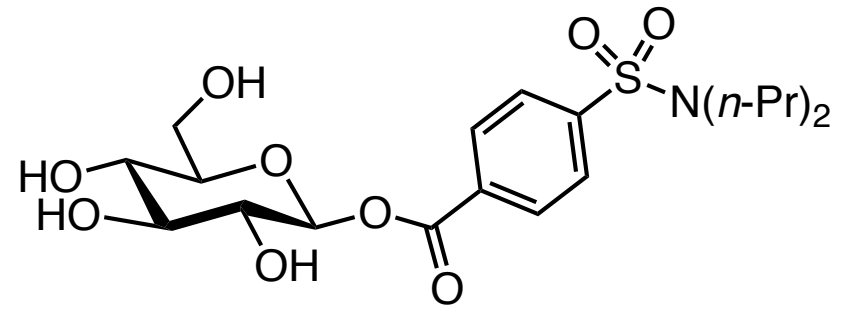

General procedure A for the synthesis of glycoside was followed, starting with $\alpha$-D-glucose (200 mg, 1.1 mmol, 3.0 equiv.), probenecid (105 mg, 0.37 mmol, 1.0 equiv.), DIAD (146 $\mu \mathrm{L}, 0.74 \mathrm{mmol}, 2.0$ equiv.), and $\mathrm{Ph}_{3} \mathrm{P}$ (194 mg, $0.74 \mathrm{mmol}, 2.0$ equiv.) in 1,4-dioxane (37 mL) for $30 \mathrm{~min}$, affording the glucoside (107 mg, $65 \%, \alpha / \beta=2 / 98)$ as a white amorphous powder after purification by flash chromatography, eluting with $\mathrm{CHCl}_{3} / \mathrm{MeOH}\left(50: 1\right.$ to 5:1 v/v). Analytical data: $[\alpha]_{\mathbf{D}}{ }^{20}=-6.0(c 1.0, \mathrm{MeOH}) ; \mathbf{T L C}\left(\mathrm{CHCl}_{3} / \mathrm{MeOH} 5: 1 \mathrm{v} / \mathrm{v}\right)$ : $\mathrm{R}_{f}=0.38 ;{ }^{1} \mathbf{H}$ NMR $\left(600 \mathrm{MHz}\right.$, acetone- $\left.d_{6}+\mathrm{D}_{2} \mathrm{O}\right) \delta: 8.27(\mathrm{~d}, J=9.0 \mathrm{~Hz}, 2 \mathrm{H}), 7.99(\mathrm{~d}, J=9.0 \mathrm{~Hz}, 2 \mathrm{H}), 5.76$ $(\mathrm{d}, J=8.4 \mathrm{~Hz}, 1 \mathrm{H}), 3.83(\mathrm{dd}, J=12.0,3.0 \mathrm{~Hz}, 1 \mathrm{H}), 3.69(\mathrm{dd}, J=12.0,4.8 \mathrm{~Hz}, 1 \mathrm{H}), 3.56-3.45(\mathrm{~m}, 4 \mathrm{H}), 3.14$ $(\mathrm{t}, J=7.8 \mathrm{~Hz}, 4 \mathrm{H}), 1.55$ (sext, $J=7.8 \mathrm{~Hz}, 4 \mathrm{H}), 0.85(\mathrm{t}, J=7.2 \mathrm{~Hz}, 6 \mathrm{H}) ;{ }^{13} \mathbf{C}$ NMR $\left(150 \mathrm{MHz}\right.$, acetone- $\left.d_{6}\right) \delta$ : 164.7, 145.7, 133.8, 131.4, 128.1, 96.4, 78.6, 77.6, 73.7, 70.9, 62.2, 50.7, 22.6, 11.3; IR (KBr, cm $\left.{ }^{-1}\right): 3406$, 2933, 2877, 1742, 1464, 1404, 1335, 1268, 1157, 1085, 989, 862, 750, 611; HRMS-FAB $^{+}(\mathrm{m} / \mathrm{z})$ : Calcd. for $\mathrm{C}_{19} \mathrm{H}_{29} \mathrm{NO}_{9} \mathrm{SNa}[\mathrm{M}]^{+}$470.1461; found, 470.1464 .

\section{1-O-(2-Allyl-4-nitrophenyl)- $\beta$-D-glucopyranoside (4q).}

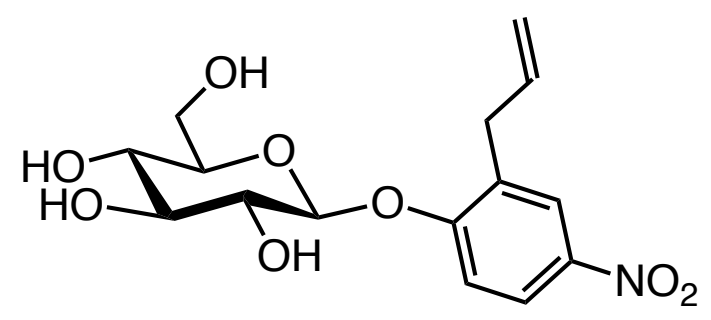

General procedure A for the synthesis of glycoside was followed, starting with $\alpha$-D-glucose (108 $\mathrm{mg}, 0.60$ mmol, 3.0 equiv.), 2-allyl-4-nitrophenol ${ }^{3}$ (35.8 mg, 0.20 mmol, 1.0 equiv.), DIAD (78.8 $\mu \mathrm{L}, 0.40 \mathrm{mmol}, 2.0$ equiv.), $\mathrm{Ph}_{3} \mathrm{P}$ (105 mg, $0.40 \mathrm{mmol}, 2.0$ equiv.) in 1,4-dioxane (20 mL) for $30 \mathrm{~min}$, affording the glucoside (43.7 $\mathrm{mg}, 64 \%, \alpha / \beta=1 / 99$ ) as a pale yellow amorphous powder after purification by flash chromatography, eluting with $\mathrm{CHCl}_{3} / \mathrm{MeOH}(50: 1$ to $5: 1 \mathrm{v} / \mathrm{v})$. Analytical data: $[\boldsymbol{\alpha}]_{\mathbf{D}}{ }^{\mathbf{2 1}}=-76$ (c 1.0, MeOH); TLC $\left(\mathrm{CHCl}_{3} / \mathrm{MeOH} 5: 1 \mathrm{v} / \mathrm{v}\right): \mathrm{R}_{f}=0.44 ;{ }^{1} \mathbf{H}$ NMR $\left(400 \mathrm{MHz}\right.$, acetone- $\left.d_{6}+\mathrm{D}_{2} \mathrm{O}\right) \delta: 8.09(\mathrm{dd}, J=9.2,2.7 \mathrm{~Hz}, 1 \mathrm{H})$, $8.04(\mathrm{~d}, J=2.8 \mathrm{~Hz}, 1 \mathrm{H}), 7.37$ (d, $J=9.2 \mathrm{~Hz}, 1 \mathrm{H}), 6.05$ (ddt, $J=18.6,10.1,6.9 \mathrm{~Hz}, 1 \mathrm{H}), 5.21-5.08(\mathrm{~m}, 3 \mathrm{H})$, 3.89 (dd, $J=12.0,2.8 \mathrm{~Hz}, 1 \mathrm{H}), 3.67(\mathrm{dd}, J=12.0,4.4 \mathrm{~Hz}, 1 \mathrm{H}), 3.62-3.42(\mathrm{~m}, 6 \mathrm{H}) ;{ }^{13} \mathrm{C}$ NMR $(100 \mathrm{MHz}$, acetone- $\left.d_{6}+\mathrm{D}_{2} \mathrm{O}\right) \delta: 161.1,142.9,136.5,131.5,125.5,124.4,117.2,115.5,101.5,77.9,77.5,74.3,70.8,62.2$, 34.5; IR (KBr, cm $\left.{ }^{-1}\right): 3279,2937,1591,1515,1358,1243$, 1972, 1044, 673; HRMS-ESI ${ }^{-}$( $\left.\mathrm{m} / \mathrm{z}\right)$ : Calcd. for $\mathrm{C}_{15} \mathrm{H}_{19} \mathrm{NO}_{8} \mathrm{Cl}[\mathrm{M}+\mathrm{Cl}]^{-}$376.0805; found, 376.0814. 
1-O-(2-Bromo-4-formylphenyl)- $\beta$-D-glucopyranoside (4r).

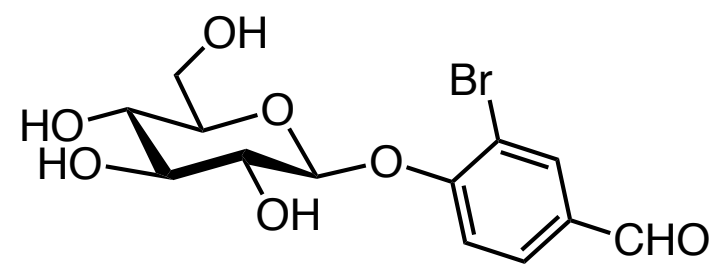

General procedure A for the synthesis of glycoside was followed, starting with $\alpha$-D-glucose (108 mg, 0.60 mmol, 3.0 equiv.), 3-bromo-4-hydroxybenzaldehyde ${ }^{4}$ (40.2 mg, $0.20 \mathrm{mmol}, 1.0$ equiv.), DIAD (78.8 $\mu \mathrm{L}$, 0.40 mmol, 2.0 equiv.), $\mathrm{Ph}_{3} \mathrm{P}$ (105 mg, $0.40 \mathrm{mmol}, 2.0$ equiv.) in 1,4-dioxane ( $20 \mathrm{~mL}$ ) for $30 \mathrm{~min}$, affording the glucoside (26.9 $\mathrm{mg}, 37 \%, \alpha / \beta=2 / 98)$ as a white amorphous powder after purification by flash chromatography, eluting with $\mathrm{CHCl}_{3} / \mathrm{MeOH}(50: 1$ to $5: 1 \mathrm{v} / \mathrm{v})$. Analytical data: $[\alpha]_{\mathbf{D}}{ }^{21}=-63(c 1.0, \mathrm{MeOH})$; TLC $\left(\mathrm{CHCl}_{3} / \mathrm{MeOH} 5: 1 \mathrm{v} / \mathrm{v}\right): \mathrm{R}_{f}=0.34 ;{ }^{1} \mathbf{H}$ NMR $\left(400 \mathrm{MHz}\right.$, acetone- $\left.d_{6}+\mathrm{D}_{2} \mathrm{O}\right) \delta: 9.90(\mathrm{~s}, 1 \mathrm{H}), 8.10(\mathrm{~d}, J=$ $1.8 \mathrm{~Hz}, 1 \mathrm{H}), 7.89$ (dd, $J=8.7,2.1 \mathrm{~Hz}, 1 \mathrm{H}), 7.45(\mathrm{~d}, J=8.7 \mathrm{~Hz}, 1 \mathrm{H}), 5.25(\mathrm{~d}, J=7.3 \mathrm{~Hz}, 1 \mathrm{H}), 3.87$ (dd, $J=$ 11.7, $2.5 \mathrm{~Hz}, 1 \mathrm{H}), 3.68(\mathrm{dd}, J=11.9,5.5 \mathrm{~Hz}, 1 \mathrm{H}), 3.63-3.43(\mathrm{~m}, 4 \mathrm{H}) ;{ }^{13} \mathbf{C}$ NMR (100 MHz, acetone- $\left.d_{6}+\mathrm{D}_{2} \mathrm{O}\right) \delta: 190.7,159.4,134.9,132.8,131.6,116.8,113.2,101.3,78.0,77.7,74.2,70.7,62.2$; IR $\left(\mathrm{KBr}, \mathrm{cm}^{-1}\right): 3414,2889,1674,1593,1494,1264,1080,1053,881,818,657,630$; HRMS-ESI ${ }^{-}(\mathrm{m} / z)$ : Calcd. for $\mathrm{C}_{13} \mathrm{H}_{14} \mathrm{BrO}_{7}[\mathrm{M}-\mathrm{H}]^{-}$360.9928; found, 360.9917 .

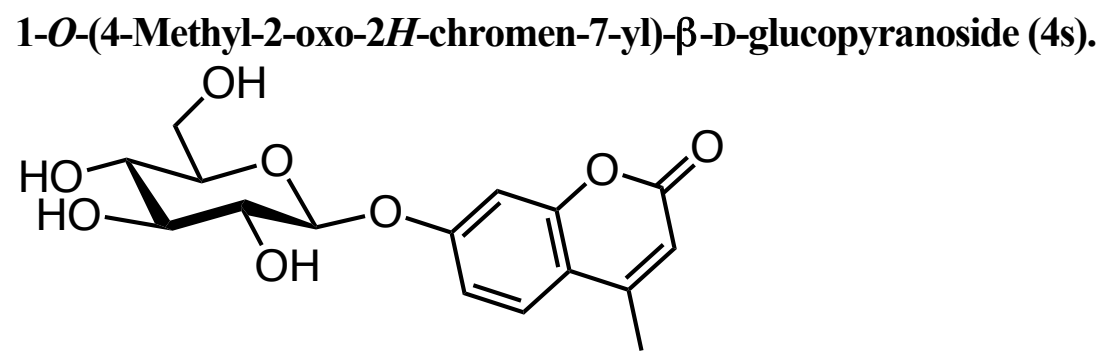

A round-bottom flask was charged with $\alpha$-D-glucose $(216 \mathrm{mg}, 1.2 \mathrm{mmol}, 3.0$ equiv.) and dehydrated 1,4-dioxane $(40 \mathrm{~mL})$ under an $\mathrm{Ar}$ atmosphere. After ultrasound irradiation of the suspension of $\alpha$-D-glucose in 1,4-dioxane for $15 \mathrm{~min}$, the suspension was warmed to $55^{\circ} \mathrm{C}$, using oil bath, and 4-methylumbelliferone (70.5 mg, $0.40 \mathrm{mmol}, 1.0$ equiv.) and $\mathrm{Ph}_{3} \mathrm{P}(210 \mathrm{mg}, 0.80 \mathrm{mmol}, 2.0$ equiv.) were added. DIAD (158 $\mu \mathrm{L}$, $0.80 \mathrm{mmol}, 2.0$ equiv.) was added dropwise by syringe and the reaction mixture was stirred at $55^{\circ} \mathrm{C}$. After stirring vigorously for $15 \mathrm{~min}, \mathrm{Ph}_{3} \mathrm{P}(210 \mathrm{mg}, 0.80 \mathrm{mmol}, 2.0$ equiv.) and DIAD (158 $\mu \mathrm{L}, 0.80 \mathrm{mmol}, 2.0$ equiv.) were added again in the same manner and stirred at $55^{\circ} \mathrm{C}$, using oil bath, for $45 \mathrm{~min}$. The reaction mixture was quenched with $\mathrm{MeOH}$, stirred for $5 \mathrm{~min}$, and concentrated in vacuo at $40{ }^{\circ} \mathrm{C}$ (water bath) to give a residue. The residue was directly purified by flash column chromatography $\left(\mathrm{SiO}_{2}, \mathrm{CHCl}_{3} / \mathrm{MeOH} 50: 1\right.$ to $5: 1 \mathrm{v} / \mathrm{v})$ to give the glucoside $(111 \mathrm{mg}, 82 \%, \alpha / \beta=3 / 97)$ as a white amorphous powder. Analytical data: $[\boldsymbol{\alpha}]_{\mathbf{D}}{ }^{\mathbf{2 0}}=-92$ (c $\left.0.5, \mathrm{MeOH}\right) ; \mathbf{T L C}\left(\mathrm{CHCl}_{3} / \mathrm{MeOH} 5: 1 \mathrm{v} / \mathrm{v}\right): \mathrm{R}_{f}=0.28 ;{ }^{1} \mathbf{H}$ NMR (400 MHz, acetone- $\left.d_{6}+\mathrm{D}_{2} \mathrm{O}\right) \delta: 7.69(\mathrm{~d}, J=8.7 \mathrm{~Hz}, 1 \mathrm{H}), 7.06(\mathrm{dd}, J=8.7,2.3 \mathrm{~Hz}, 1 \mathrm{H}), 7.01(\mathrm{~d}, J=2.8 \mathrm{~Hz}, 1 \mathrm{H}), 6.17(\mathrm{~s}$, $1 \mathrm{H}), 5.10$ (d, $J=7.3 \mathrm{~Hz}, 1 \mathrm{H}), 3.89$ (dd, $J=11.9,2.3 \mathrm{~Hz}, 1 \mathrm{H}), 3.68$ (dd, $J=11.9,5.5 \mathrm{~Hz}, 1 \mathrm{H}), 3.64-3.42$ (m, 4H), 2.44 (s, 3H); ${ }^{13} \mathbf{C}$ NMR (100 MHz, acetone- $\left.d_{6}+\mathrm{D}_{2} \mathrm{O}\right) \delta: 161.4,161.2,155.7,153.9,127.0,115.4,114.3$, 112.8, 104.4, 101.5, 77.8, 77.5, 74.3, 70.9, 62.2, 18.5; IR $\left(\mathrm{KBr}, \mathrm{cm}^{-1}\right): 3407,2897,1713,1620,1393,1296$, 
1089, 1038, 855, 644; HRMS-ESI $^{-}(\mathrm{m} / \mathrm{z})$ : Calcd. for $\mathrm{C}_{16} \mathrm{H}_{18} \mathrm{O}_{8} \mathrm{Cl}$ [M+Cl$]^{-}$373.0696; found, 373.0692 .

tert-Butyl ( $\beta$-D-glucopyranosyl)\{(2-nitrophenyl)sulfonyl $\}$ carbamate (4t).

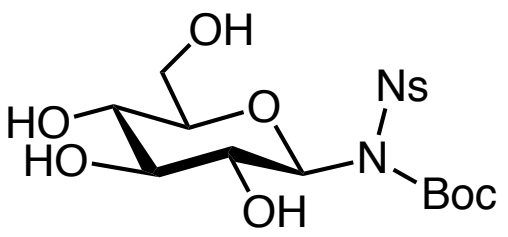

Modified general procedure A was followed, starting with $\alpha$-D-glucose (1.79 g, 9.9 mmol, 3.0 equiv.), $\mathrm{NsNHBoc}^{4}$ (1.0 g, 3.3 mmol, 1.0 equiv.), DIAD (2.6 mL, 13 mmol, 2.0 equiv.), $\mathrm{Ph}_{3} \mathrm{P}$ (3.5 g, 13 mmol, 2.0 equiv.) in 1,4-dioxane ( $330 \mathrm{~mL}$ ) at rt for $5 \mathrm{~min}$, adding extra DIAD (2.6 mL, $13 \mathrm{mmol}, 2.0$ equiv.) and $\mathrm{Ph}_{3} \mathrm{P}$ ( $3.5 \mathrm{~g}, 13 \mathrm{mmol}, 2.0$ equiv.) at $\mathrm{rt}$ for $25 \mathrm{~min}$, affording the glucoside $(631 \mathrm{mg}, 41 \%, \alpha / \beta=1 / 99)$ as a white amorphous powder after purification by two-time flash chromatography, eluting with $\left(1^{\text {st }}\right) \mathrm{CHCl}_{3} / \mathrm{MeOH}$ $(50: 1$ to $5: 1 \mathrm{v} / \mathrm{v})$ and then $\left(2^{\text {nd }}\right) \mathrm{CH}_{3} \mathrm{CN} / \mathrm{CHCl}_{3}(2: 1 \mathrm{v} / \mathrm{v})$. Analytical data: $[\alpha]_{\mathbf{D}}{ }^{21}=+321(c 0.55$, acetone $)$; TLC $\left(\mathrm{CHCl}_{3} / \mathrm{MeOH} 5: 1 \mathrm{v} / \mathrm{v}\right): \mathrm{R}_{f}=0.33 ;{ }^{1} \mathbf{H}$ NMR $\left(400 \mathrm{MHz}\right.$, acetone- $\left.d_{6}+\mathrm{D}_{2} \mathrm{O}\right) \delta: 8.62(\mathrm{~d}, J=7.6 \mathrm{~Hz}, 1 \mathrm{H})$, 7.95-7.84 (m, 3H), 5.28 (d, $J=9.2 \mathrm{~Hz}, 1 \mathrm{H}), 4.35$ (t, $J=9.2 \mathrm{~Hz}, 1 \mathrm{H}), 3.92$ (dd, $J=12.0,2.4 \mathrm{~Hz}, 1 \mathrm{H}), 3.70$ $(\mathrm{dd}, J=12.0,6.0 \mathrm{~Hz}, 1 \mathrm{H}), 3.52-3.38(\mathrm{~m}, 3 \mathrm{H}), 1.23(\mathrm{~s}, 9 \mathrm{H}) ;{ }^{13} \mathbf{C}$ NMR (100 MHz, acetone- $\left.d_{6}\right) \delta: 149.9,149.3$, 134.95, 134.90, 133.3, 130.7, 125.1, 87.6, 85.6, 80.7, 79.5, 71.4, 71.2, 62.7, 27.7; IR $\left(\mathrm{KBr}, \mathrm{cm}^{-1}\right): 3395,2980$, 1742, 1705, 1543, 1455, 1372, 1284, 1260, 1180, 1145, 1077, 742; HRMS-ESI ${ }^{+}(\mathrm{m} / \mathrm{z})$ : Calcd. for $\mathrm{C}_{17} \mathrm{H}_{24} \mathrm{~N}_{2} \mathrm{O}_{11} \mathrm{SNa}[\mathrm{M}+\mathrm{Na}]^{+}$487.0993; found, 487.1001 .

$N$-( $\beta$-D-Glucopyranosyl)-4,5,6,7-tetrachloro-2-isoindoline-1,3-dione (4u).<smiles>O=C1c2c(Cl)c(Cl)c(Cl)c(Cl)c2C(=O)N1C1OC(CO)[C@@H](O)[C@H](O)[C@H]1O</smiles>

General procedure A for the synthesis of glycoside was followed, starting with $\alpha$-D-glucose (200 mg, 1.1 mmol, 3.0 equiv.), 3,4,5,6-tetrachlorophthalimide (105 mg, $0.37 \mathrm{mmol}, 1.0$ equiv.), DIAD (146 $\mu \mathrm{L}, 0.74$ mmol, 2.0 equiv.), $\mathrm{Ph}_{3} \mathrm{P}$ (194 mg, $0.74 \mathrm{mmol}, 2.0$ equiv.) in 1,4-dioxane ( $37 \mathrm{~mL}$ ) for $30 \mathrm{~min}$, affording the glucoside (117 mg, 71\%, $\alpha / \beta=1 / 99)$ as a white amorphous powder after purification by flash chromatography, eluting with $\mathrm{CHCl}_{3} / \mathrm{MeOH}(50: 1$ to $5: 1 \mathrm{v} / \mathrm{v})$. Analytical data: $[\boldsymbol{\alpha}]_{\mathbf{D}}{ }^{21}=-3.1$ (c 0.74 , acetone); TLC $\left(\mathrm{CHCl}_{3} / \mathrm{MeOH} 5: 1 \mathrm{v} / \mathrm{v}\right): \mathrm{R}_{f}=0.40 ;{ }^{1} \mathbf{H}$ NMR $\left(400 \mathrm{MHz}\right.$, acetone- $\left.d_{6}+\mathrm{D}_{2} \mathrm{O}\right) \delta: 5.16(\mathrm{~d}, J=9.6$ $\mathrm{Hz}, 1 \mathrm{H}), 4.38(\mathrm{t}, J=9.2 \mathrm{~Hz}, 1 \mathrm{H}), 3.85(\mathrm{dd}, J=12.0,2.0 \mathrm{~Hz}, 1 \mathrm{H}), 3.65(\mathrm{dd}, J=12.4,5.2 \mathrm{~Hz}, 1 \mathrm{H}), 3.58-3.45$ $(\mathrm{m}, 3 \mathrm{H}) ;{ }^{13} \mathbf{C}$ NMR (100 MHz, acetone- $\left.d_{6}\right) \delta: 163.3,140.3,130.2,128.7,81.8,80.6,78.9,71.2,69.6,62.7$; IR $\left(\mathrm{KBr}, \mathrm{cm}^{-1}\right)$ : 3323, 2980, 1790, 1726, 1388, 1372, 1145, 1077, 1045, 905, 742; HRMS-ESI ${ }^{-}(\mathrm{m} / \mathrm{z})$ : Calcd. for $\mathrm{C}_{14} \mathrm{H}_{11} \mathrm{NO}_{7} \mathrm{Cl}_{5}[\mathrm{M}+\mathrm{Cl}]^{-}$479.8966; found, 479.8984 . 
Thotneoside C (5).

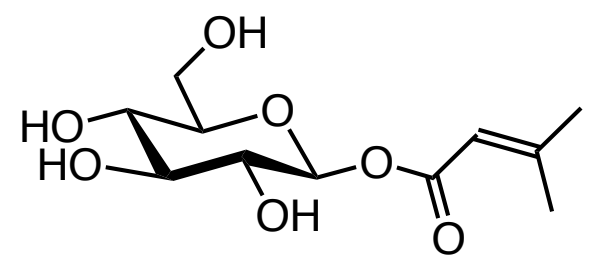

General procedure A for the synthesis of glycoside was followed, starting with $\alpha$-D-glucose $(270 \mathrm{mg}, 1.5$ mmol, 3.0 equiv.), 3-phenylpropanoic acid (50.1 mg, 0.50 mmol, 1.0 equiv.), DIAD (197 $\mu \mathrm{L}, 1.0 \mathrm{mmol}, 2.0$ equiv.), and $\mathrm{Ph}_{3} \mathrm{P}$ (262 mg, $1.0 \mathrm{mmol}, 2.0$ equiv.) in 1,4-dioxane (50 mL) for $30 \mathrm{~min}$, affording thotneoside $\mathrm{C} \mathrm{(5)} \mathrm{(88.4} \mathrm{mg,} 67 \%, \alpha / \beta=2 / 98)$ as a white amorphous powder after purification by flash chromatography, eluting with $\mathrm{CHCl}_{3} / \mathrm{MeOH}(50: 1$ to $5: 1 \mathrm{v} / \mathrm{v})$. Analytical data: $[\alpha]_{\mathbf{D}}{ }^{21}=-36$ (c 1.0, MeOH); TLC $\left(\mathrm{CHCl}_{3} / \mathrm{MeOH} 5: 1 \mathrm{v} / \mathrm{v}\right): \mathrm{R}_{f}=0.35 ;{ }^{1} \mathbf{H}$ NMR $\left(400 \mathrm{MHz}\right.$, acetone- $\left.d_{6}+\mathrm{D}_{2} \mathrm{O}\right) \delta: 5.71-5.69(\mathrm{~m}, 1 \mathrm{H}), 5.51(\mathrm{~d}, J=$ $8.2 \mathrm{~Hz}, 1 \mathrm{H}), 3.79$ (dd, $J=11.9,1.8 \mathrm{~Hz}, 1 \mathrm{H}), 3.68-3.62(\mathrm{~m}, 1 \mathrm{H}), 3.49-3.31$ (m, 4H), 2.16 (s, 3H), 1.93 (s, $3 \mathrm{H}) ;{ }^{13} \mathbf{C}$ NMR $\left(100 \mathrm{MHz}\right.$, acetone- $\left.d_{6}+\mathrm{D}_{2} \mathrm{O}\right) \delta: 165.2,159.8,116.2,94.6,78.3,78.0,73.8,71.1,62.4,27.3$, 20.3; IR (KBr, cm $\left.{ }^{-1}\right): 3364,2928,1739,1646,1454,1382,1227,1142,1073,847$; HRMS-ESI ${ }^{+}(\mathrm{m} / z)$ : Calcd. for $\mathrm{C}_{11} \mathrm{H}_{18} \mathrm{O}_{7} \mathrm{Na}[\mathrm{M}+\mathrm{Na}]^{+} 285.0945$; found, 285.0957 .

\section{Tecomin (6).}

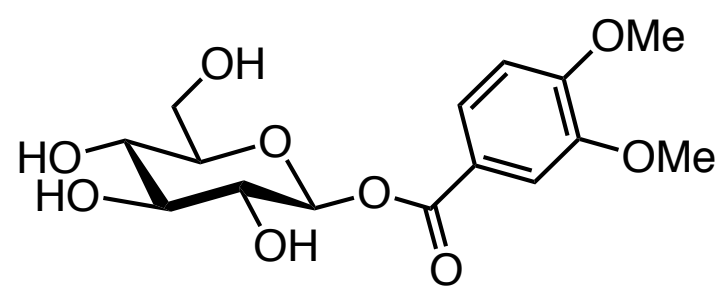

General procedure A for the synthesis of glycoside was followed, starting with $\alpha$-D-glucose $(3.24 \mathrm{~g}, 18$ mmol, 3.0 equiv.), 3,4-dimethoxybenzoic acid (1.09 mg, $6.0 \mathrm{mmol}, 1.0$ equiv.), DIAD (2.36 mL, $12 \mathrm{mmol}$, 2.0 equiv.), and $\mathrm{Ph}_{3} \mathrm{P}$ (3.15 g, $12 \mathrm{mmol}, 2.0$ equiv.) in 1,4-dioxane (600 mL) for $30 \mathrm{~min}$, affording tecomin (6) $(1.44 \mathrm{~g}, 70 \%, \alpha / \beta=1 / 99)$ as a white amorphous powder after purification by flash chromatography, eluting with $\mathrm{CHCl}_{3} / \mathrm{MeOH}(50: 1$ to $5: 1 \mathrm{v} / \mathrm{v})$. Analytical data: $[\boldsymbol{\alpha}]_{\mathbf{D}}{ }^{20}=-15(c 1.0, \mathrm{MeOH}) ;$ TLC $\left(\mathrm{CHCl}_{3} / \mathrm{MeOH} 5: 1 \mathrm{v} / \mathrm{v}\right): \mathrm{R}_{f}=0.39 ;{ }^{1} \mathbf{H}$ NMR $\left(400 \mathrm{MHz}\right.$, acetone- $\left.d_{6}+\mathrm{D}_{2} \mathrm{O}\right) \delta: 7.71(\mathrm{dd}, J=8.7,2.3 \mathrm{~Hz}, 1 \mathrm{H})$, $7.58(\mathrm{~d}, J=1.8 \mathrm{~Hz}, 1 \mathrm{H}), 7.08(\mathrm{~d}, J=8.7 \mathrm{~Hz}, 1 \mathrm{H}), 5.71(\mathrm{~d}, J=7.8 \mathrm{~Hz}, 1 \mathrm{H}), 3.90(\mathrm{~s}, 3 \mathrm{H}), 3.87(\mathrm{~s}, 3 \mathrm{H}), 3.86-$ $3.80(\mathrm{~m}, 1 \mathrm{H}), 3.71-3.66(\mathrm{~m}, 1 \mathrm{H}), 3.55-3.45(\mathrm{~m}, 4 \mathrm{H}) ;{ }^{13} \mathbf{C}$ NMR $\left(100 \mathrm{MHz}\right.$, acetone- $\left.d_{6}+\mathrm{D}_{2} \mathrm{O}\right) \delta: 165.5,154.8$, 149.8, 124.8, 122.6, 113.1, 111.6, 95.8, 78.4, 77.6, 73.6, 70.8, 62.2, 56.2, 56.1; IR $\left(\mathrm{KBr}, \mathrm{cm}^{-1}\right): 3367,1705$, 1600, 1517, 1276, 1223, 1083, 1023, 761; HRMS-ESI ${ }^{+}(\mathrm{m} / \mathrm{z})$ : Calcd. for $\mathrm{C}_{15} \mathrm{H}_{20} \mathrm{O}_{9} \mathrm{Na}[\mathrm{M}+\mathrm{Na}]^{+} 367.1000$; found, 367.1006 . 


\section{Perilloside B (7).}

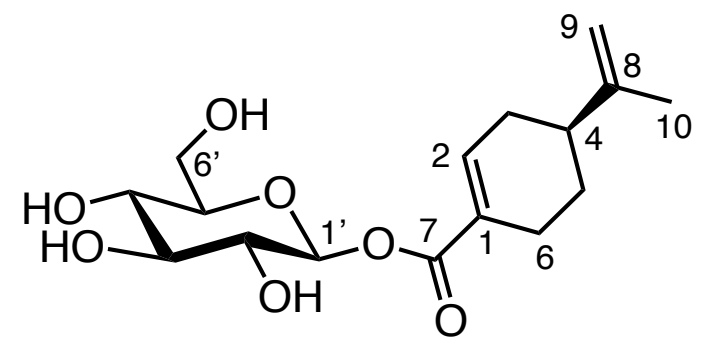

General procedure A for the synthesis of glycoside was followed, starting with $\alpha$-D-glucose (200 mg, 1.1 mmol, 3.0 equiv.), $(S)$-(-)-perillic acid (61.5 mg, 0.37 mmol, 1.0 equiv.), DIAD (145 $\mu \mathrm{L}, 0.74$ mmol, 2.0 equiv.), and $\mathrm{Ph}_{3} \mathrm{P}$ (194 mg, $0.74 \mathrm{mmol}, 2.0$ equiv.) in 1,4-dioxane (37 mL) for $30 \mathrm{~min}$ affording perilloside $\mathrm{B}$ (7) $(74.0 \mathrm{mg}, 61 \%, \alpha / \beta=1 / 99)$ as a white amorphous powder after purification by flash chromatography, eluting with $\mathrm{CHCl}_{3} / \mathrm{MeOH}(50: 1$ to $5: 1 \mathrm{v} / \mathrm{v})$. Analytical data: $[\alpha]_{\mathbf{D}}{ }^{\mathbf{2 0}}=-52(c 0.6, \mathrm{MeOH})$, reported $[\alpha]_{\mathbf{D}}{ }^{22}=$ $-57.1(c \text { 0.645, MeOH})^{6}$; TLC $\left(\mathrm{CHCl}_{3} / \mathrm{MeOH} 5: 1 \mathrm{v} / \mathrm{v}\right): \mathrm{R}_{f}=0.35 ;{ }^{1} \mathbf{H}$ NMR (400 MHz, methanol- $\left.d_{4}\right) \delta$ : 7.15 (br s, 1H), 5.53 (d, $J=7.2 \mathrm{~Hz}, 1 \mathrm{H}), 4.77-4.74(\mathrm{~m}, 2 \mathrm{H}), 3.83$ (dd, $J=10.4,2.0 \mathrm{~Hz}, 1 \mathrm{H}), 3.70-3.66$ (m, $1 \mathrm{H}), 3.44-3.36(\mathrm{~m}, 4 \mathrm{H}), 2.49-2.10(\mathrm{~m}, 5 \mathrm{H}), 1.92-1.88(\mathrm{~m}, 1 \mathrm{H}), 1.76(\mathrm{~s}, 3 \mathrm{H}), 1.54-1.48(\mathrm{~m}, 1 \mathrm{H}) ;{ }^{13} \mathbf{C} \mathbf{N M R}$ (100 MHz, methanol- $\left.d_{4}\right) \delta:$ 167.3, 150.0, 142.3, 130.5, 109.8, 95.7, 78.8, 78.1, 74.0, 71.1, 62.3, 41.4, 32.2, 28.2, 25.4, 20.9; IR $\left(\mathrm{KBr}, \mathrm{cm}^{-1}\right): 3390,2933,1718,1698,1647,1376,1256,1081,890,739,703$; HRMS-FAB ${ }^{+}(\mathrm{m} / \mathrm{z})$ : Calcd. for $\mathrm{C}_{16} \mathrm{H}_{25} \mathrm{O}_{7}[\mathrm{M}+\mathrm{H}]^{+}$329.1600; found, 329.1603 .

\section{Comparison of ${ }^{13} \mathrm{C}$ NMR between isolated ${ }^{6}$ and synthetic perilloside $B(7)$.}

\begin{tabular}{|l|l|}
\hline Synthetic $\mathbf{7}\left(\mathbf{1 0 0} \mathbf{~ M H z}\right.$, methanol- $\left.\boldsymbol{d}_{\mathbf{4}}\right)$ & Isolated sample $^{\mathbf{6}} \mathbf{6 7 . 8} \mathbf{~ M H z}$, methanol- $\left.\boldsymbol{d}_{\mathbf{4}}\right)$ \\
\hline 20.9 & $20.9(\mathrm{C} 10)$ \\
\hline 25.4 & $25.3(\mathrm{C} 6)$ \\
\hline 28.2 & $28.1(\mathrm{C} 5)$ \\
\hline 32.2 & $32.2(\mathrm{C} 3)$ \\
\hline 41.4 & $41.2(\mathrm{C} 4)$ \\
\hline 62.3 & $62.2\left(\mathrm{C} 6^{\prime}\right)$ \\
\hline 71.1 & $70.9\left(\mathrm{C} 4^{\prime}\right)$ \\
\hline 74.0 & $73.9\left(\mathrm{C} 2^{\prime}\right)$ \\
\hline 78.1 & $77.9\left(\mathrm{C} 5^{\prime}\right)$ \\
\hline 78.8 & $78.6\left(\mathrm{C} 3^{\prime}\right)$ \\
\hline 95.7 & $95.7\left(\mathrm{C} 1^{\prime}\right)$ \\
\hline 109.8 & $109.8(\mathrm{C} 9)$ \\
\hline 130.5 & $130.4(\mathrm{C} 1)$ \\
\hline 142.3 & $142.3(\mathrm{C} 2)$ \\
\hline 150.0 (correct) & $\mathbf{1 5 9 . 9}(\mathbf{C} 8)$ (error) \\
\hline 167.3 & $167.2(\mathrm{C} 7)$ \\
\hline
\end{tabular}

The ${ }^{13} \mathrm{C}$ chemical shift that differs significantly from that of the reported natural product is shown in bold. This difference comes from a human careless mistake in the assignment of isolated natural product. 
1-O-(3,4,5-Tribenzyloxybenzoyl)- $\beta$-D-glucopyranoside (S1).

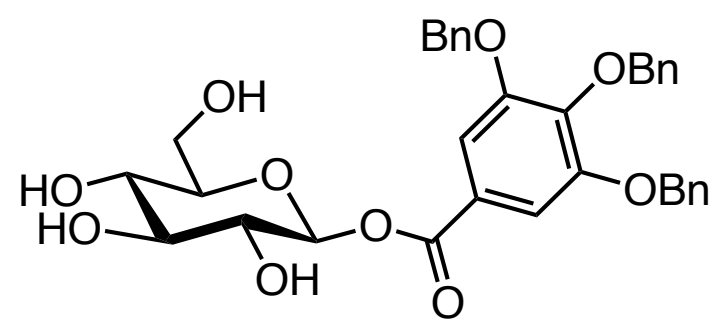

General procedure A for the synthesis of glycoside was followed, starting with $\alpha$-D-glucose (300 mg, 1.7 mmol, 3.0 equiv.), 3,4,5-tris(benzyloxy)benzoic acid ${ }^{1}$ ( $244 \mathrm{mg}, 0.565 \mathrm{mmol}, 1.0$ equiv.), DIAD (219 $\mu \mathrm{L}, 1.1$ mmol, 2.0 equiv.), and $\mathrm{Ph}_{3} \mathrm{P}$ (291 mg, $1.1 \mathrm{mmol}, 2.0$ equiv.) in 1,4-dioxane (55 mL) for $1 \mathrm{~h}$, affording corresponding the glucoside $(237 \mathrm{mg}, 71 \%, \alpha / \beta=1 / 99)$ as a white amorphous powder after purification by flash chromatography, eluting with $\mathrm{CHCl}_{3} / \mathrm{MeOH}(50: 1$ to $5: 1 \mathrm{v} / \mathrm{v})$. Analytical data: $[\alpha]_{\mathbf{D}}{ }^{21}=-4.1(c 1.0$, acetone); TLC $\left(\mathrm{CHCl}_{3} / \mathrm{MeOH} 5: 1 \mathrm{v} / \mathrm{v}\right): \mathrm{R}_{f}=0.43 ;{ }^{1} \mathbf{H}$ NMR $\left(400 \mathrm{MHz}\right.$, acetone- $\left.d_{6}+\mathrm{D}_{2} \mathrm{O}\right) \delta: 7.55-7.26(\mathrm{~m}$, $17 \mathrm{H}), 5.70(\mathrm{~d}, J=7.6 \mathrm{~Hz}, 1 \mathrm{H}), 5.23(\mathrm{~s}, 4 \mathrm{H}), 5.14(\mathrm{~s}, 2 \mathrm{H}), 3.84-3.81(\mathrm{~m}, 1 \mathrm{H}), 3.71-3.67(\mathrm{~m}, 1 \mathrm{H}), 3.55-3.53$ (m, 2H), 3.48-3.46 (m, 2H); ${ }^{13} \mathbf{C}$ NMR (150 MHz, acetone- $\left.d_{6}\right) \delta: 165.1,153.6,143.6,138.7,138.0,129.4$, 129.2 , 129.0, 128.8, 128.69, 128.65, 125.7, 110.1, 96.1, 78.6, 78.0, 75.5, 73.9, 71.8, 71.2, 62.5; IR $(\mathrm{KBr}$, $\mathrm{cm}^{-1}$ ): 3331, 3203, 2367, 1714, 1647, 1591, 1503, 1427, 1335, 1252, 1217, 1133, 1085, 985, 858, 730, 690; HRMS-ESI ${ }^{-}(\mathrm{m} / \mathrm{z})$ : Calcd. for $\mathrm{C}_{34} \mathrm{H}_{34} \mathrm{O}_{10} \mathrm{Cl}[\mathrm{M}+\mathrm{Cl}]^{-}$637.1840; found, 637.1835.

\section{$\beta$-Glucogallin (8).}

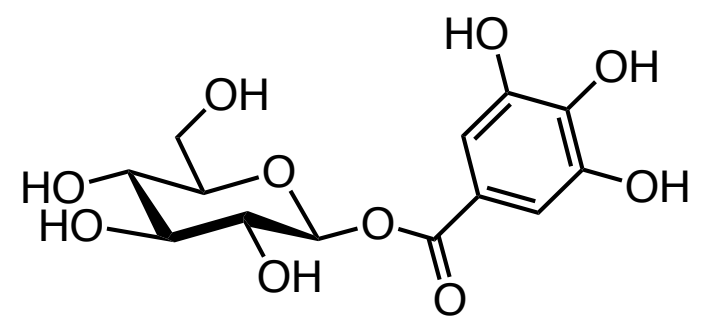

A $10 \mathrm{~mL}$ round-bottom flask was charged with $\mathbf{S 1}(200 \mathrm{mg}, 0.33 \mathrm{mmol}, 1.0$ equiv. $)$ and $\mathrm{Pd}(\mathrm{OH})_{2} / \mathrm{C}(10$ wt.\%, $20.0 \mathrm{mg}$ ). $3.3 \mathrm{~mL}$ of EtOH/acetone (4/1) was added and the atmosphere was replaced by $\mathrm{H}_{2}$ (balloon). The reaction mixture was stirred at $\mathrm{rt}$ for $4 \mathrm{~h}$ until TLC analysis indicated complete consumption of the starting material. The resulting suspension was filtered and washed with acetone. The filtrate was concentrated in vacuo to give $\beta$-glucogallin $(\mathbf{8})(105 \mathrm{mg}, 95 \%)$ as a pale gray amorphous powder. Analytical data: $[\boldsymbol{\alpha}]_{\mathbf{D}}{ }^{\mathbf{2 1}}=-9.8(c 1.0, \mathrm{MeOH})$, reported $[\boldsymbol{\alpha}]_{\mathbf{D}}{ }^{\mathbf{2 0}}=-8.0(c \text { } 0.1, \mathrm{MeOH})^{7} ;{ }^{1} \mathbf{H}$ NMR $\left(600 \mathrm{MHz}\right.$, methanol- $\left.d_{4}\right)$ $\delta: 7.12(\mathrm{~s}, 2 \mathrm{H}), 5.65(\mathrm{~d}, J=7.8 \mathrm{~Hz}, 1 \mathrm{H}), 3.85(\mathrm{dd}, J=12.0 \mathrm{~Hz}, 2.4 \mathrm{~Hz}, 1 \mathrm{H}), 3.70$ (dd, $J=12.6,4.8 \mathrm{~Hz}, 1 \mathrm{H})$, 3.48-3.47 (m, 2H), 3.43-3.40 (m, 2H); ${ }^{13}$ C NMR (150 MHz, methanol- $\left.d_{4}\right) \delta: 167.1,146.5,140.3,120.8$, 110.5, 96.0, 78.8, 78.2, 74.1, 71.1, 62.3; IR (KBr, $\left.\mathrm{cm}^{-1}\right)$ : 3550, 3307, 2925, 1714, 1594, 1451, 1395, 1316, 1281, 1077, 1021, 711; HRMS-ESI $^{-}(\mathrm{m} / \mathrm{z})$ : Calcd. for $\mathrm{C}_{13} \mathrm{H}_{15} \mathrm{O}_{10}[\mathrm{M}-\mathrm{H}]^{-} 331.0658$; found, 331.0660. 
Skimmin (9).

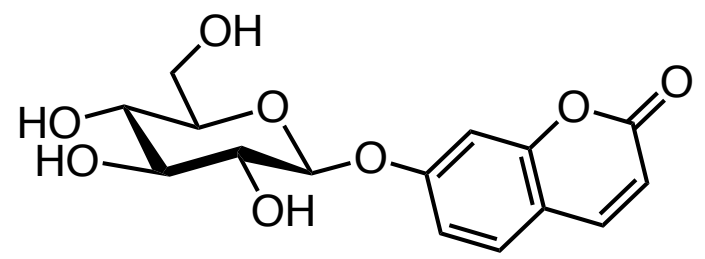

A round-bottom flask was charged with $\alpha$-D-glucose $(101 \mathrm{mg}, 0.56 \mathrm{mmol}, 3.0$ equiv.) and dehydrated 1,4-dioxane $(19 \mathrm{~mL})$ under an Ar atmosphere. After ultrasound irradiation of the suspension of $\alpha$-D-glucose in 1,4-dioxane for $15 \mathrm{~min}$, umbelliferone (30.5 mg, $0.19 \mathrm{mmol}, 1.0$ equiv.) and $\mathrm{Ph}_{3} \mathrm{P}$ (98.6 mg, $0.38 \mathrm{mmol}$, 2.0 equiv.) were added. DIAD ( $74 \mu \mathrm{L}, 0.38 \mathrm{mmol}, 2.0$ equiv.) was added dropwise by syringe and the reaction mixture was stirred at $55^{\circ} \mathrm{C}$, using oil bath. After stirring vigorously for $15 \mathrm{~min}$, DIAD $(74 \mu \mathrm{L}, 0.38$ mmol, 2.0 equiv.) and $\mathrm{Ph}_{3} \mathrm{P}$ (98.6 mg, $0.38 \mathrm{mmol}, 2.0$ equiv.) were added again in the same manner and stirred at $55^{\circ} \mathrm{C}$ for $45 \mathrm{~min}$. The reaction mixture was quenched with $3 \mathrm{~mL}$ of $\mathrm{MeOH}$, stirred for $5 \mathrm{~min}$, and concentrated in vacuo at $40{ }^{\circ} \mathrm{C}$ (water bath) to give a residue. The residue was directly purified by flash column chromatography $\left(\mathrm{SiO}_{2}, \mathrm{CHCl}_{3} / \mathrm{MeOH} 10: 1\right.$ to $\left.5: 1 \mathrm{v} / \mathrm{v}\right)$ to give the skimmin (9) $(37.2 \mathrm{mg}, 61 \%$, $\alpha / \beta=4: 96)$ as a white amorphous powder. Analytical data: $[\alpha]_{\mathbf{D}}{ }^{19}=-177$ (c $\left.0.1, \mathrm{MeOH}\right)$; TLC $\left(\mathrm{CHCl}_{3} / \mathrm{MeOH} 5 / 1 \mathrm{v} / \mathrm{v}\right): \mathrm{R}_{f}=0.29 ;{ }^{1} \mathbf{H}$ NMR $\left(400 \mathrm{MHz}, \mathrm{DMSO}-d_{6}\right) \delta: 8.01(\mathrm{~d}, J=9.6 \mathrm{~Hz}, 1 \mathrm{H}), 7.65(\mathrm{~d}, J=$ $8.7 \mathrm{~Hz}, 1 \mathrm{H}), 7.05(\mathrm{~d}, J=2.3 \mathrm{~Hz}, 1 \mathrm{H}), 7.01$ (dd, $J=11.0,2.3 \mathrm{~Hz}, 1 \mathrm{H}), 6.33$ (d, $J=9.6 \mathrm{~Hz}, 1 \mathrm{H}), 5.43$ (brs, $1 \mathrm{H}$ ), $5.19(\mathrm{~d}, J=4.6 \mathrm{~Hz}, 1 \mathrm{H}), 5.11(\mathrm{~d}, J=5.5 \mathrm{~Hz}, 1 \mathrm{H}), 5.03(\mathrm{~d}, J=7.3 \mathrm{~Hz}, 1 \mathrm{H}), 4.61(\mathrm{t}, J=6.0 \mathrm{~Hz}, 1 \mathrm{H}), 3.70(\mathrm{dd}$, $J=10.1,5.0 \mathrm{~Hz}, 1 \mathrm{H}), 3.47-3.42(\mathrm{~m}, 2 \mathrm{H}), 3.28-3.13(\mathrm{~m}, 3 \mathrm{H}) ;{ }^{13} \mathbf{C}$ NMR (100 MHz, DMSO- $\left.d_{6}\right) \delta: 160.3$, $160.2,155.1,144.3,129.5,113.7,113.3,113.2,103.2,100.0,77.2,76.5,73.1,69.6,60.6$; IR $(\mathrm{KBr}$, $\left.\mathrm{cm}^{-1}\right): 3356,2915,1717,1621,1404,1281,1073,1016,838$; HRMS-ESI ${ }^{+}(\mathrm{m} / \mathrm{z})$ : Calcd. for $\mathrm{C}_{15} \mathrm{H}_{17} \mathrm{O}_{8}$ $[\mathrm{M}+\mathrm{H}]^{+} 325.0918$; found, 325.0895 . 


\section{KIE MEASUREMENTS (Table S3)}

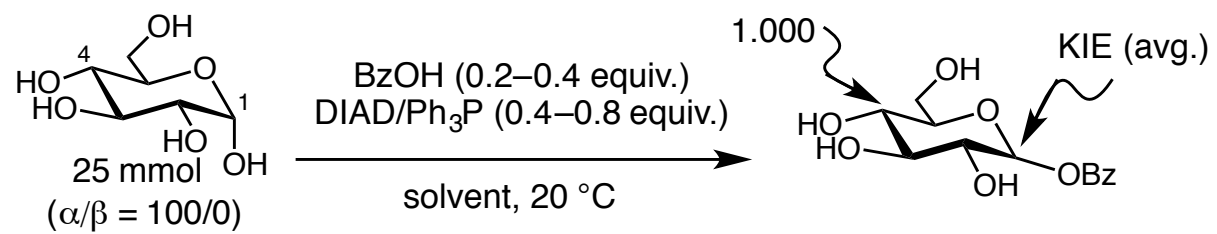

\begin{tabular}{ccccccc}
\hline Entry & Solvent & $\mathrm{BzOH}$ (eq.) & $\mathrm{DIAD} \mathrm{Ph}_{3} \mathrm{P}$ (eq.) & Conversion $(\%)^{a}$ & $\alpha / \beta$ & $\mathrm{KIE}\left(20^{\circ} \mathrm{C}\right)$ \\
\hline 1 & 1,4-dioxane & 0.2 & 0.4 & 14.6 & $1 / 99$ & 1.026 \\
2 & 1,4-dioxane & 0.2 & 0.4 & 16.6 & $1 / 99$ & 1.033 \\
3 & 1,4-dioxane & 0.4 & 0.8 & 25.7 & $1 / 99$ & 1.026 \\
average & & & & & & 1.028 \\
\hdashline 4 & DMF & 0.2 & 0.4 & 8.9 & $60 / 40$ & 1.005 \\
5 & DMF & 0.2 & 0.4 & 6.2 & $63 / 37$ & 1.000 \\
6 & DMF & 0.4 & 0.8 & 9.4 & $58 / 42$ & 0.998 \\
average & & & & & & 1.001 \\
\hline
\end{tabular}

aDetermined by crude ${ }^{1} \mathrm{H}$ NMR using 1,3,5-trimethoxybenzene as an internal standard.

Primary ${ }^{13} \mathrm{C}$ KIE was determined for the formation of glycoside using natural abundance NMR method. ${ }^{8} \mathrm{~A}$ round-bottom flask was charged with powdered $\alpha$-D-glucose and dehydrated 1,4-dioxane $(0.03 \mathrm{M}$ of $\alpha$-D-glucose) under an Ar atmosphere. After ultrasound irradiation of the suspension of $\alpha$-D-glucose in 1,4-dioxane for $15 \mathrm{~min}$, benzoic acid and $\mathrm{Ph}_{3} \mathrm{P}$ were added. DIAD was added dropwise by syringe and the resulting mixture was stirred vigorously at $\mathrm{rt}$ for $30 \mathrm{~min}$. The reaction mixture was quenched with $\mathrm{MeOH}$, stirred for $5 \mathrm{~min}$, and concentrated in vacuo at $40{ }^{\circ} \mathrm{C}$ (water bath) to give a residue. The residue was directly purified by flash column chromatography $\left(\mathrm{SiO}_{2}, \mathrm{CHCl}_{3} / \mathrm{MeOH} 50: 1\right.$ to 5:1 v/v) to give the glycoside.

$$
\mathrm{KIE}=\frac{\ln (1-F)}{\ln \left[1-\left(F R_{p} / R_{0}\right)\right]}
$$

Primary ${ }^{13} \mathrm{C}$ KIE of product was calculated from equation (1). ${ }^{9} F$ is the conversion of $\alpha$-D-glucose into the glycoside determined by crude ${ }^{1} \mathrm{H}$ NMR using 1,3,5-trimethoxybenzene as an internal standard. $R_{p}$ is the molar activity of the minor isotope $\left({ }^{13} \mathrm{C}\right)$ in the product, which was determined by integration of anomeric carbon $(\mathrm{C} 1)$ of the glycoside against the $\mathrm{C} 4$ carbon of it. $R_{0}$ is the molar activity of the minor isotope $\left({ }^{13} \mathrm{C}\right)$ in the starting material ( $\alpha$-D-glucose), determined by integration of the sum of anomeric carbon $(C 1)$ in $\alpha$ - and $\beta$-D-glucose against the $\mathrm{C} 4$ carbon of them, because a small amount of $\alpha$-D-glucose was epimerized to $\beta$-D-glucose during the acquisition of ${ }^{13} \mathrm{C}$ NMR spectra in DMSO- $d_{6}$ at $300 \mathrm{~K}$ ( $4 \mathrm{~h} 40 \mathrm{~min}$ ). Assuming that $\mathrm{KIE}$ at $\mathrm{C} 4$ of glucopyranoside ring is relatively small in glycosylation, ${ }^{10}$ we chose the $\mathrm{C} 4$ carbon as an internal standard of ${ }^{13} \mathrm{C}$ concentration. The determined $\alpha$-primary ${ }^{13} \mathrm{C} \mathrm{KIE}$ was the average of three runs. 


\section{NMR EXPERIMENTS (Figure S3)}

Carbon NMR spectra were recorded on a Bruker Avance $800\left(200 \mathrm{MHz}\right.$ operating frequency for ${ }^{13} \mathrm{C}$ measurement), equipped with a $5 \mathrm{~mm}$-TCI $800 \mathrm{MHz}$ cryogenic probe. Shigemi $5 \mathrm{~mm}$ NMR tubes (PS-003) were used. Samples of $\alpha$-D-glucose (200 mg) and 1-O-benzoyl-D-glucopyranoside (200-250 mg) were dissolved in DMSO- $d_{6}$ (D 99.9\%, Cambridge isotope Laboratories, Inc.). The temperature was set to $300 \mathrm{~K}$. For the acquisition of the quantitative ${ }^{13} \mathrm{C}$-NMR spectra, direct carbon detection was obtained using $90^{\circ}$ pulse with their carrier frequency adjusted at the middle of the peaks of sugar carbons $(78.05 \mathrm{ppm})$ to equilibrate offset effects. Inverse gated bi-level decoupling via Waltz-64 sequence was applied with carrier frequency between the neighboring protons, to avoid creation of heteronuclear Overhauser effect and to eliminate decoupling sidebands. Long enough acquisitions (2.93 s) and relaxation delays (30 s) were employed. The signal-to-noise ratios were between 1863 and 2658.

\section{Quantitative ${ }^{13} \mathrm{C}$ NMR spectrum of $\alpha$-D-glucose, SINO $=2658$.}

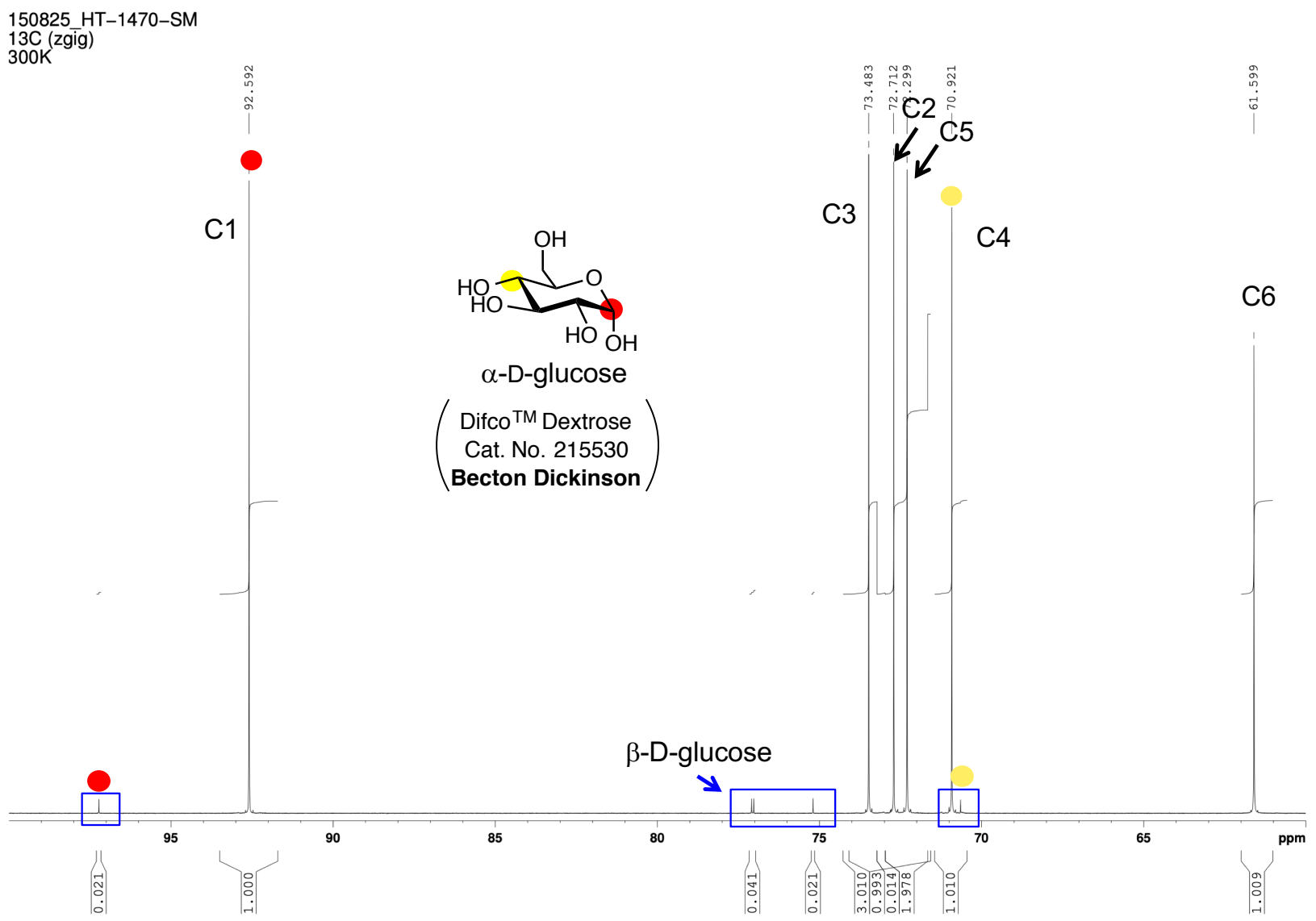


Quantitative ${ }^{13} \mathrm{C}$ NMR spectrum of the glycoside (Table S3, entry $1,14.6 \%$ conv.), SINO = 1863 .
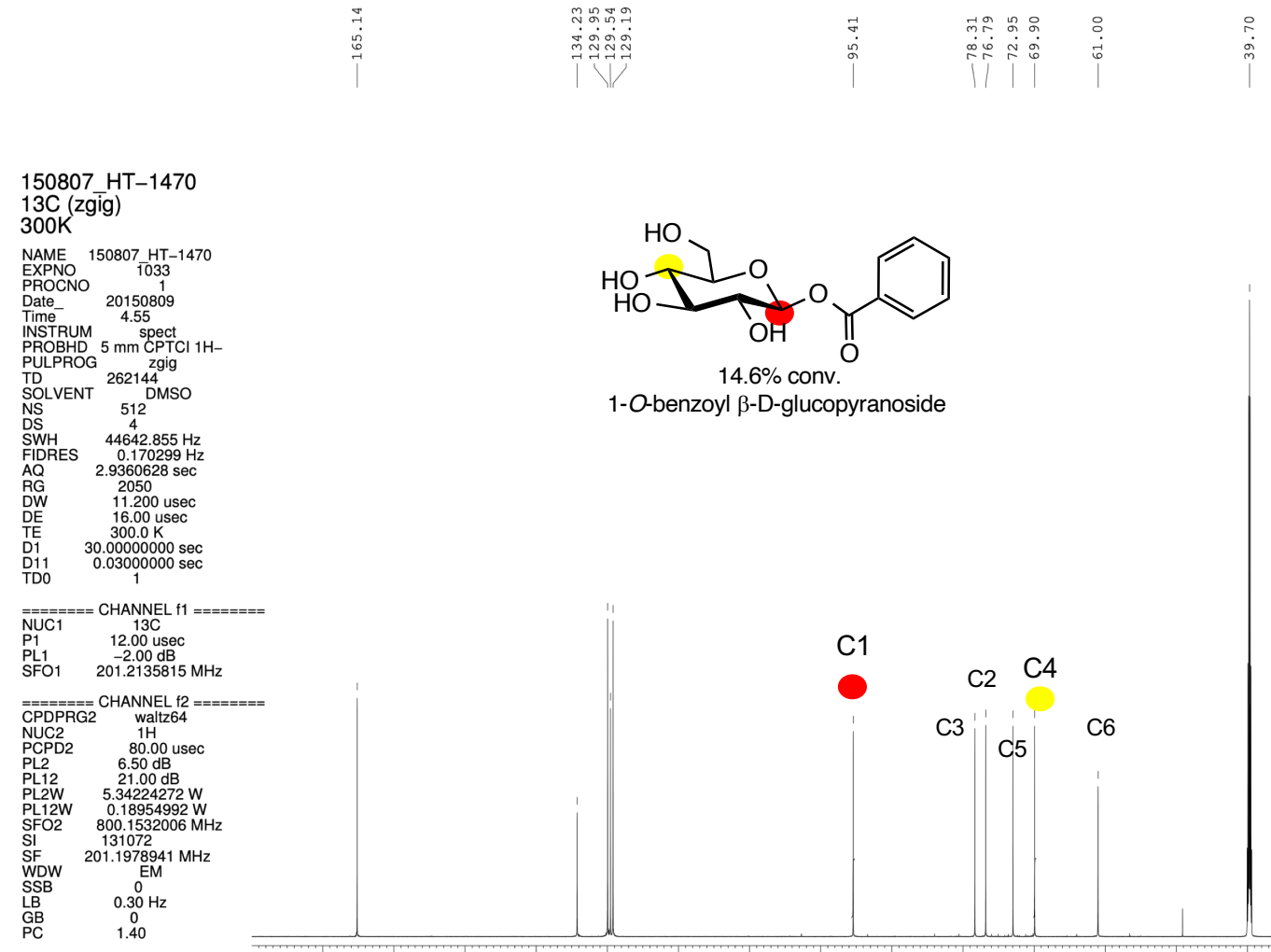

i

Quantitative ${ }^{13} \mathrm{C}$ NMR spectrum of the glycoside (Table S3, entry 3, 25.7\% conv.), SINO = 2418 .

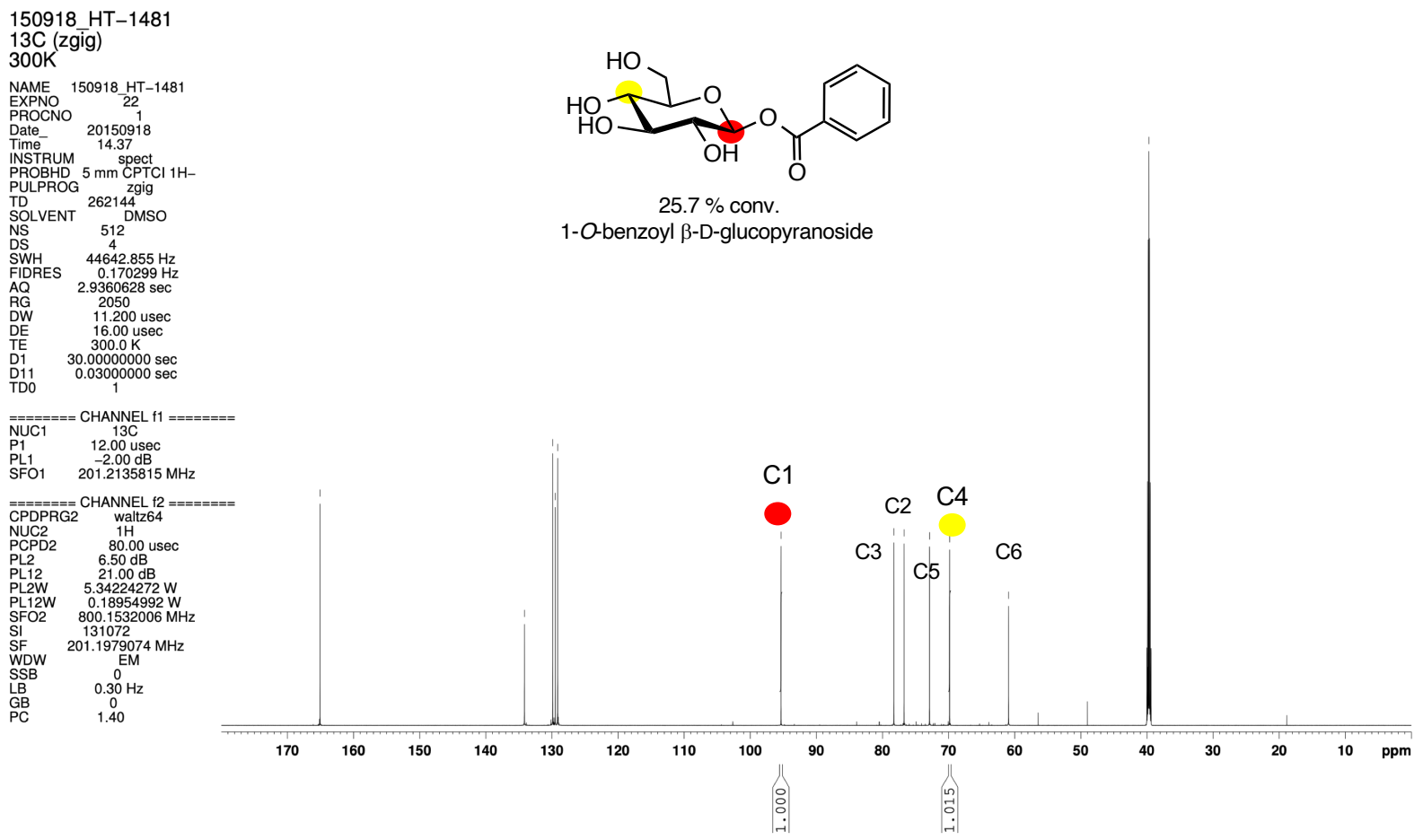


Quantitative ${ }^{13} \mathrm{C}$ NMR spectrum of the glycoside (Table S3, entry $2,16.6 \%$ conv.), SINO = 1913.
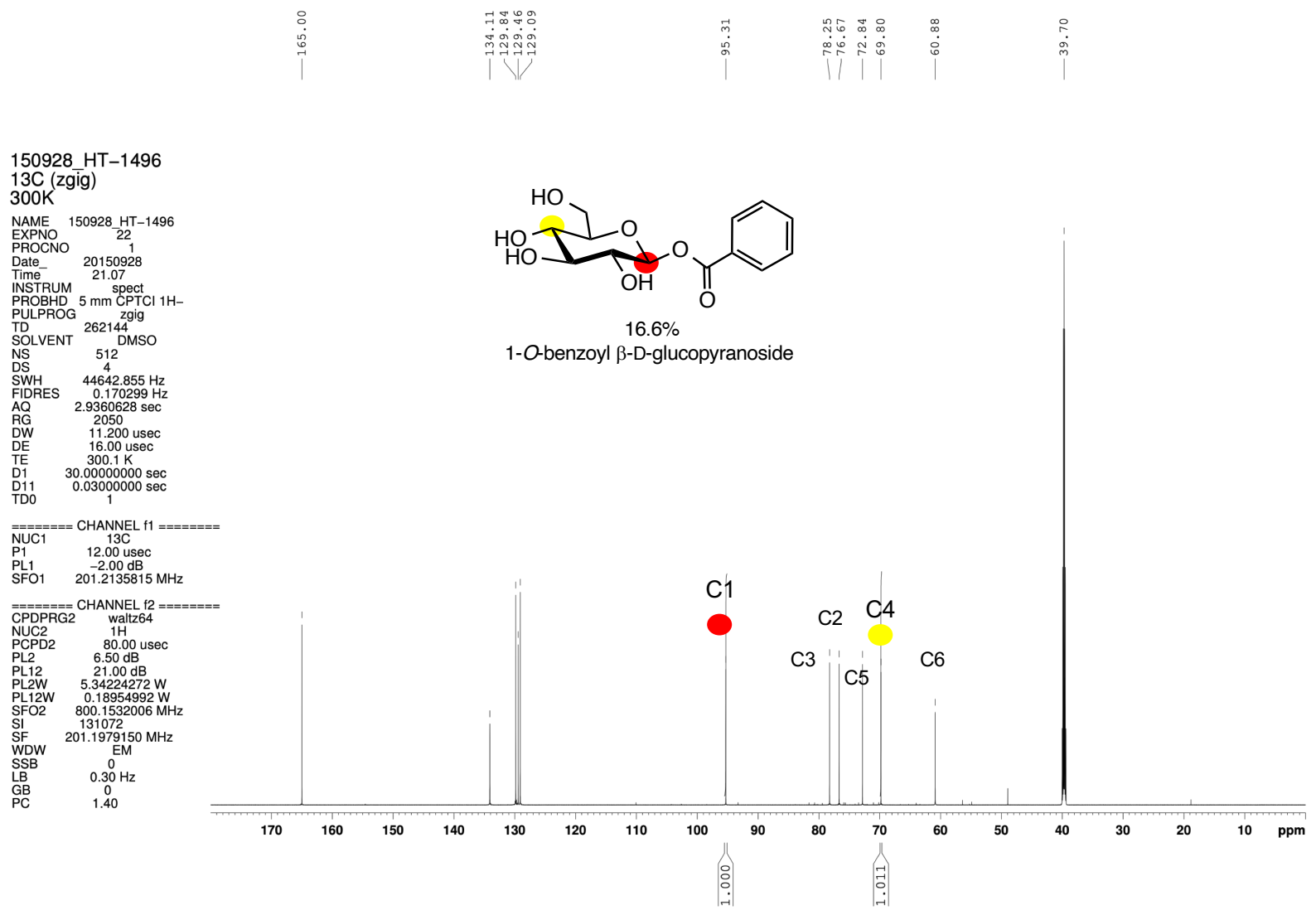

Quantitative ${ }^{13} \mathrm{C}$ NMR spectrum of the glycosides (Table S3, entry 4, 8.9\% conv.), SINO=2397.
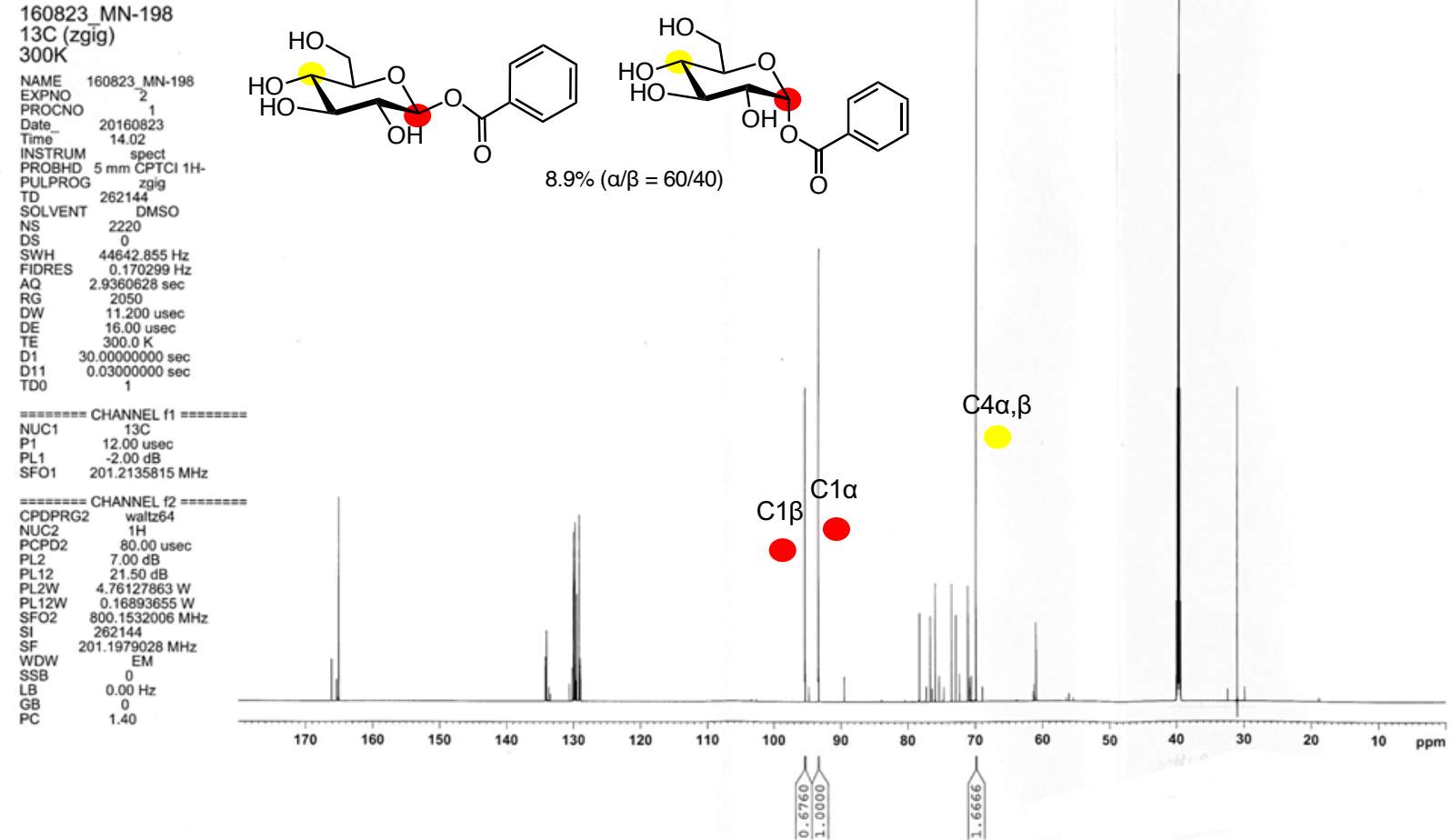
Quantitative ${ }^{13} \mathrm{C}$ NMR spectrum of the glycosides (Table S3, entry 6, 9.4\% conv.), SINO = 2439 .

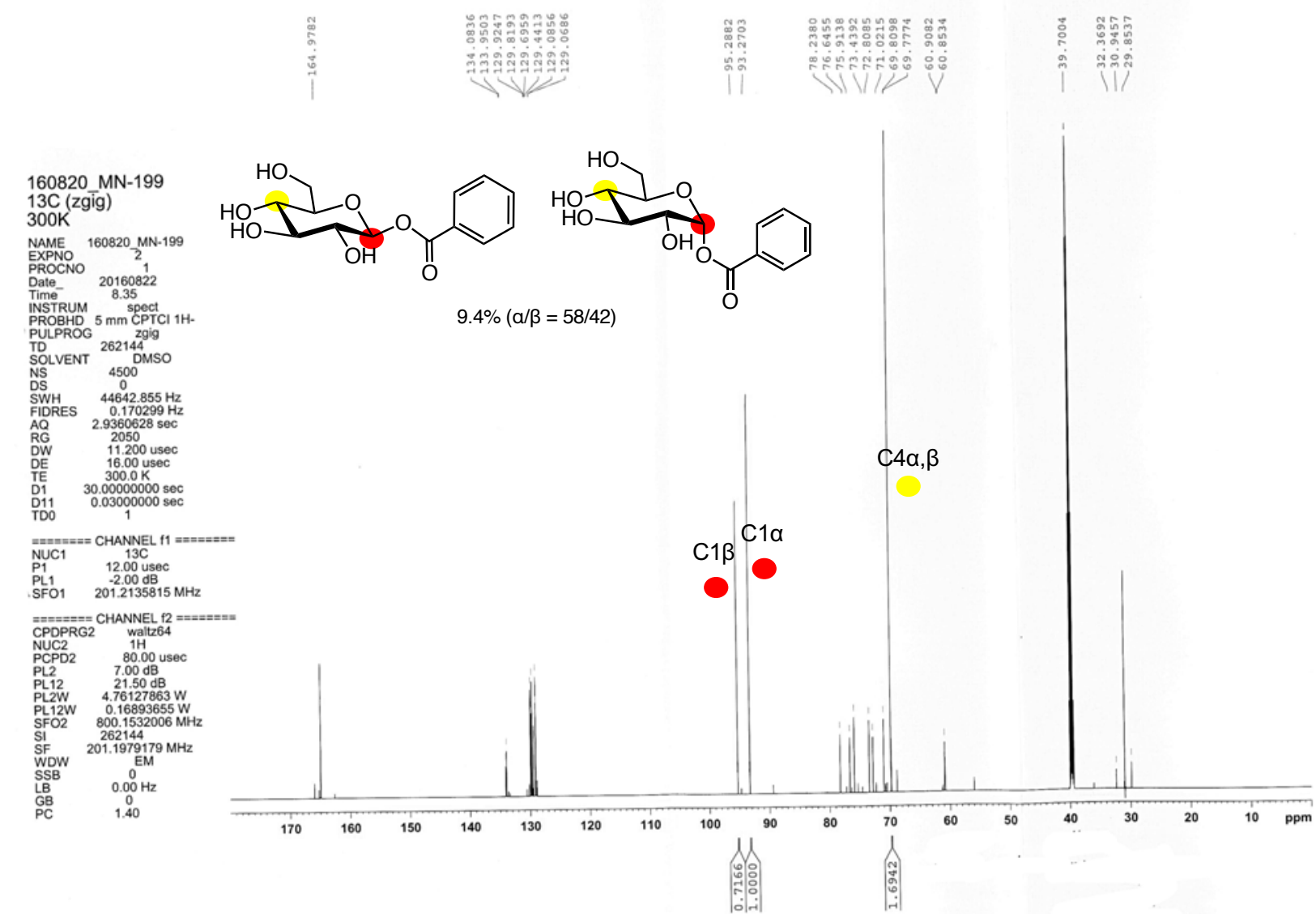

Quantitative ${ }^{13} \mathrm{C}$ NMR spectrum of the glycosides (Table S3, entry 5, 6.2\% conv.), SINO = 1925.
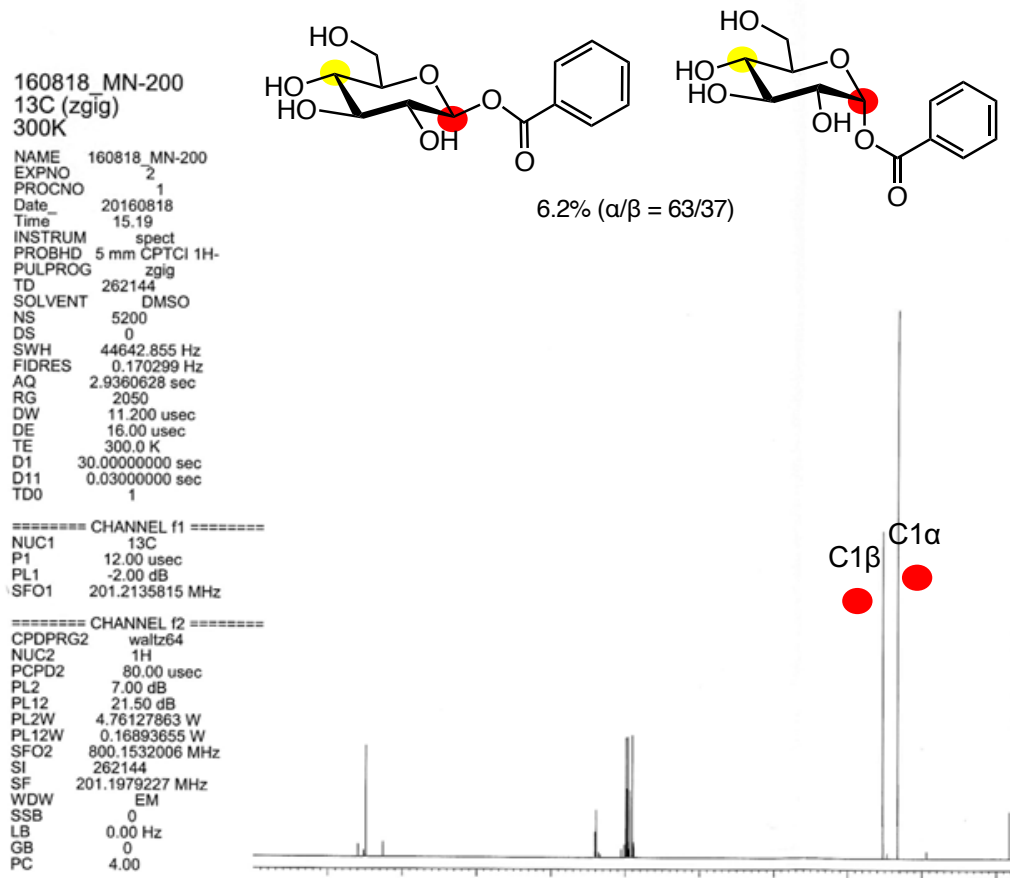

$6.2 \%(\alpha / \beta=63 / 37)$

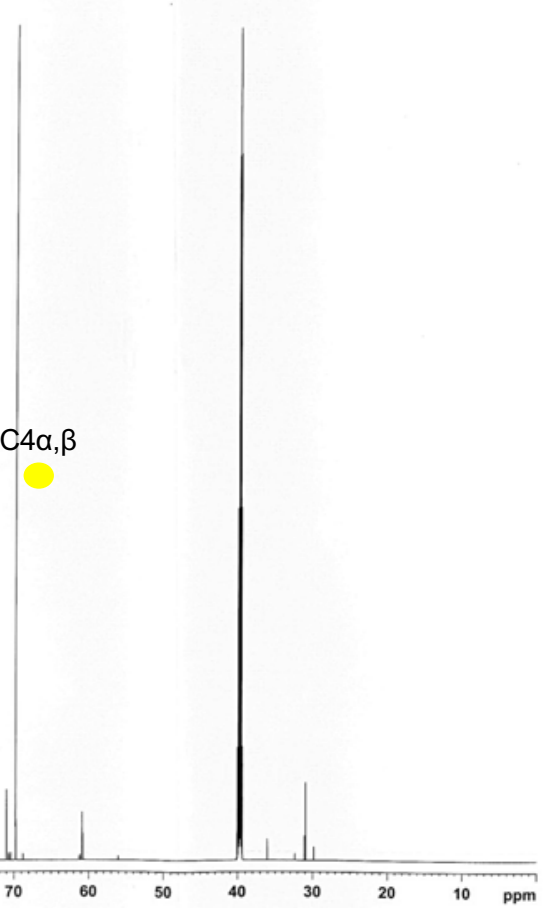




\section{X-RAY CRYSTAL STRUCTURAL ANALYSIS OF $2 f$}

\section{X-ray crystallography}

Single crystal of $\mathbf{2 f}$ was obtained by cooling its saturated hot solution in $\mathrm{CH}_{3} \mathrm{CN}$ to $15{ }^{\circ} \mathrm{C}$. The crystal data of $2 \mathbf{f}$ was collected on a Rigaku Saturn 70 CCD diffractometer with a VariMax Mo Optic System using a Mo $\mathbf{K} \boldsymbol{\alpha}$ radiation $(\lambda=0.71070 \AA)$. The reflection data for $\mathbf{2 f}$ was integrated, scaled, and averaged by using the HKL-2000. ${ }^{11}$ Semi-empirical absorption correction was applied using the program of MULABS. ${ }^{12}$ The structure was solved by a direct method (SIR2004) ${ }^{13}$ and refined by full-matrix least square method of $F^{2}$ for all reflections with the Shelx program package (SHELXL-97). ${ }^{14}$ All hydrogen atoms were refined isotropically, while all other atoms were refined anisotropically. CCDC-1445049 (2f) contains the supplementary crystallographic data for this paper. This data can be obtained free of charge from The Cambridge Crystallographic Data Center via www.ccdc.cam.ac.uk/data_request/cif.

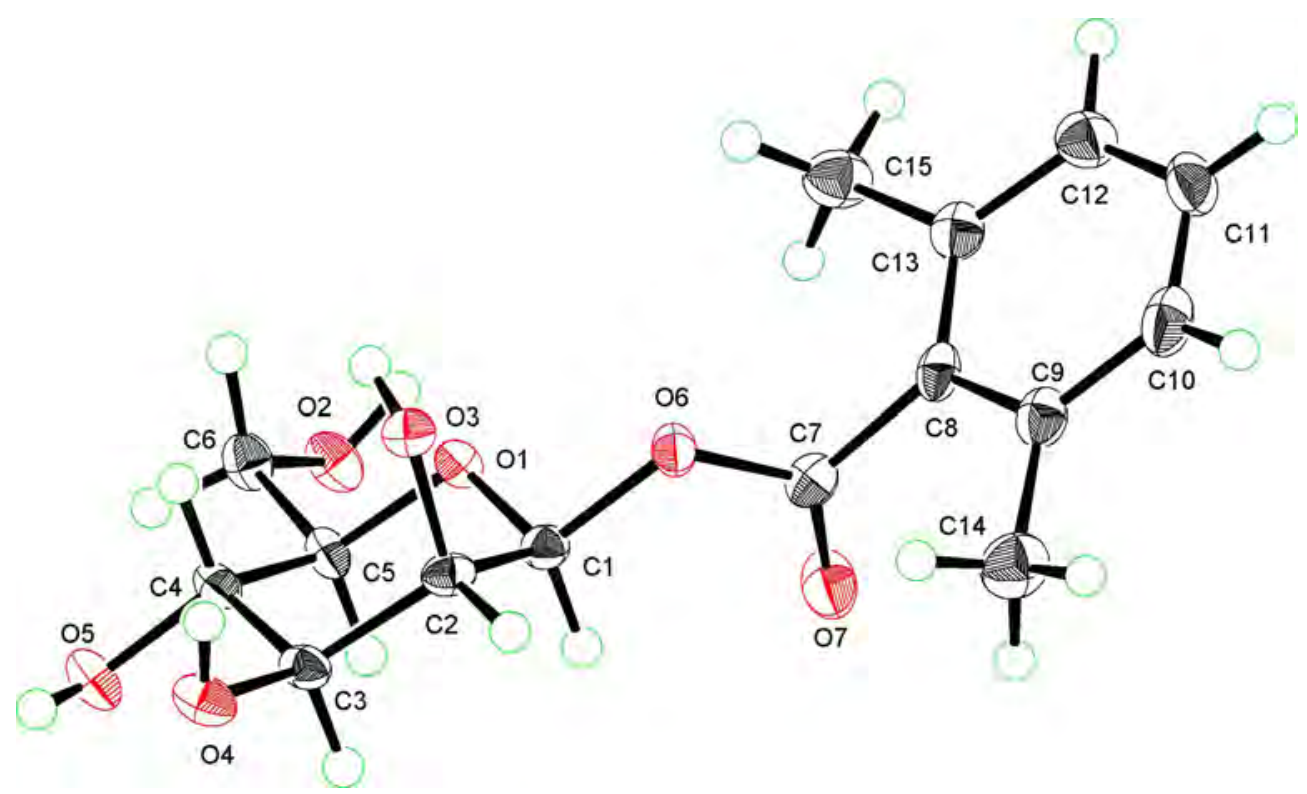

Crystal structure of $\mathbf{2} \mathbf{f}$ ( $50 \%$ probability). 
Table 1. Crystal data and structure refinement for HT1557 (Compound 2f)

CCDC No.

Identification code

Empirical formula

Formula weight

Temperature

Wavelength

Crystal system

Space group

Unit cell dimensions

Volume

Z

Density (calculated)

Absorption coefficient

$\mathrm{F}(000)$

Crystal size

Theta range for data collection

Index ranges

Reflections collected

Independent reflections

Completeness to theta $=27.50^{\circ}$

Max. and min. transmission

Refinement method

Data / restraints / parameters

Goodness-of-fit on $\mathrm{F}^{2}$

Final $\mathrm{R}$ indices [I $>2 \operatorname{sigma}(\mathrm{I})]$

$\mathrm{R}$ indices (all data)

Absolute structure parameter

Largest diff. peak and hole
1445049

ht1557

C15 H20 O7

312.31

103(2) K

$0.71075 \AA$

Orthorhombic

$P 2{ }_{1} 2_{1} 2_{1}$ (\#19)

$\mathrm{a}=7.9924(2) \AA$

$\alpha=90^{\circ}$.

$\mathrm{b}=8.9411(3) \AA$

$\beta=90^{\circ}$.

$\mathrm{c}=20.9681(7) \AA$

$\gamma=90^{\circ}$.

1498.40(8) $\AA^{3}$

4

$1.384 \mathrm{Mg} / \mathrm{m}^{3}$

$0.110 \mathrm{~mm}^{-1}$

664

$0.30 \times 0.05 \times 0.04 \mathrm{~mm}^{3}$

1.94 to $27.50^{\circ}$.

$-10<=\mathrm{h}<=10,-11<=\mathrm{k}<=11,-27<=\mathrm{l}<=27$

20303

$3454[\mathrm{R}(\mathrm{int})=0.0874]$

$99.9 \%$

0.9956 and 0.9677

Full-matrix least-squares on $\mathrm{F}^{2}$

3454 / 0 / 279

1.021

$\mathrm{R} 1=0.0391, \mathrm{wR} 2=0.0644$

$\mathrm{R} 1=0.0637, \mathrm{wR} 2=0.0710$

$0.7(9)$

0.174 and -0.178 e. $\AA^{-3}$ 
Table 2. Atomic coordinates ( $\left.\times 10^{4}\right)$ and equivalent isotropic displacement parameters $\left(\AA^{2} \times 10^{3}\right)$ for HT1557. $\mathrm{U}(\mathrm{eq})$ is defined as one third of the trace of the orthogonalized $\mathrm{U}^{\mathrm{ij}}$ tensor.

\begin{tabular}{|c|c|c|c|c|}
\hline & $\mathrm{x}$ & $\mathrm{y}$ & $\mathrm{z}$ & $\mathrm{U}(\mathrm{eq})$ \\
\hline $\mathrm{O}(1)$ & $796(1)$ & $2646(1)$ & $6677(1)$ & $18(1)$ \\
\hline $\mathrm{C}(1)$ & 1933(2) & $2851(2)$ & $6168(1)$ & $18(1)$ \\
\hline$C(2)$ & $3628(2)$ & $3333(2)$ & $6411(1)$ & $18(1)$ \\
\hline$C(3)$ & $4273(2)$ & $2122(2)$ & $6855(1)$ & $18(1)$ \\
\hline$C(4)$ & $3017(2)$ & $1807(2)$ & $7375(1)$ & $18(1)$ \\
\hline$C(5)$ & $1310(2)$ & $1438(2)$ & $7081(1)$ & $18(1)$ \\
\hline$C(6)$ & $-4(2)$ & $1228(2)$ & $7586(1)$ & $21(1)$ \\
\hline $\mathrm{O}(2)$ & $-1481(2)$ & $546(2)$ & $7341(1)$ & $24(1)$ \\
\hline $\mathrm{O}(3)$ & $3580(1)$ & $4754(1)$ & $6713(1)$ & $19(1)$ \\
\hline $\mathrm{O}(4)$ & $5879(1)$ & $2462(2)$ & $7115(1)$ & $23(1)$ \\
\hline $\mathrm{O}(5)$ & $3493(2)$ & $550(2)$ & $7750(1)$ & $24(1)$ \\
\hline $\mathrm{O}(6)$ & $1260(1)$ & $4032(1)$ & $5791(1)$ & $20(1)$ \\
\hline$C(7)$ & $382(2)$ & $3637(2)$ & $5267(1)$ & $21(1)$ \\
\hline $\mathrm{O}(7)$ & $153(2)$ & $2357(2)$ & $5112(1)$ & $34(1)$ \\
\hline$C(8)$ & $-268(2)$ & $4942(2)$ & $4906(1)$ & $20(1)$ \\
\hline $\mathrm{C}(9)$ & $618(2)$ & $5483(2)$ & $4383(1)$ & $24(1)$ \\
\hline $\mathrm{C}(10)$ & $-104(3)$ & $6597(2)$ & $4020(1)$ & $30(1)$ \\
\hline $\mathrm{C}(11)$ & $-1669(3)$ & $7168(2)$ & $4166(1)$ & $30(1)$ \\
\hline$C(12)$ & $-2515(2)$ & $6645(2)$ & $4694(1)$ & $28(1)$ \\
\hline $\mathrm{C}(13)$ & $-1825(2)$ & $5540(2)$ & $5077(1)$ & $23(1)$ \\
\hline$C(14)$ & $2340(3)$ & $4884(3)$ & $4228(1)$ & $34(1)$ \\
\hline$C(15)$ & $-2702(3)$ & $5021(3)$ & $5672(1)$ & $32(1)$ \\
\hline
\end{tabular}


Table 3. Bond lengths $[\AA]$ and angles $\left[{ }^{\circ}\right]$ for HT1557.

\begin{tabular}{|c|c|c|c|}
\hline $\mathrm{O}(1)-\mathrm{C}(1)$ & $1.413(2)$ & $\mathrm{C}(14)-\mathrm{H}(16)$ & $0.94(2)$ \\
\hline $\mathrm{O}(1)-\mathrm{C}(5)$ & $1.433(2)$ & $\mathrm{C}(14)-\mathrm{H}(17)$ & $0.96(3)$ \\
\hline $\mathrm{C}(1)-\mathrm{O}(6)$ & $1.425(2)$ & $\mathrm{C}(15)-\mathrm{H}(18)$ & $0.93(3)$ \\
\hline $\mathrm{C}(1)-\mathrm{C}(2)$ & $1.511(2)$ & $\mathrm{C}(15)-\mathrm{H}(19)$ & $0.98(2)$ \\
\hline $\mathrm{C}(1)-\mathrm{H}(1)$ & $0.999(18)$ & $\mathrm{C}(15)-\mathrm{H}(20)$ & $1.01(3)$ \\
\hline $\mathrm{C}(2)-\mathrm{O}(3)$ & $1.420(2)$ & & \\
\hline$C(2)-C(3)$ & $1.517(3)$ & $\mathrm{C}(1)-\mathrm{O}(1)-\mathrm{C}(5)$ & $111.08(12)$ \\
\hline $\mathrm{C}(2)-\mathrm{H}(2)$ & $0.937(17)$ & $\mathrm{O}(1)-\mathrm{C}(1)-\mathrm{O}(6)$ & $105.85(13)$ \\
\hline $\mathrm{C}(3)-\mathrm{O}(4)$ & $1.428(2)$ & $\mathrm{O}(1)-\mathrm{C}(1)-\mathrm{C}(2)$ & $111.05(15)$ \\
\hline$C(3)-C(4)$ & $1.509(3)$ & $\mathrm{O}(6)-\mathrm{C}(1)-\mathrm{C}(2)$ & $108.31(15)$ \\
\hline $\mathrm{C}(3)-\mathrm{H}(3)$ & $0.981(19)$ & $\mathrm{O}(1)-\mathrm{C}(1)-\mathrm{H}(1)$ & $108.6(10)$ \\
\hline $\mathrm{C}(4)-\mathrm{O}(5)$ & $1.423(2)$ & $\mathrm{O}(6)-\mathrm{C}(1)-\mathrm{H}(1)$ & $110.8(10)$ \\
\hline$C(4)-C(5)$ & $1.532(2)$ & $\mathrm{C}(2)-\mathrm{C}(1)-\mathrm{H}(1)$ & $112.0(9)$ \\
\hline $\mathrm{C}(4)-\mathrm{H}(4)$ & $0.996(19)$ & $\mathrm{O}(3)-\mathrm{C}(2)-\mathrm{C}(1)$ & $112.38(14)$ \\
\hline$C(5)-C(6)$ & $1.504(3)$ & $\mathrm{O}(3)-\mathrm{C}(2)-\mathrm{C}(3)$ & $111.98(16)$ \\
\hline $\mathrm{C}(5)-\mathrm{H}(5)$ & $1.011(18)$ & $C(1)-C(2)-C(3)$ & $107.93(15)$ \\
\hline $\mathrm{C}(6)-\mathrm{O}(2)$ & $1.425(2)$ & $\mathrm{O}(3)-\mathrm{C}(2)-\mathrm{H}(2)$ & $106.6(11)$ \\
\hline $\mathrm{C}(6)-\mathrm{H}(6)$ & $0.995(19)$ & $\mathrm{C}(1)-\mathrm{C}(2)-\mathrm{H}(2)$ & $107.6(10)$ \\
\hline $\mathrm{C}(6)-\mathrm{H}(7)$ & $0.93(2)$ & $\mathrm{C}(3)-\mathrm{C}(2)-\mathrm{H}(2)$ & $110.2(10)$ \\
\hline $\mathrm{O}(2)-\mathrm{H}(8)$ & $0.81(2)$ & $\mathrm{O}(4)-\mathrm{C}(3)-\mathrm{C}(4)$ & $111.25(15)$ \\
\hline $\mathrm{O}(3)-\mathrm{H}(9)$ & $0.84(2)$ & $\mathrm{O}(4)-\mathrm{C}(3)-\mathrm{C}(2)$ & $112.83(15)$ \\
\hline $\mathrm{O}(4)-\mathrm{H}(10)$ & $0.83(2)$ & $C(4)-C(3)-C(2)$ & $110.52(14)$ \\
\hline $\mathrm{O}(5)-\mathrm{H}(11)$ & $0.83(2)$ & $\mathrm{O}(4)-\mathrm{C}(3)-\mathrm{H}(3)$ & $107.7(10)$ \\
\hline $\mathrm{O}(6)-\mathrm{C}(7)$ & $1.350(2)$ & $\mathrm{C}(4)-\mathrm{C}(3)-\mathrm{H}(3)$ & $108.1(10)$ \\
\hline $\mathrm{C}(7)-\mathrm{O}(7)$ & $1.204(2)$ & $\mathrm{C}(2)-\mathrm{C}(3)-\mathrm{H}(3)$ & $106.2(11)$ \\
\hline$C(7)-C(8)$ & $1.485(3)$ & $\mathrm{O}(5)-\mathrm{C}(4)-\mathrm{C}(3)$ & $111.62(14)$ \\
\hline$C(8)-C(9)$ & $1.393(3)$ & $\mathrm{O}(5)-\mathrm{C}(4)-\mathrm{C}(5)$ & $106.87(14)$ \\
\hline $\mathrm{C}(8)-\mathrm{C}(13)$ & $1.401(2)$ & $C(3)-C(4)-C(5)$ & $109.98(16)$ \\
\hline $\mathrm{C}(9)-\mathrm{C}(10)$ & $1.380(3)$ & $\mathrm{O}(5)-\mathrm{C}(4)-\mathrm{H}(4)$ & $111.2(11)$ \\
\hline $\mathrm{C}(9)-\mathrm{C}(14)$ & $1.512(3)$ & $\mathrm{C}(3)-\mathrm{C}(4)-\mathrm{H}(4)$ & $109.4(11)$ \\
\hline$C(10)-C(11)$ & $1.385(3)$ & $\mathrm{C}(5)-\mathrm{C}(4)-\mathrm{H}(4)$ & $107.7(10)$ \\
\hline $\mathrm{C}(10)-\mathrm{H}(12)$ & $0.95(2)$ & $\mathrm{O}(1)-\mathrm{C}(5)-\mathrm{C}(6)$ & $108.07(14)$ \\
\hline$C(11)-C(12)$ & $1.379(3)$ & $\mathrm{O}(1)-\mathrm{C}(5)-\mathrm{C}(4)$ & $109.32(14)$ \\
\hline $\mathrm{C}(11)-\mathrm{H}(13)$ & $0.91(2)$ & $C(6)-C(5)-C(4)$ & $111.42(16)$ \\
\hline $\mathrm{C}(12)-\mathrm{C}(13)$ & $1.387(3)$ & $\mathrm{O}(1)-\mathrm{C}(5)-\mathrm{H}(5)$ & $109.4(10)$ \\
\hline $\mathrm{C}(12)-\mathrm{H}(14)$ & $0.994(19)$ & $\mathrm{C}(6)-\mathrm{C}(5)-\mathrm{H}(5)$ & $109.5(9)$ \\
\hline$C(13)-C(15)$ & $1.505(3)$ & $\mathrm{C}(4)-\mathrm{C}(5)-\mathrm{H}(5)$ & 109.1(9) \\
\hline $\mathrm{C}(14)-\mathrm{H}(15)$ & $0.98(3)$ & $\mathrm{O}(2)-\mathrm{C}(6)-\mathrm{C}(5)$ & $112.23(17)$ \\
\hline
\end{tabular}




\begin{tabular}{|c|c|c|c|}
\hline $\mathrm{O}(2)-\mathrm{C}(6)-\mathrm{H}(6)$ & $112.7(9)$ & $C(12)-C(11)-C(10)$ & $119.7(2)$ \\
\hline $\mathrm{C}(5)-\mathrm{C}(6)-\mathrm{H}(6)$ & $108.4(10)$ & $\mathrm{C}(12)-\mathrm{C}(11)-\mathrm{H}(13)$ & $121.0(12)$ \\
\hline $\mathrm{O}(2)-\mathrm{C}(6)-\mathrm{H}(7)$ & $107.7(12)$ & $\mathrm{C}(10)-\mathrm{C}(11)-\mathrm{H}(13)$ & $119.2(12)$ \\
\hline $\mathrm{C}(5)-\mathrm{C}(6)-\mathrm{H}(7)$ & $108.4(11)$ & $\mathrm{C}(11)-\mathrm{C}(12)-\mathrm{C}(13)$ & $120.74(19)$ \\
\hline $\mathrm{H}(6)-\mathrm{C}(6)-\mathrm{H}(7)$ & $107.2(16)$ & $\mathrm{C}(11)-\mathrm{C}(12)-\mathrm{H}(14)$ & $122.1(12)$ \\
\hline $\mathrm{C}(6)-\mathrm{O}(2)-\mathrm{H}(8)$ & $108.6(15)$ & $\mathrm{C}(13)-\mathrm{C}(12)-\mathrm{H}(14)$ & $117.1(12)$ \\
\hline $\mathrm{C}(2)-\mathrm{O}(3)-\mathrm{H}(9)$ & $113.0(15)$ & $\mathrm{C}(12)-\mathrm{C}(13)-\mathrm{C}(8)$ & $118.50(19)$ \\
\hline $\mathrm{C}(3)-\mathrm{O}(4)-\mathrm{H}(10)$ & $107.3(14)$ & $\mathrm{C}(12)-\mathrm{C}(13)-\mathrm{C}(15)$ & $120.94(17)$ \\
\hline $\mathrm{C}(4)-\mathrm{O}(5)-\mathrm{H}(11)$ & $110.5(18)$ & $\mathrm{C}(8)-\mathrm{C}(13)-\mathrm{C}(15)$ & $120.54(18)$ \\
\hline $\mathrm{C}(7)-\mathrm{O}(6)-\mathrm{C}(1)$ & $116.98(14)$ & $\mathrm{C}(9)-\mathrm{C}(14)-\mathrm{H}(15)$ & $113.7(15)$ \\
\hline $\mathrm{O}(7)-\mathrm{C}(7)-\mathrm{O}(6)$ & $123.24(18)$ & $\mathrm{C}(9)-\mathrm{C}(14)-\mathrm{H}(16)$ & $109.5(13)$ \\
\hline $\mathrm{O}(7)-\mathrm{C}(7)-\mathrm{C}(8)$ & $123.77(18)$ & $\mathrm{H}(15)-\mathrm{C}(14)-\mathrm{H}(16)$ & $107(2)$ \\
\hline $\mathrm{O}(6)-\mathrm{C}(7)-\mathrm{C}(8)$ & $112.99(15)$ & C(9)-C(14)-H(17) & $112.4(13)$ \\
\hline $\mathrm{C}(9)-\mathrm{C}(8)-\mathrm{C}(13)$ & $121.37(18)$ & $\mathrm{H}(15)-\mathrm{C}(14)-\mathrm{H}(17)$ & 111(2) \\
\hline $\mathrm{C}(9)-\mathrm{C}(8)-\mathrm{C}(7)$ & 119.77(17) & $\mathrm{H}(16)-\mathrm{C}(14)-\mathrm{H}(17)$ & $102.5(18)$ \\
\hline$C(13)-C(8)-C(7)$ & $118.72(17)$ & $\mathrm{C}(13)-\mathrm{C}(15)-\mathrm{H}(18)$ & $112.2(16)$ \\
\hline$C(10)-C(9)-C(8)$ & $118.20(18)$ & $\mathrm{C}(13)-\mathrm{C}(15)-\mathrm{H}(19)$ & 109.3(13) \\
\hline$C(10)-C(9)-C(14)$ & $121.2(2)$ & $\mathrm{H}(18)-\mathrm{C}(15)-\mathrm{H}(19)$ & $107(2)$ \\
\hline$C(8)-C(9)-C(14)$ & $120.61(19)$ & $\mathrm{C}(13)-\mathrm{C}(15)-\mathrm{H}(20)$ & $109.6(14)$ \\
\hline$C(9)-C(10)-C(11)$ & $121.4(2)$ & $\mathrm{H}(18)-\mathrm{C}(15)-\mathrm{H}(20)$ & $105(2)$ \\
\hline $\mathrm{C}(9)-\mathrm{C}(10)-\mathrm{H}(12)$ & $119.2(14)$ & $\mathrm{H}(19)-\mathrm{C}(15)-\mathrm{H}(20)$ & $113(2)$ \\
\hline $\mathrm{C}(11)-\mathrm{C}(10)-\mathrm{H}(12)$ & $119.4(13)$ & & \\
\hline
\end{tabular}

Symmetry transformations used to generate equivalent atoms: 
Table 4. Anisotropic displacement parameters $\left(\AA^{2} \times 10^{3}\right)$ for HT1557. The anisotropic displacement factor exponent takes the form: $-2 p^{2}\left[h^{2} a^{* 2} U^{11}+\ldots+2 h k a^{*} b^{*} U^{12}\right]$

\begin{tabular}{|c|c|c|c|c|c|c|}
\hline & $\mathrm{U}^{11}$ & $\mathrm{U}^{22}$ & $\mathrm{U}^{33}$ & $\mathrm{U}^{23}$ & $\mathrm{U}^{13}$ & $\mathrm{U}^{12}$ \\
\hline $\mathrm{O}(1)$ & $16(1)$ & $17(1)$ & $20(1)$ & $3(1)$ & $0(1)$ & $1(1)$ \\
\hline $\mathrm{C}(1)$ & $21(1)$ & $15(1)$ & $18(1)$ & $-1(1)$ & $1(1)$ & $1(1)$ \\
\hline $\mathrm{C}(2)$ & $16(1)$ & $18(1)$ & $20(1)$ & $-1(1)$ & $6(1)$ & $0(1)$ \\
\hline $\mathrm{C}(3)$ & $15(1)$ & $17(1)$ & $23(1)$ & $-3(1)$ & $-3(1)$ & $1(1)$ \\
\hline$C(4)$ & $19(1)$ & $14(1)$ & $22(1)$ & $2(1)$ & $-4(1)$ & $2(1)$ \\
\hline$C(5)$ & $19(1)$ & $16(1)$ & $20(1)$ & $4(1)$ & $-4(1)$ & $0(1)$ \\
\hline$C(6)$ & $16(1)$ & $26(1)$ & $23(1)$ & $4(1)$ & $-1(1)$ & $-2(1)$ \\
\hline $\mathrm{O}(2)$ & $16(1)$ & $25(1)$ & $31(1)$ & $7(1)$ & $-1(1)$ & $-1(1)$ \\
\hline $\mathrm{O}(3)$ & $17(1)$ & $18(1)$ & $22(1)$ & $-2(1)$ & $1(1)$ & $-1(1)$ \\
\hline $\mathrm{O}(4)$ & $14(1)$ & $20(1)$ & $36(1)$ & $0(1)$ & $-3(1)$ & $-1(1)$ \\
\hline $\mathrm{O}(5)$ & $19(1)$ & $21(1)$ & $31(1)$ & $9(1)$ & $-5(1)$ & $-1(1)$ \\
\hline $\mathrm{O}(6)$ & $24(1)$ & $18(1)$ & $17(1)$ & $1(1)$ & $-4(1)$ & $-1(1)$ \\
\hline$C(7)$ & $22(1)$ & $22(1)$ & $20(1)$ & $-2(1)$ & $1(1)$ & $-4(1)$ \\
\hline $\mathrm{O}(7)$ & $49(1)$ & $20(1)$ & $31(1)$ & $-1(1)$ & $-16(1)$ & $-3(1)$ \\
\hline$C(8)$ & $25(1)$ & $20(1)$ & $15(1)$ & $-1(1)$ & $-5(1)$ & $-6(1)$ \\
\hline$C(9)$ & $30(1)$ & $21(1)$ & $20(1)$ & $0(1)$ & $-2(1)$ & $-5(1)$ \\
\hline$C(10)$ & $46(1)$ & $25(1)$ & $19(1)$ & $3(1)$ & $-2(1)$ & $-8(1)$ \\
\hline$C(11)$ & $43(1)$ & $21(1)$ & $26(1)$ & $4(1)$ & $-10(1)$ & $2(1)$ \\
\hline$C(12)$ & $32(1)$ & $22(1)$ & $31(1)$ & $-1(1)$ & $-2(1)$ & $0(1)$ \\
\hline$C(13)$ & $27(1)$ & $20(1)$ & $23(1)$ & $-1(1)$ & $-2(1)$ & $-5(1)$ \\
\hline$C(14)$ & $34(1)$ & $37(1)$ & $32(1)$ & $2(1)$ & $9(1)$ & $-2(1)$ \\
\hline$C(15)$ & $31(1)$ & $29(1)$ & $35(2)$ & $2(1)$ & $8(1)$ & $2(1)$ \\
\hline
\end{tabular}


Table 5. Hydrogen coordinates $\left(\mathrm{x} 10^{4}\right)$ and isotropic displacement parameters $\left(\AA^{2} \times 10^{3}\right)$ for HT1557.

\begin{tabular}{|c|c|c|c|c|}
\hline & $\mathrm{x}$ & $\mathrm{y}$ & $\mathrm{z}$ & $\mathrm{U}(\mathrm{eq})$ \\
\hline $\mathrm{H}(1)$ & 1995(19) & $1900(20)$ & $5918(8)$ & $9(4)$ \\
\hline $\mathrm{H}(2)$ & $4340(20)$ & $3430(19)$ & $6058(9)$ & $10(4)$ \\
\hline $\mathrm{H}(3)$ & $4390(20)$ & $1220(20)$ & $6595(9)$ & $15(5)$ \\
\hline $\mathrm{H}(4)$ & $2880(20)$ & $2720(20)$ & $7644(9)$ & $22(5)$ \\
\hline $\mathrm{H}(5)$ & $1412(18)$ & $490(20)$ & $6819(8)$ & $10(4)$ \\
\hline $\mathrm{H}(6)$ & $-226(19)$ & $2210(20)$ & $7790(8)$ & $13(4)$ \\
\hline $\mathrm{H}(7)$ & $430(20)$ & $600(20)$ & $7900(10)$ & $24(5)$ \\
\hline $\mathrm{H}(8)$ & $-2070(30)$ & $1190(20)$ & $7189(11)$ & $33(7)$ \\
\hline $\mathrm{H}(9)$ & $2830(30)$ & $4820(20)$ & $6989(10)$ & $35(7)$ \\
\hline $\mathrm{H}(10)$ & $5900(30)$ & $3370(30)$ & $7192(11)$ & $35(7)$ \\
\hline $\mathrm{H}(11)$ & $4460(30)$ & $650(30)$ & $7883(12)$ & $51(8)$ \\
\hline $\mathrm{H}(12)$ & $490(30)$ & $6990(30)$ & $3662(12)$ & $43(7)$ \\
\hline $\mathrm{H}(13)$ & $-2120(20)$ & $7900(20)$ & 3914(9) & $27(6)$ \\
\hline $\mathrm{H}(14)$ & $-3610(20)$ & $7070(20)$ & $4829(10)$ & $33(6)$ \\
\hline $\mathrm{H}(15)$ & $2360(30)$ & $3810(30)$ & $4134(13)$ & $59(8)$ \\
\hline $\mathrm{H}(16)$ & $3050(30)$ & $5040(30)$ & $4578(11)$ & $39(7)$ \\
\hline $\mathrm{H}(17)$ & $2880(30)$ & $5440(30)$ & $3895(11)$ & $46(7)$ \\
\hline $\mathrm{H}(18)$ & $-2640(30)$ & $3990(30)$ & $5725(12)$ & $52(8)$ \\
\hline $\mathrm{H}(19)$ & $-3890(20)$ & $5280(20)$ & $5644(10)$ & $32(6)$ \\
\hline $\mathrm{H}(20)$ & $-2130(30)$ & $5460(30)$ & $6058(12)$ & $55(8)$ \\
\hline
\end{tabular}


Table 6. Torsion angles $\left[^{\circ}\right]$ for HT1557.

\begin{tabular}{|c|c|c|c|}
\hline $\mathrm{C}(5)-\mathrm{O}(1)-\mathrm{C}(1)-\mathrm{O}(6)$ & $177.86(13)$ & $\mathrm{O}(1)-\mathrm{C}(1)-\mathrm{O}(6)-\mathrm{C}(7)$ & $-98.15(17)$ \\
\hline $\mathrm{C}(5)-\mathrm{O}(1)-\mathrm{C}(1)-\mathrm{C}(2)$ & $-64.80(18)$ & $\mathrm{C}(2)-\mathrm{C}(1)-\mathrm{O}(6)-\mathrm{C}(7)$ & $142.69(15)$ \\
\hline $\mathrm{O}(1)-\mathrm{C}(1)-\mathrm{C}(2)-\mathrm{O}(3)$ & $-64.3(2)$ & $\mathrm{C}(1)-\mathrm{O}(6)-\mathrm{C}(7)-\mathrm{O}(7)$ & $-0.1(2)$ \\
\hline $\mathrm{O}(6)-\mathrm{C}(1)-\mathrm{C}(2)-\mathrm{O}(3)$ & $51.6(2)$ & $\mathrm{C}(1)-\mathrm{O}(6)-\mathrm{C}(7)-\mathrm{C}(8)$ & 179.92(14) \\
\hline $\mathrm{O}(1)-\mathrm{C}(1)-\mathrm{C}(2)-\mathrm{C}(3)$ & $59.72(19)$ & $\mathrm{O}(7)-\mathrm{C}(7)-\mathrm{C}(8)-\mathrm{C}(9)$ & $-83.4(2)$ \\
\hline $\mathrm{O}(6)-\mathrm{C}(1)-\mathrm{C}(2)-\mathrm{C}(3)$ & $175.54(15)$ & $\mathrm{O}(6)-\mathrm{C}(7)-\mathrm{C}(8)-\mathrm{C}(9)$ & $96.6(2)$ \\
\hline $\mathrm{O}(3)-\mathrm{C}(2)-\mathrm{C}(3)-\mathrm{O}(4)$ & $-55.77(19)$ & $\mathrm{O}(7)-\mathrm{C}(7)-\mathrm{C}(8)-\mathrm{C}(13)$ & $92.4(2)$ \\
\hline $\mathrm{C}(1)-\mathrm{C}(2)-\mathrm{C}(3)-\mathrm{O}(4)$ & $-179.98(15)$ & $\mathrm{O}(6)-\mathrm{C}(7)-\mathrm{C}(8)-\mathrm{C}(13)$ & $-87.65(19)$ \\
\hline $\mathrm{O}(3)-\mathrm{C}(2)-\mathrm{C}(3)-\mathrm{C}(4)$ & $69.50(19)$ & $\mathrm{C}(13)-\mathrm{C}(8)-\mathrm{C}(9)-\mathrm{C}(10)$ & $-2.2(3)$ \\
\hline $\mathrm{C}(1)-\mathrm{C}(2)-\mathrm{C}(3)-\mathrm{C}(4)$ & $-54.7(2)$ & $\mathrm{C}(7)-\mathrm{C}(8)-\mathrm{C}(9)-\mathrm{C}(10)$ & $173.51(18)$ \\
\hline $\mathrm{O}(4)-\mathrm{C}(3)-\mathrm{C}(4)-\mathrm{O}(5)$ & $-61.22(19)$ & $\mathrm{C}(13)-\mathrm{C}(8)-\mathrm{C}(9)-\mathrm{C}(14)$ & $176.54(19)$ \\
\hline $\mathrm{C}(2)-\mathrm{C}(3)-\mathrm{C}(4)-\mathrm{O}(5)$ & $172.62(15)$ & $C(7)-C(8)-C(9)-C(14)$ & $-7.8(3)$ \\
\hline $\mathrm{O}(4)-\mathrm{C}(3)-\mathrm{C}(4)-\mathrm{C}(5)$ & $-179.67(15)$ & C(8)-C(9)-C(10)-C(11) & $-0.1(3)$ \\
\hline$C(2)-C(3)-C(4)-C(5)$ & $54.2(2)$ & $\mathrm{C}(14)-\mathrm{C}(9)-\mathrm{C}(10)-\mathrm{C}(11)$ & $-178.9(2)$ \\
\hline $\mathrm{C}(1)-\mathrm{O}(1)-\mathrm{C}(5)-\mathrm{C}(6)$ & $-176.69(14)$ & $\mathrm{C}(9)-\mathrm{C}(10)-\mathrm{C}(11)-\mathrm{C}(12)$ & $1.6(3)$ \\
\hline $\mathrm{C}(1)-\mathrm{O}(1)-\mathrm{C}(5)-\mathrm{C}(4)$ & $61.87(19)$ & $\mathrm{C}(10)-\mathrm{C}(11)-\mathrm{C}(12)-\mathrm{C}(13)$ & $-0.7(3)$ \\
\hline $\mathrm{O}(5)-\mathrm{C}(4)-\mathrm{C}(5)-\mathrm{O}(1)$ & $-177.77(15)$ & $\mathrm{C}(11)-\mathrm{C}(12)-\mathrm{C}(13)-\mathrm{C}(8)$ & $-1.5(3)$ \\
\hline $\mathrm{C}(3)-\mathrm{C}(4)-\mathrm{C}(5)-\mathrm{O}(1)$ & $-56.44(19)$ & $\mathrm{C}(11)-\mathrm{C}(12)-\mathrm{C}(13)-\mathrm{C}(15)$ & $176.8(2)$ \\
\hline $\mathrm{O}(5)-\mathrm{C}(4)-\mathrm{C}(5)-\mathrm{C}(6)$ & $62.8(2)$ & $\mathrm{C}(9)-\mathrm{C}(8)-\mathrm{C}(13)-\mathrm{C}(12)$ & $3.0(3)$ \\
\hline$C(3)-C(4)-C(5)-C(6)$ & $-175.82(15)$ & $\mathrm{C}(7)-\mathrm{C}(8)-\mathrm{C}(13)-\mathrm{C}(12)$ & $-172.73(17)$ \\
\hline $\mathrm{O}(1)-\mathrm{C}(5)-\mathrm{C}(6)-\mathrm{O}(2)$ & $73.8(2)$ & $\mathrm{C}(9)-\mathrm{C}(8)-\mathrm{C}(13)-\mathrm{C}(15)$ & $-175.34(19)$ \\
\hline $\mathrm{C}(4)-\mathrm{C}(5)-\mathrm{C}(6)-\mathrm{O}(2)$ & $-166.05(15)$ & $\mathrm{C}(7)-\mathrm{C}(8)-\mathrm{C}(13)-\mathrm{C}(15)$ & $8.9(3)$ \\
\hline
\end{tabular}

Symmetry transformations used to generate equivalent atoms: 


\section{REFERENCES}

1. S. Zheng, L. Laraia, C. J. O’ Connor, D. Sorrell, Y. S. Tan, Z. Xu, A. R. Venkitaraman, W. Wu, D. R. Spring, Org. Biomol. Chem. 2012, 10, 2590.

2. J. Atena, D. Mahdieh, A. Maliheh, A. Amir, S. Ali, S. Hadi, M. Jebraeel, S. Hamid, Bioorg. Med. Chem. 2012, 20, 5518.

3. B. Schmidt, M. Riemer, M. Karras, J. Org. Chem. 2013, 78, 8680.

4. T. Fukuyama, M. Cheung, T. Kan, Synlett 1999, 8, 1301.

5. H. Jacin, J. M. Slanski, R. J. Moshy, J. Chromatogr. 1968, 37, 103.

6. T. Fujita, K. Ohira, K. Miyatake, Y. Nakano, M. Nakayama, Chem. Pharm. Bull. 1995, 43, 920.

7. K. P. Latté, H. Kolodziej, Phytochemistry 2000, 54, 701.

8. M. Huang, G. E. Garrett, N. Birlirakis, L. Bóhe, D. A. Pratt, D. Crich, Nature Chem. 2012, 4, 663.

9. D. A. Singleton, A. A. Thomas, J. Am. Chem. Soc. 1995, 117, 9357.

10. J. K. Lee, A. D. Bain, P. J. Berti, J. Am. Chem. Soc. 2004, 126, 3769.

11. Z. Otwinoski, W. Minor, Methods in Enzymol. 1997, 276, 307.

12. M. C. Burla, R. Caliandro, M. Camalli, B. Carrozzini, G. L. Cascarano, L. De Caro, C. Giacovazzo, G. Polidori, R. Spagna, J. Appl. Cryst. 2005, 38, 381.

13. R. H. Blessing, Acta Crystallogr. Sect. A 1995, A51, 33.

14. G. M. Sheldrick, Acta Crystallogr. Sect. A 2008, A64, 112. 
VIII. 'H-NMR and ${ }^{1} \mathrm{C}-\mathrm{NMR}$ SPECTRA REPRINTS

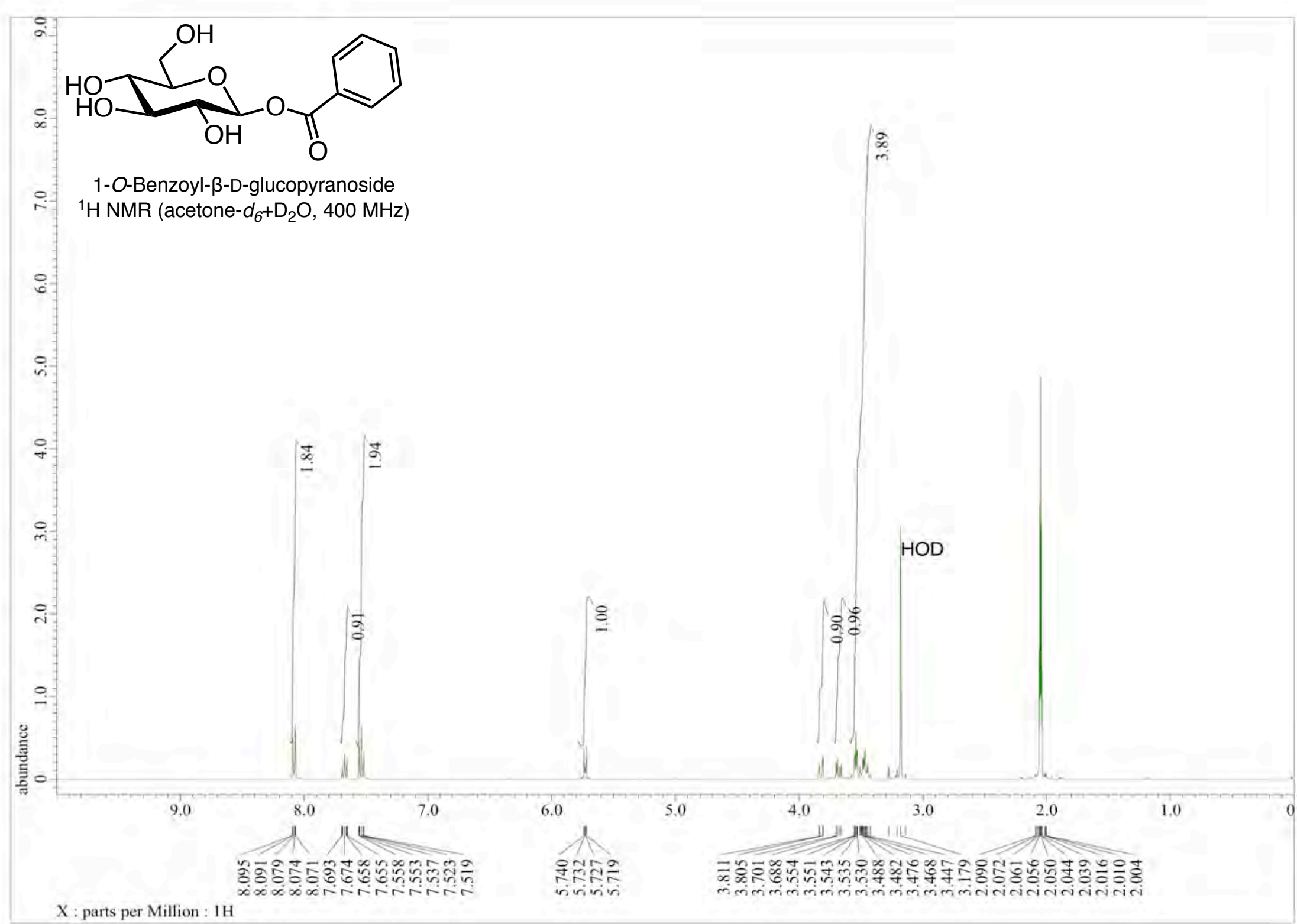




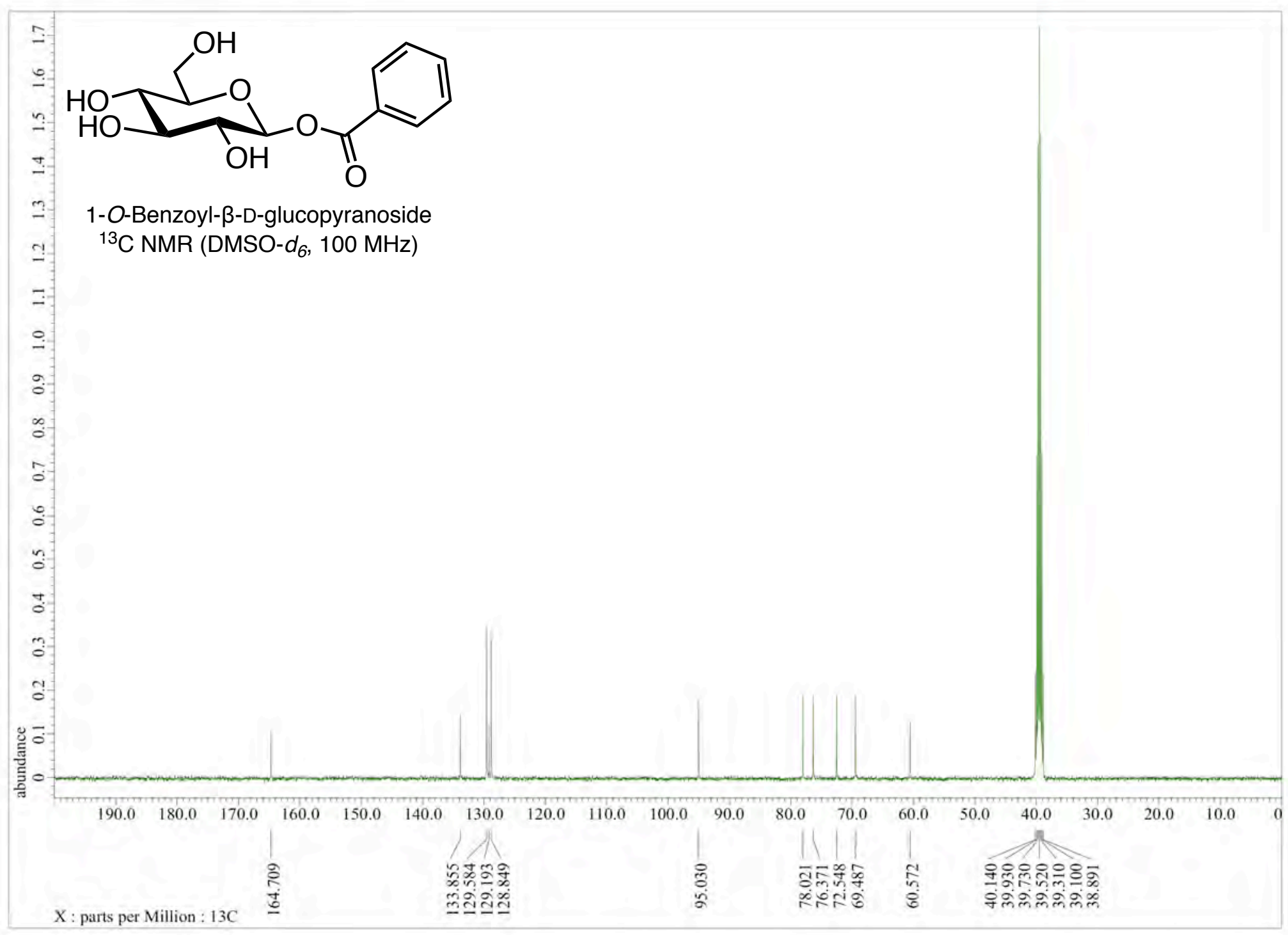




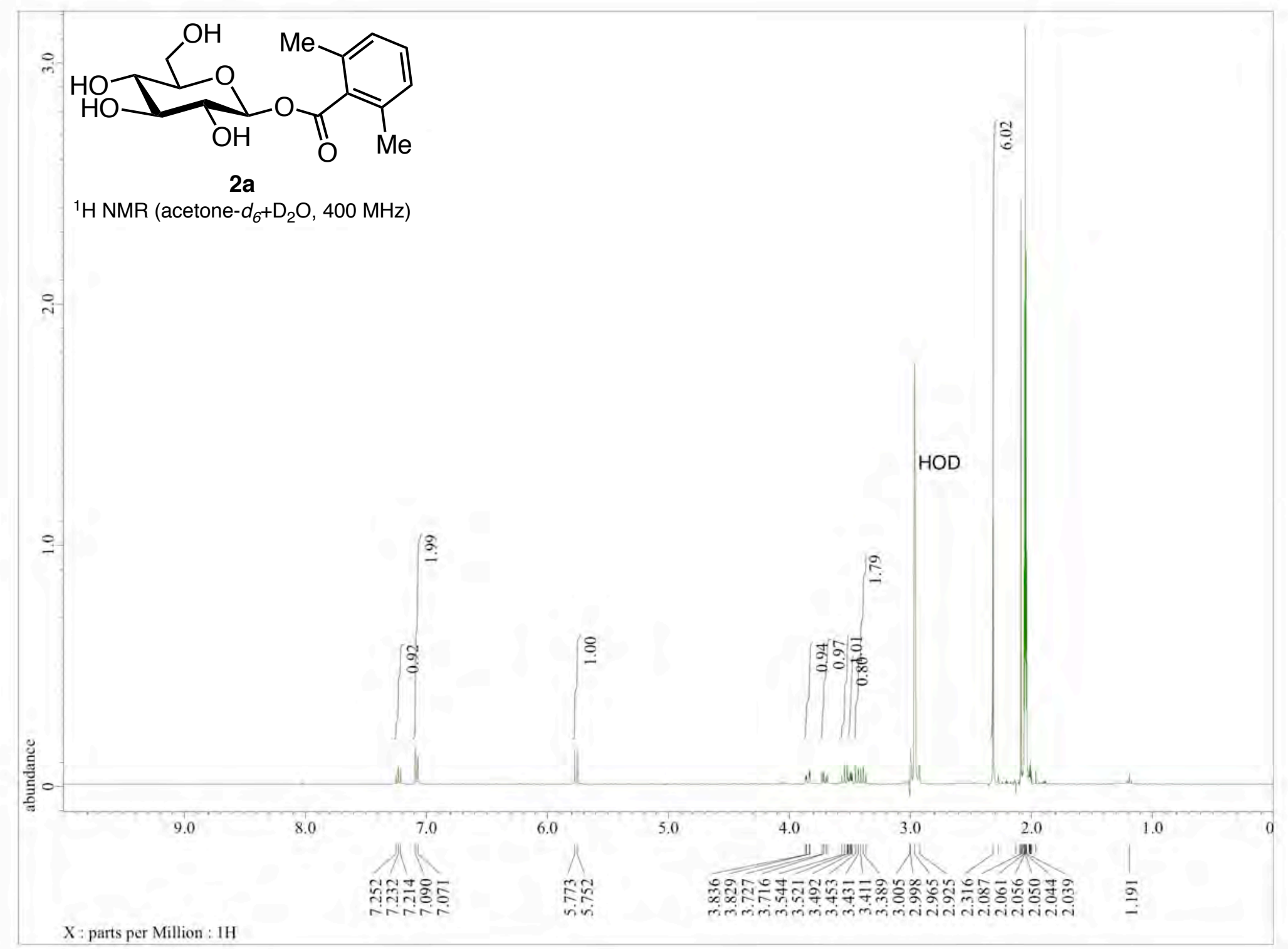




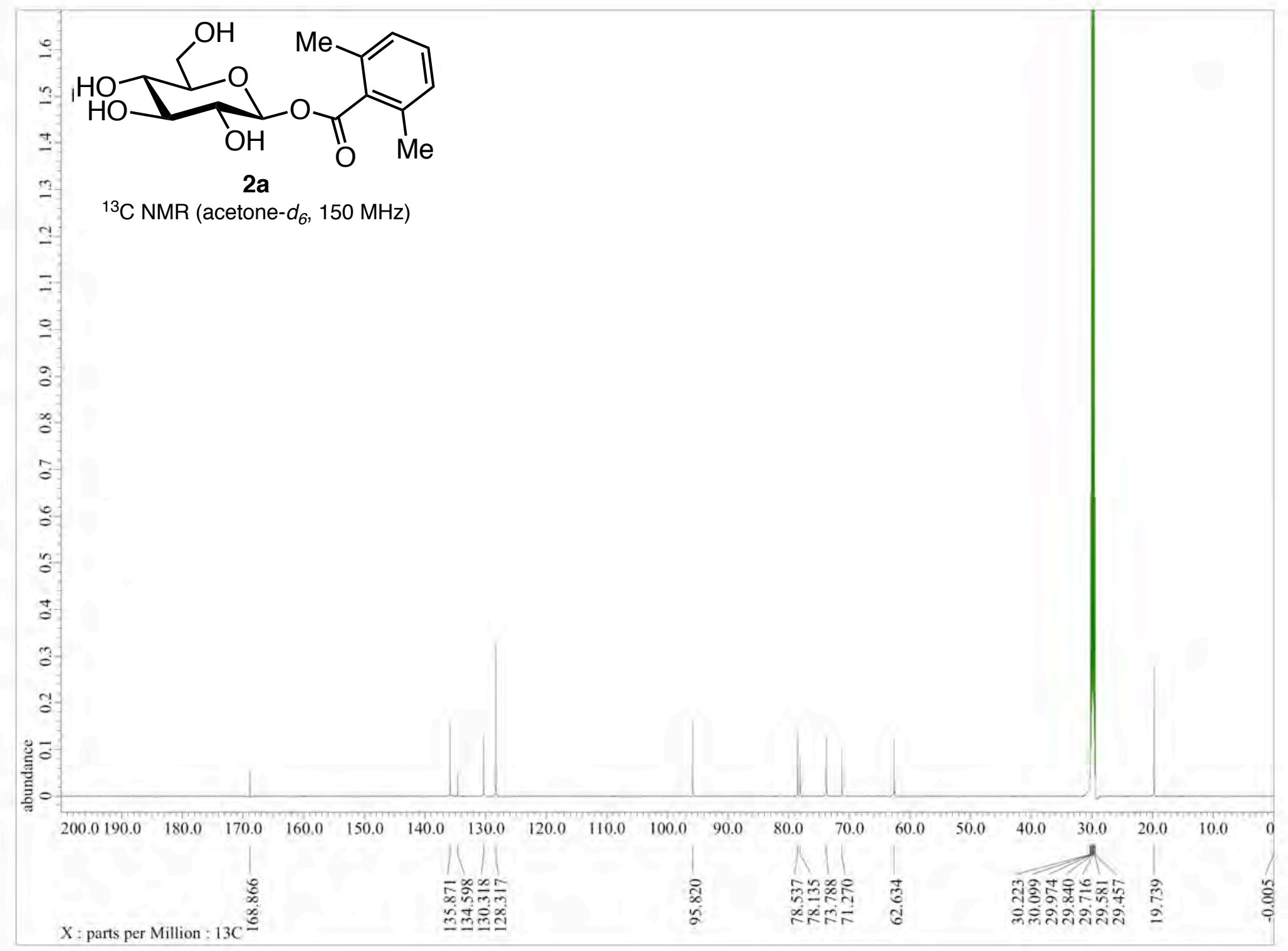




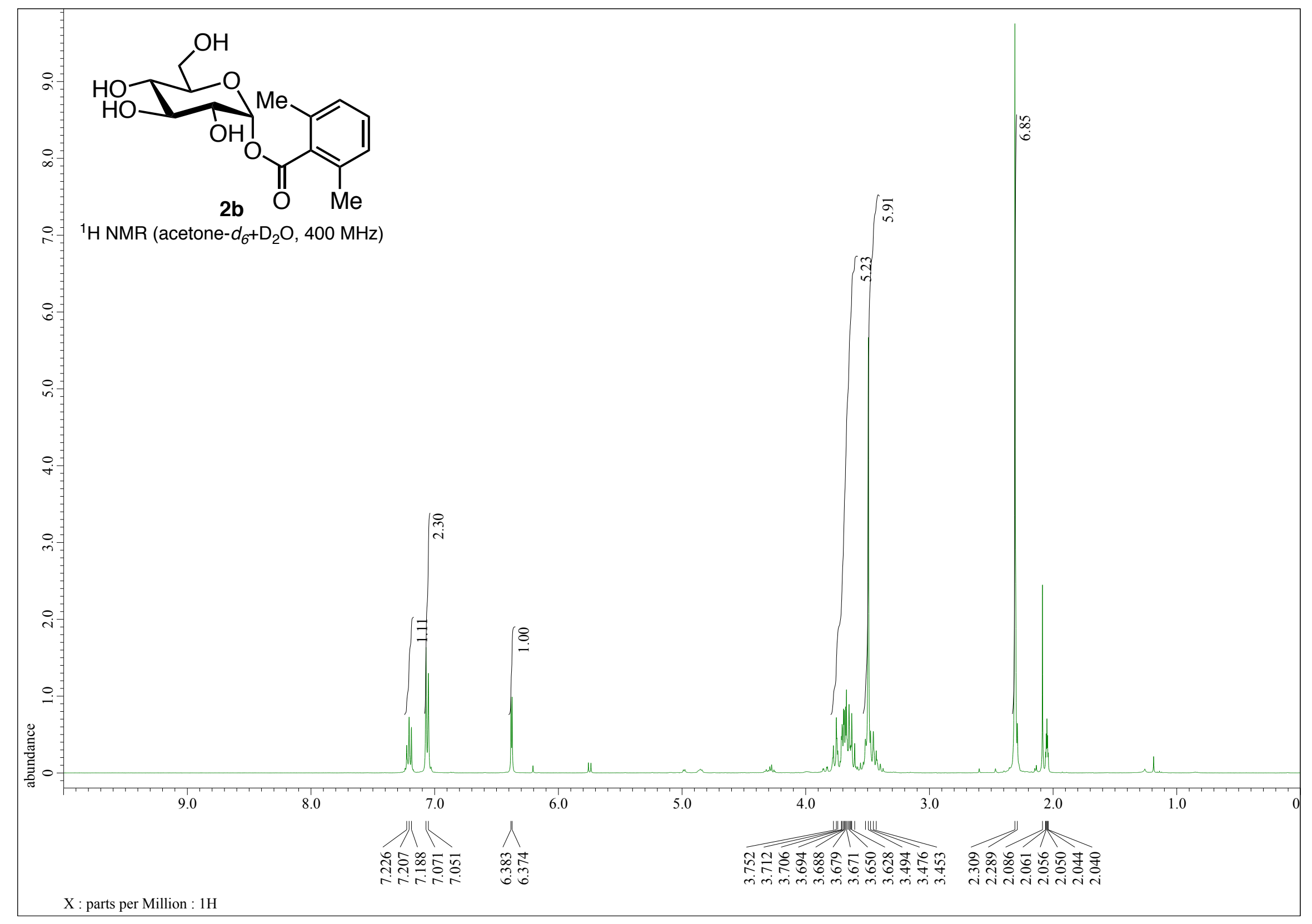




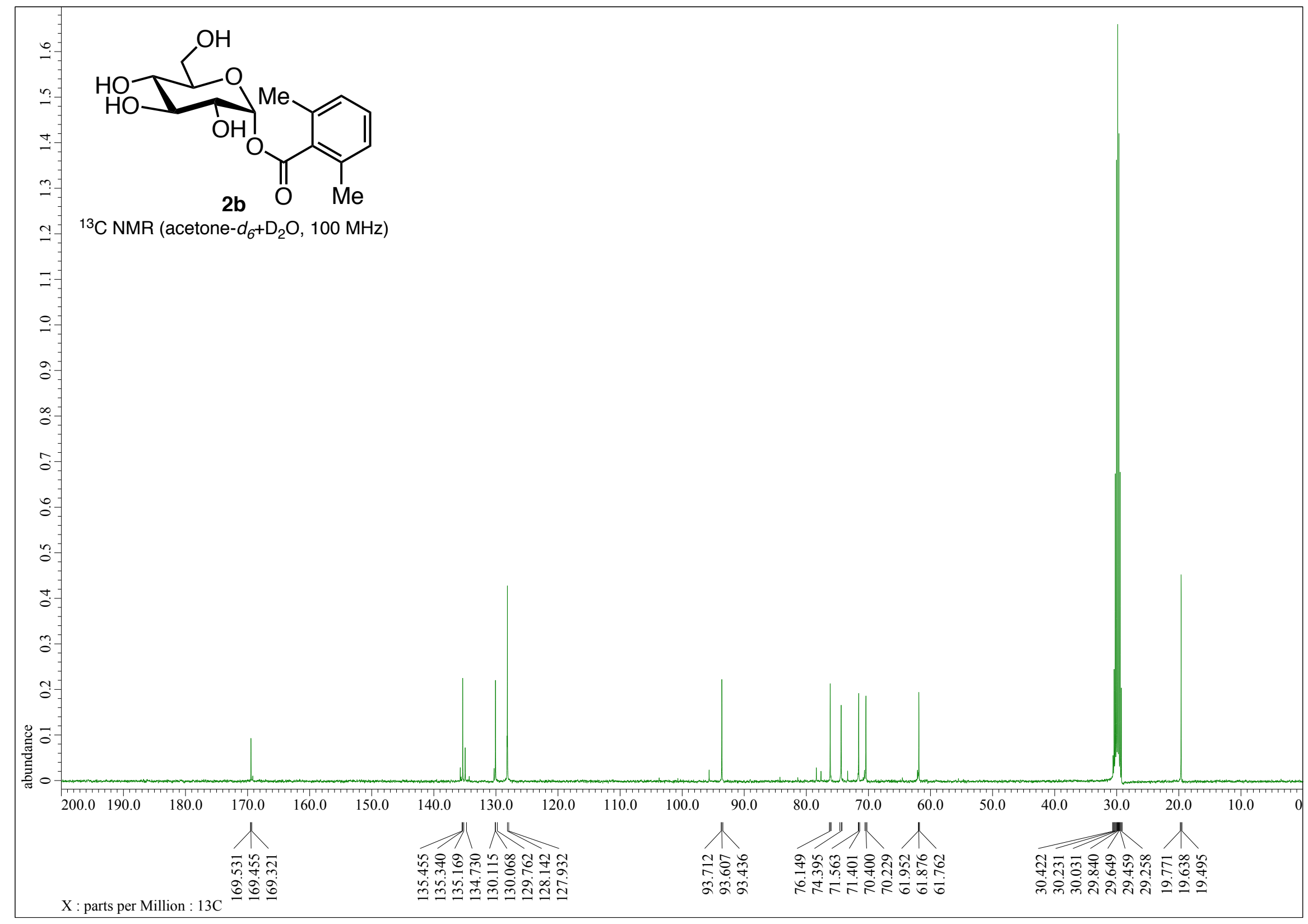




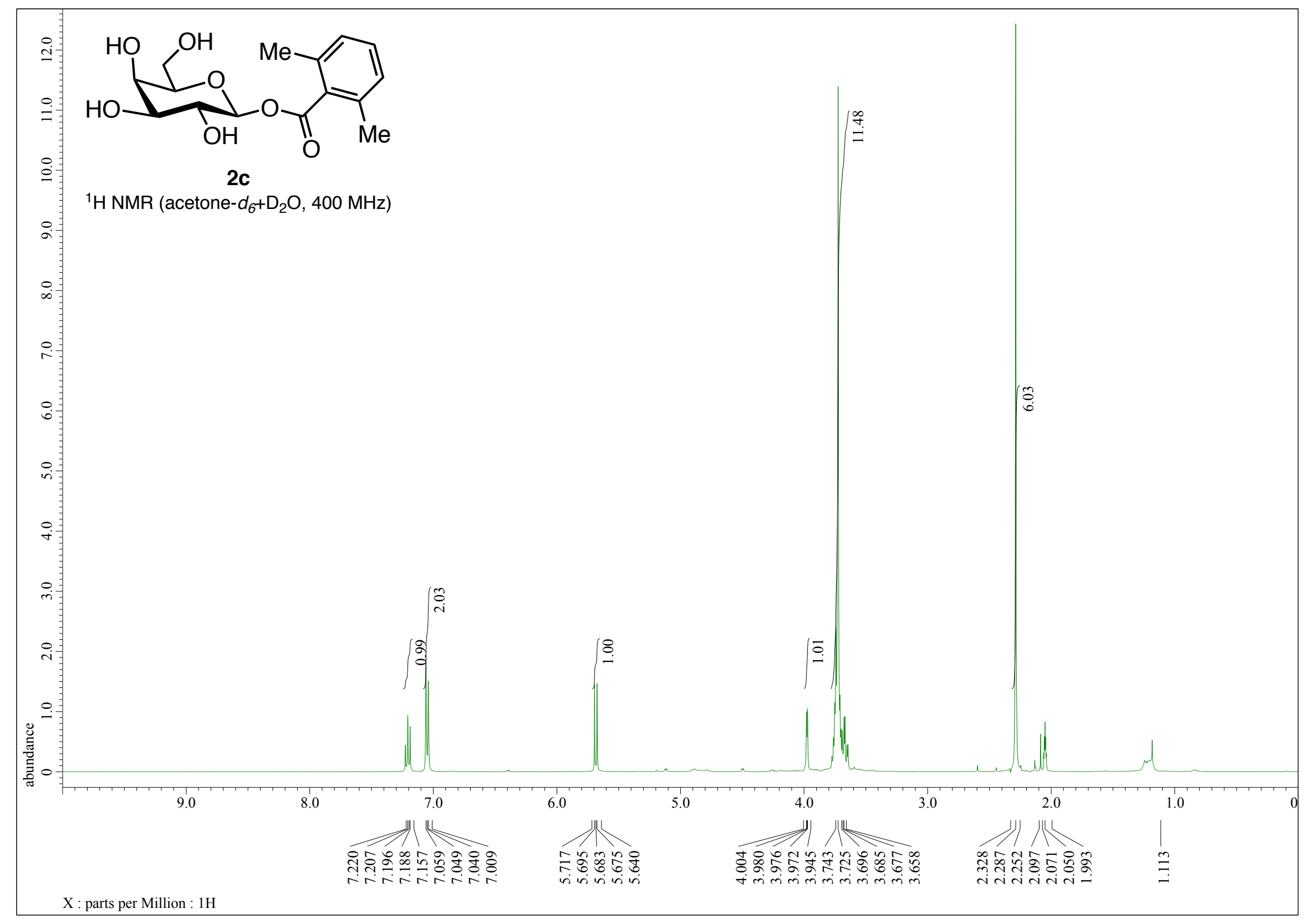




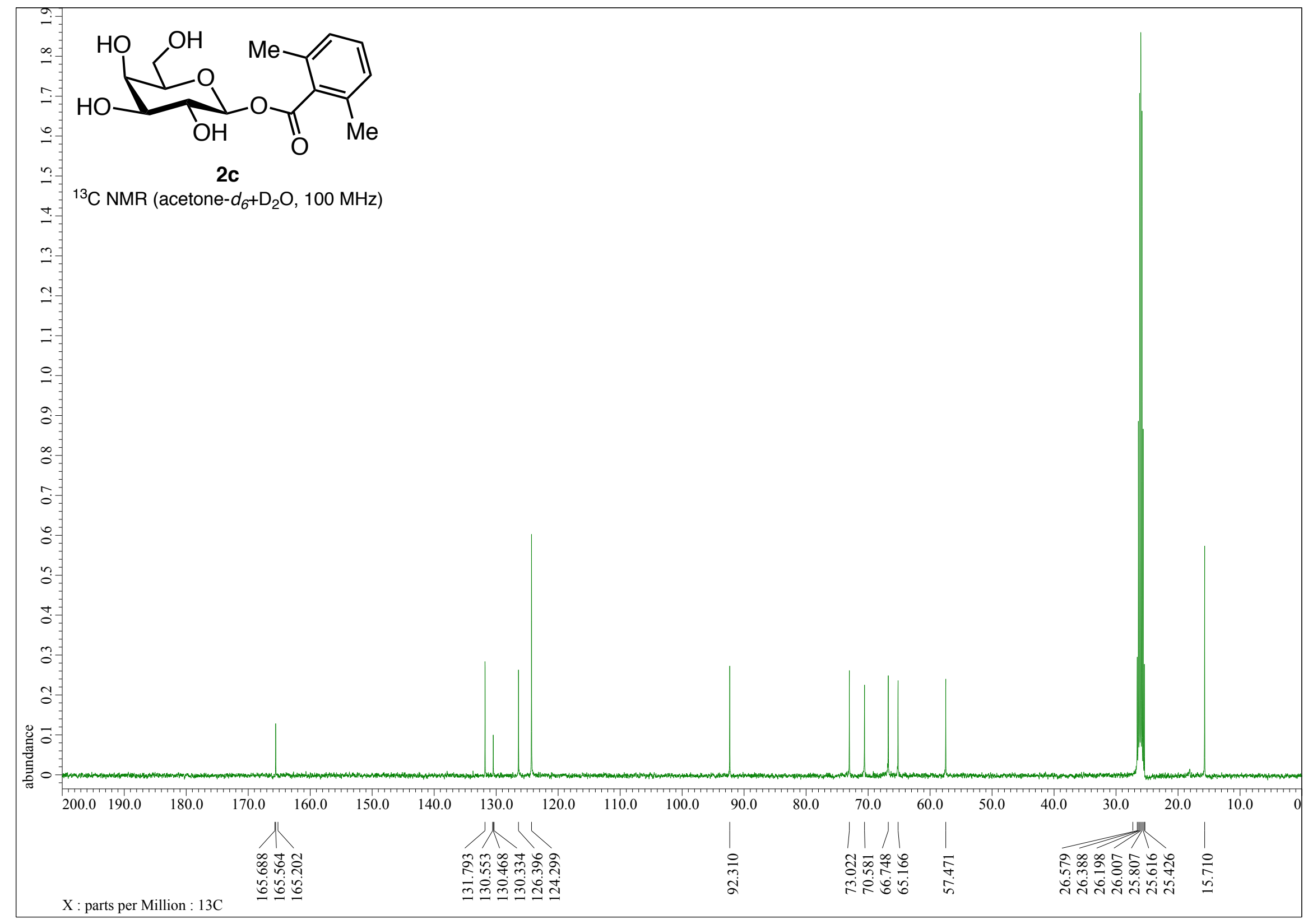




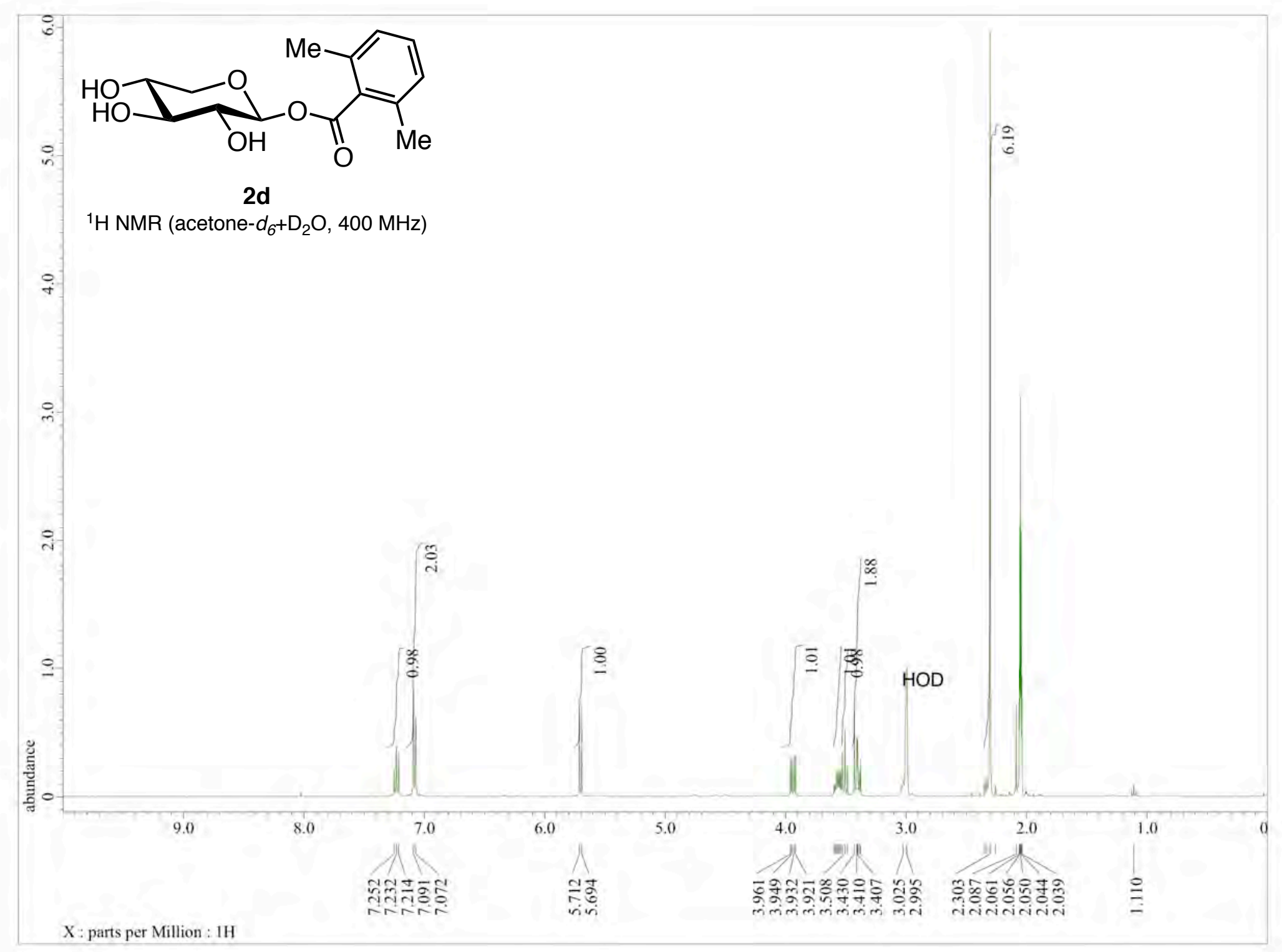




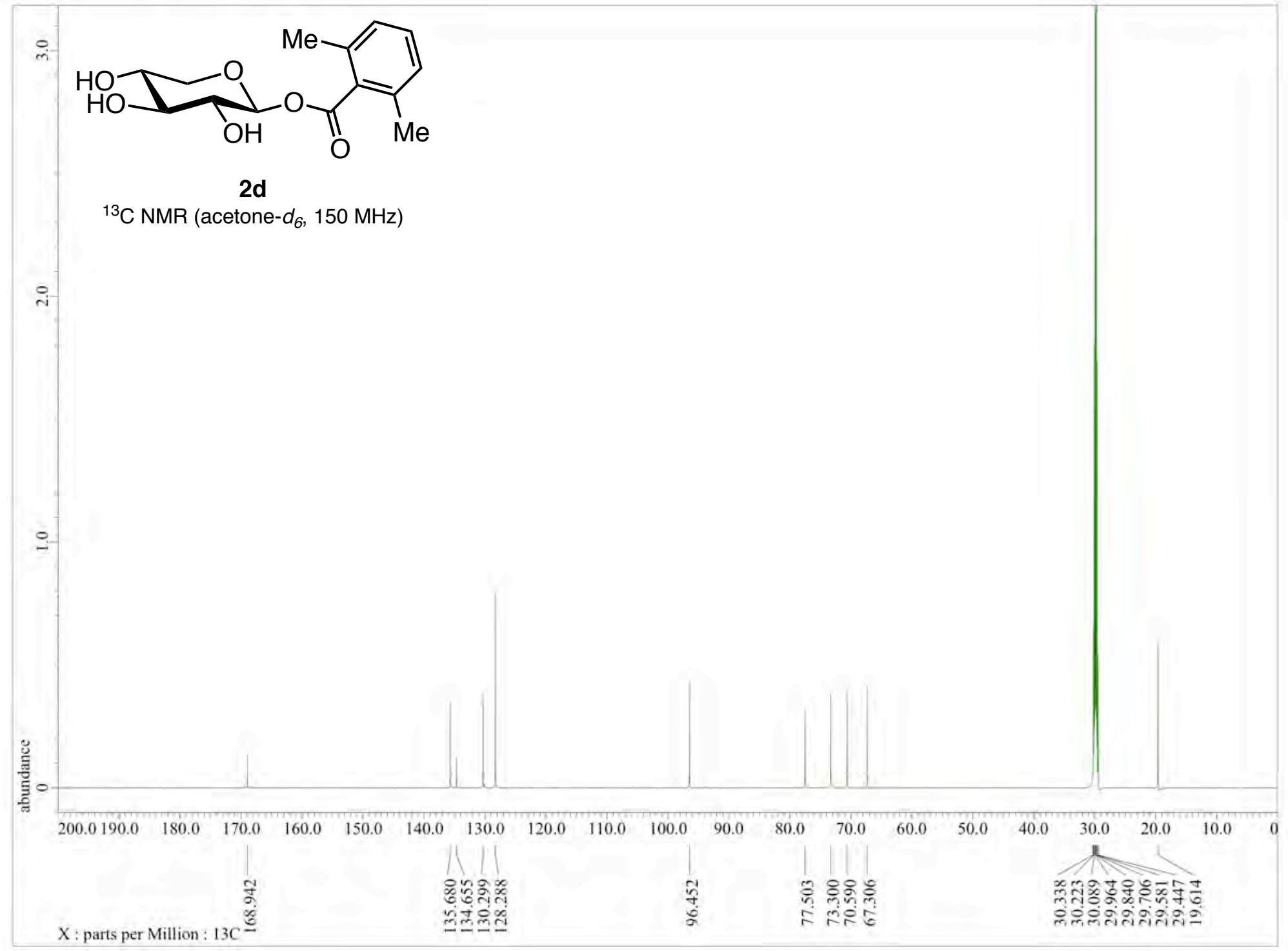




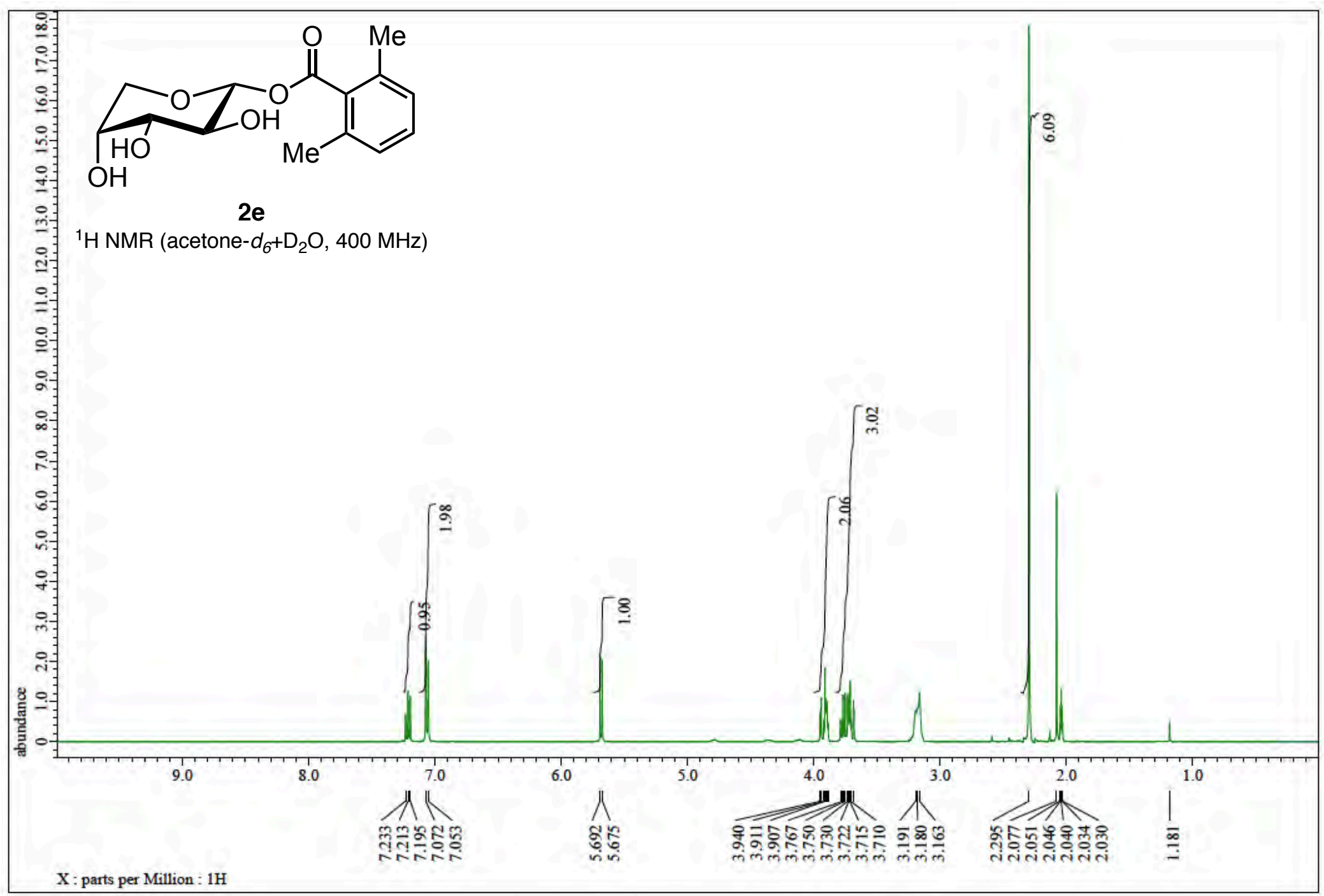




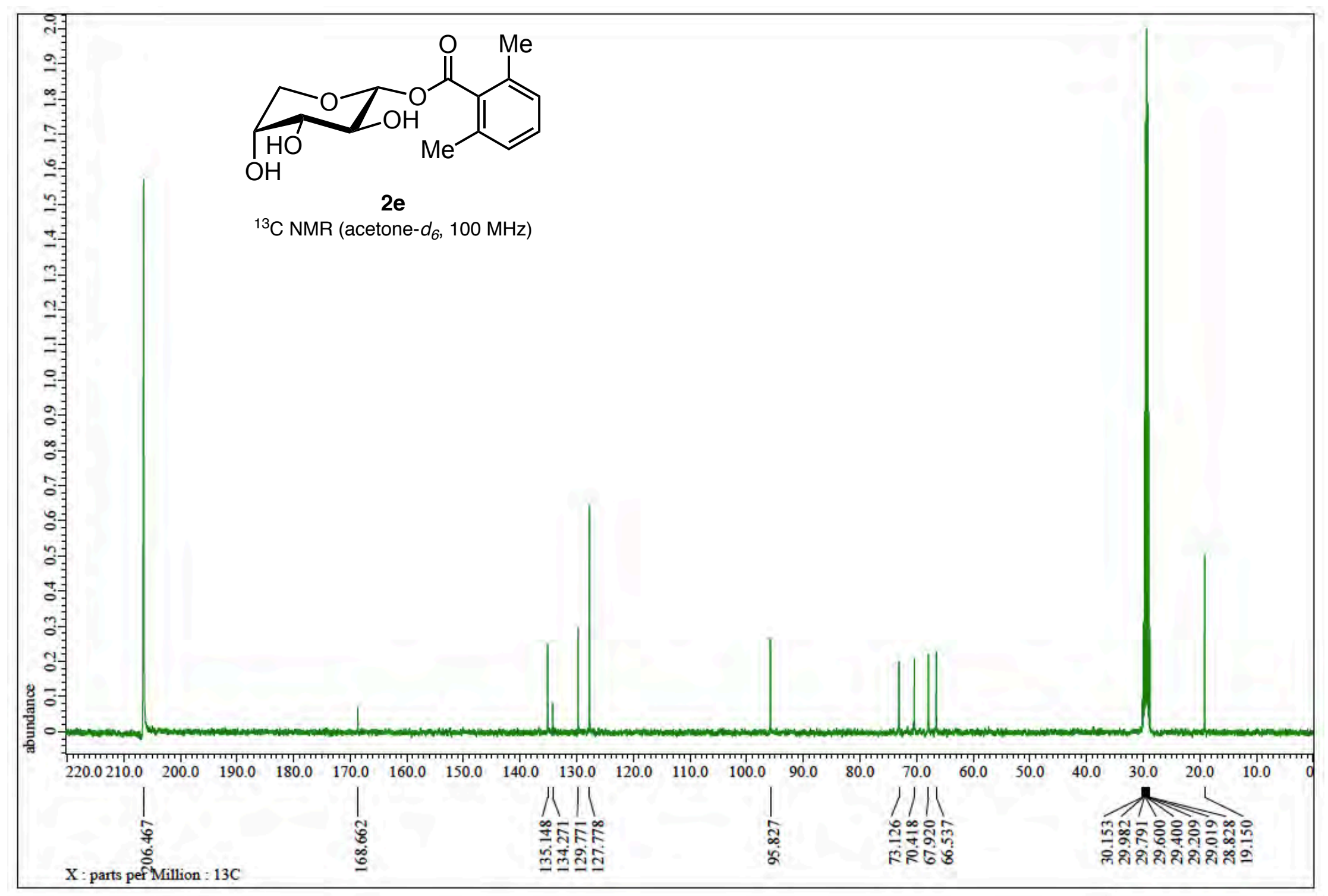




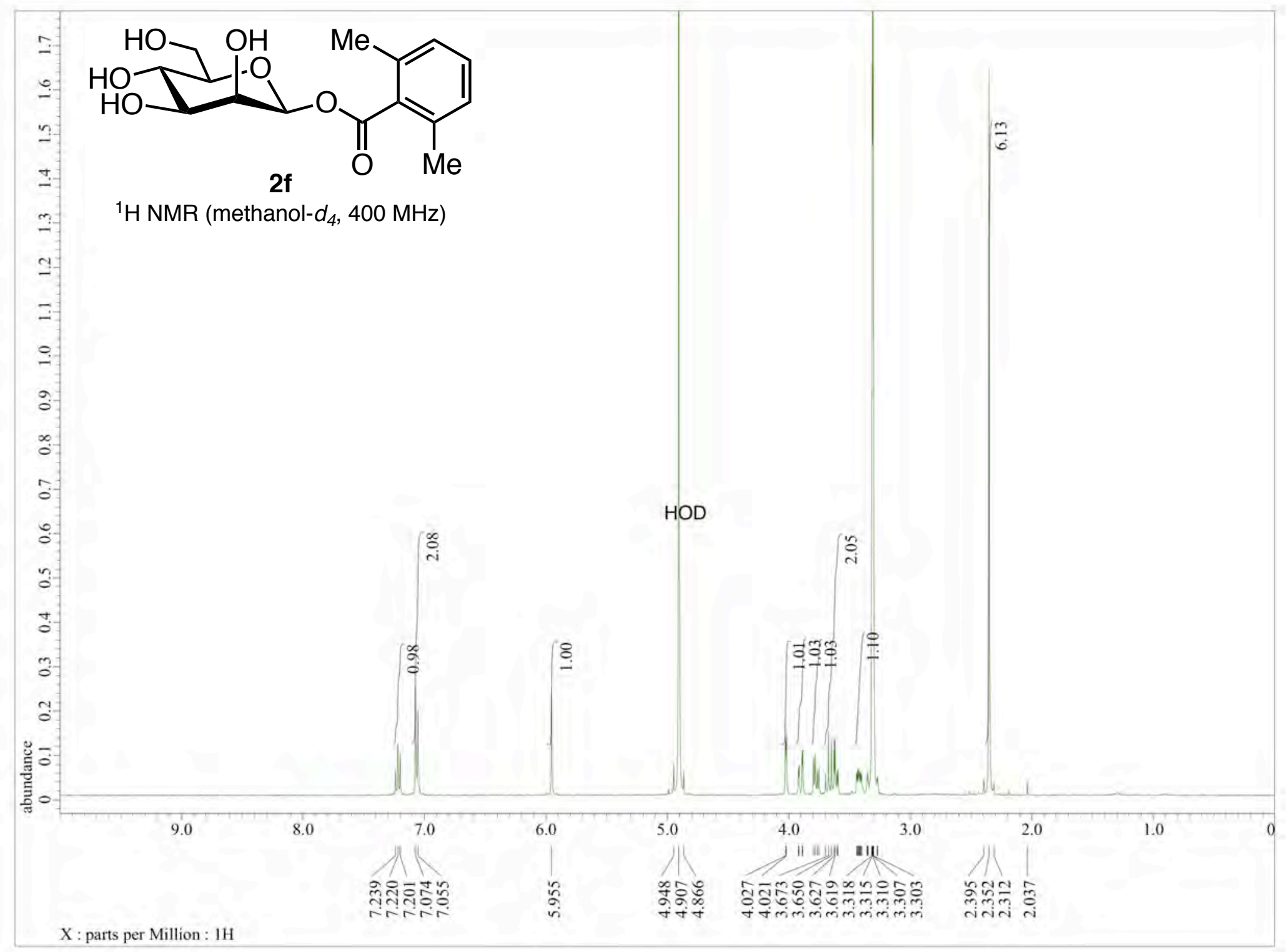




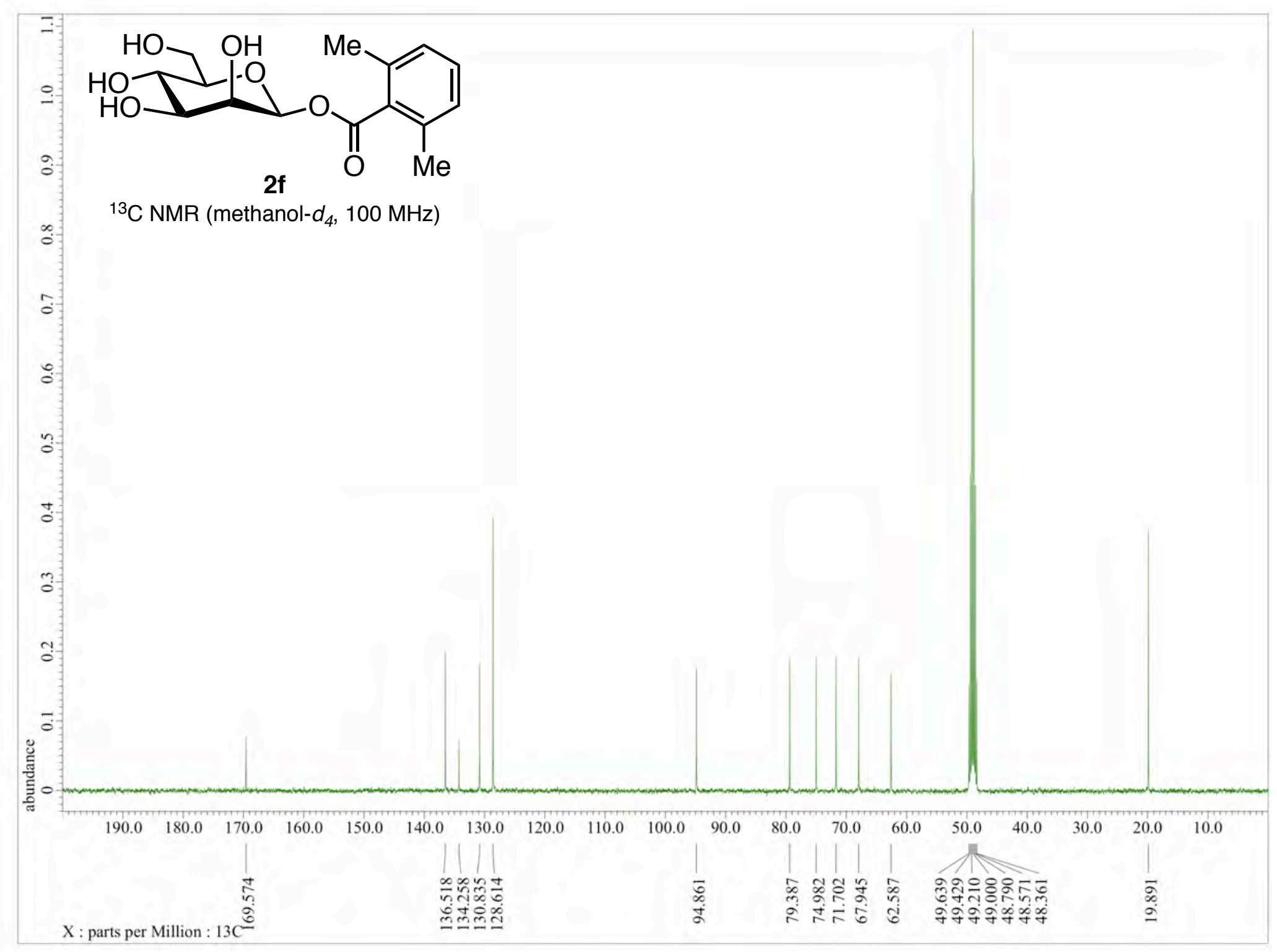




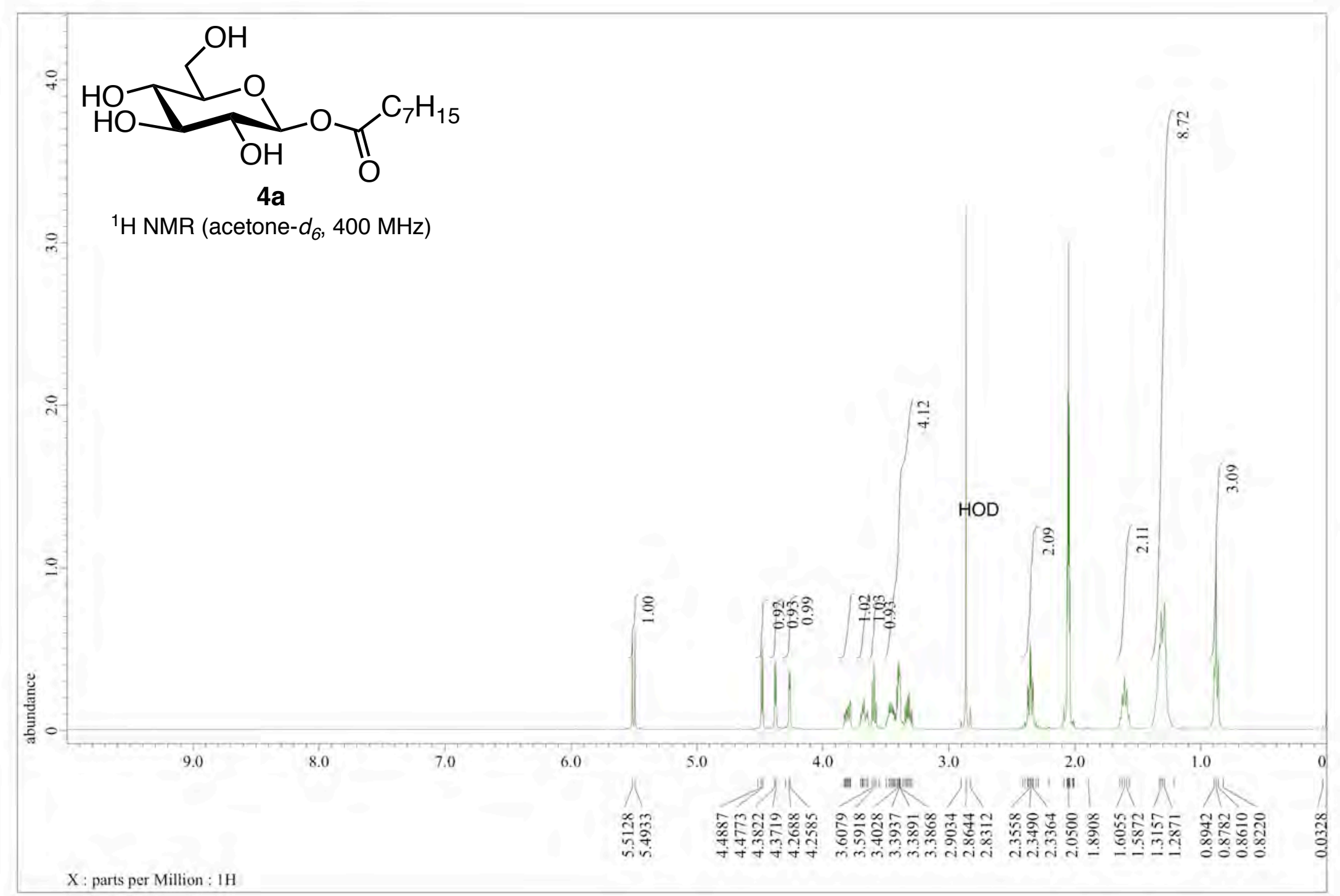




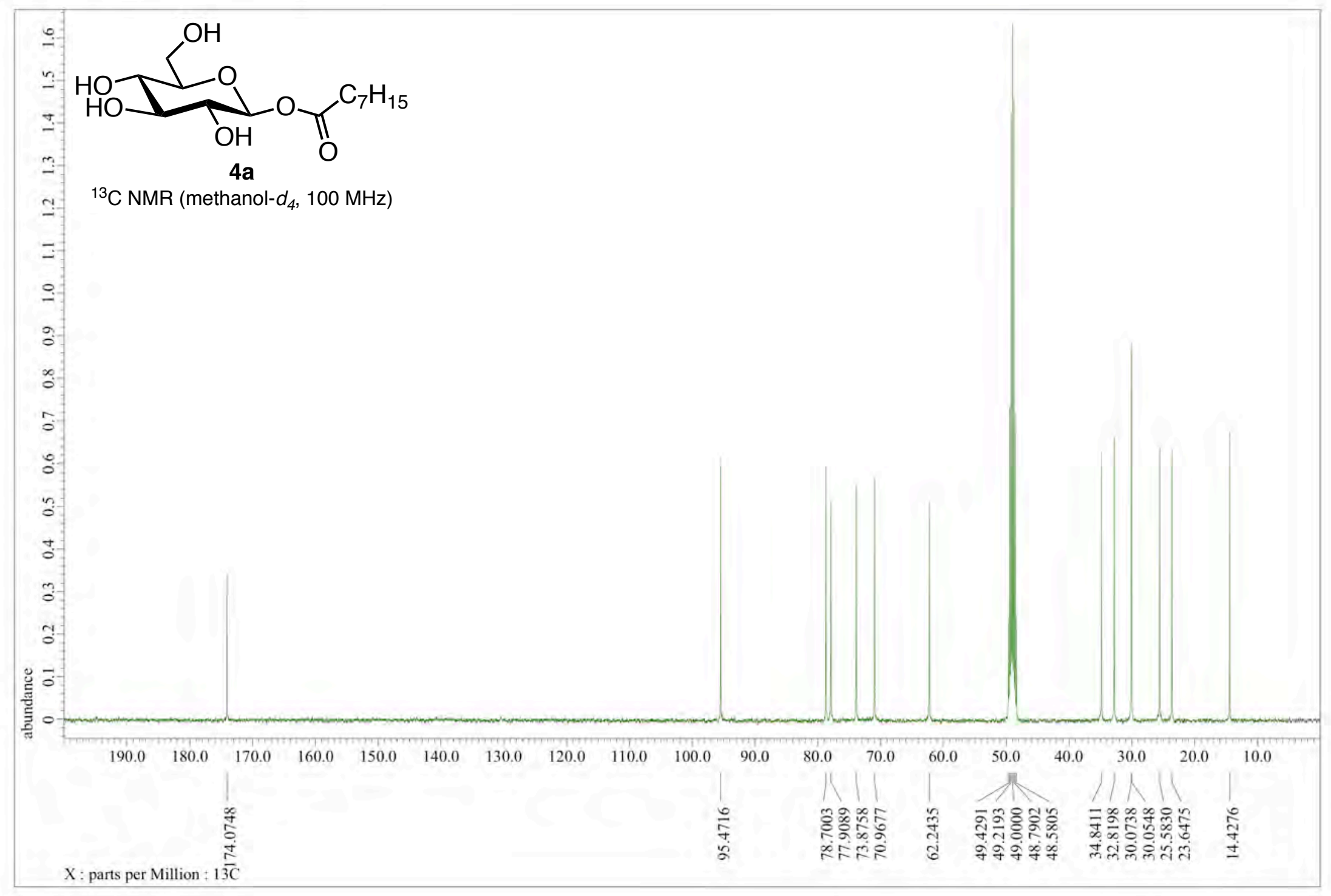




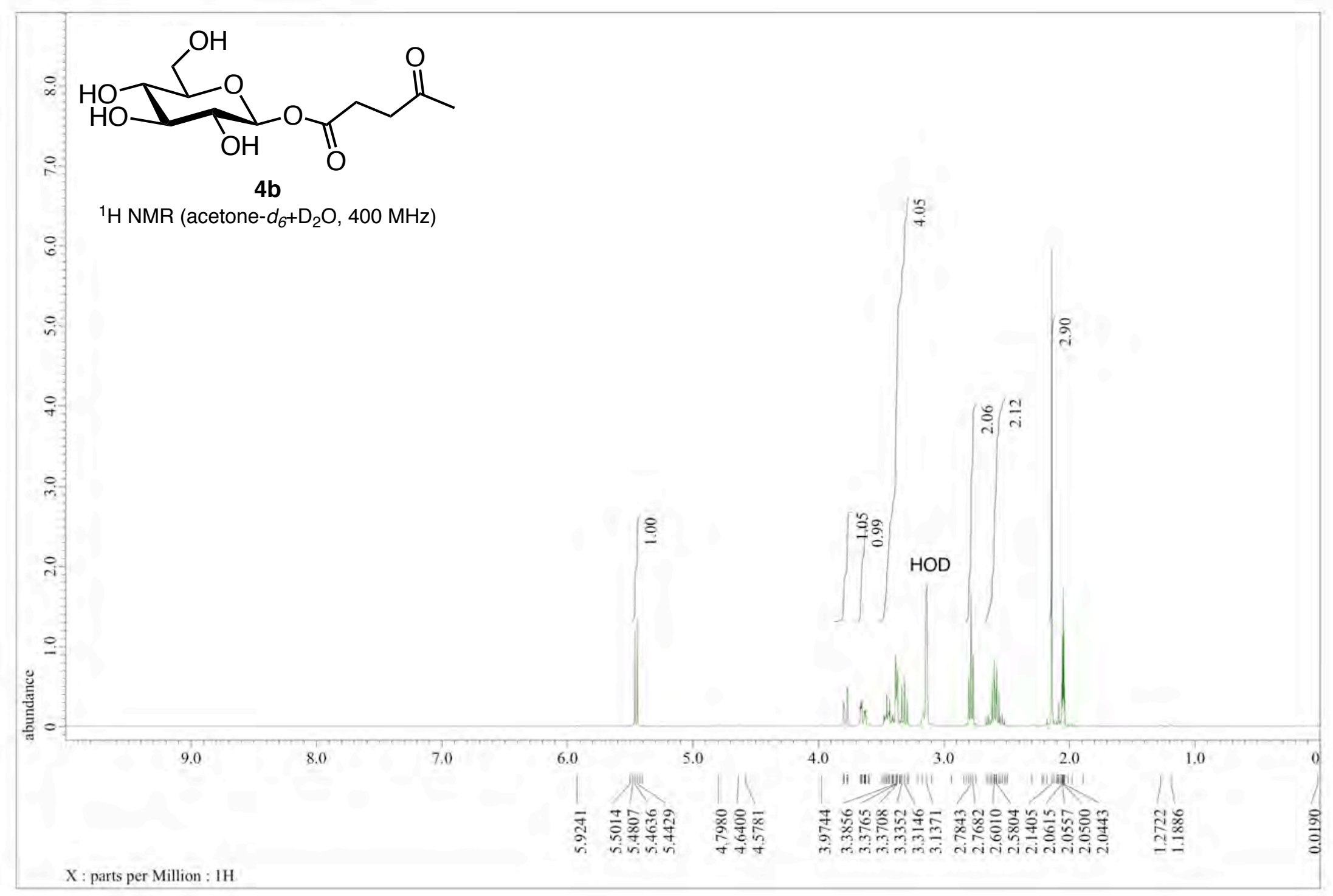




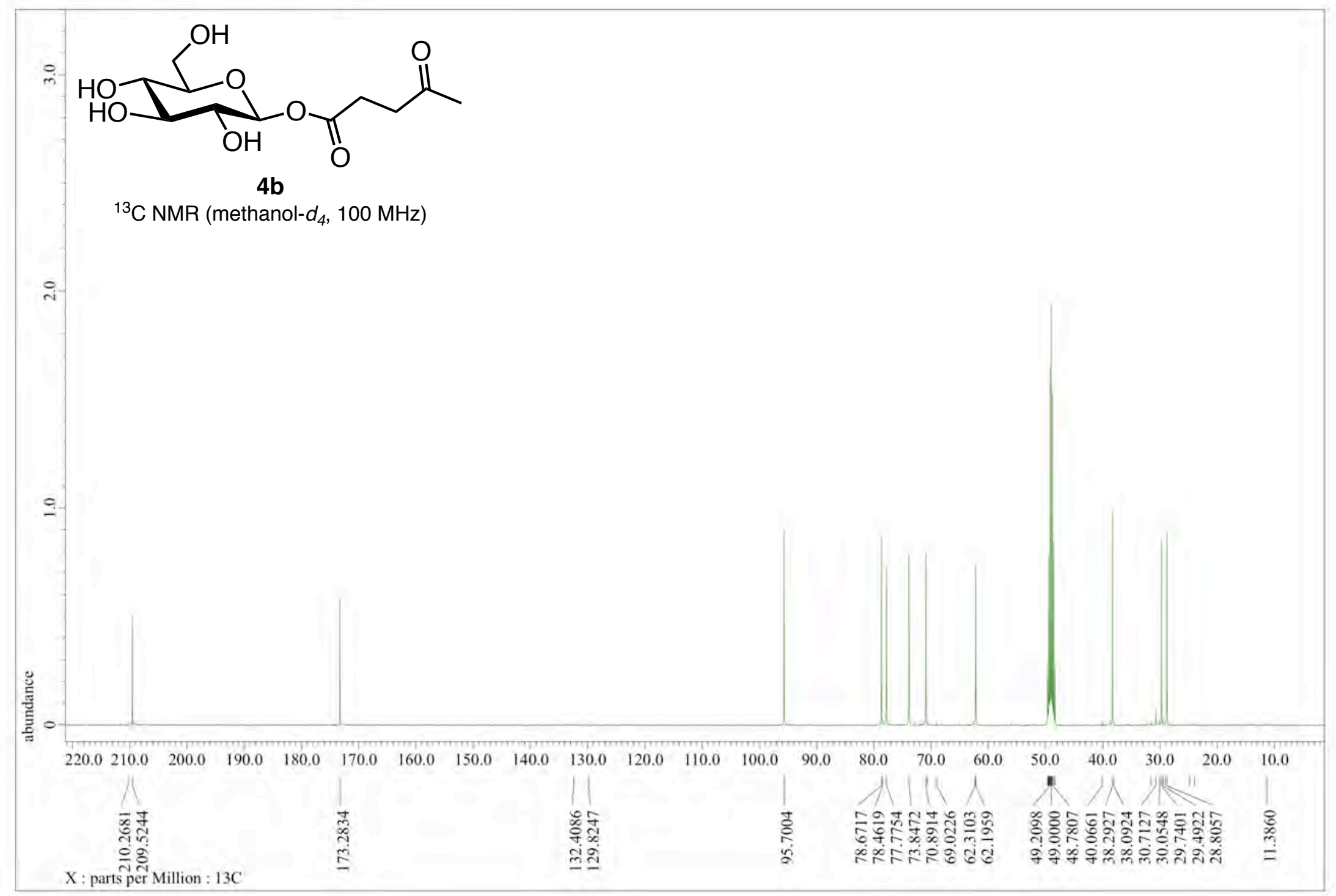




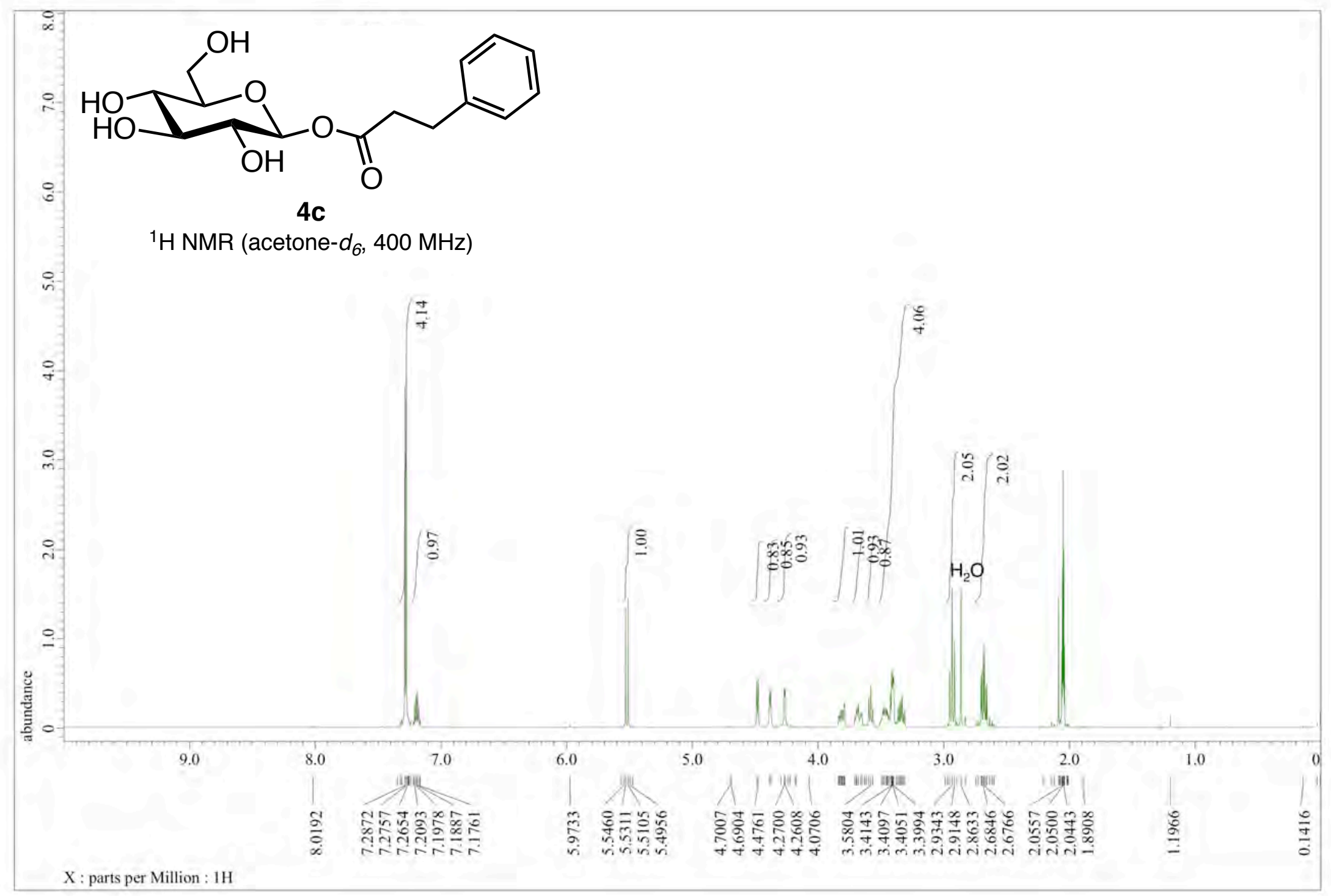




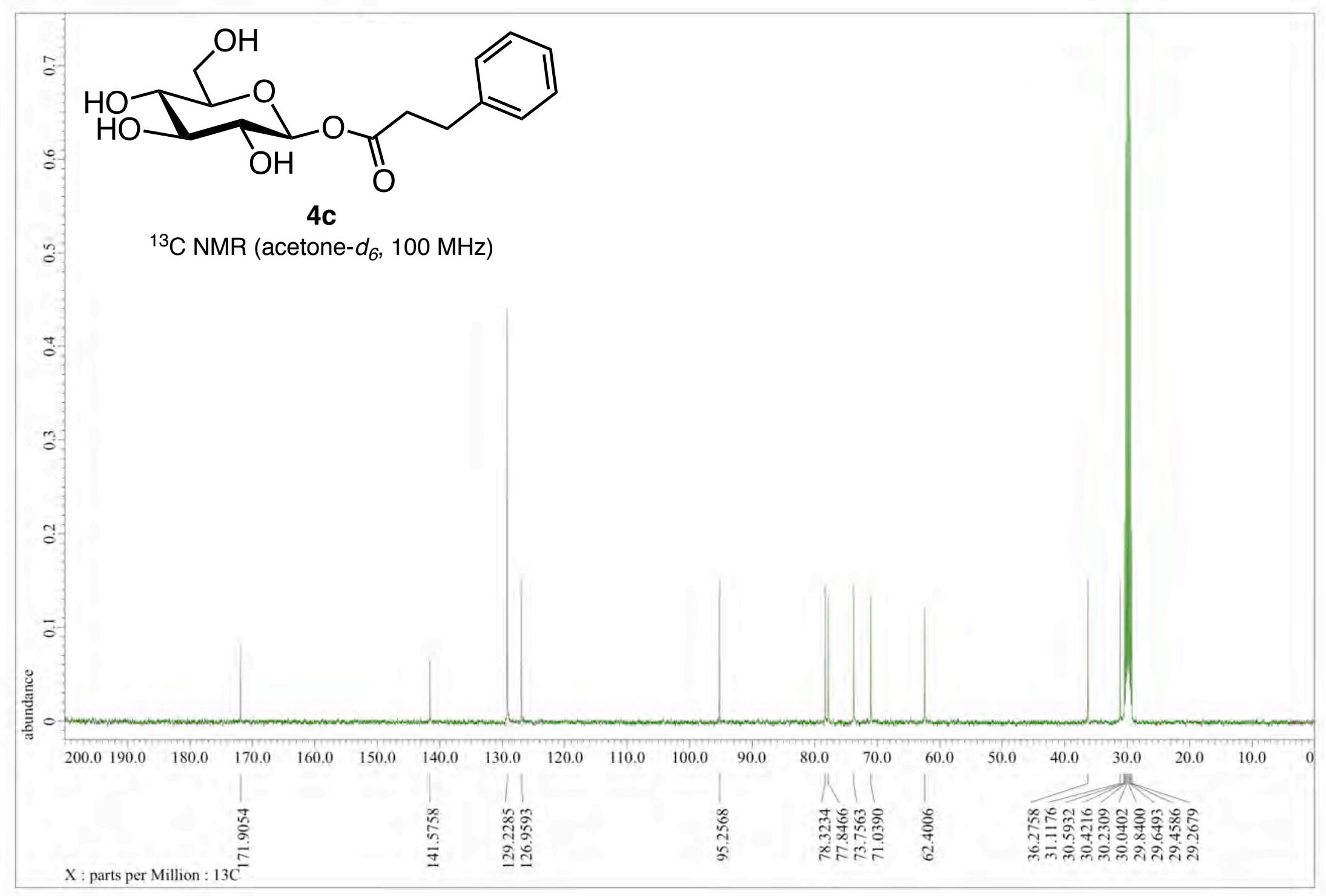




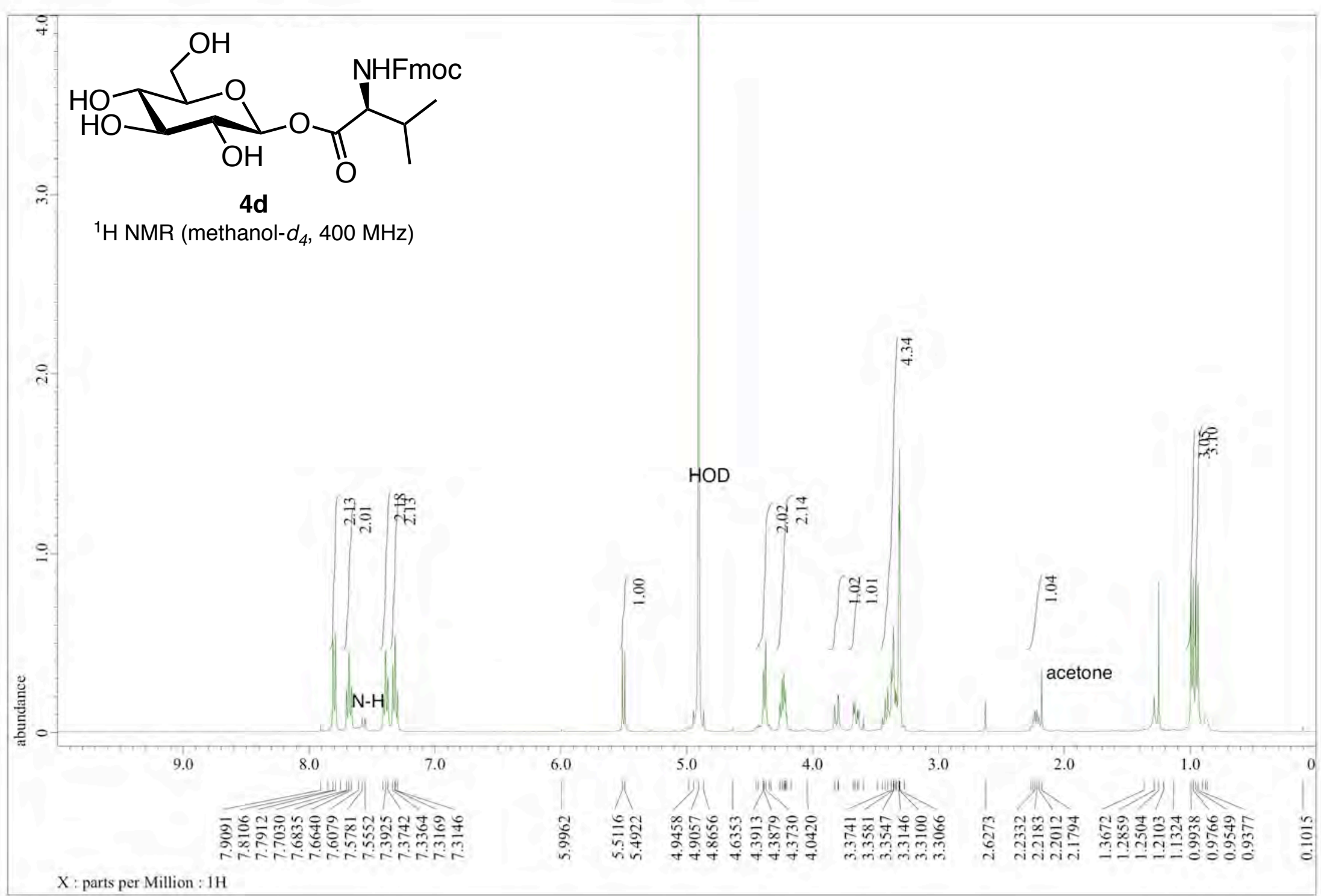




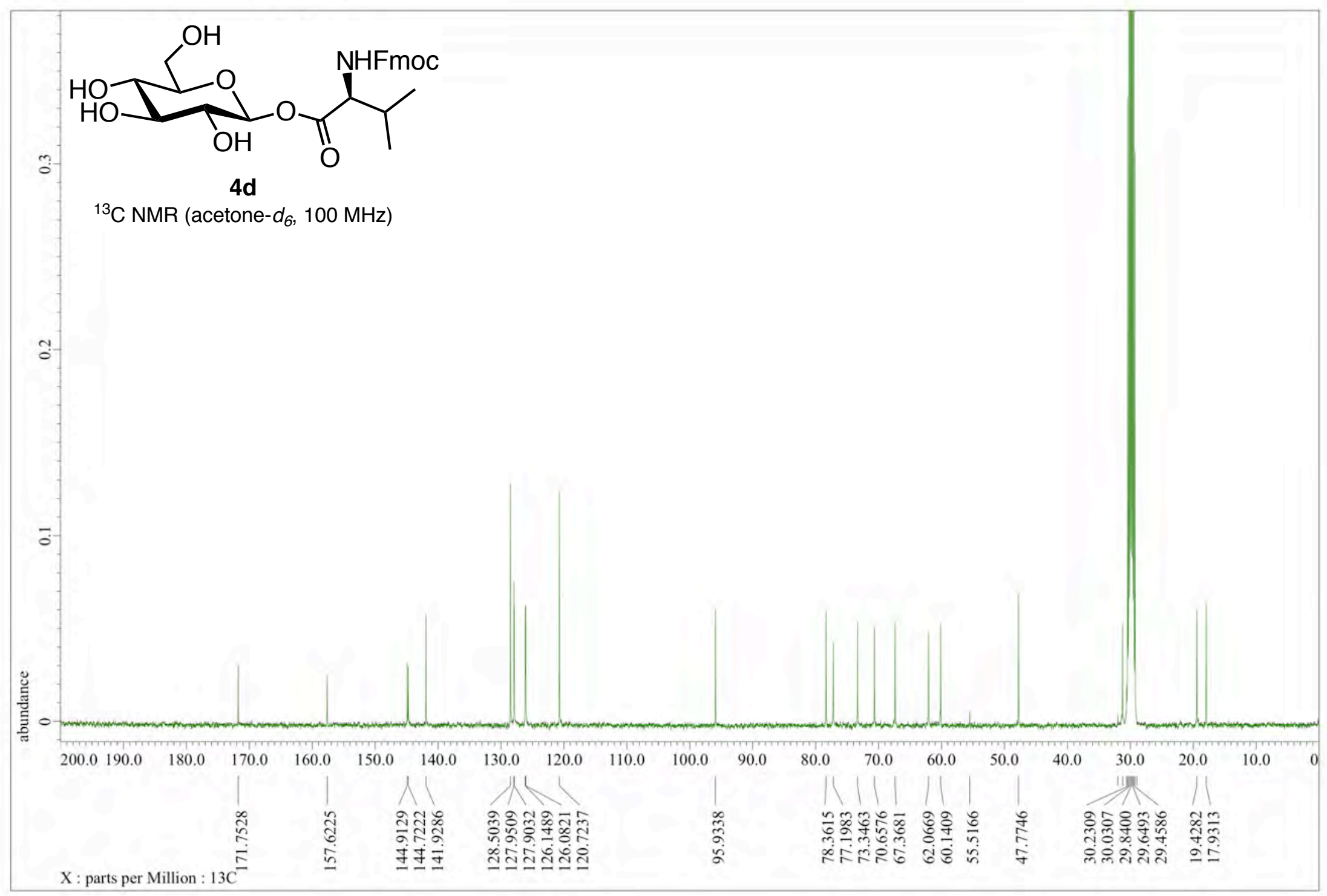




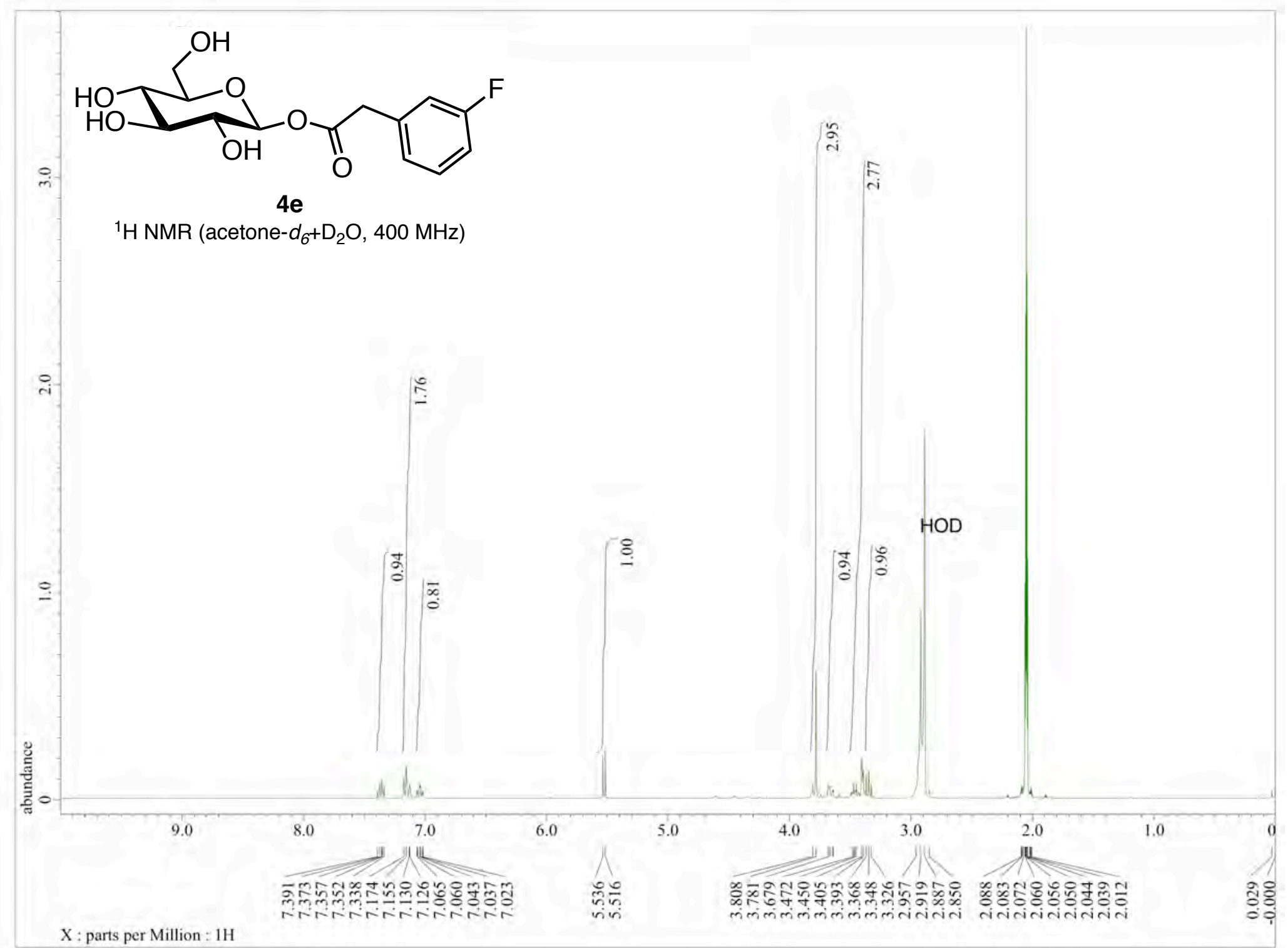




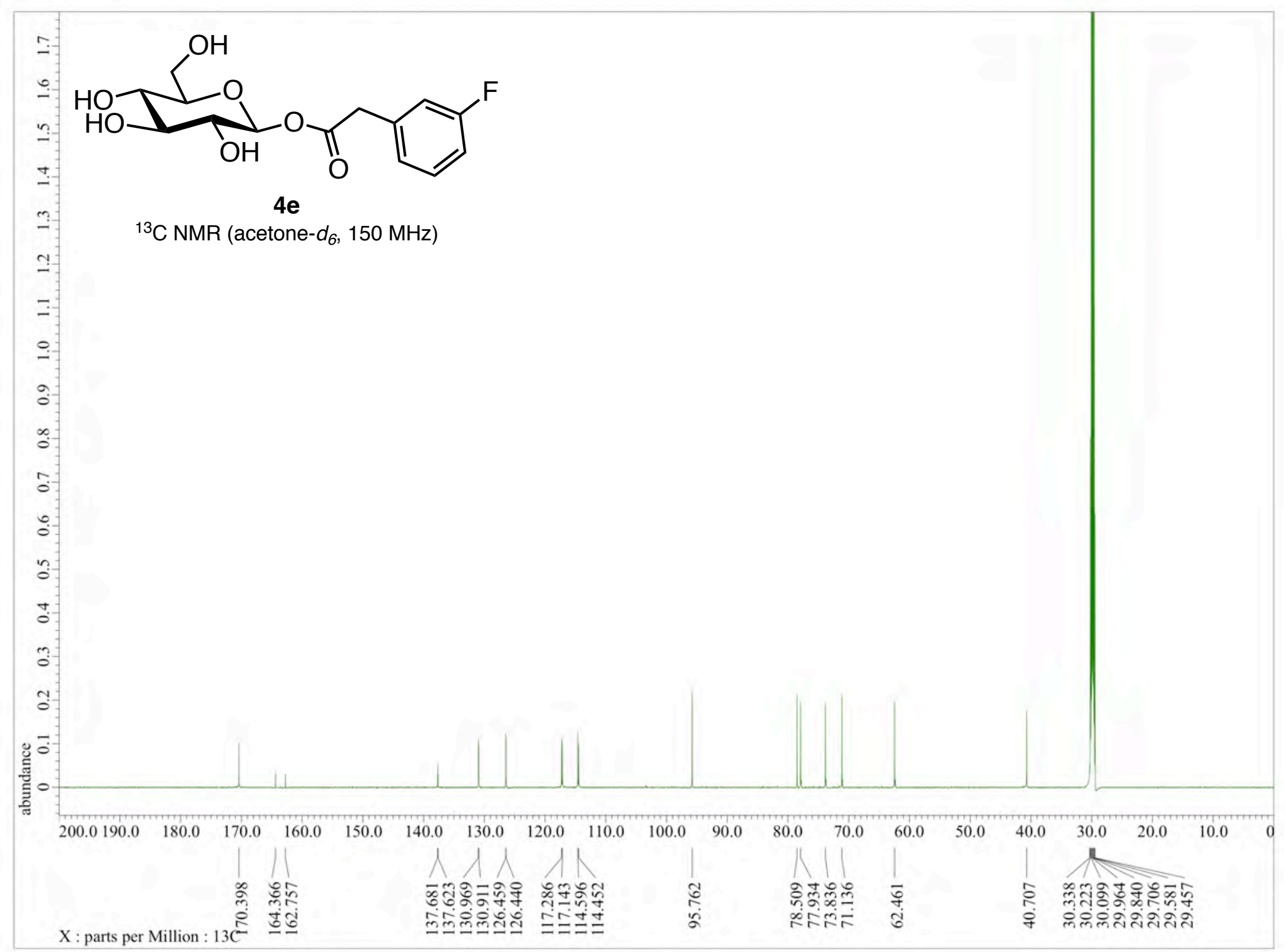




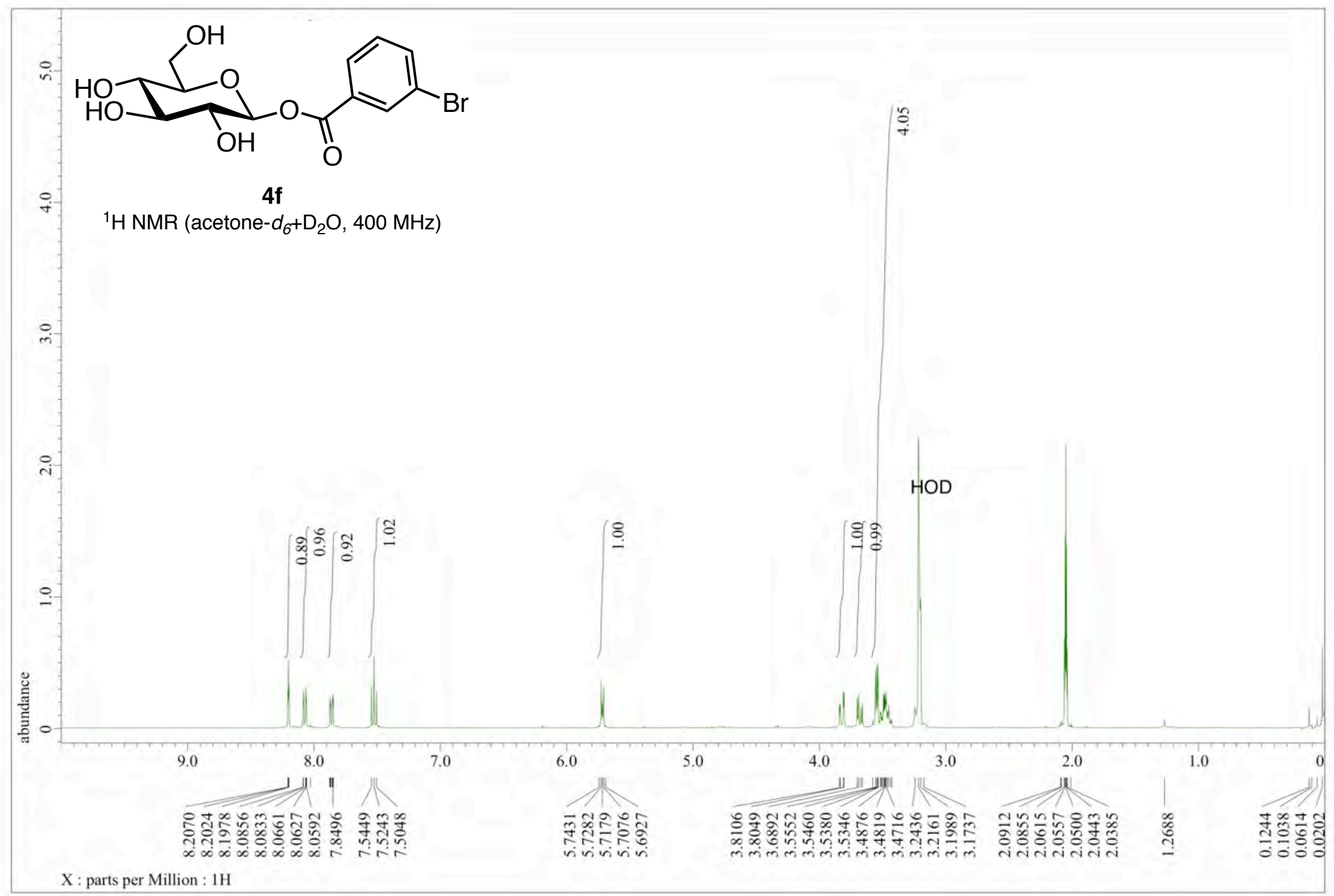




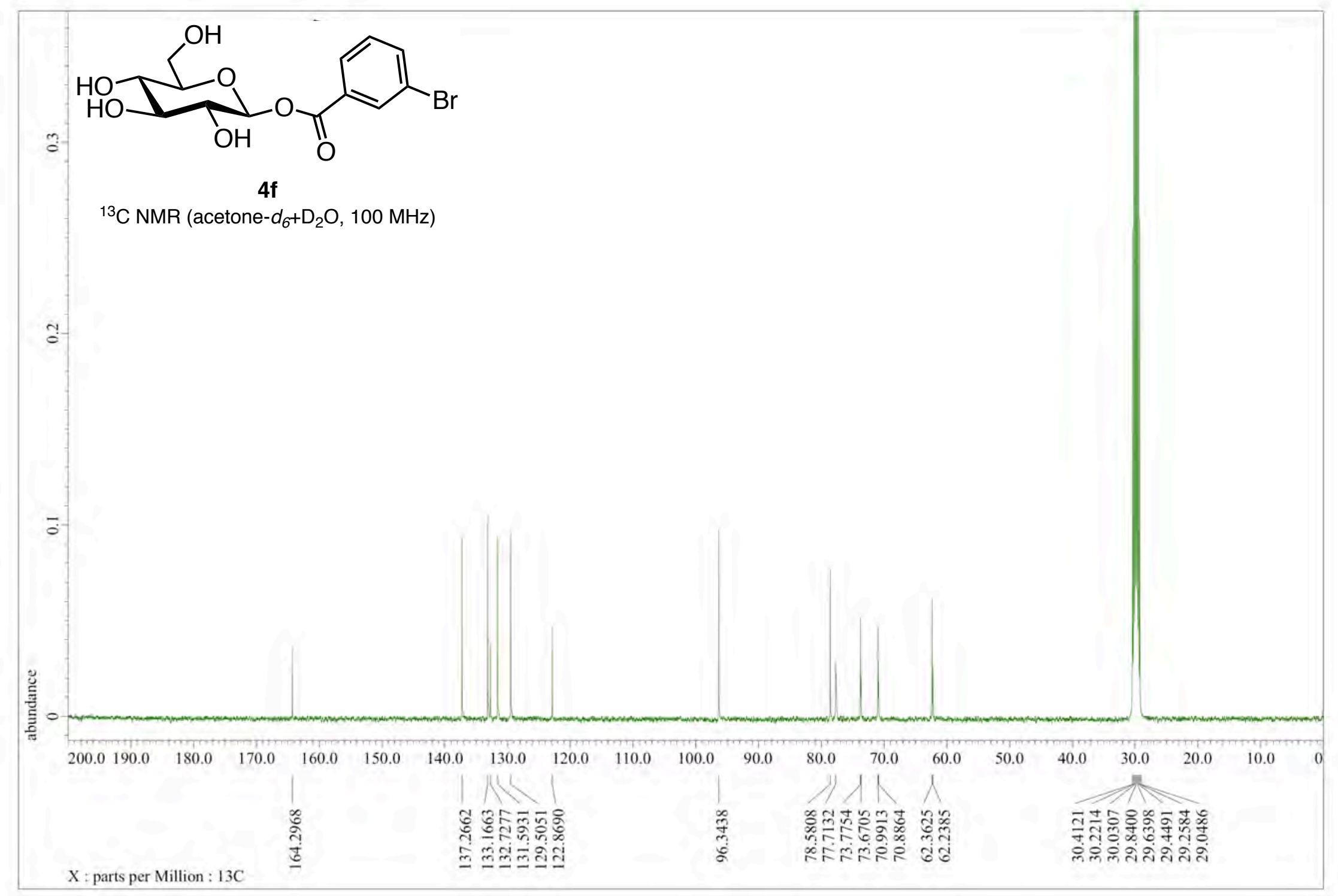




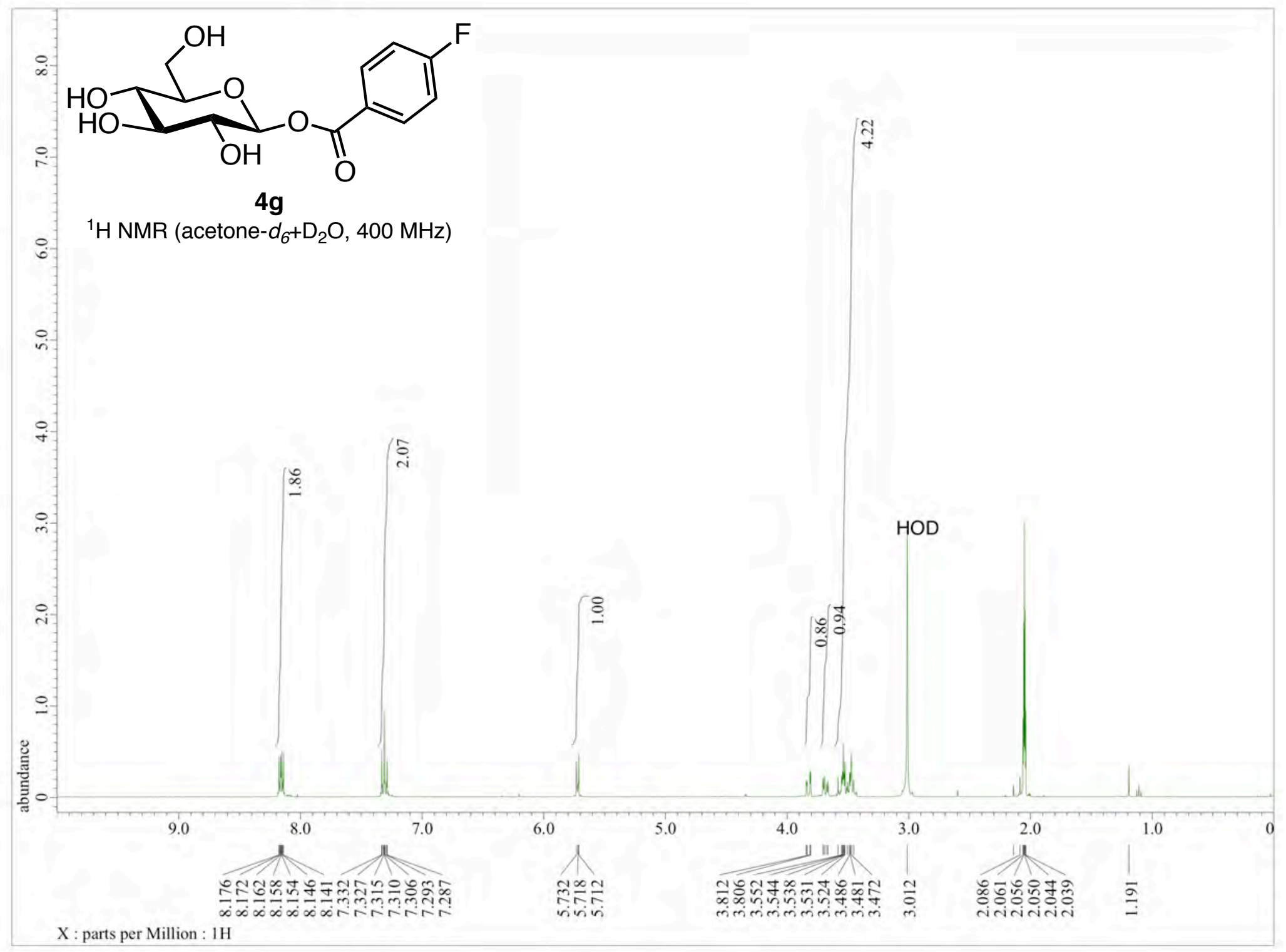




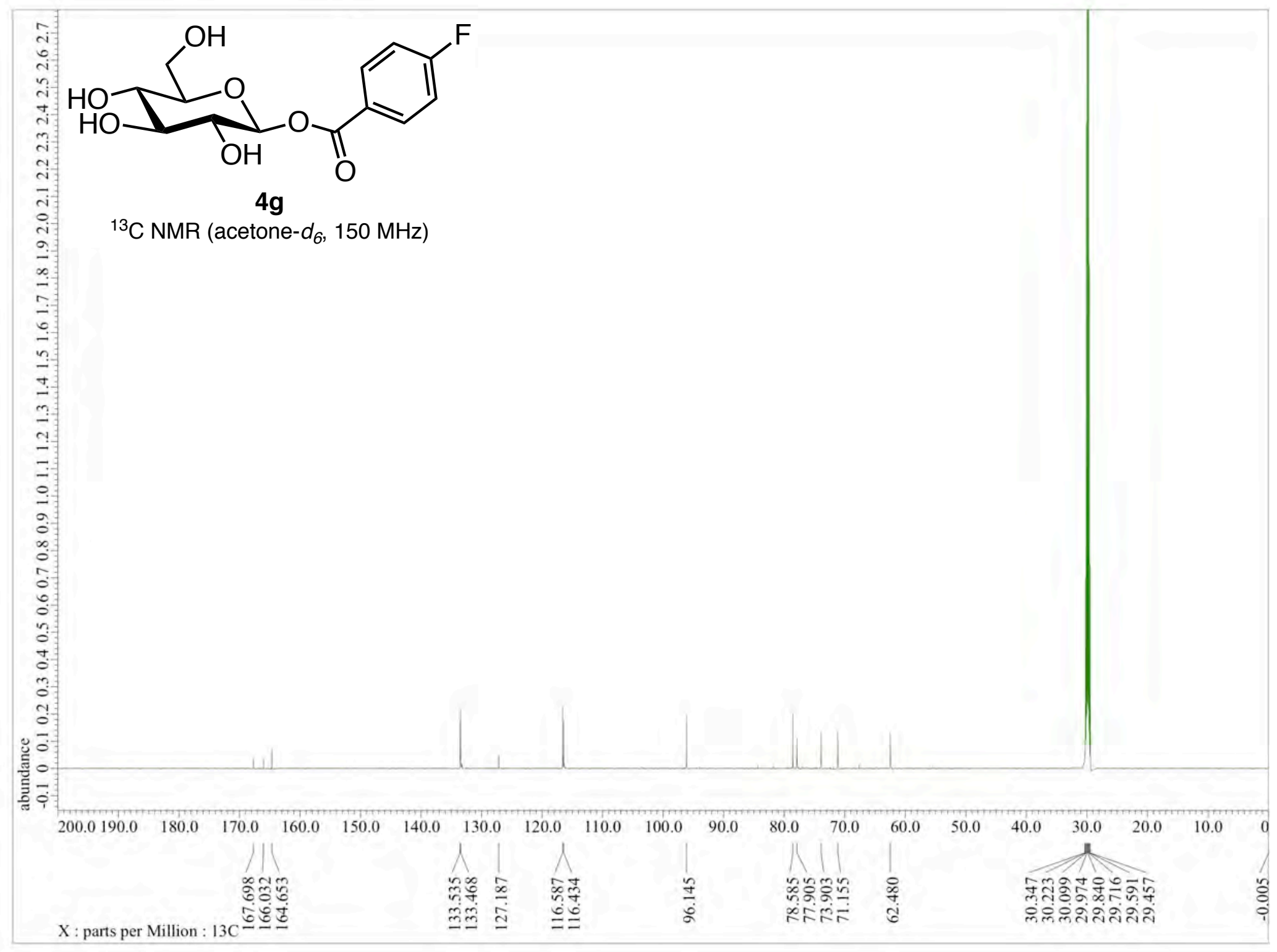




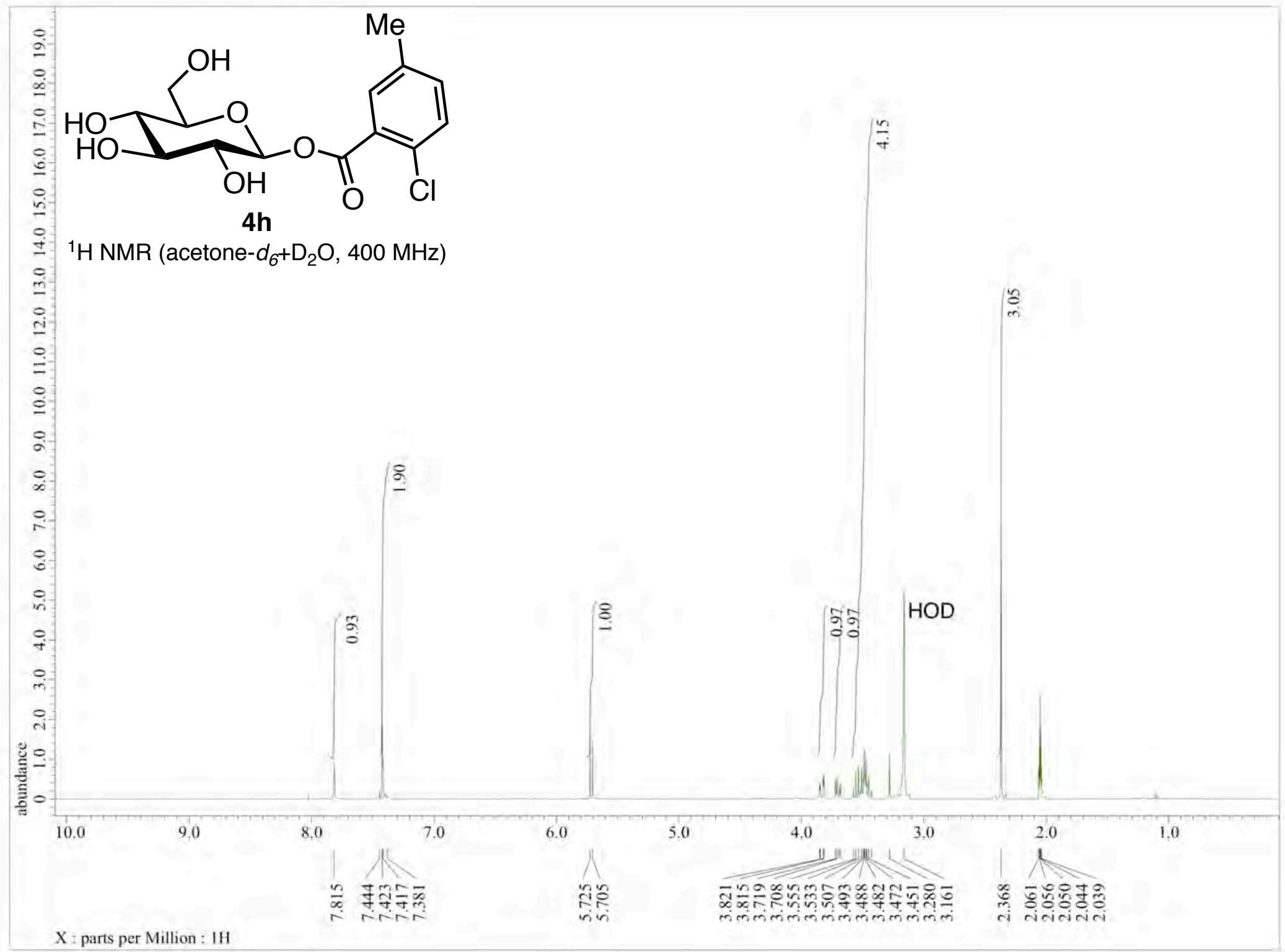




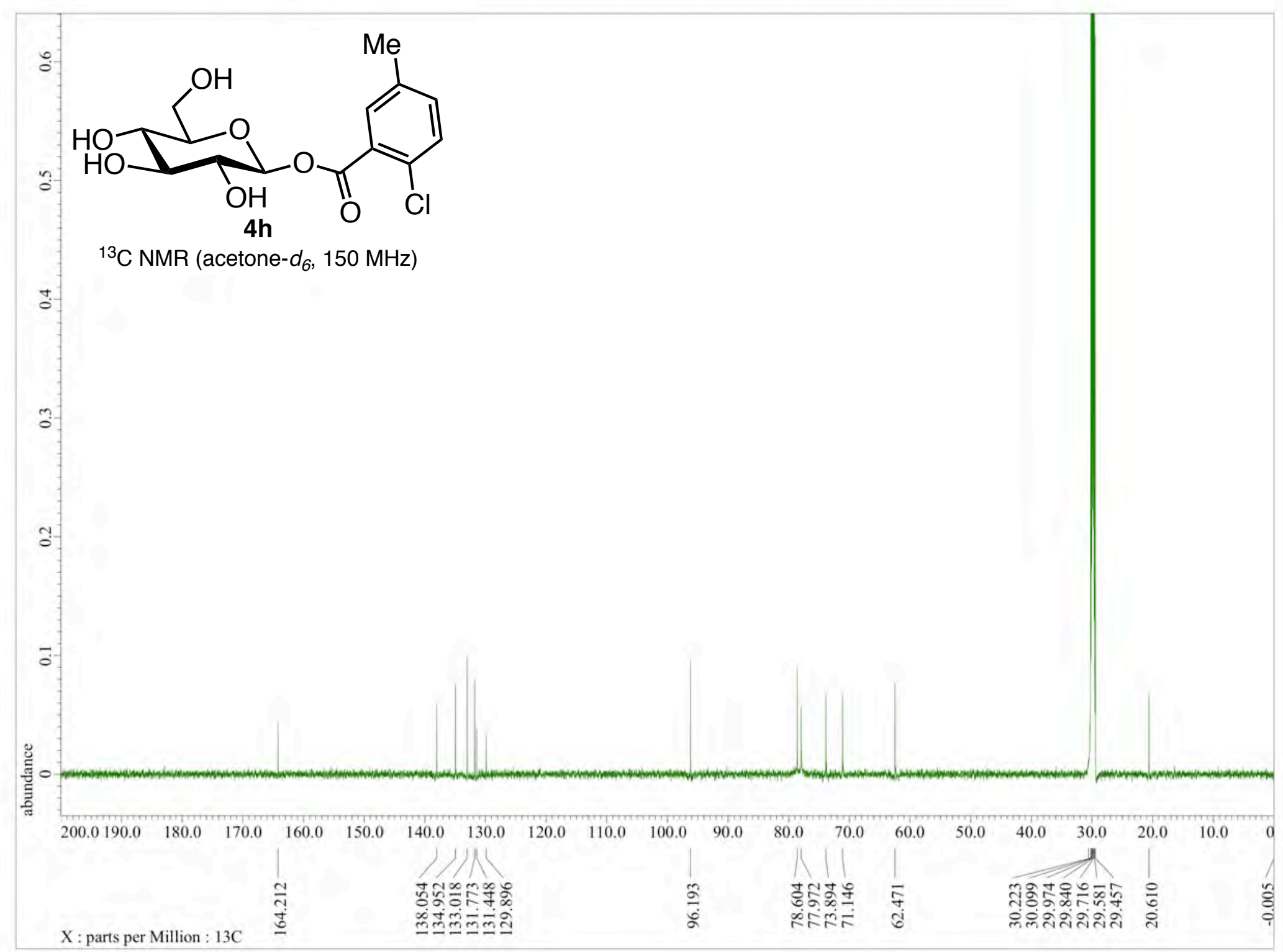




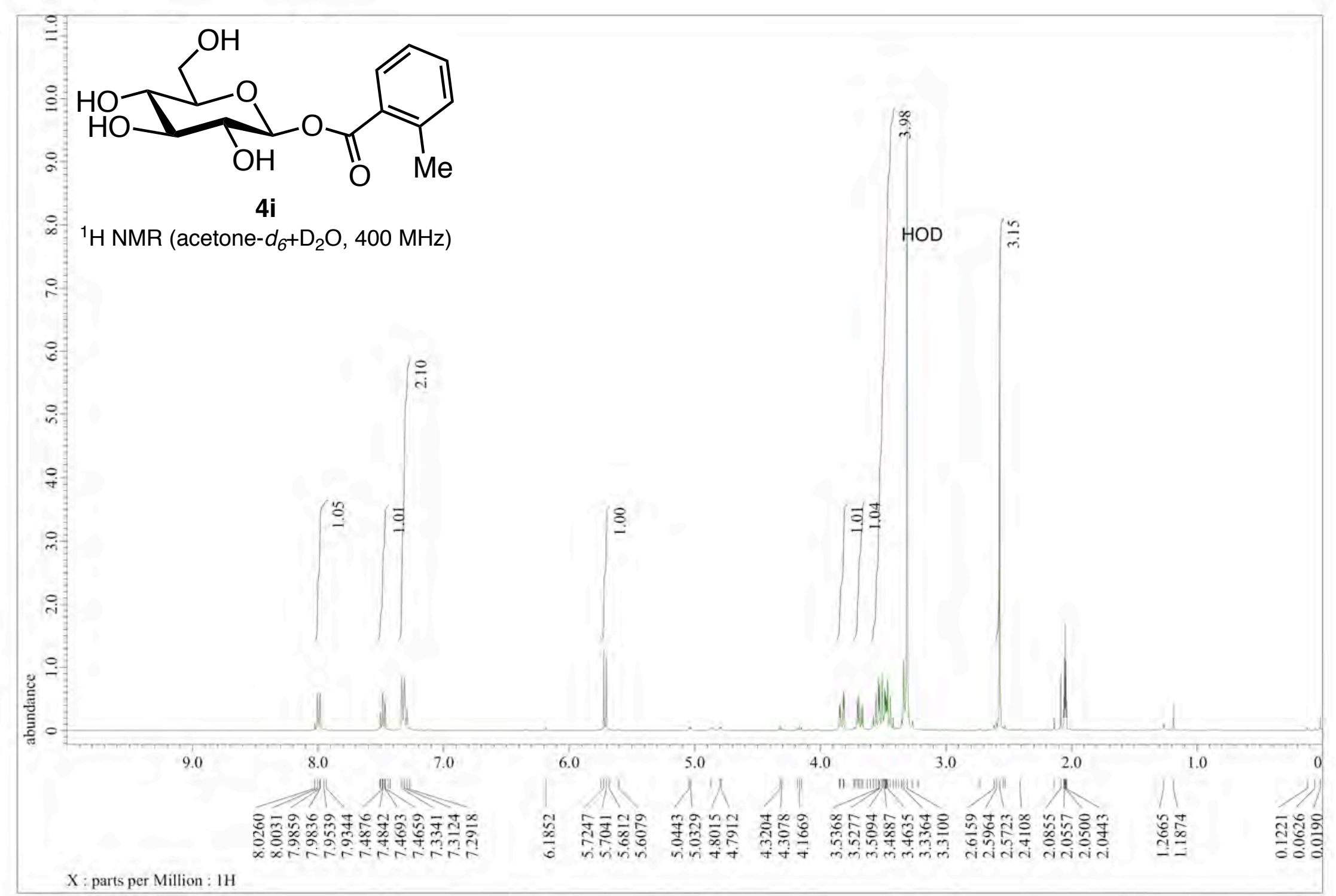




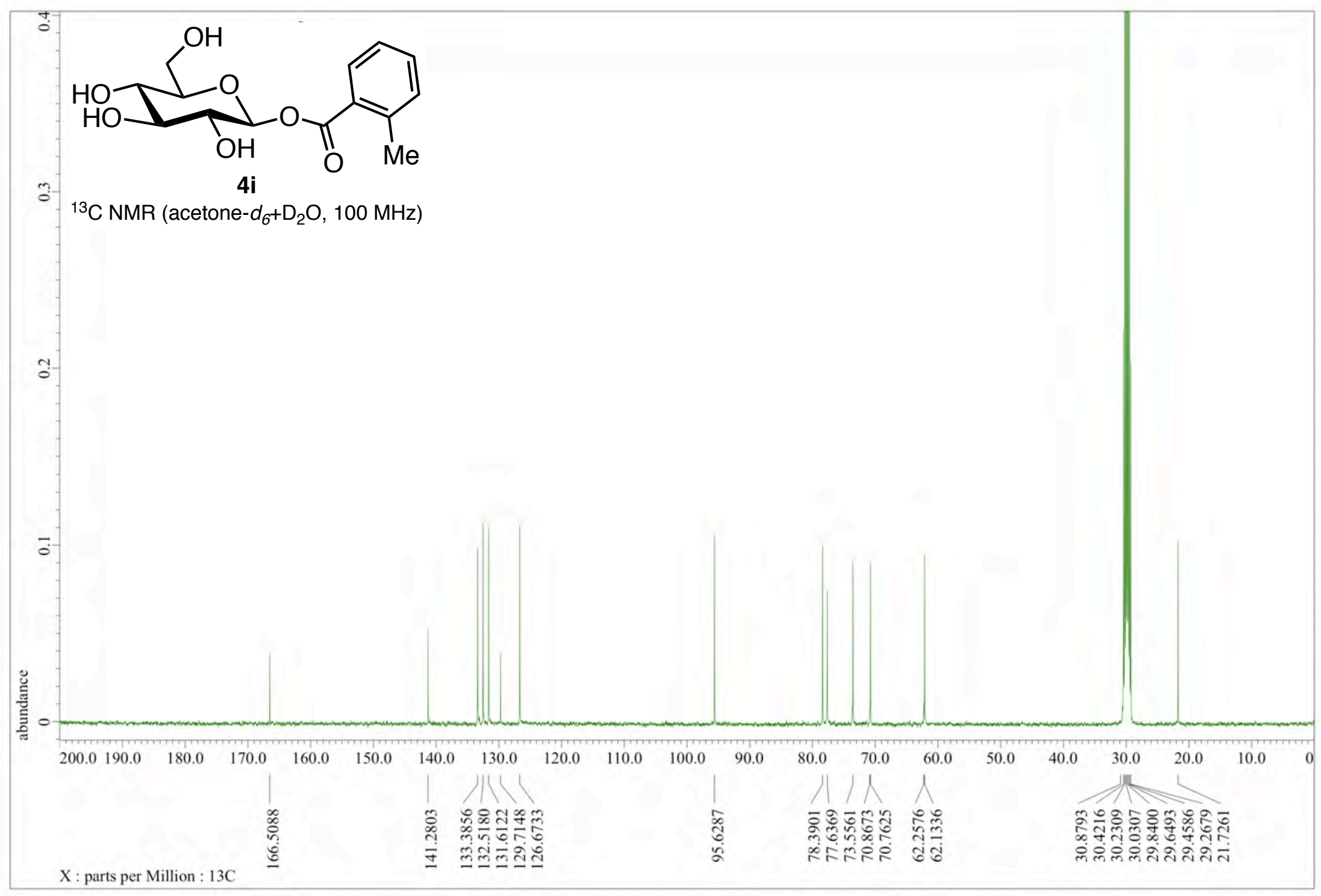




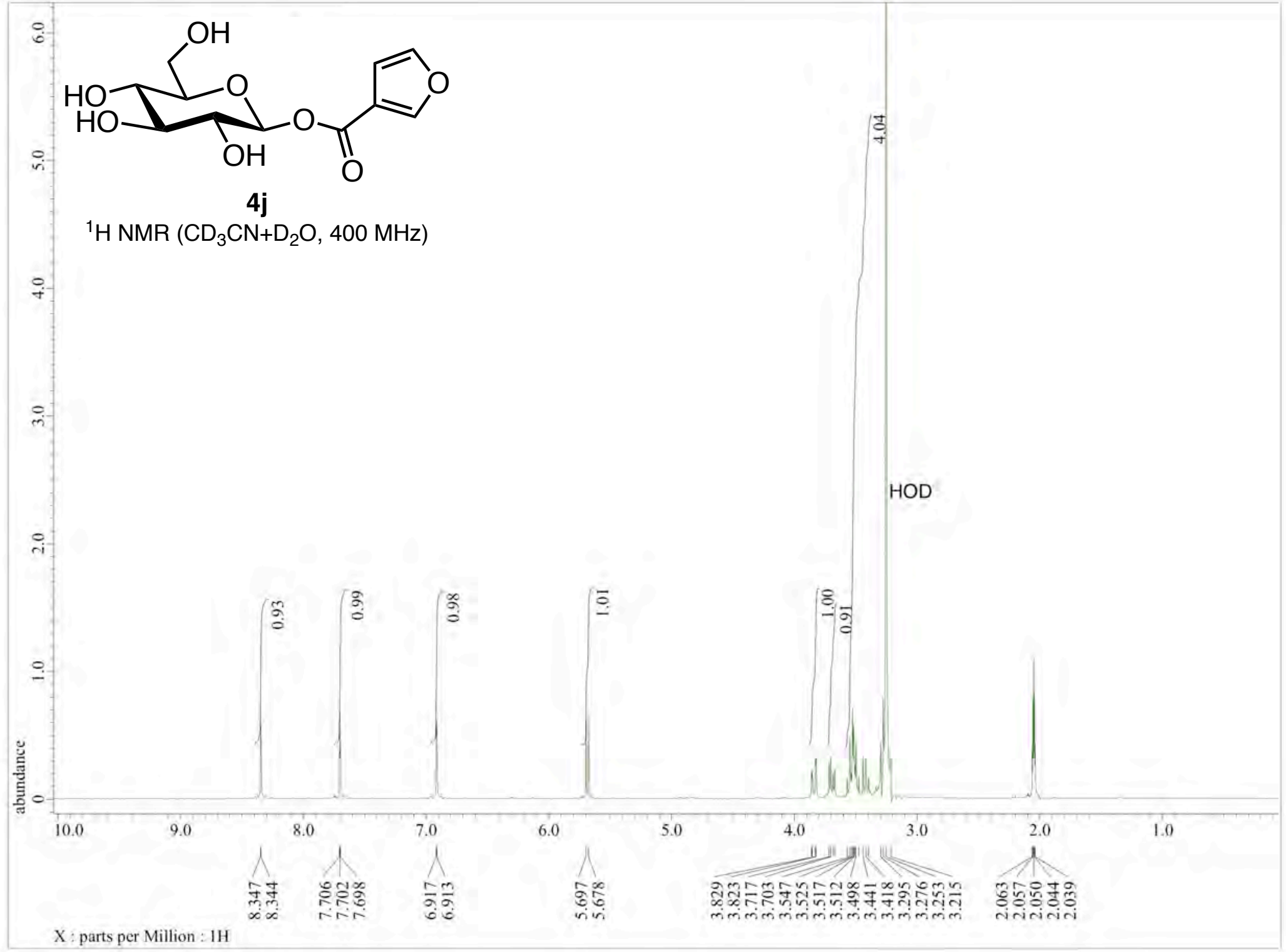




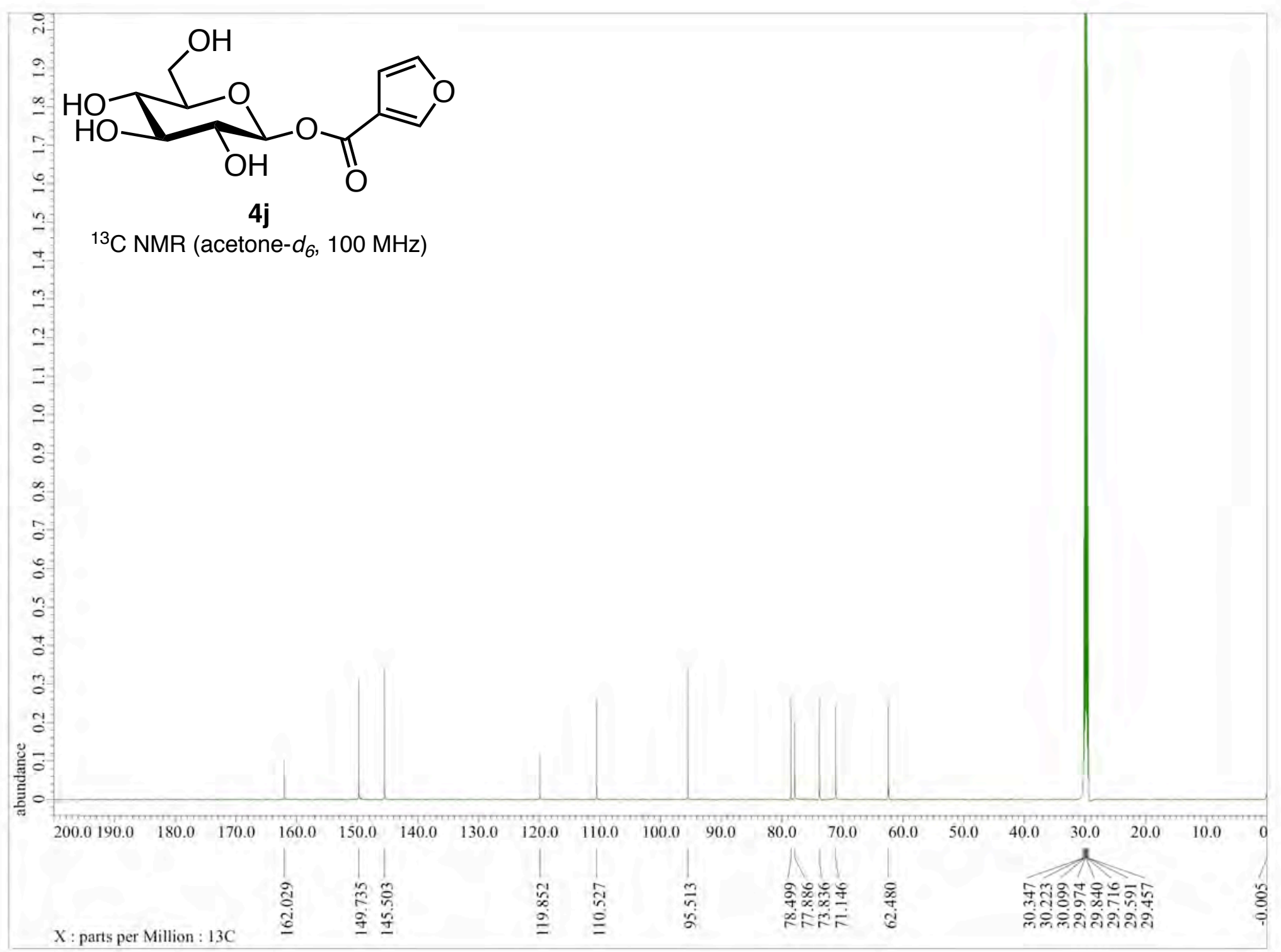




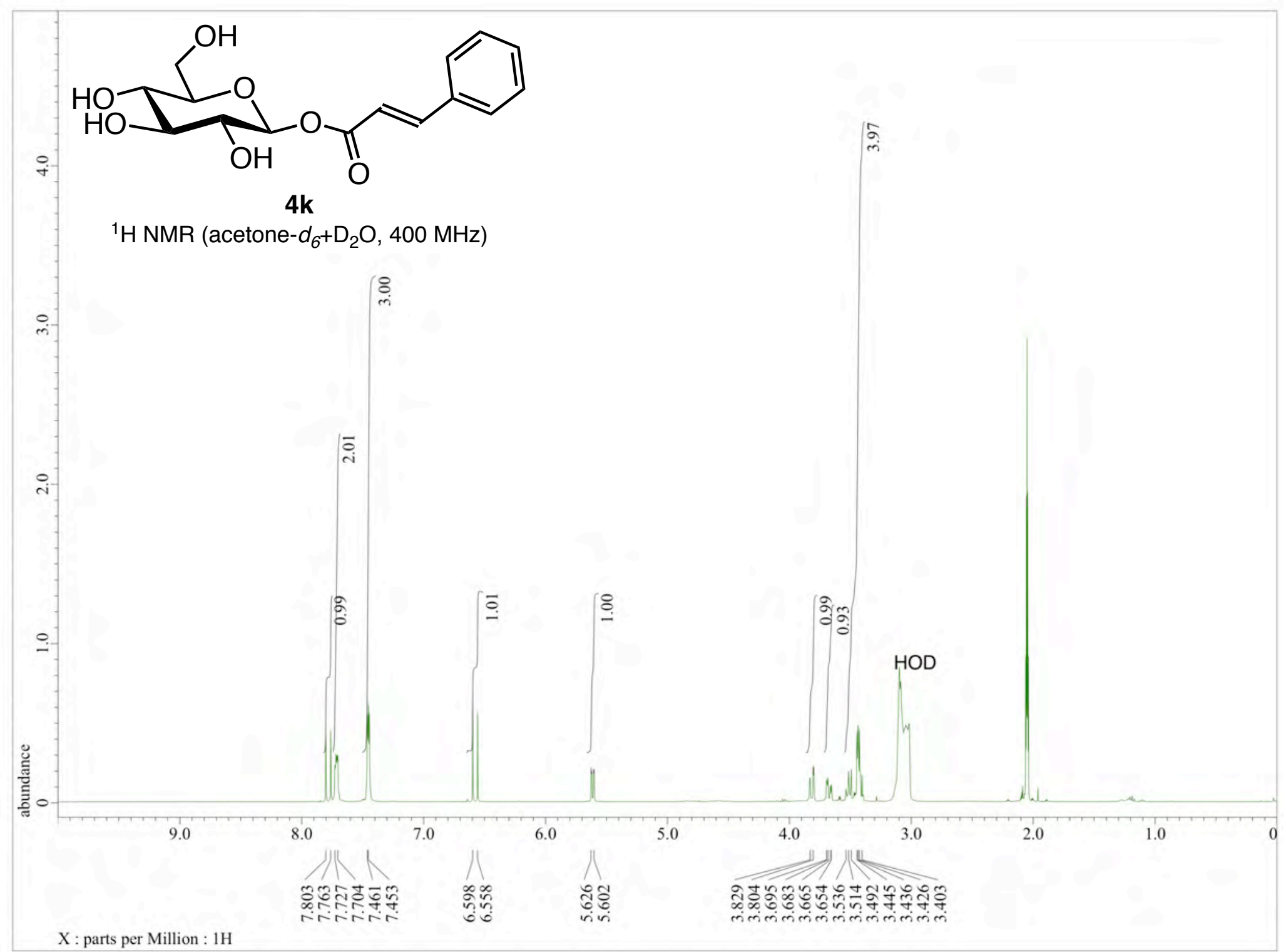




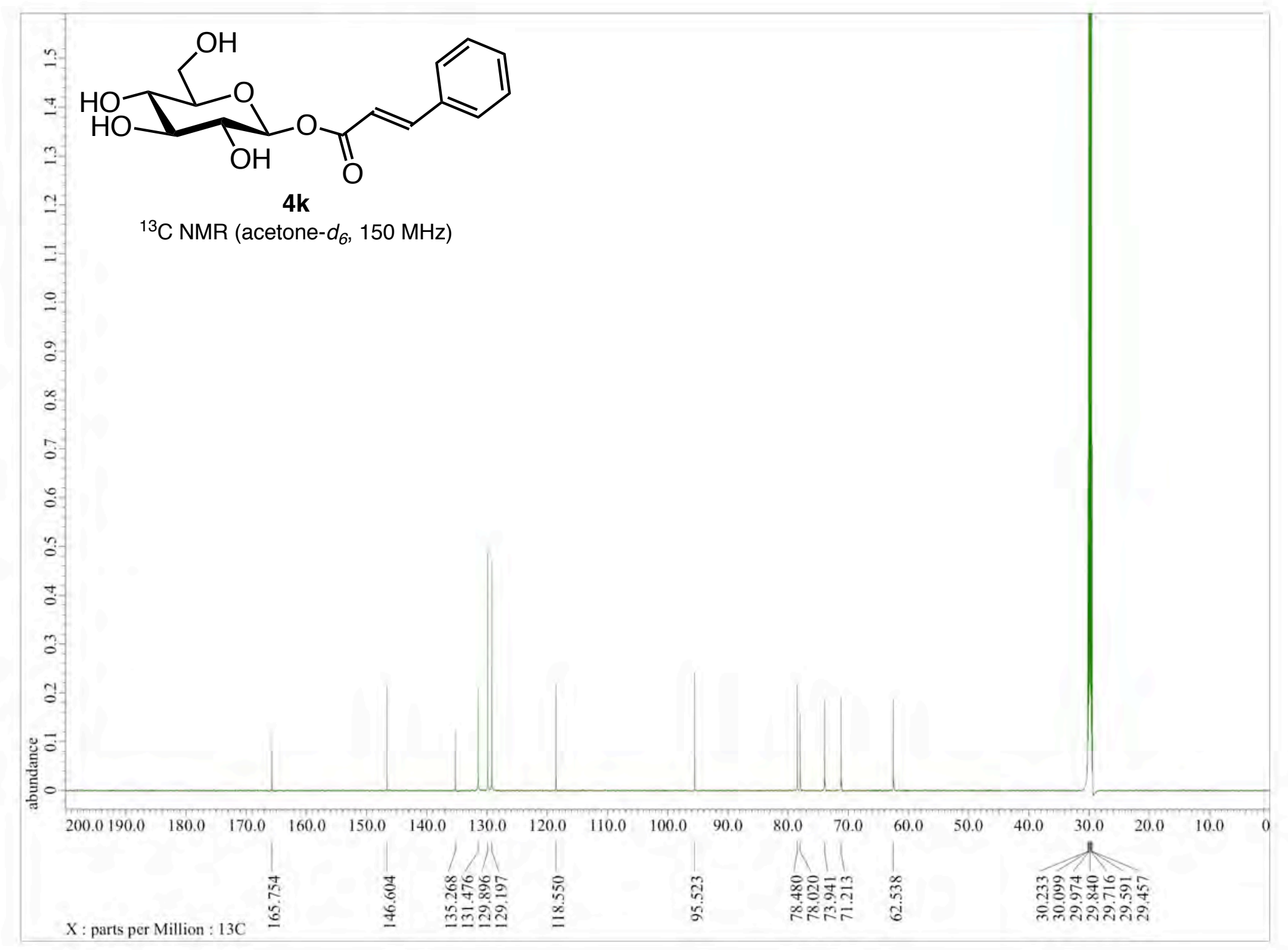




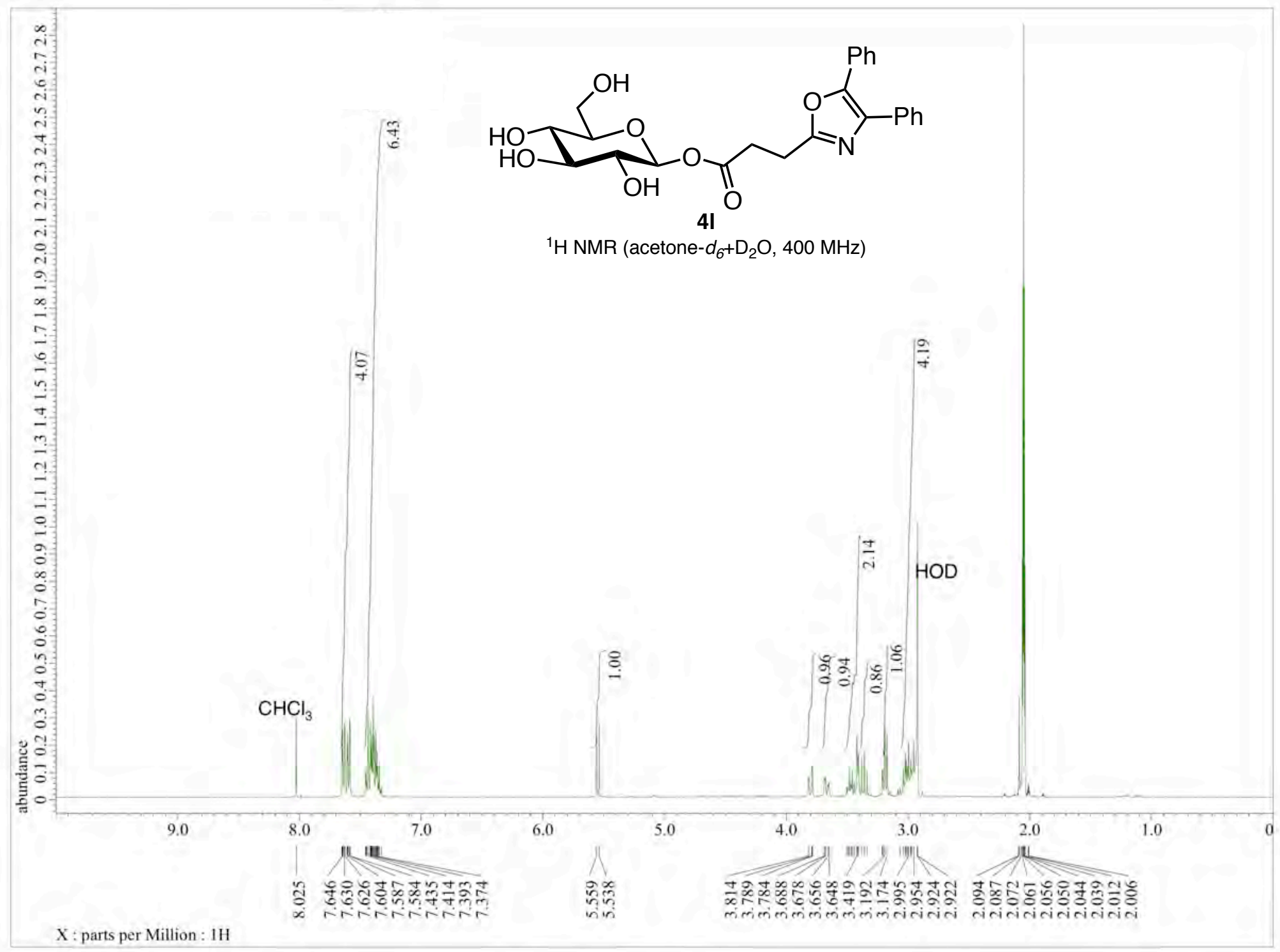




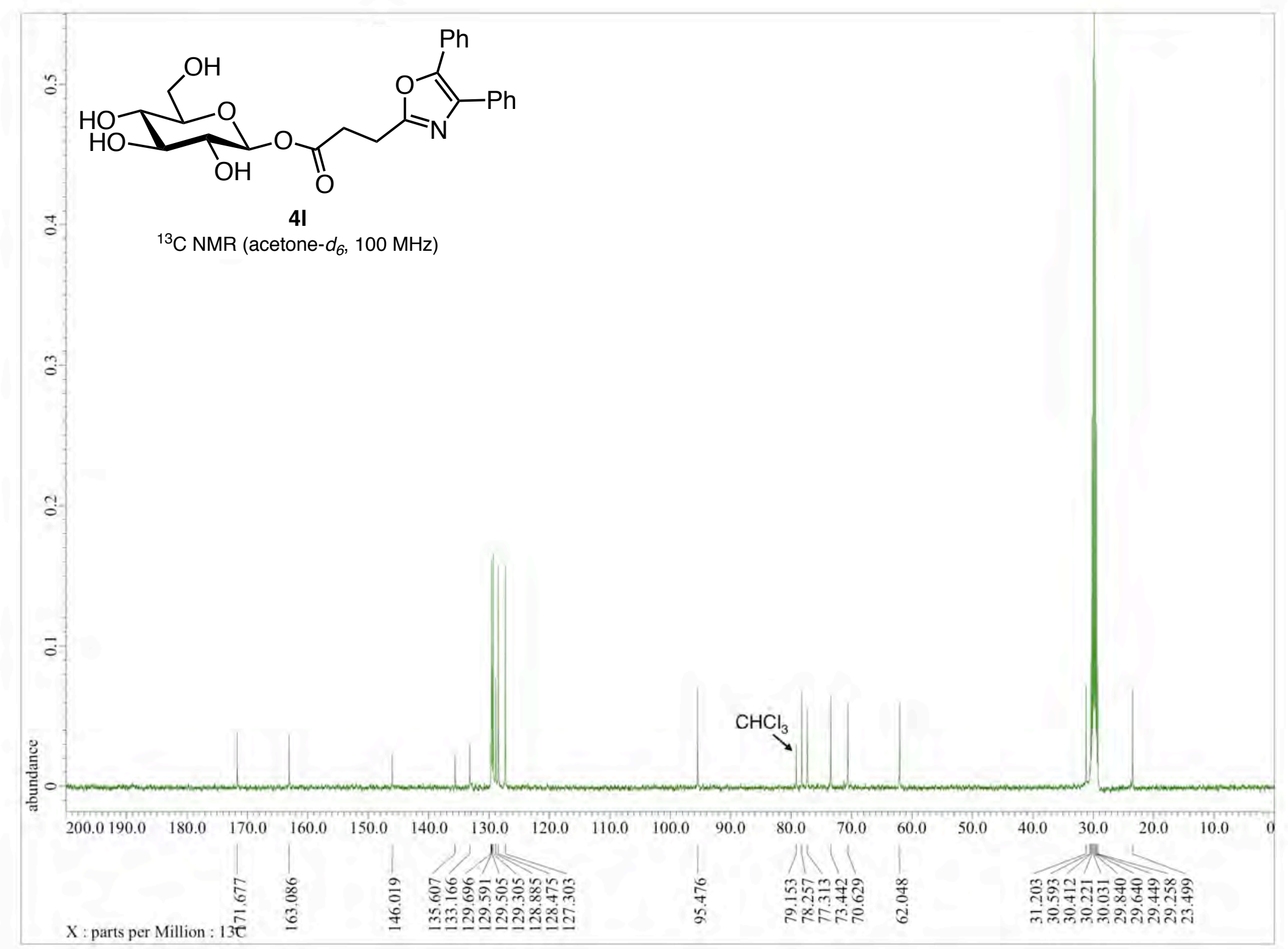




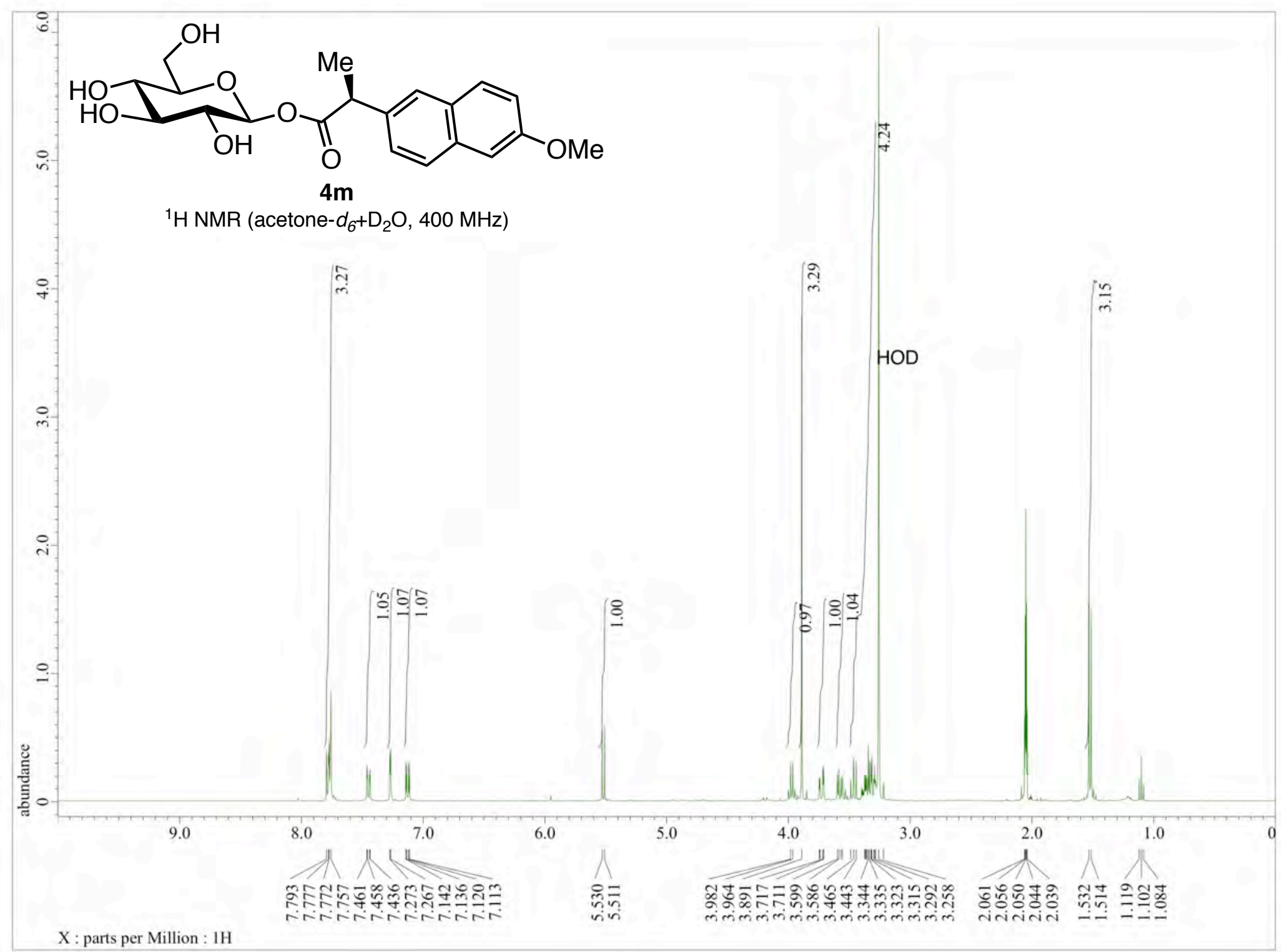




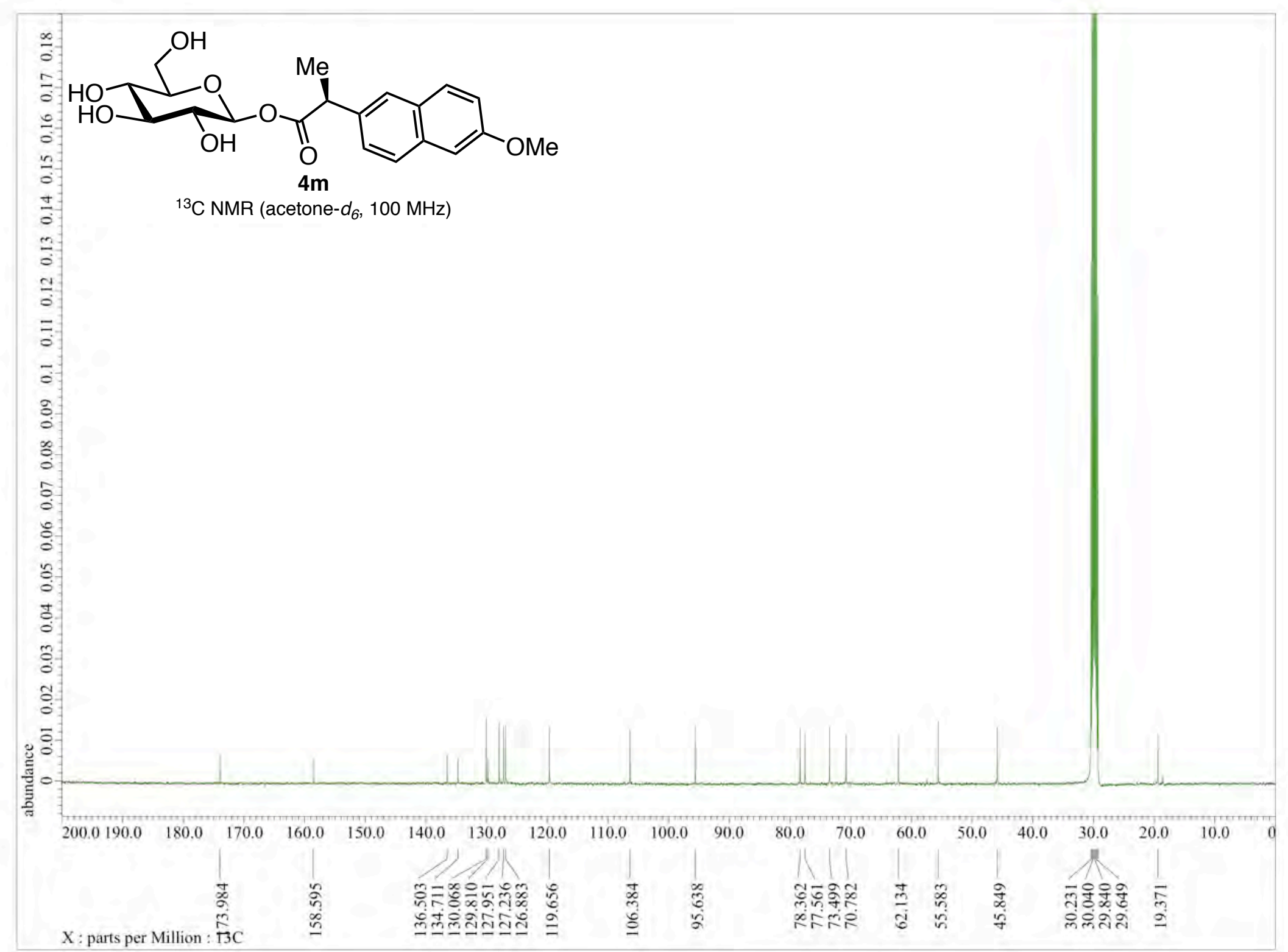




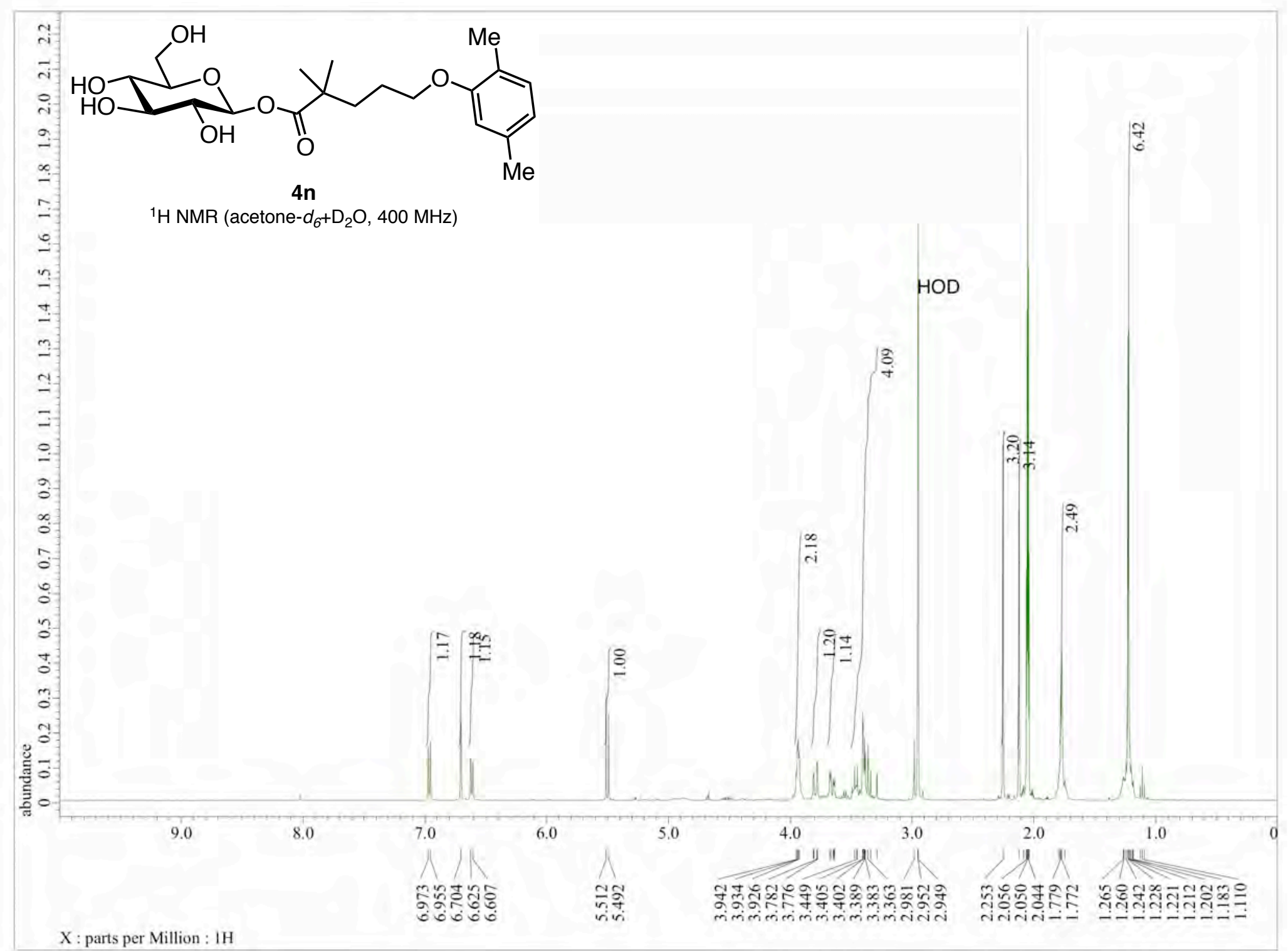




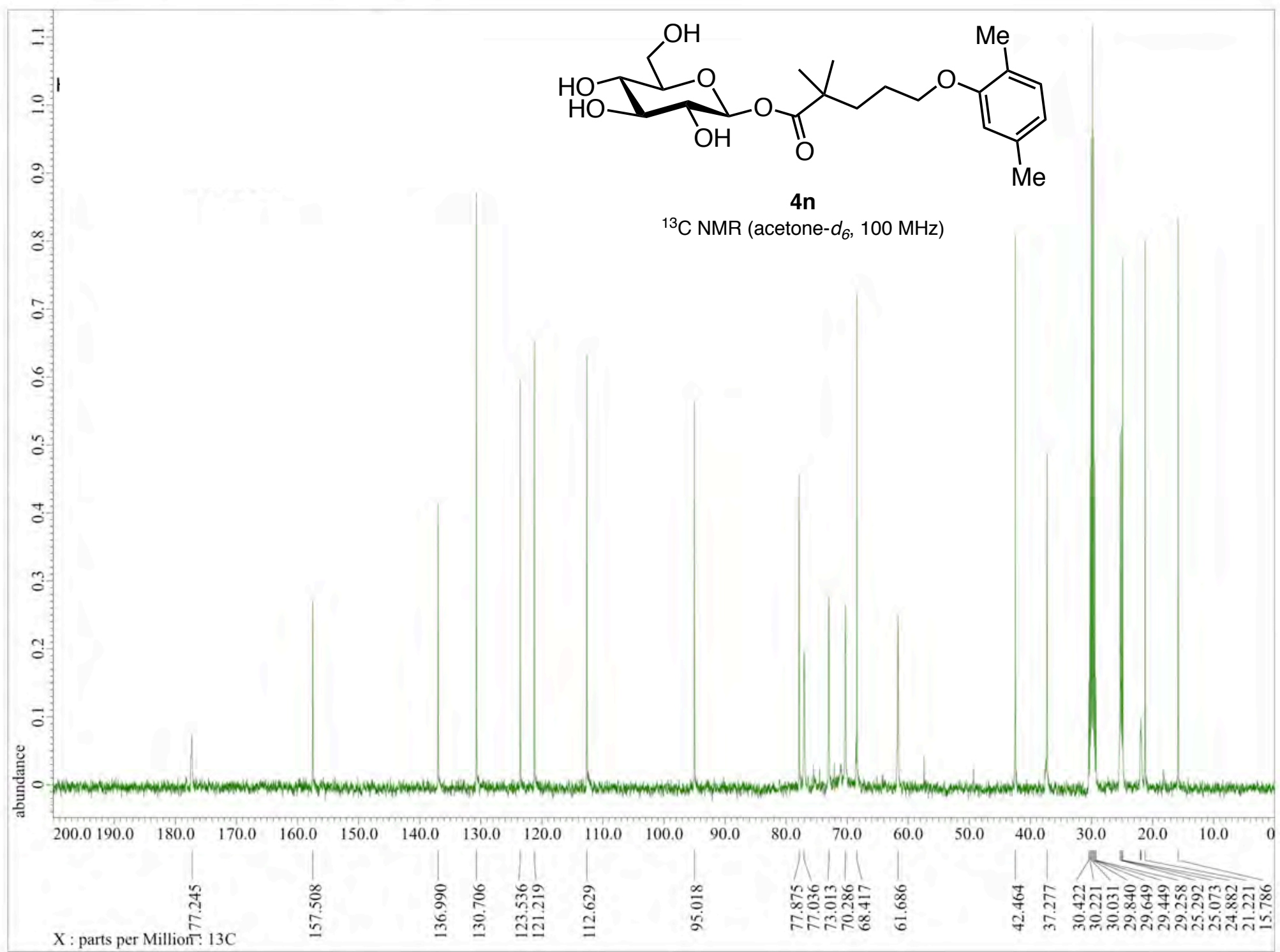




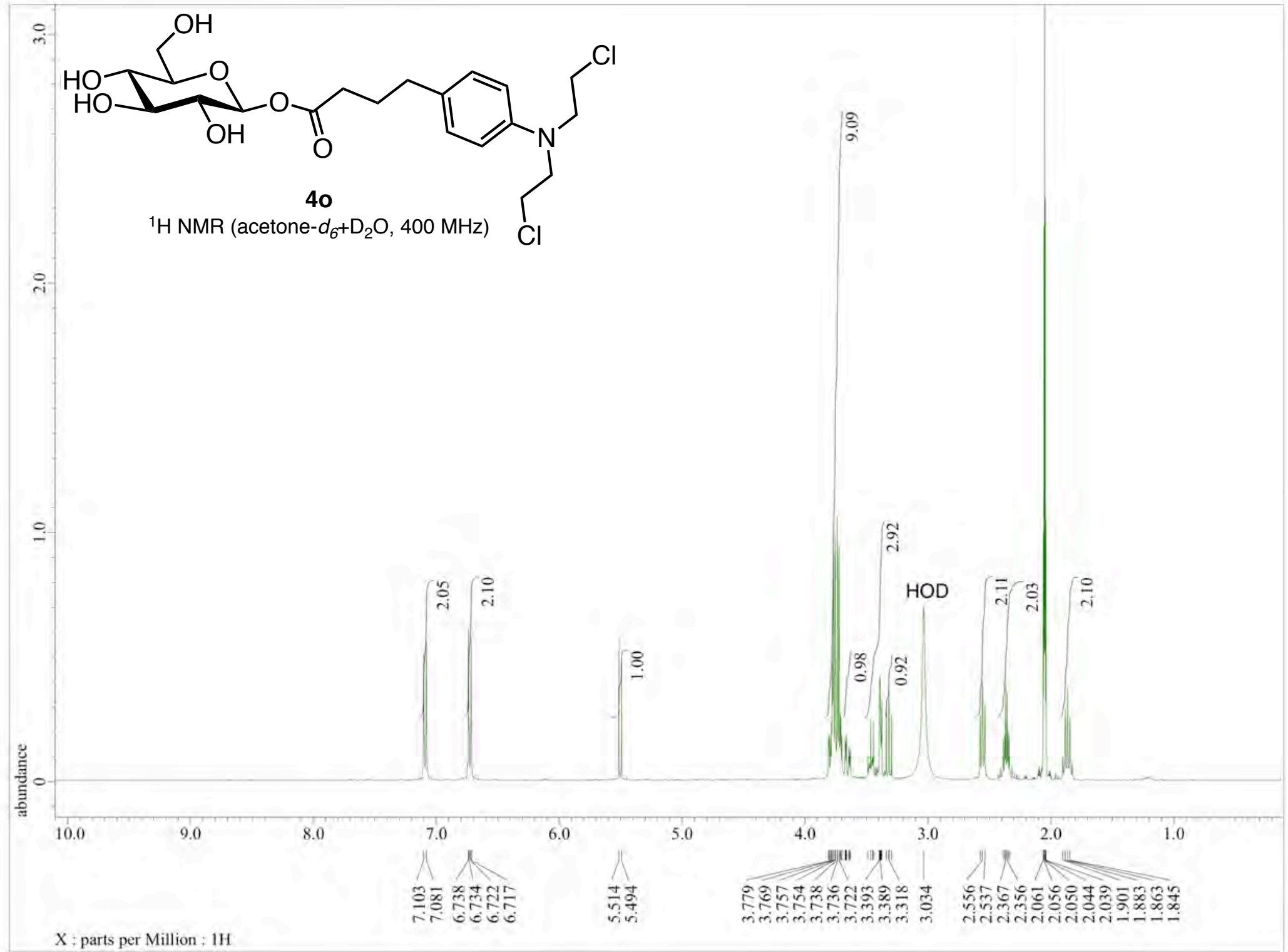




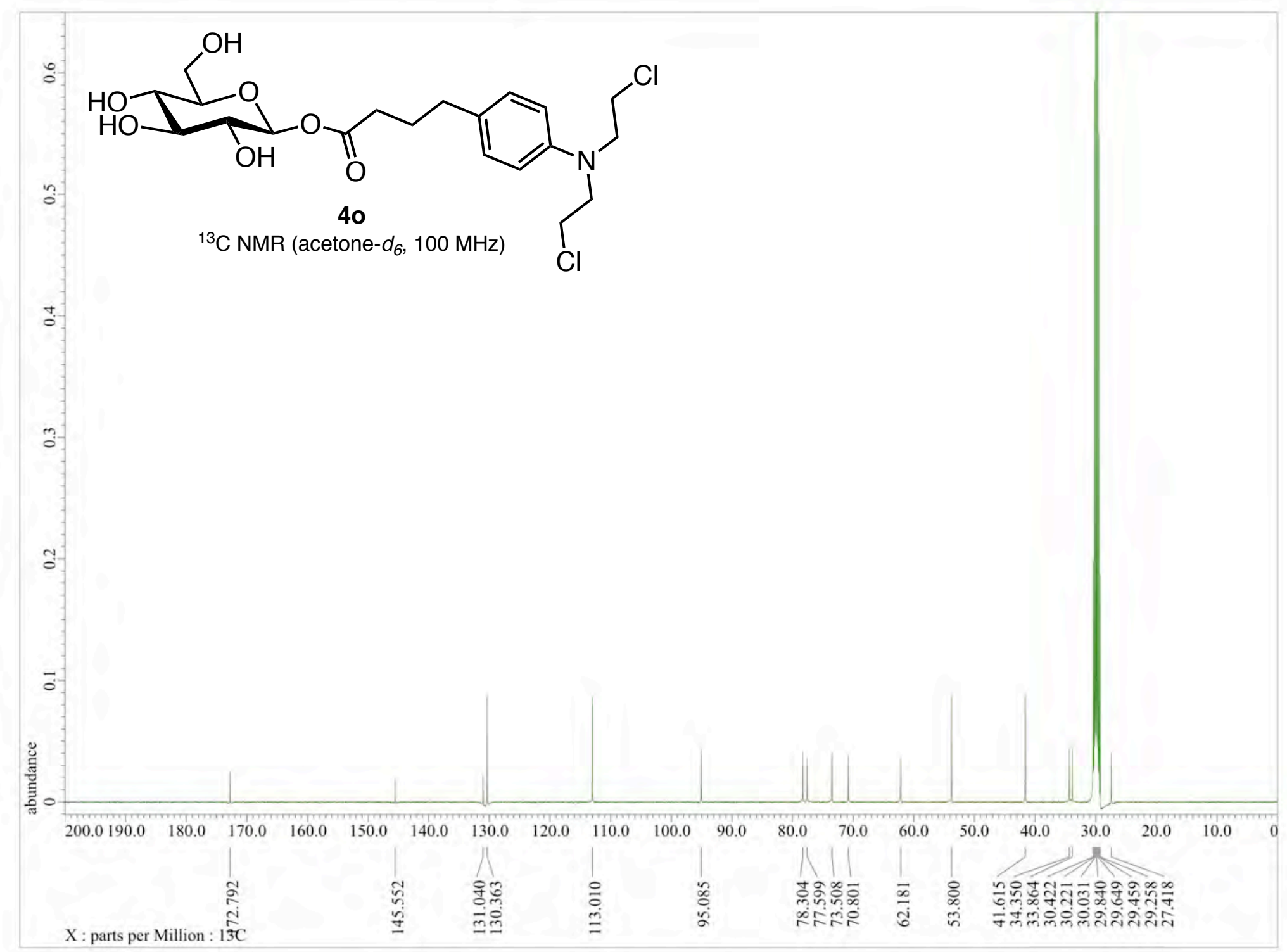




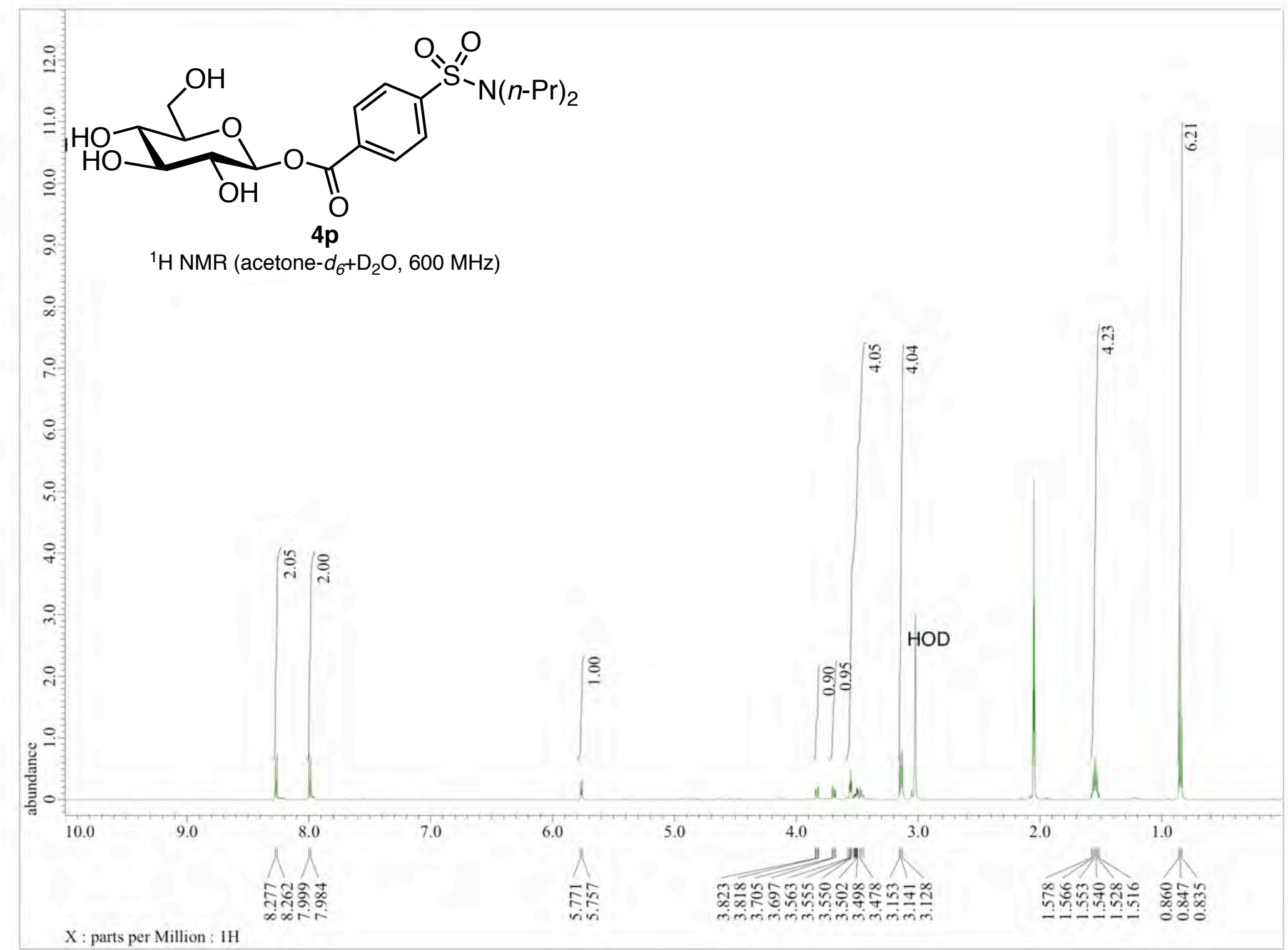




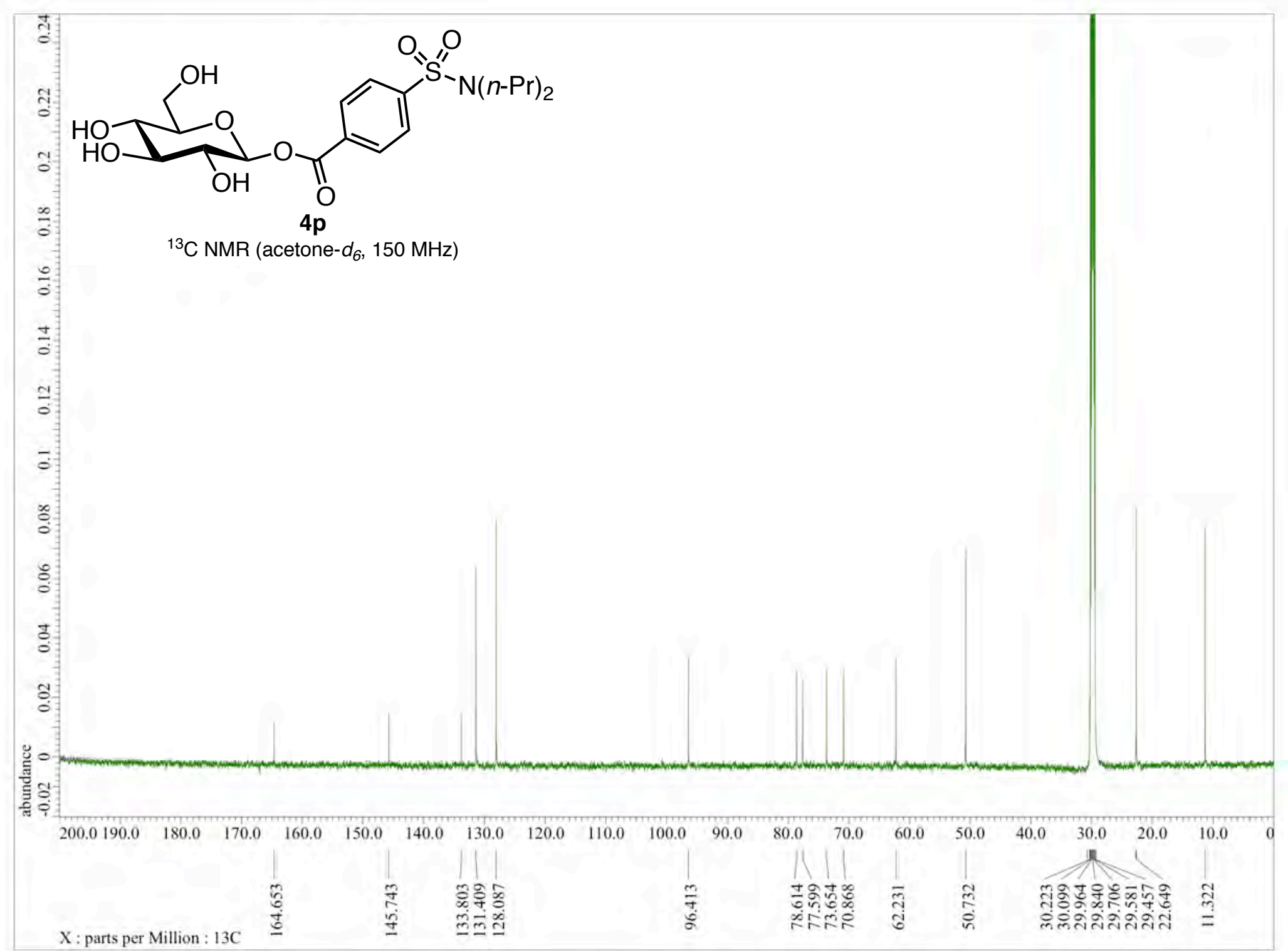




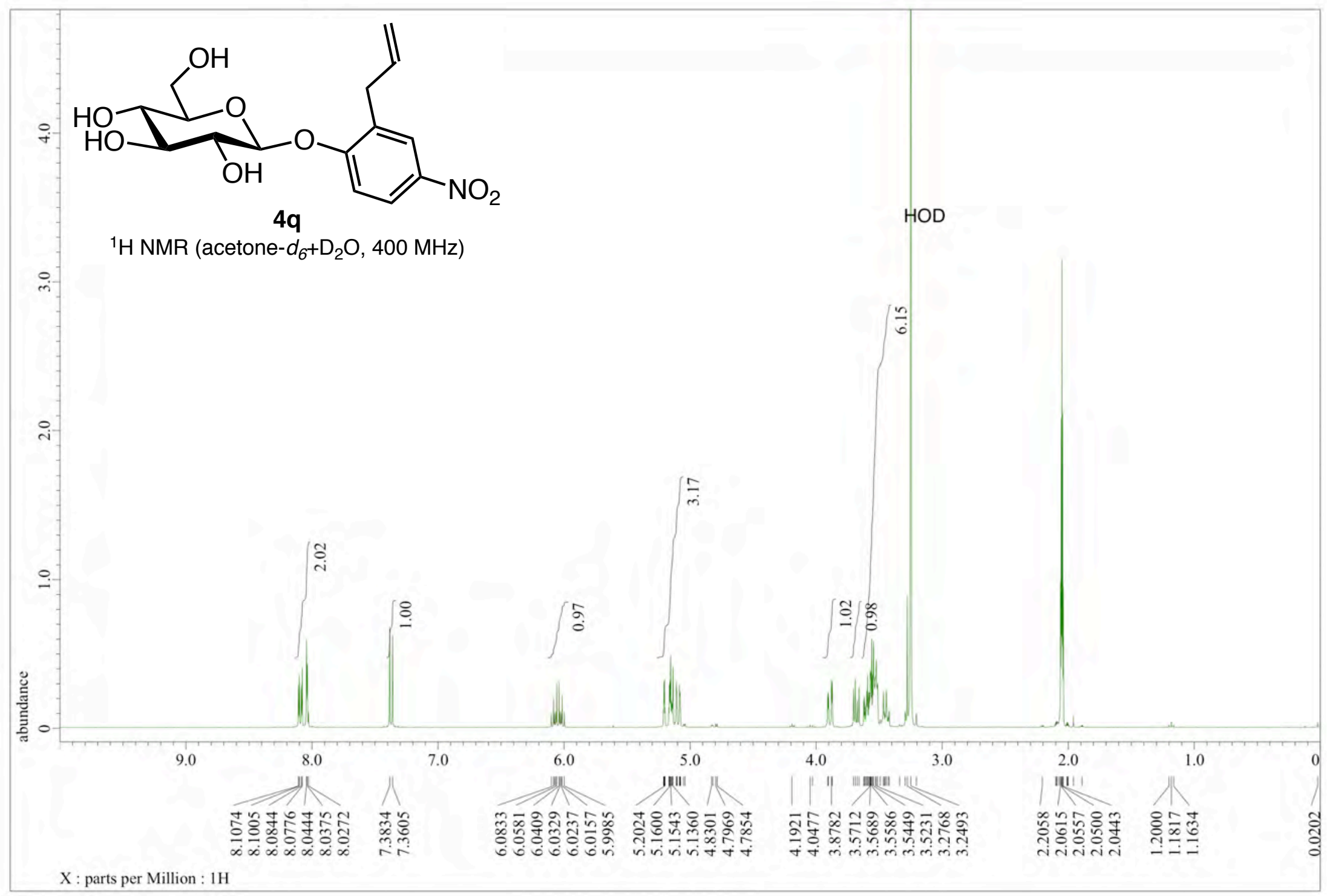




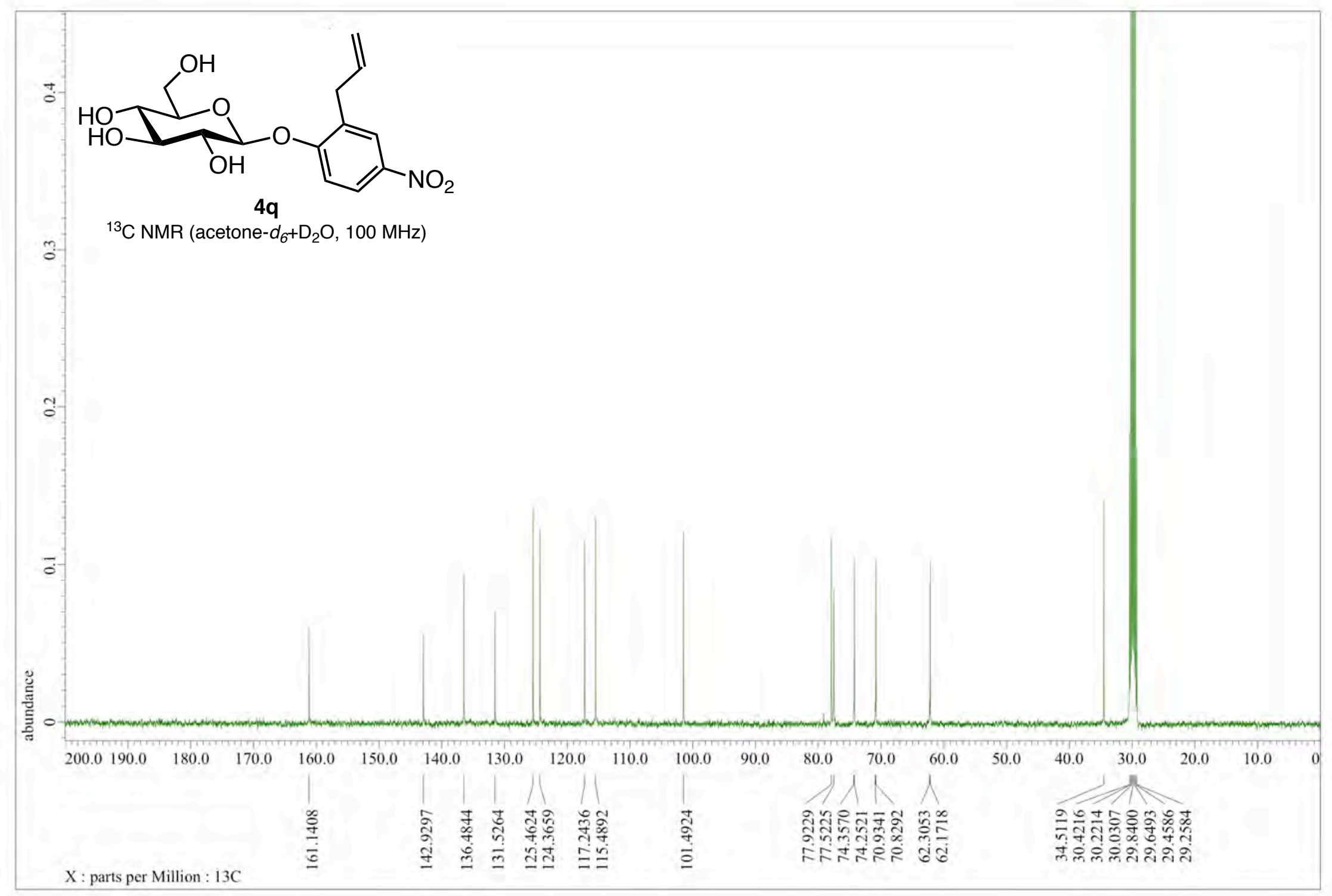




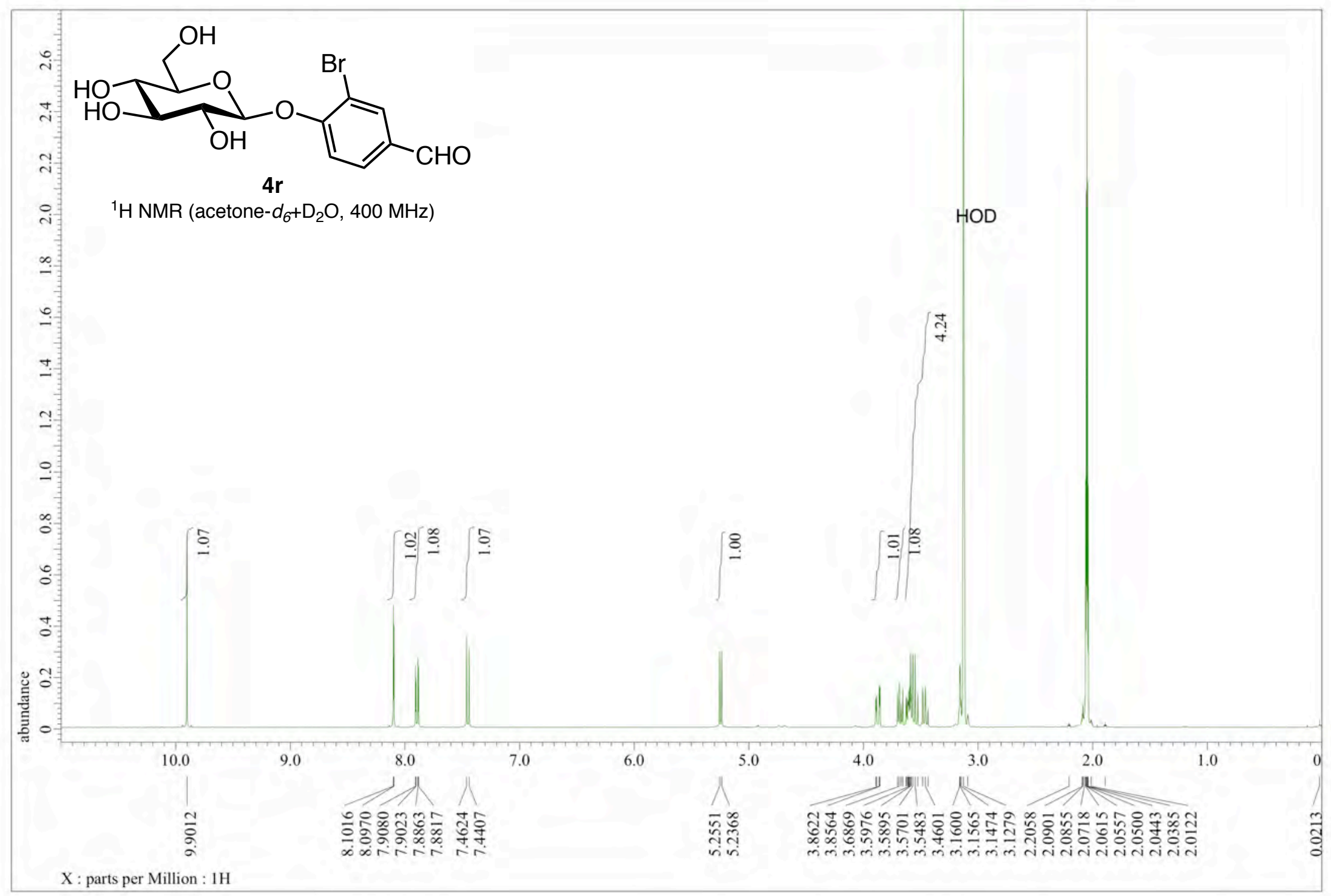




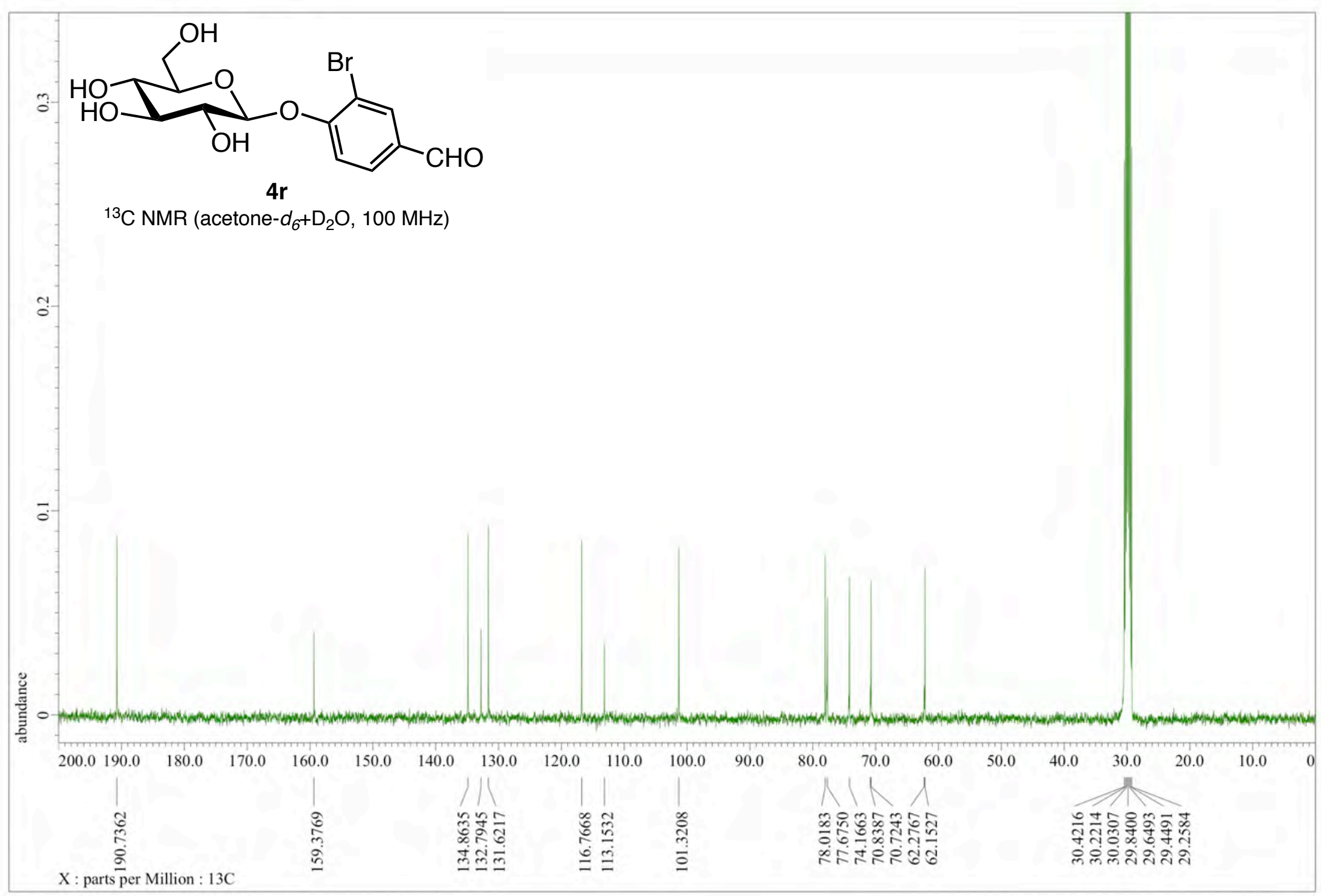




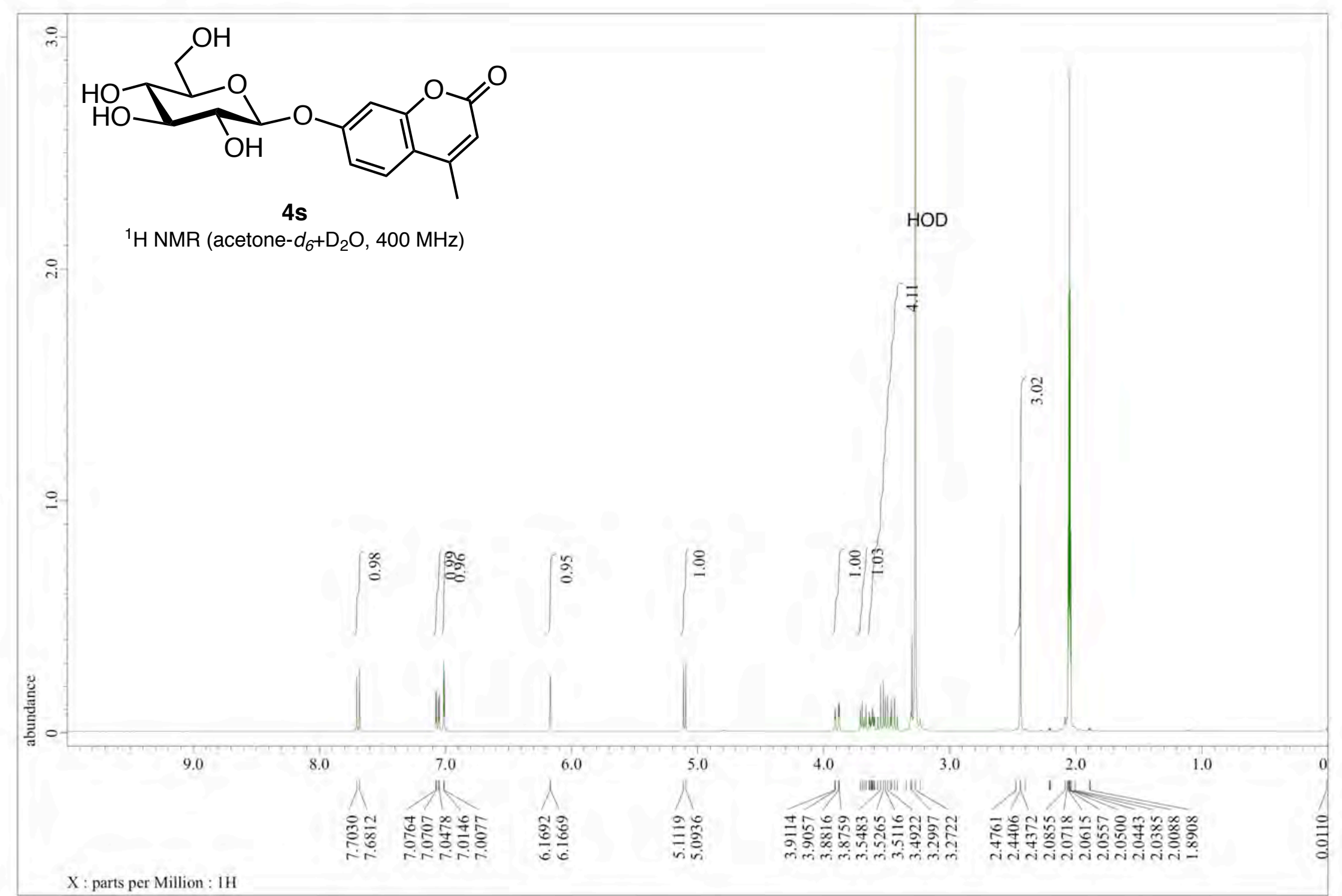




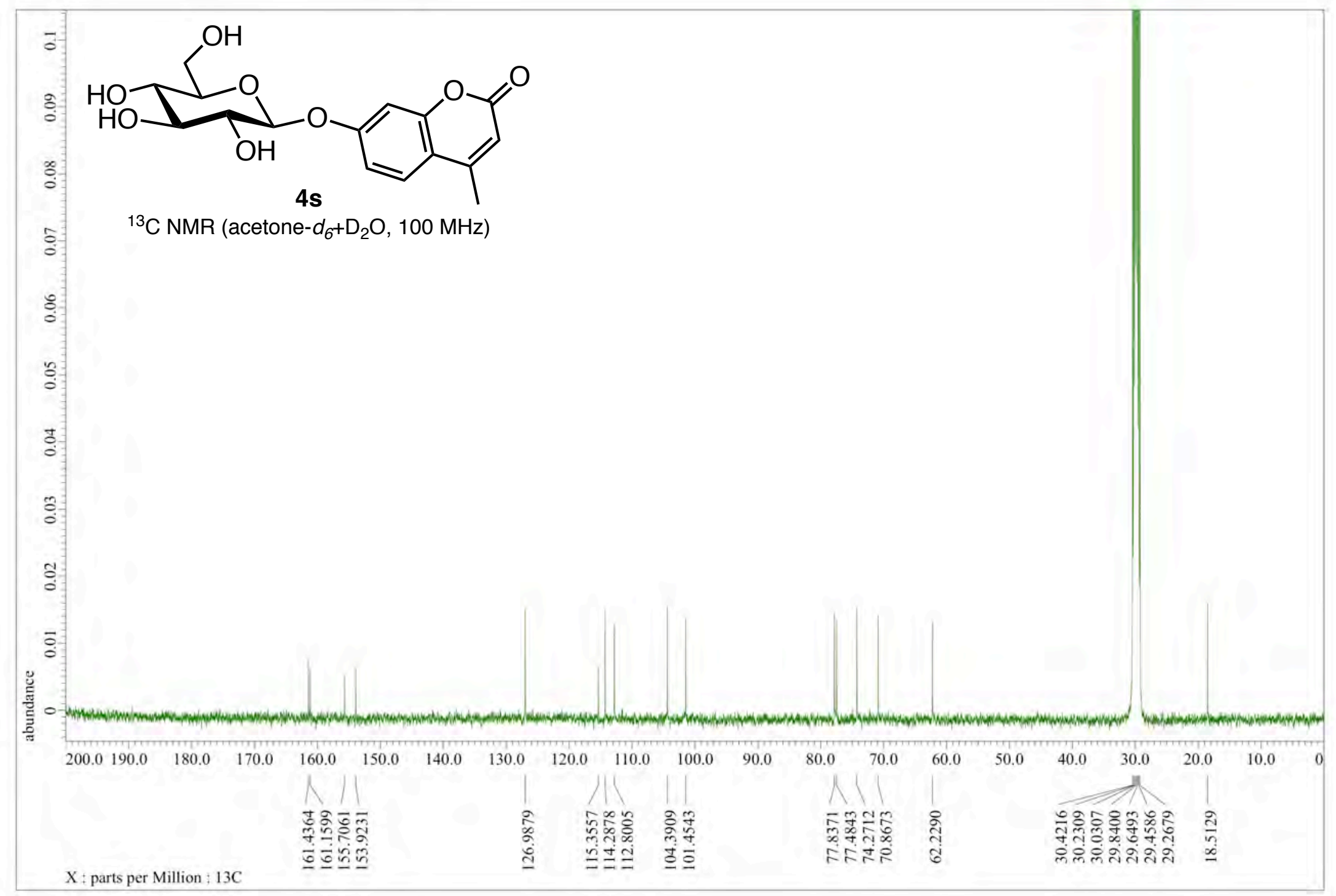




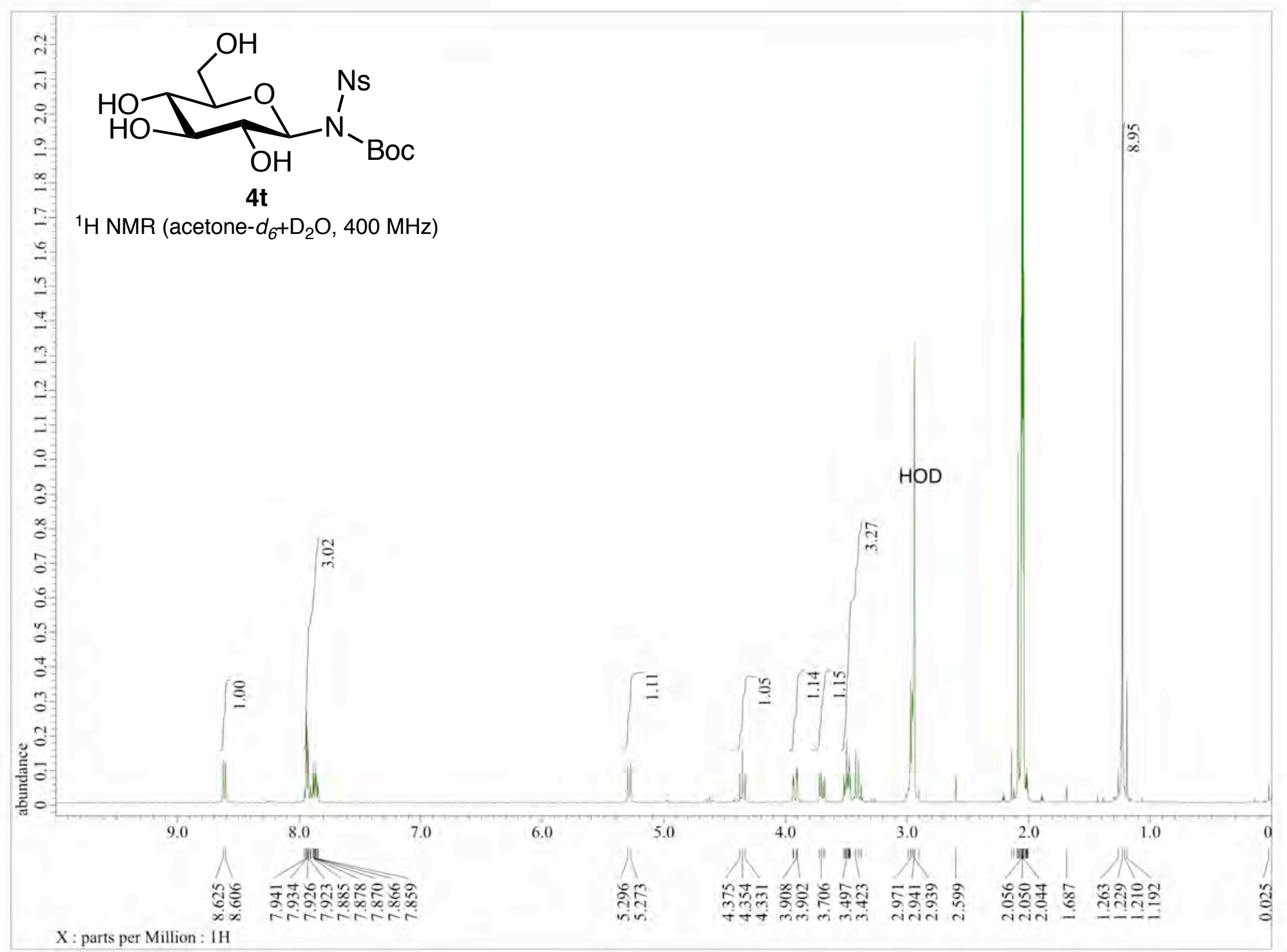




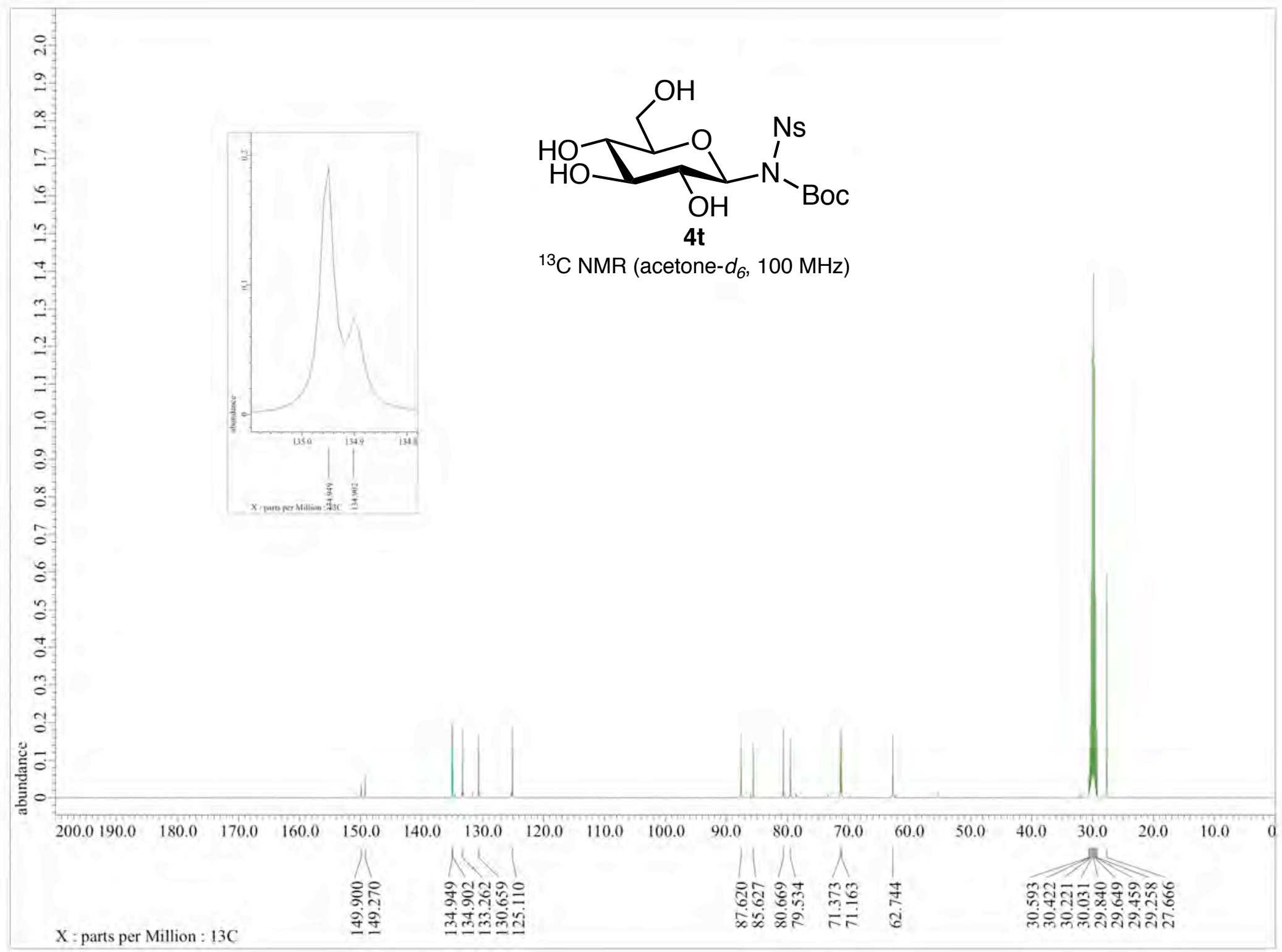




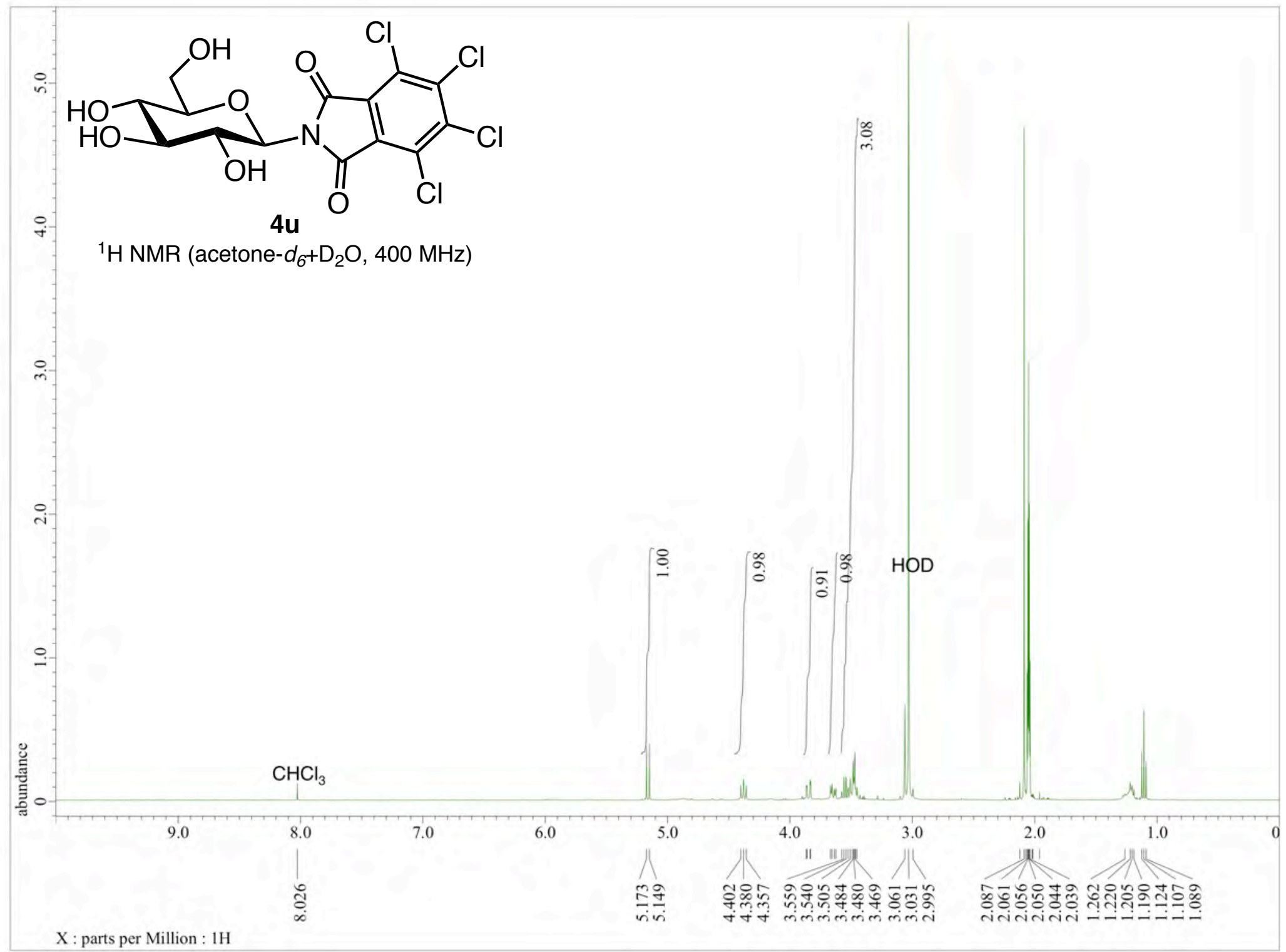




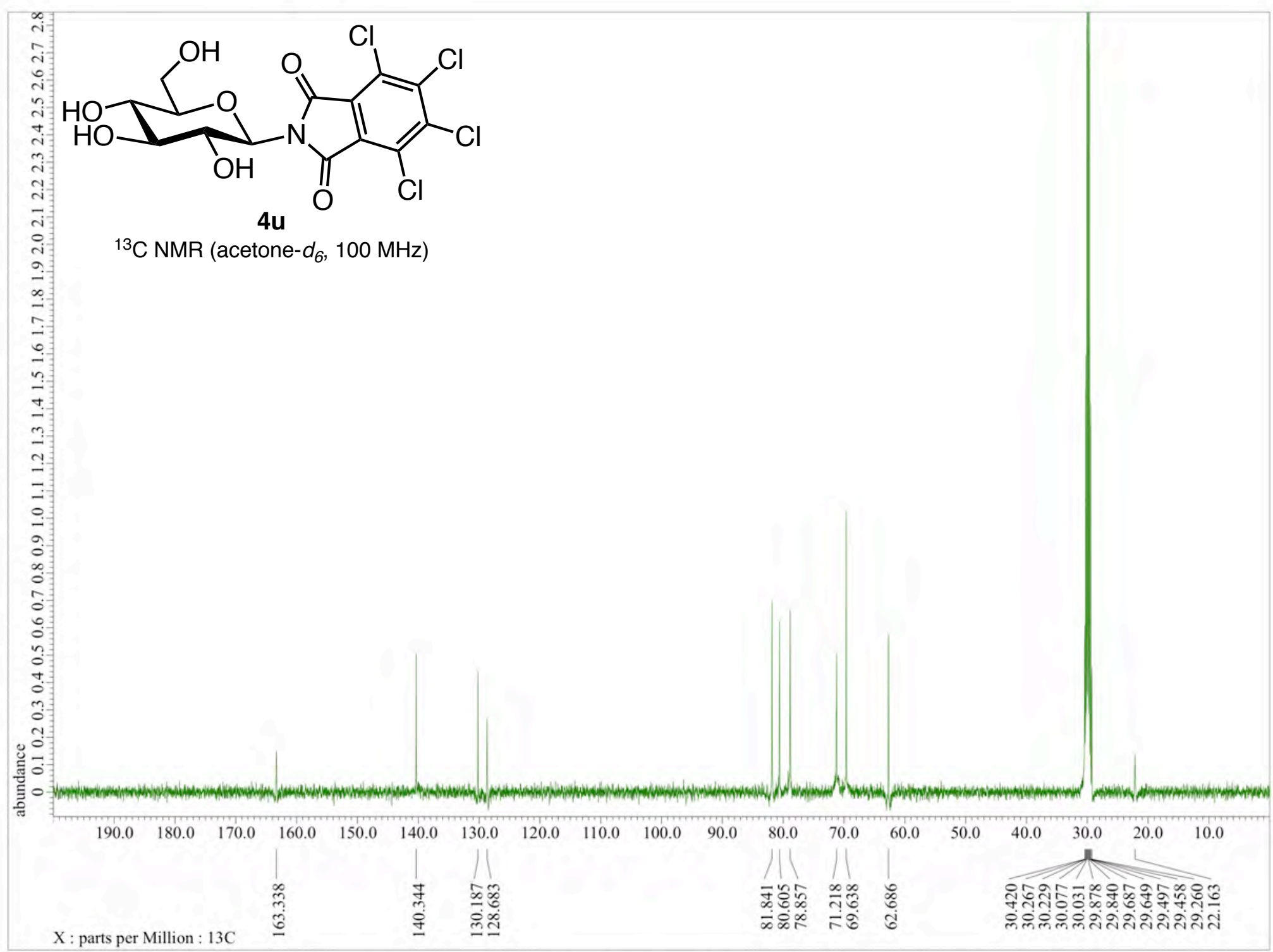




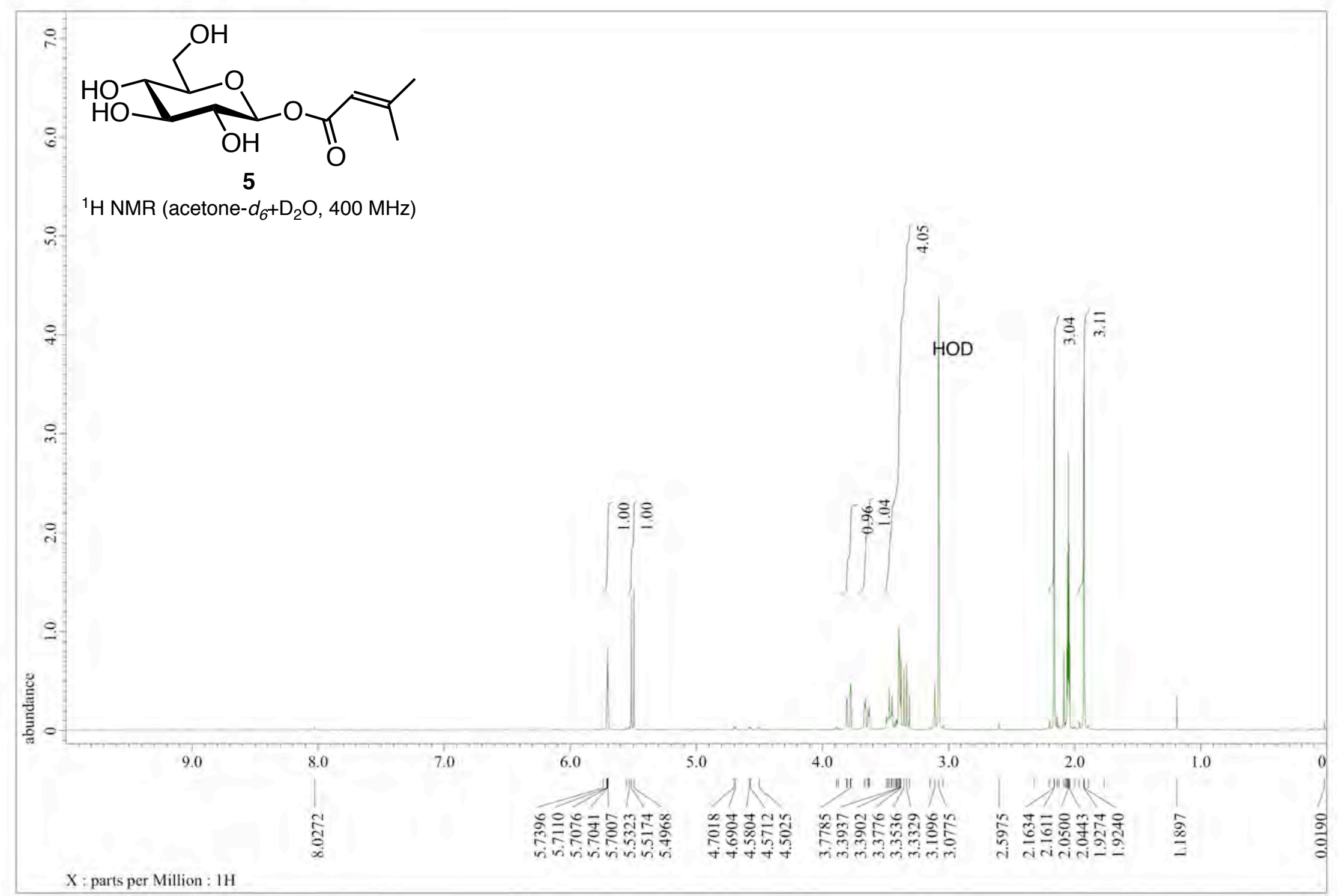




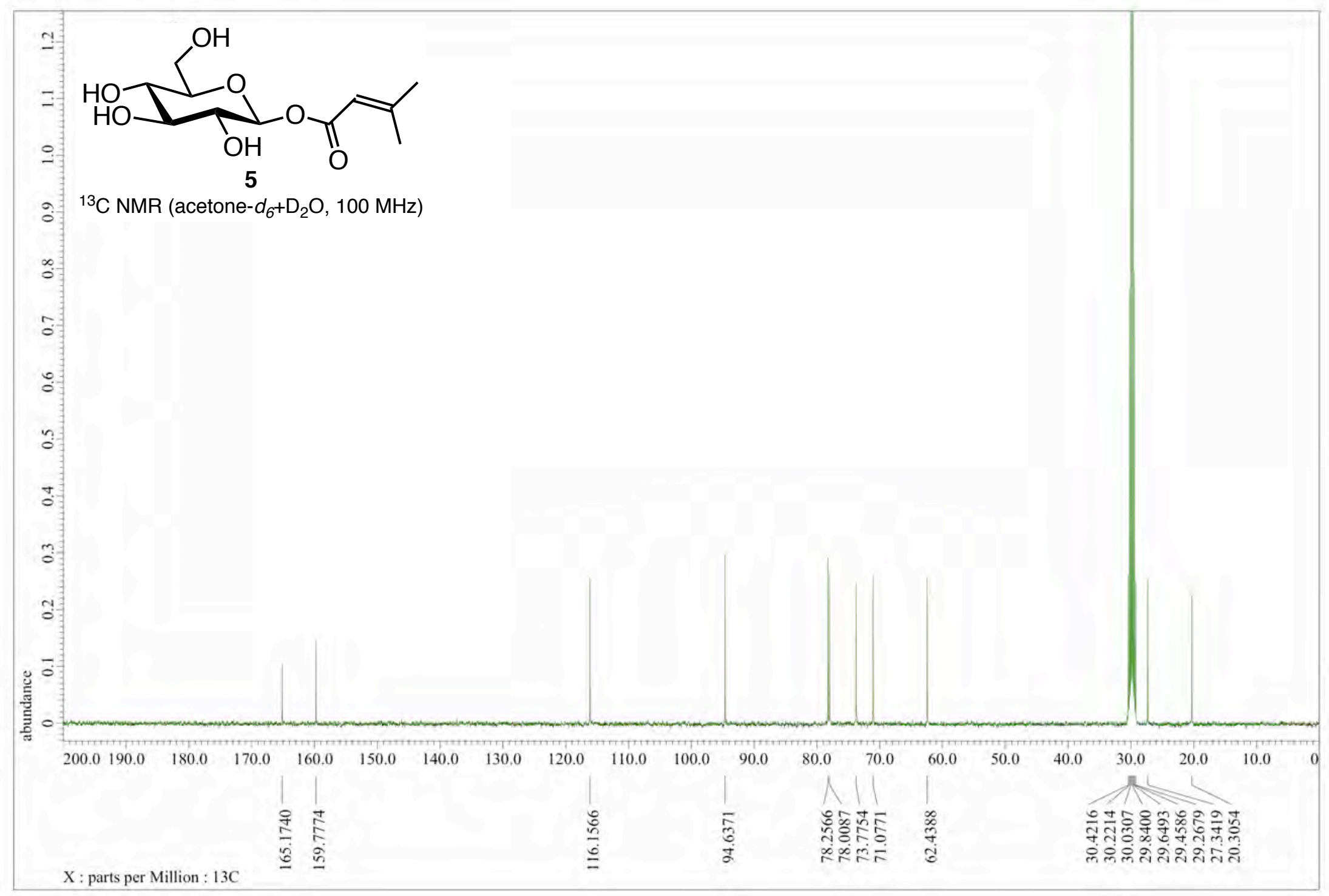




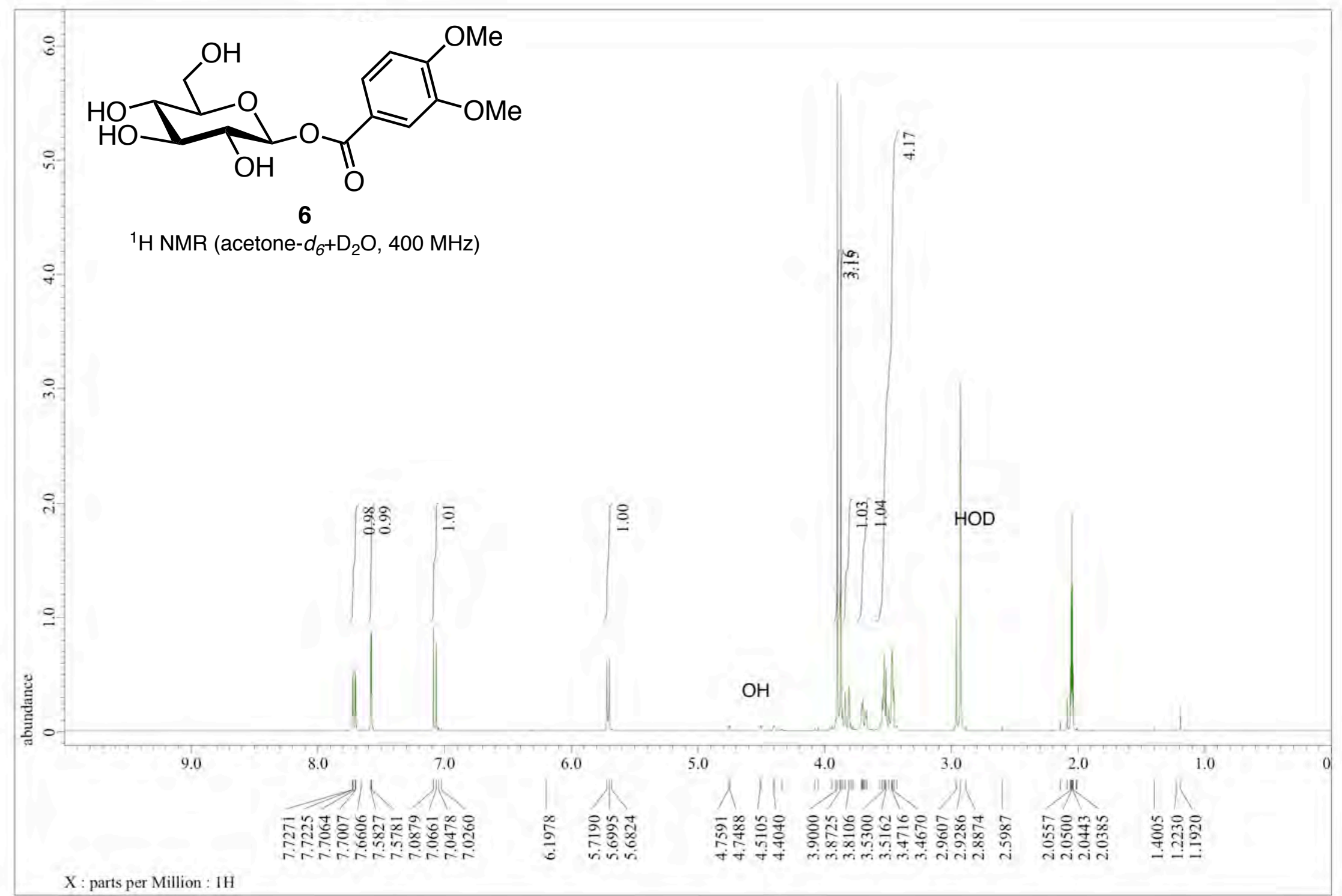




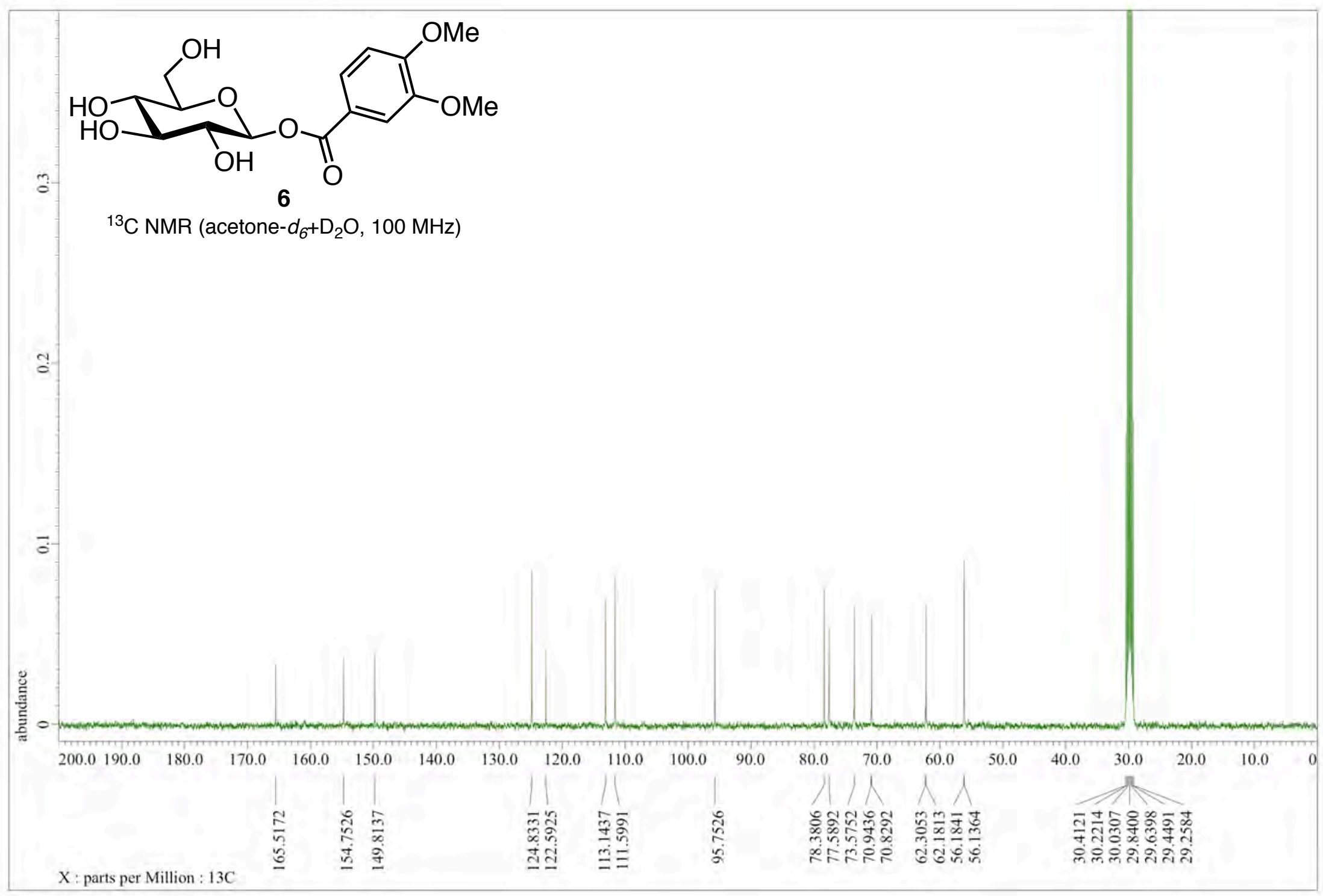




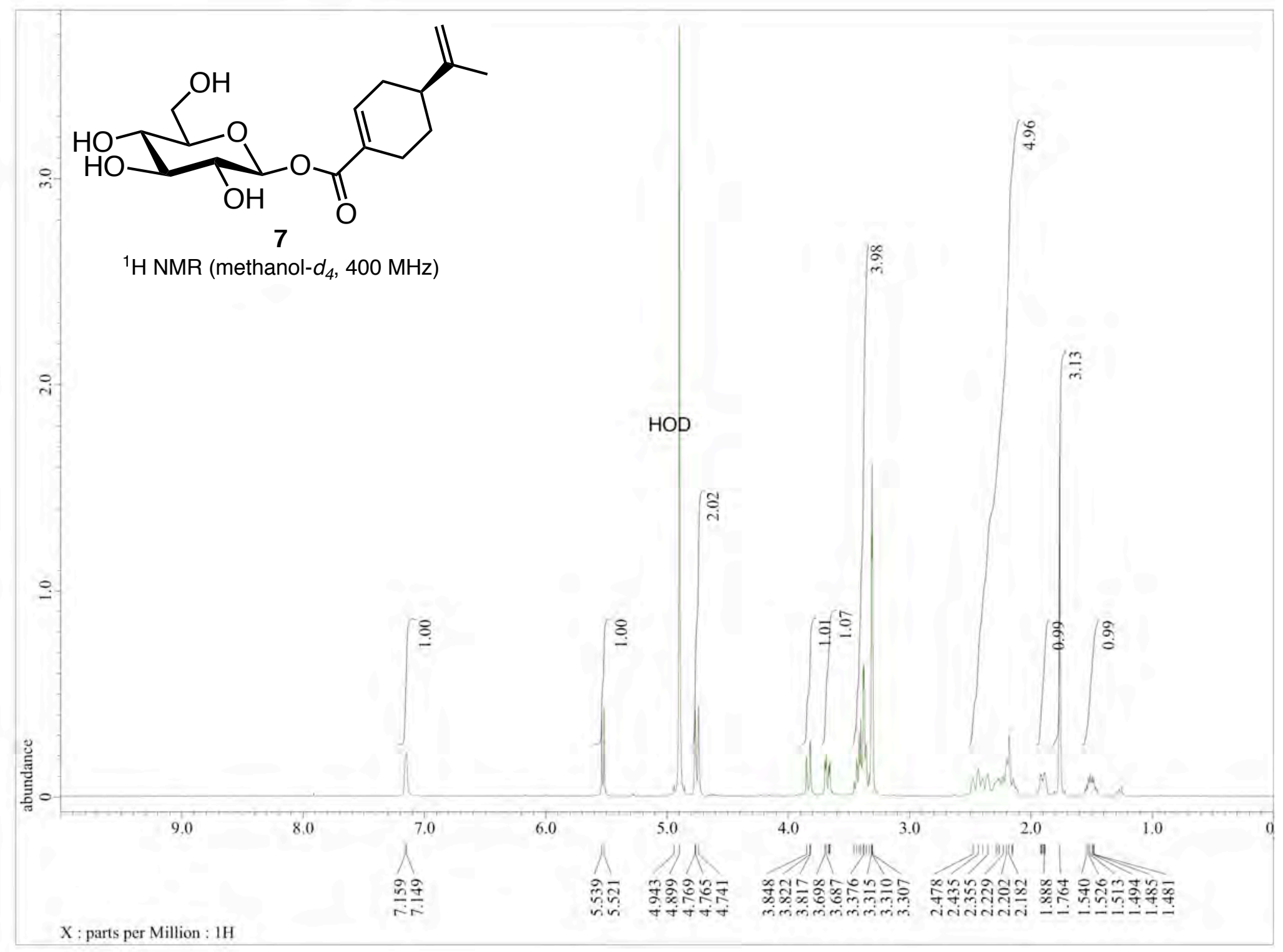




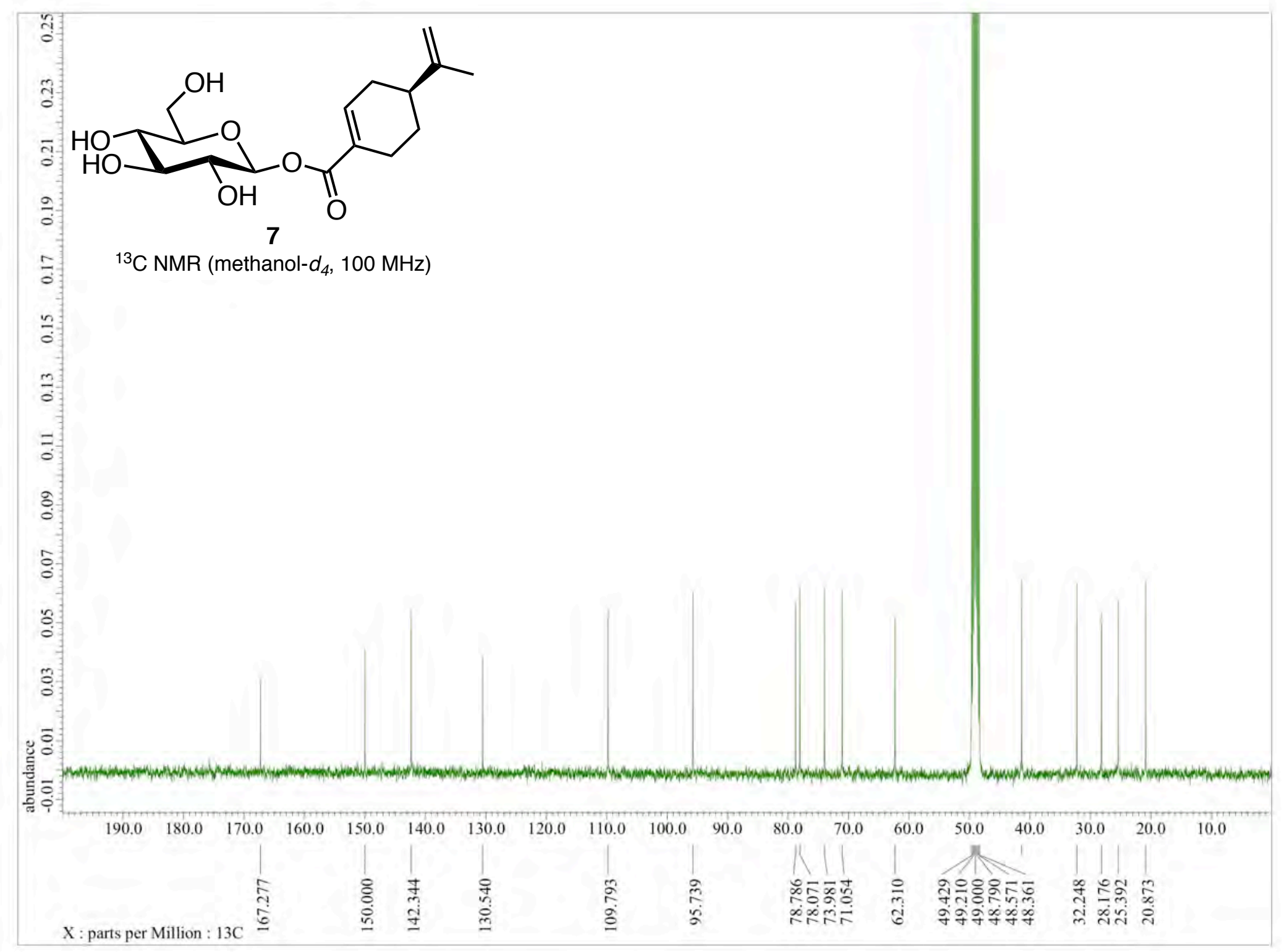




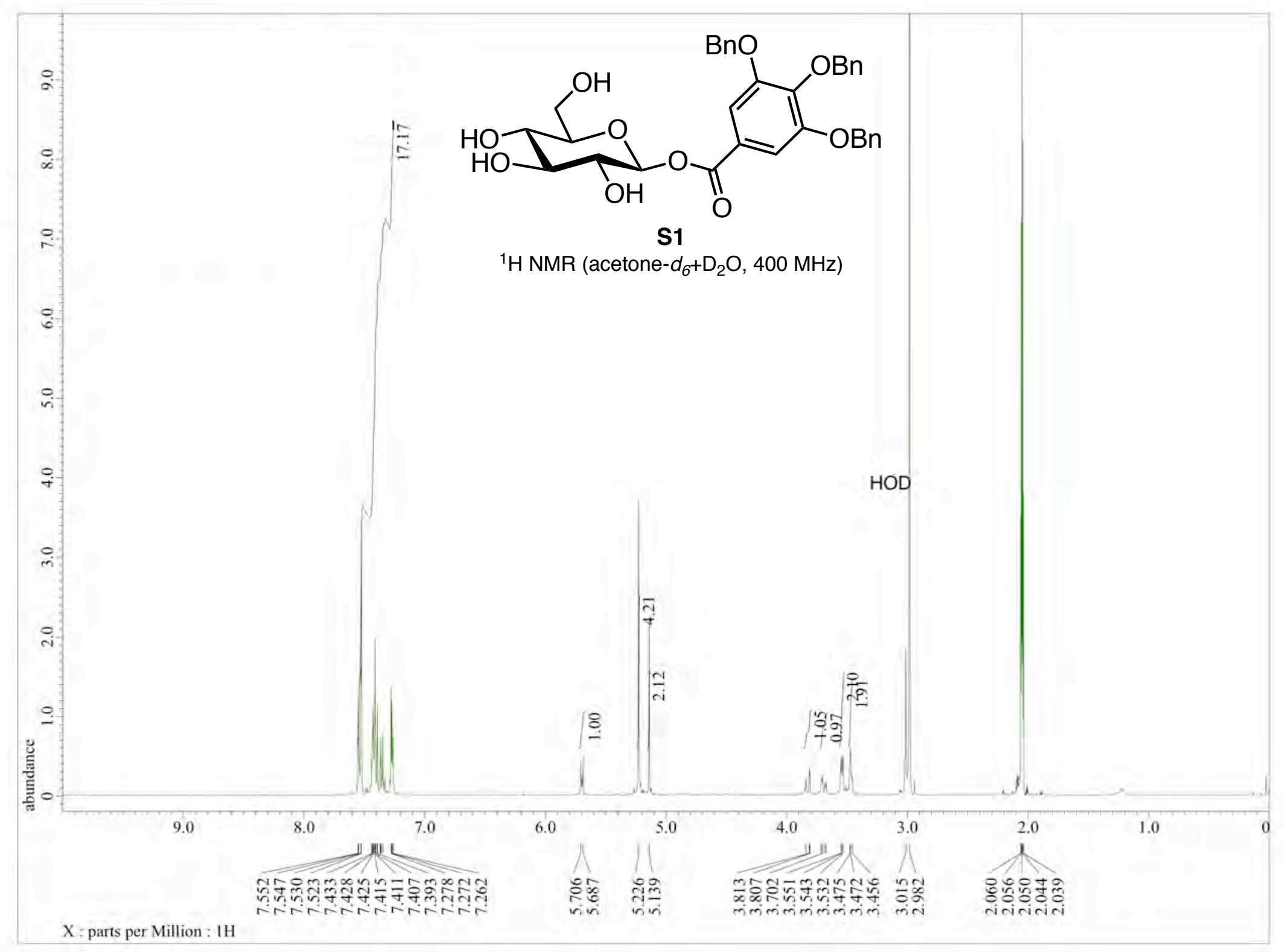




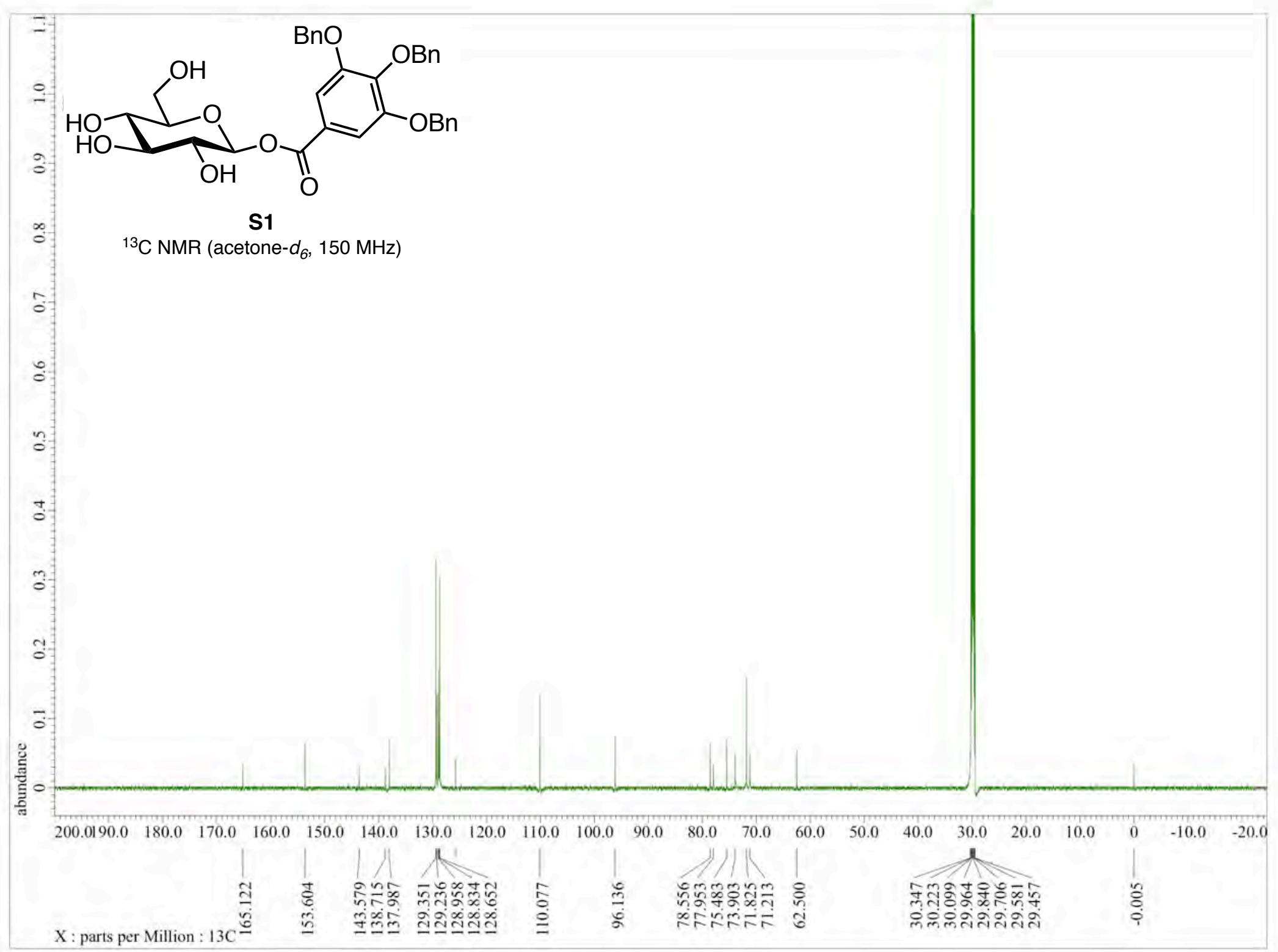




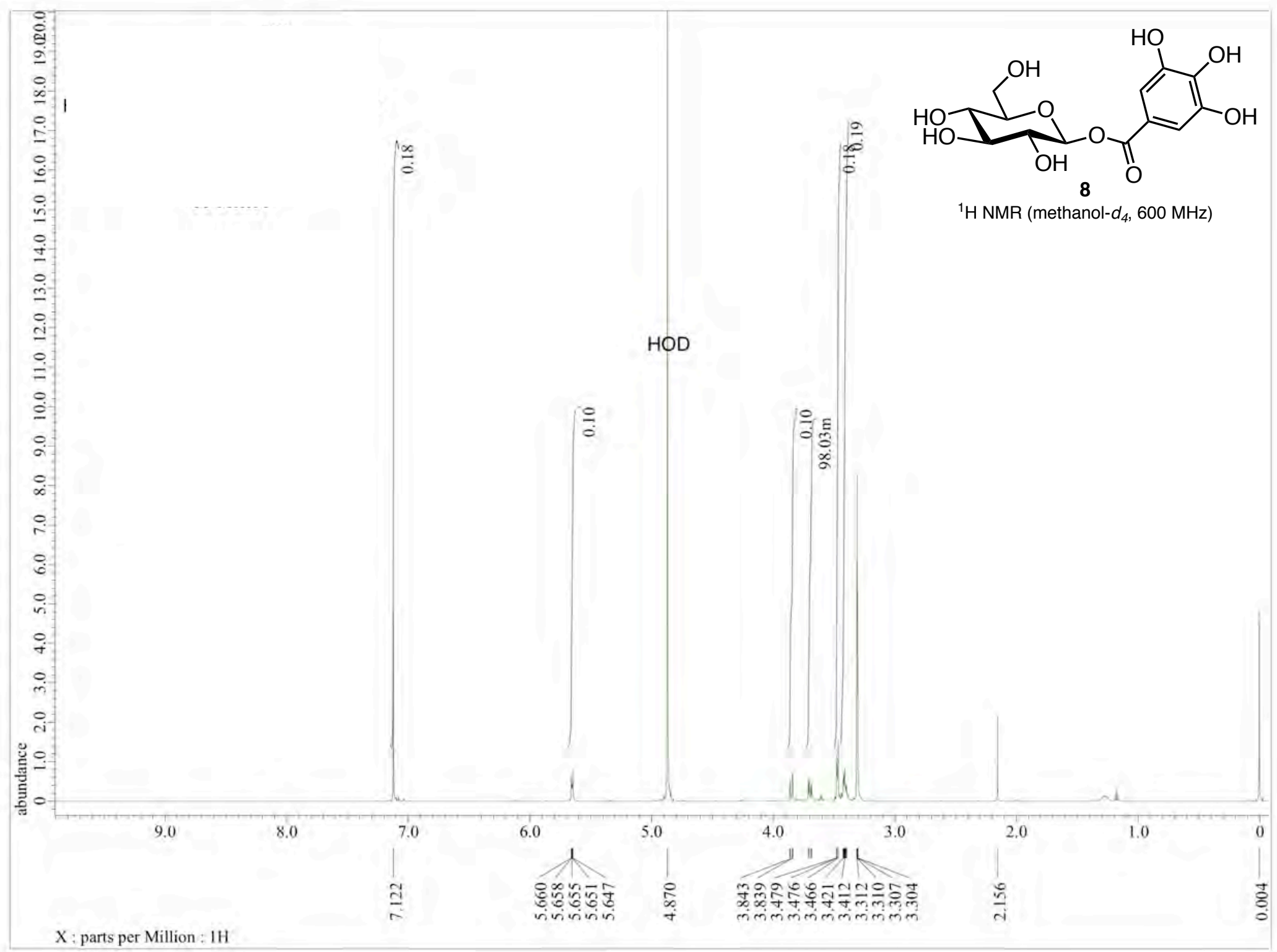




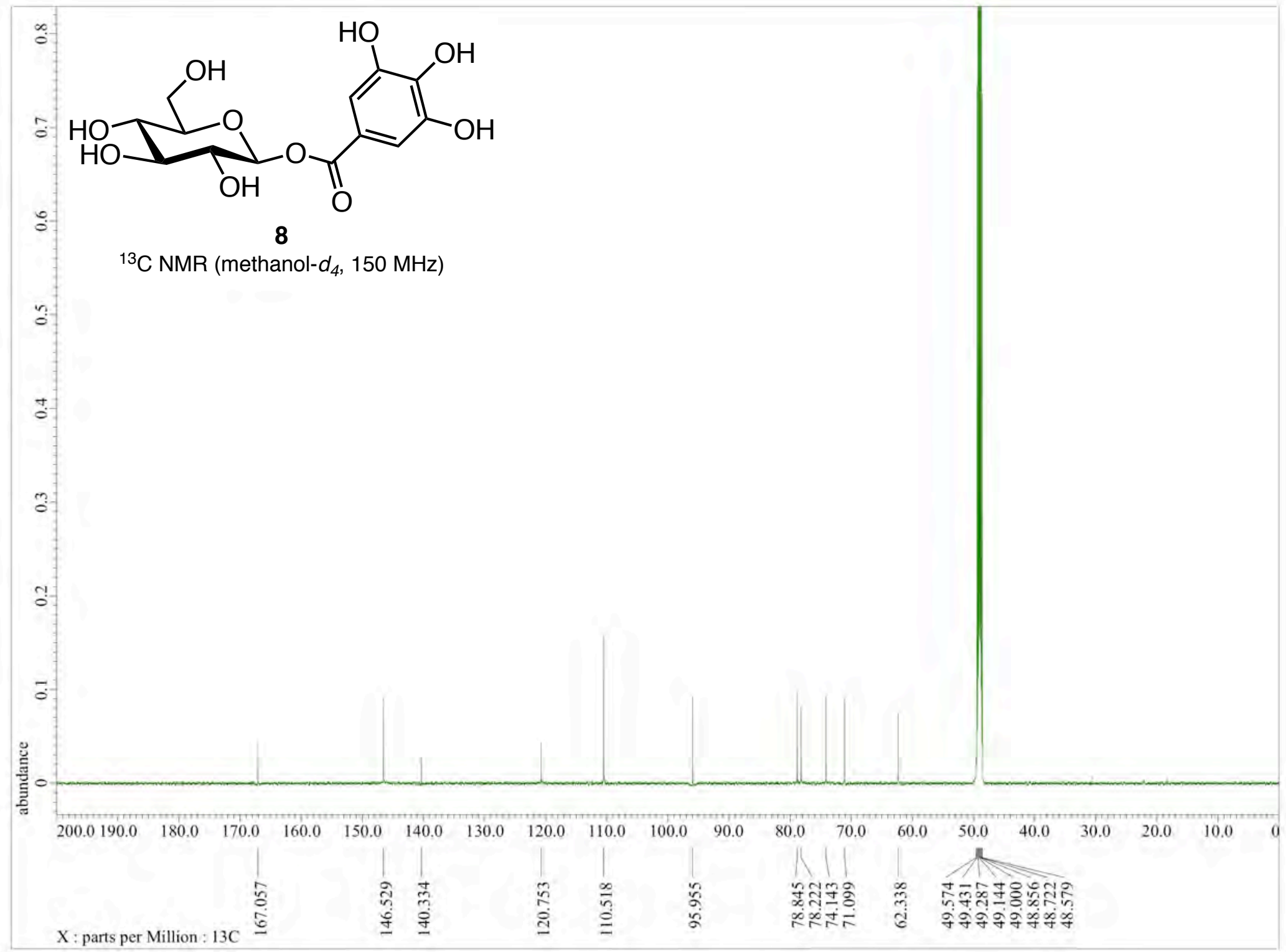




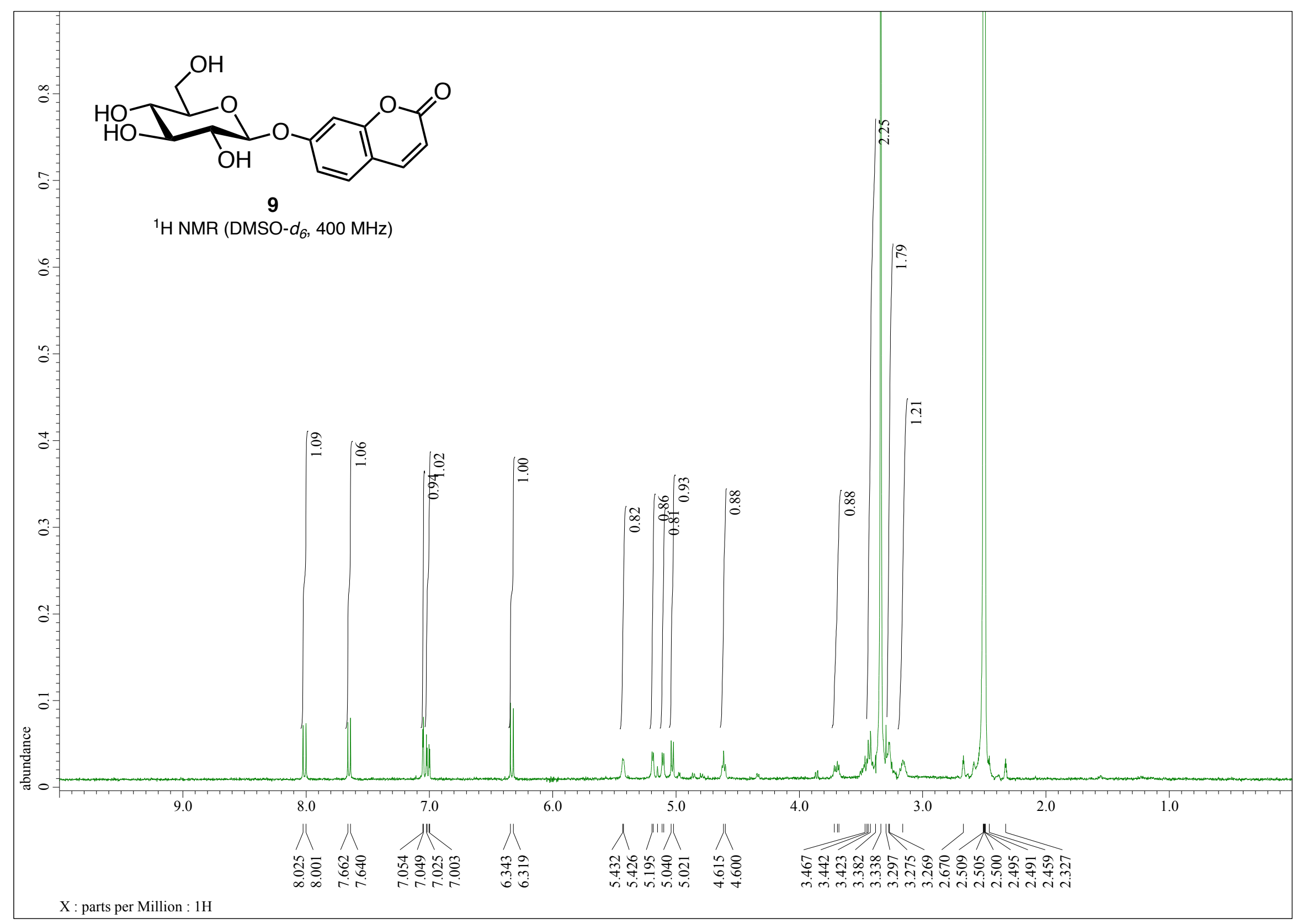




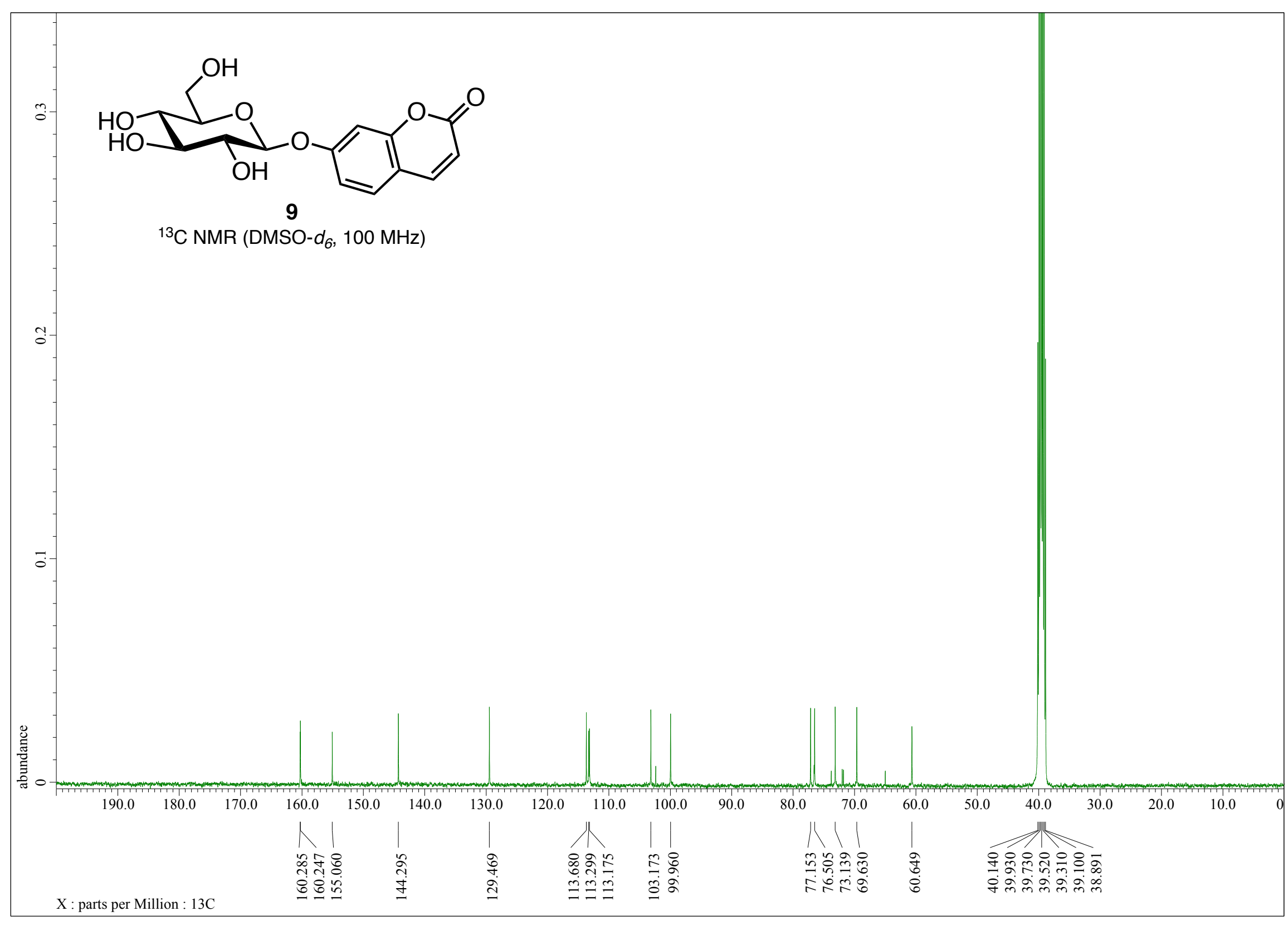

